\title{
The deubiquitinase USP32 regulates non-proteolytic ubiquitination in the endosomal-lysosomal system
}

\author{
Dissertation \\ zur Erlangung des Doktorgrades \\ der Naturwissenschaften
}

\author{
vorgelegt beim Fachbereich 14 \\ Biochemie, Chemie und Pharmazie \\ der Johann Wolfgang Goethe-Universität \\ in Frankfurt am Main
}

von

Alexandra Hertel

aus Nürnberg

Frankfurt am Main 2021

D30 
vom Fachbereich 14 der

Johann Wolfgang Goethe-Universität als Dissertation angenommen.

Dekan: $\quad$ Prof. Dr. Clemens Glaubitz

Gutachter: Prof. Dr. Martin Grininger

Dr. Anja Bremm

Datum der Disputation: 


\section{Table of contents}

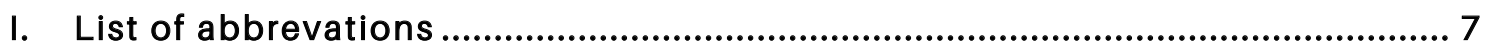

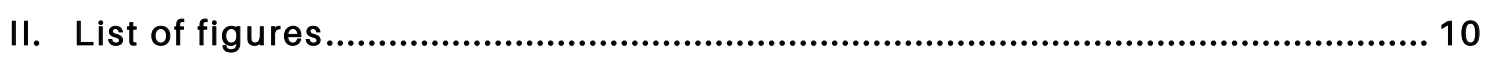

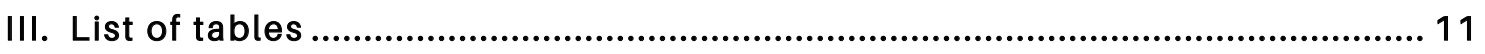

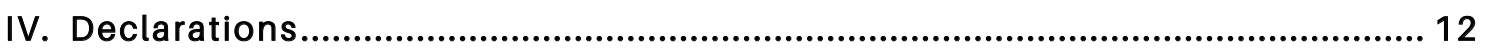

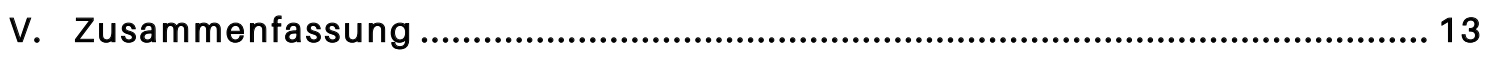

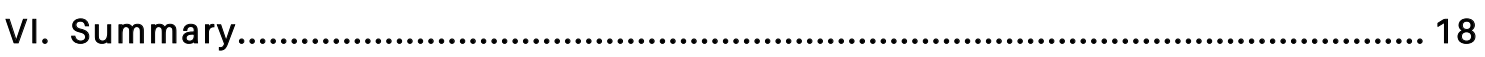

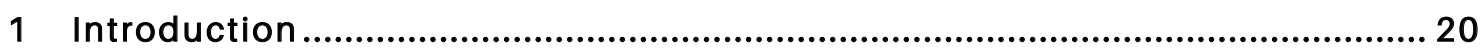

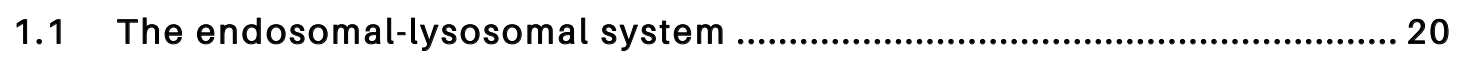

1.1.1 Early endosomes ...........................................................................................................20

1.1.2 Key regulators at the early endosome ......................................................................... 20

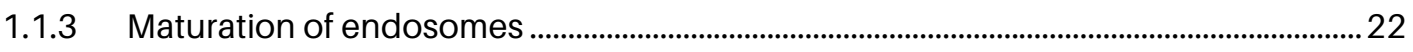

1.1.4 Late endosomes and endolysosomes.............................................................................. 23

1.1.5 Rab7 as key regulator of late endosomes and autophagy ...............................................2

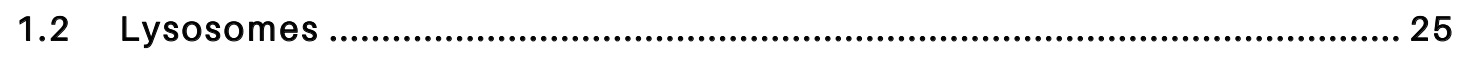

1.2.1 mTORC1 signaling at lysosomes........................................................................................26

1.2.2 Transcriptional regulation of mTORC1 via TFEB.............................................................2

1.2.3 Initiation of autophagy by mTORC 1 ................................................................................... 27

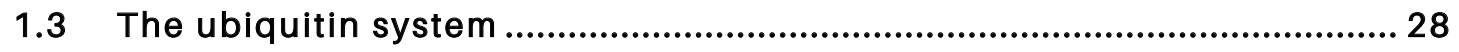

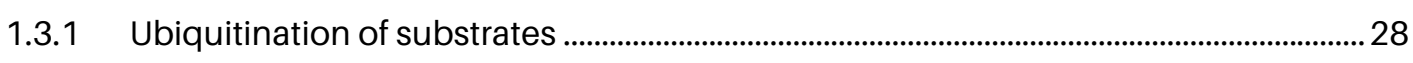

1.3.2 Deubiquitinating enzymes................................................................................................... 30

1.3.3 Ubiquitin regulation of the endosomal-lysosomal system .............................................32

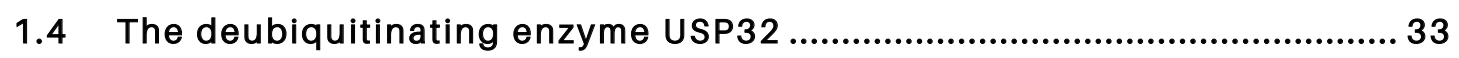

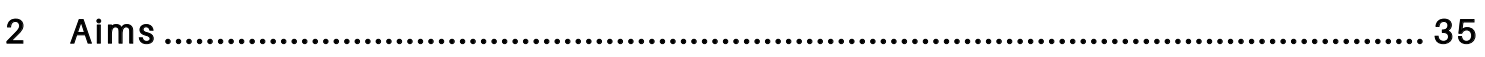

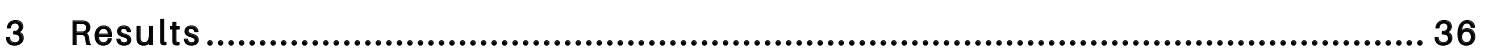

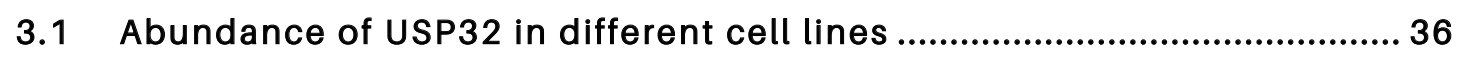

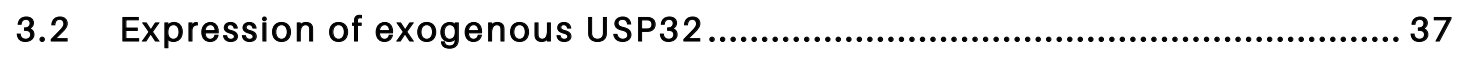

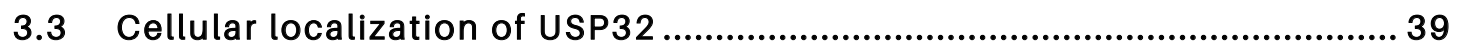

3.4 Identification of USP32 substrates (diGly IP) .......................................... 41

3.5 Characterization of Rab7 as USP32 substrate .......................................... 43

3.5.1 Interaction of USP32 and Rab7 .......................................................................................... 43

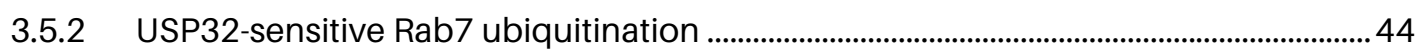

3.5.3 Rab7 localization at late endosomes and lysosomes ........................................................ 44

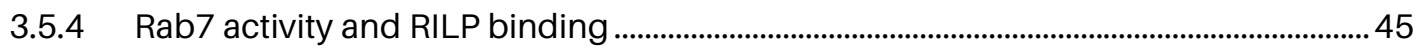

3.5.5 Rab7 mediated Cl-M6PR trafficking ............................................................................. 46

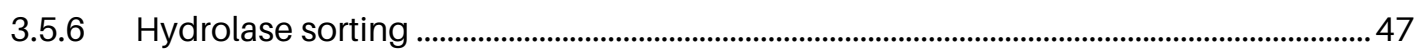

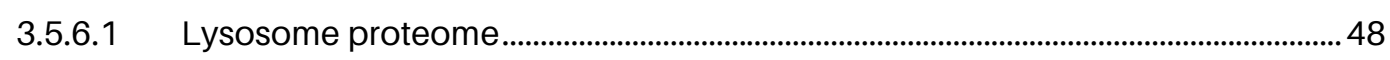

3.5.6.2 Lysosome content on immunoblot........................................................................ 48 
3.5.6.3 Hydrolase secretion ...............................................................................................

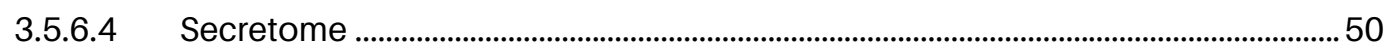

3.5.6.5 Secreted proteins on immunoblot ................................................................................ 52

3.5.6.6 Lysosomal degradation efficiency (DQ-BSA assay) ................................................52

3.5.7 USP32 effect on retromer complex......................................................................................53

3.5.7.1 CRC protein levels and composition ........................................................................53

3.5.7.2 USP32-CRC interaction ............................................................................................. 54

3.6 Characterization of LAMTOR1 as USP32 substrate ..................................55

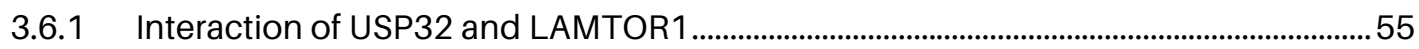

3.6.2 USP32-sensitive LAMTOR1 ubiquitination .........................................................................56

3.6.3 LAMTOR1 localization at lysosomes ..................................................................................58

3.6.4 Lysosome positioning ....................................................................................................... 59

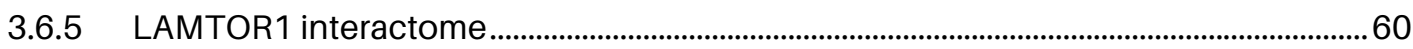

3.6.6 Effect on mTORC1 1..............................................................................................................62

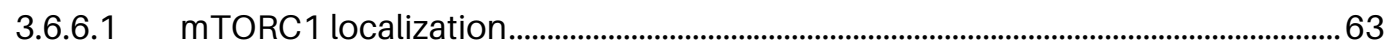

3.6.6.2 mTORC1 activity ......................................................................................................6

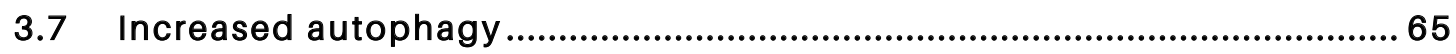

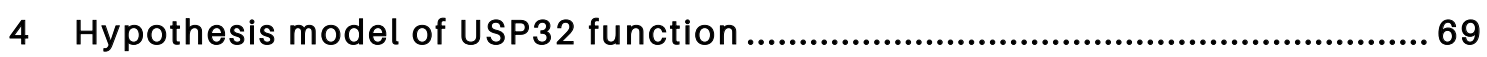

4.1 USP32 regulates non-proteolytic Rab7 ubiquitination and protein trafficking in the endosomal-lysosomal system ..............................................69

4.2 USP32 regulates non-proteolytic LAMTOR1 ubiquitination and Ragulator

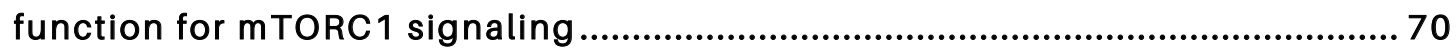

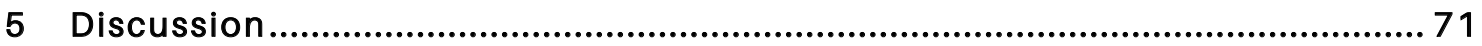

5.1 Establishing tools for the characterization of USP32 function without

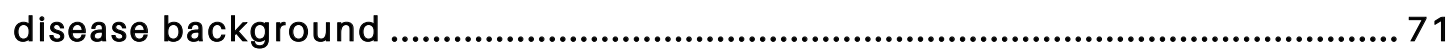

5.2 Identification of USP32 substrates in the endosomal-lysosomal system 72

5.3 Possible ubiquitin regulation of palmitoylation ....................................... 72

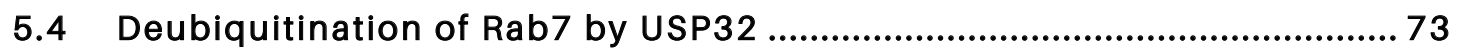

5.4.1 Effect of Rab7 ubiquitination on trafficking ...................................................................... 74

5.4.2 Competing data on Rab7 regulation through USP32 deubiquitination ......................75

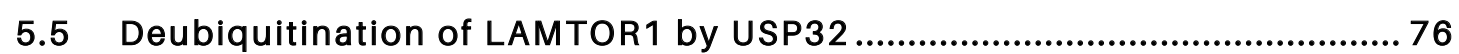

5.5.1 Effect of USP32-sensitive ubiquitination on LAMTOR1 ......................................................

5.5.2 USP32-dependent LAMTOR1 interactome studies........................................................... 78

5.5.3 Effect of USP32-sensitive ubiquitination of LAMTOR1 on mTORC1 ……………….......79

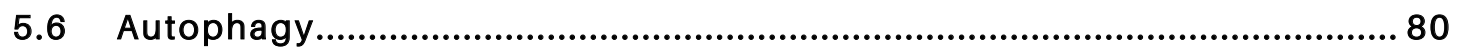

5.7 Rab11 and TMEM192 as USP32 substrates................................................ 81

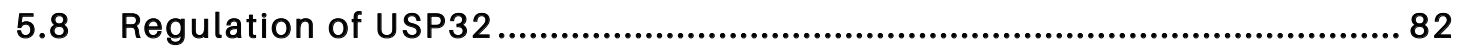

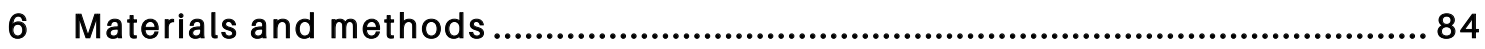

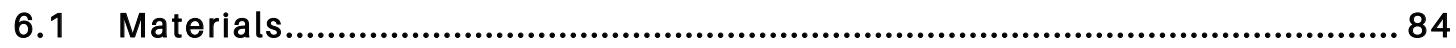




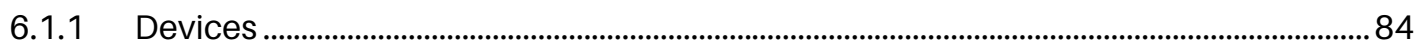

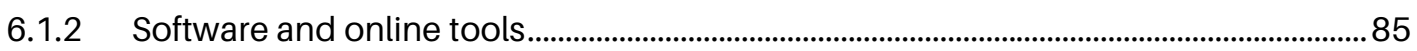

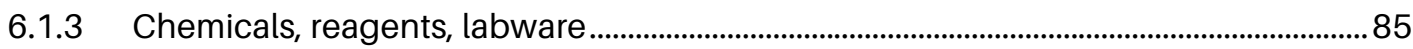

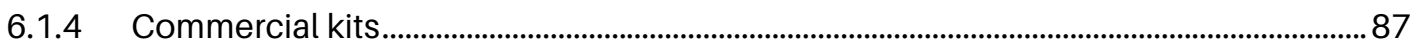

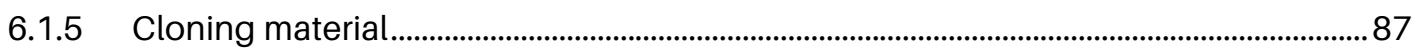

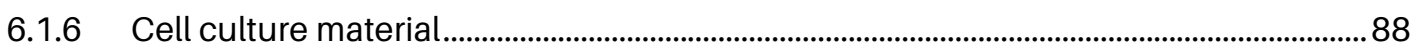

6.1.7 Mammalian cell lines and bacteria...........................................................................................8

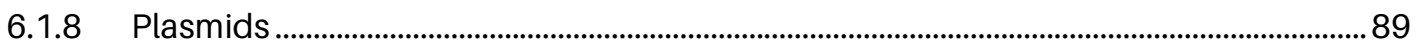

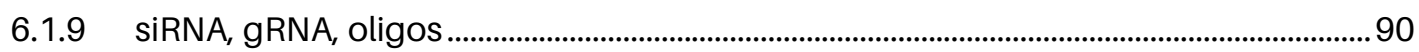

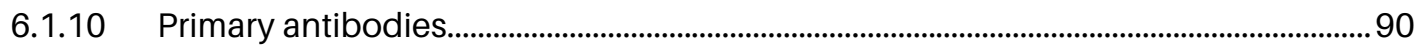

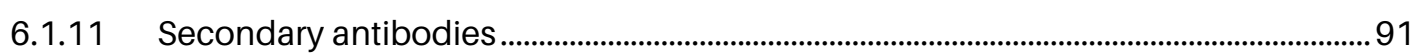

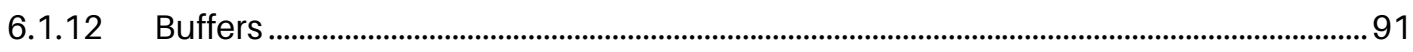

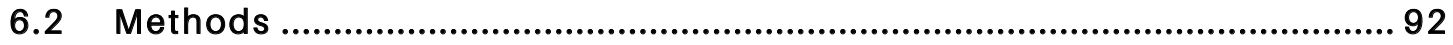

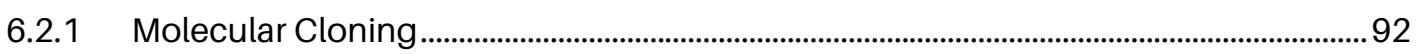

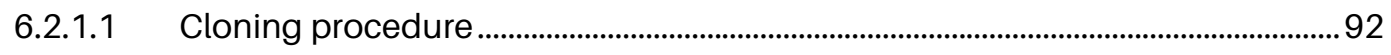

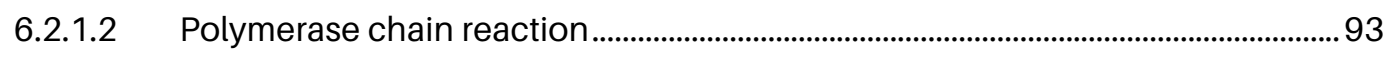

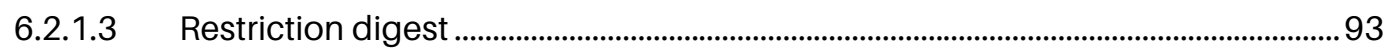

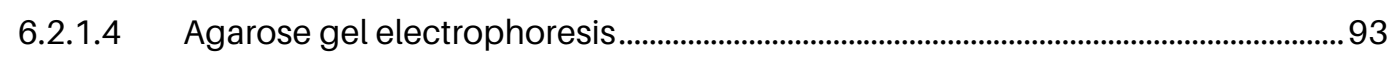

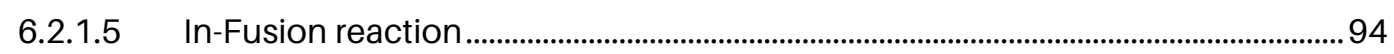

6.2.1.6 Transformation, amplification, purification and sequence verification .............94

6.2.1.7 Guide RNA design and CRISPR/Cas9 plasmid generation ......................................94

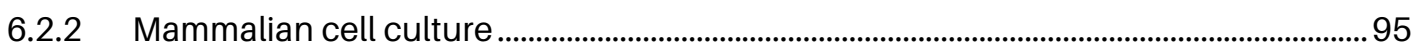

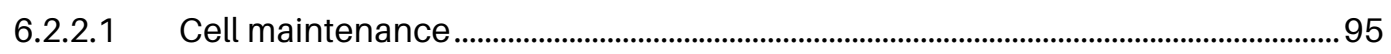

6.2.2.2 Cryopreserving and thawing cells ......................................................................................95

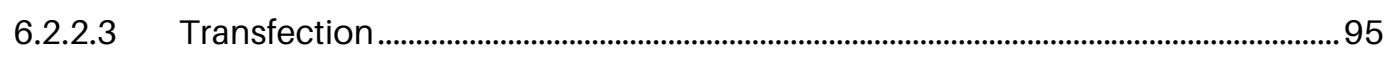

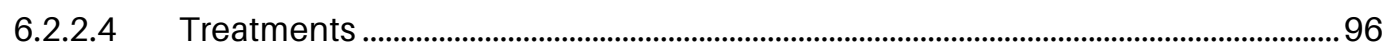

6.2.2.5 Generation of high-titer lentivirus and transduction of cells ..................................96

6.2.3 Immunofluorescence microscopy .................................................................................................96

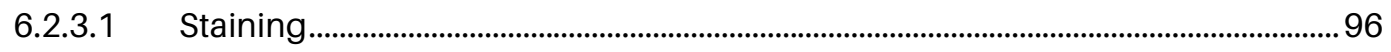

6.2.3.2 Cl-M6PR antibody-feeding assay .....................................................................................96

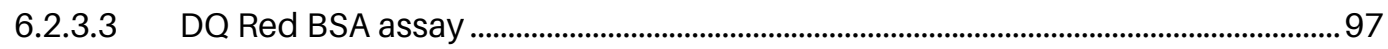

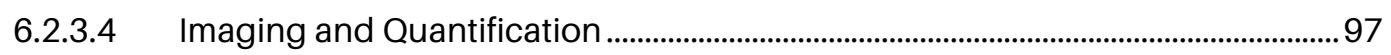

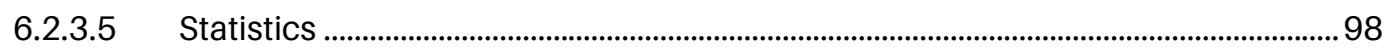

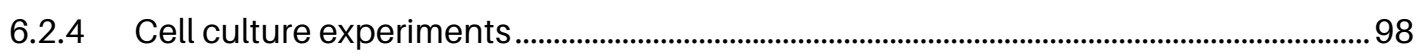

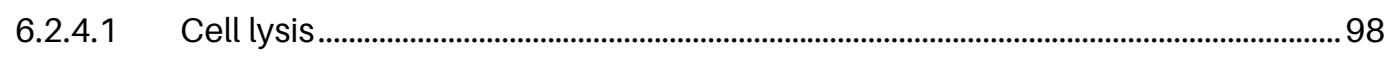

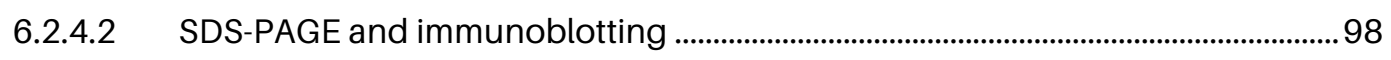

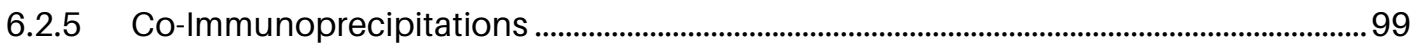

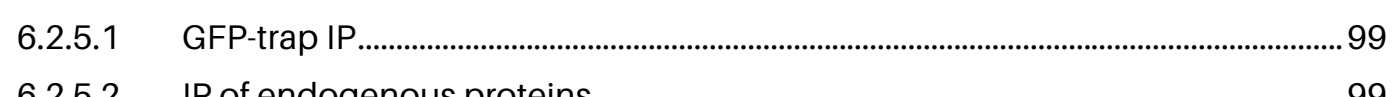

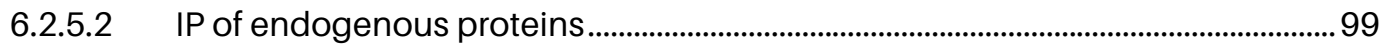

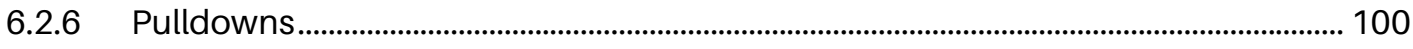

6.2.6.1 His-ubiquitin pulldown ........................................................................................... 100

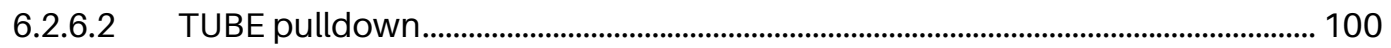




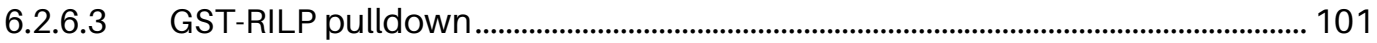

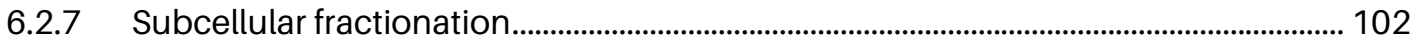

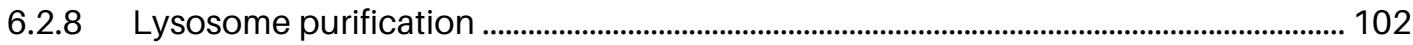

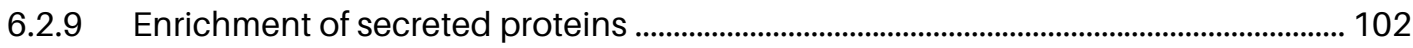

6.2.10 Mass spectrometry experiments.............................................................................................. 103

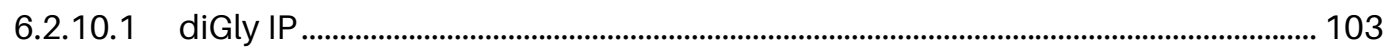

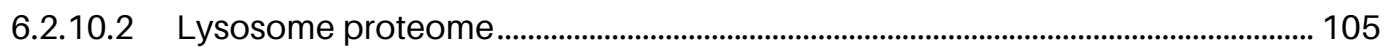

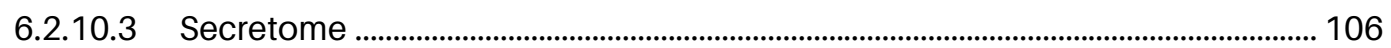

6.2.10.4 LAMTOR1 ubiquitination sites and interactome ..................................................... 106

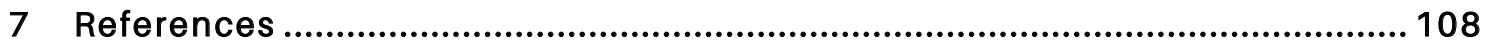

VII. Acknowledgments......................................................................... 119

VIII. Curriculum Vitae.............................................................................. 120 


\section{List of abbrevations}

\begin{tabular}{|c|c|}
\hline AA & acetic acid \\
\hline$A B C$ & ammonium bicarbonate \\
\hline ACN & acetonitrile \\
\hline AMBRA1 & activating molecule in BECN1-regulated autophagy protein 1 \\
\hline AMSH & Associated Molecule with the SH3-domain of STAM \\
\hline APS & Ammonium peroxydisulphate \\
\hline ATP & adenosine triphosphate \\
\hline BafA1 & Bafilomycin A1 \\
\hline BSA & Bovine serum albumin \\
\hline CAA & Chloroacetamide \\
\hline Cas9 & CRISPR associated protein 9 \\
\hline Cath / CTS & cathepsin \\
\hline Cl-M6PR & cation-indipendent mannose-6-phosphate receptor \\
\hline CRC & cargo recognition complex \\
\hline $\begin{array}{l}\text { CRISPR } \\
\text { ctrl }\end{array}$ & $\begin{array}{l}\text { Clustered Regularly Interspaced Short Palindromic Repeats } \\
\text { control }\end{array}$ \\
\hline DMEM & Dulbecco's Modified Eagle Medium \\
\hline DMSO & Dimethyl sulfoxide \\
\hline DNA & deoxyribonucleic acid \\
\hline DTT & Dithiothreitol \\
\hline DUB & deubiquitinase, deubiquitinating enzyme \\
\hline DUSP & domain present in ubiquitin specific proteases \\
\hline EBSS & Earle's Balanced Salt Solution \\
\hline EBSS & Earle's Balanced Salt Solution \\
\hline EDTA & Ethylenediaminetetraacetic acid \\
\hline $\mathrm{EE}$ & early endosome \\
\hline EGF & epidermal growth factor \\
\hline EGFR & epidermal growth factor receptor \\
\hline ELY & endolysosome \\
\hline ESCRT & endosomal sorting complexes required for transport \\
\hline $\mathrm{EtOH}$ & ethanol \\
\hline FBS & fetal bovien serum \\
\hline $\mathrm{fl}$ & full length \\
\hline FYCO1 & FYVE and coiled-coil domain containing protein 1 \\
\hline GAP & GTPase-activating protein \\
\hline GDP & Guanosine diphosphate \\
\hline GE & gel electrophoresis \\
\hline GEF & guanine nucleotide exchange factor \\
\hline GFP & green fluorescent protein \\
\hline gRNA & guide RNA \\
\hline GTP & Guanosine triphosphate \\
\hline $\mathrm{HCl}$ & Hydrochloric acid \\
\hline HECT & Homologous to the E6-AP Carboxyl Terminus \\
\hline HEK & human embryonic kidney \\
\hline His & histidine \\
\hline HOPS & homotypic fusion and protein sorting \\
\hline
\end{tabular}




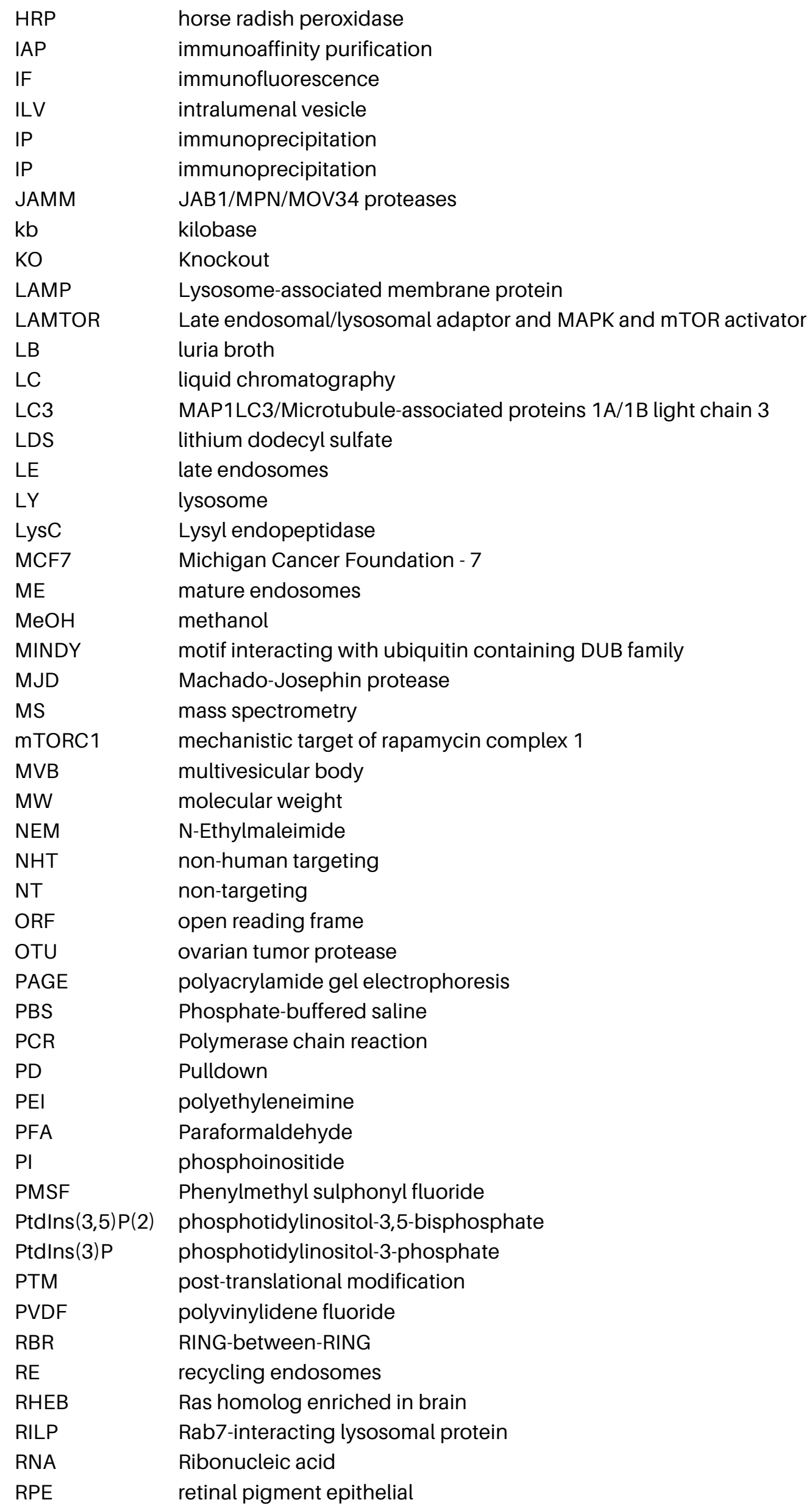




\begin{tabular}{|c|c|}
\hline RT & room temperature \\
\hline SDC & Sodium deoxycholate \\
\hline SDM & Site-directed mutagenesis \\
\hline SDS & Sodium dodecyl sulfate \\
\hline SILAC & stable isotope-labeling of amino acids in cell culture \\
\hline siRNA & small interfering RNA \\
\hline SLC38A9 & Solute Carrier Family 38 Member 9 \\
\hline SNX & sorting nexin \\
\hline TAE & tris acetate ETDA \\
\hline TBC & Tre-2/Bub2/Cdc16 \\
\hline TBS & tris-buffered saline \\
\hline TCA & trichloroacetic acid \\
\hline TE & tris-EDTA \\
\hline TFA & Trifluoroacetic acid \\
\hline TFEB & transcription factor EB \\
\hline TGN & trans-Golgi network \\
\hline TMEM192 & transmembrane protein 192 \\
\hline TRIM27 & Tripartite Motif Containing 27 \\
\hline TUBE & tandem ubiquitin binding entity \\
\hline U2OS & U2 osteosarcoma \\
\hline UBE & Ubiquitin Conjugating Enzyme \\
\hline ubi & ubiquitin \\
\hline UBL & Ubiquitin like \\
\hline $\mathrm{UCH}$ & ubiquitin C-terminal hydrolase \\
\hline ULK1 & Unc-51-like kinase \\
\hline USP & ubiqutin-specific protease \\
\hline UVRAG & UV radiation resistance-associated gene protein \\
\hline v-ATPase & Vacuolar-type ATPase \\
\hline WASH & Wiskott Aldrich Syndrome protein and scar homologue \\
\hline WB & Western blot \\
\hline WB & Western blot \\
\hline WIPI & WD repeat domain phosphoinositide-interacting protein \\
\hline wt & wildtype \\
\hline ZUFSP & Zinc finger-containing ubiquitin peptidase \\
\hline
\end{tabular}




\section{List of figures}

Figure 1-1: Overview of the endosomal-lysosomal system ……………………………………………..... 22

Figure 1-2: Endosome maturation................................................................................................................ 23

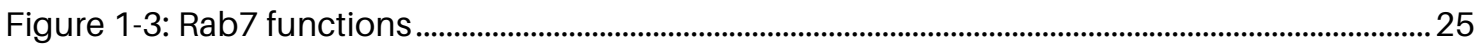

Figure 1-4: mTORC1 acitvation at the lysosomal surface ..................................................................2 27

Figure 1-5: mTORC1 regulation of transcription and autophagy. .......................................................2

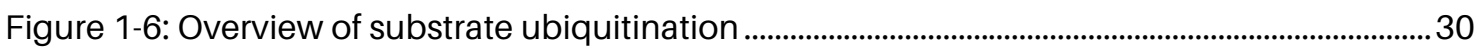

Figure 1-7: DUB families .........................................................................................................................

Figure 1-8: Overview of deubiquitination .............................................................................................

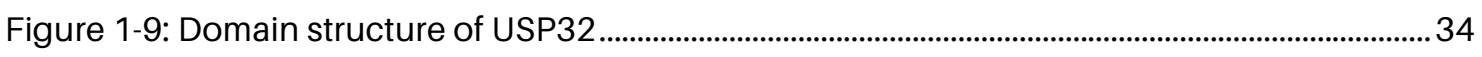

Figure 3-1: Expression levels and depletion of USP32 in different cell types....................................37

Figure 3-2: Different USP32 constructs were designed and expressed in cells.................................39

Figure 3-3: USP32 is localized at membranes in the perinuclear region of cells................................ 40

Figure 3-4: Ubiquitinome analyses determined potential USP32 substrates involved in endosomal trafficking as well as lysosomal proteins. ......................................................... 42

Figure 3-5: USP32 cleaves a non-proteolytic signal from endosomal-lysosomal proteins. ........... 43

Figure 3-6: Rab7 shows interaction and USP32-sensitive ubiquitination .............................................. 44

Figure 3-7: USP32-sensitive Rab7 ubiquitination alters Rab7 activity but not its localization. .... 45

Figure 3-8: Rab7 mediated retrograde trafficking of CI-M6PR is enhanced upon USP32

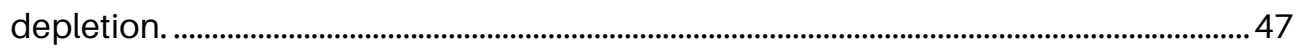

Figure 3-9: Sorting of lysosomal enzymes is impaired in USP32-deficient cells.................................50

Figure 3-10: Enhanced secretion of lysosomal hydrolases is not detected...........................................52

Figure 3-11: USP32 does not directly affect retromer cargo recognition complex...........................54

Figure 3-12: USP32 interacts with its possible substrate LAMTOR1. ..................................................... 56

Figure 3-13: LAMTOR1 ubiquitination is USP32-sensitive. ..................................................................... 58

Figure 3-14: Lysosomal LAMTOR1 localization is partially lost in USP32KO cells..............................59

Figure 3-15: USP32 does not regulate lysosome distribution in cells..................................................60

Figure 3-16: Interaction of LAMTOR1 and lysosomal v-ATPase subunits is weaker upon USP32 depletion. ...................................................................................................................................62

Figure 3-17: Lysosomal mTOR localization is partially lost in USP32KO cells.....................................63

Figure 3-18: Phosphorylation of mTORC1 substrates is decreased after reactivation in USP32KO cells.

Figure 3-19: USP32-depleted cells show more LC3 puncta upon starvation. .....................................66

Figure 3-20: USP32 depletion impairs autophagic flux. ............................................................................68

Figure 4-1: Model for the regulation of Rab7 function by USP32 ....................................................69

Figure 4-2: Model for the regulation of LAMTOR1 and Ragulator function by USP32 ................... 70

Figure 5-1: Models of v-ATPase-Ragulator-Rag GTPases-mTORC1 interactions.............................79 


\section{List of tables}

Table 3-1: Tested conditions for USP32 expression

Table 3-2: Proteins and complexes involved in the regulation of mTORC1 activity at lysosomes 60

Table 6-1: CellProfiler pipeline for dot counting.....................................................................................97

Table 6-2: CellProfiler pipeline for co-localization and dot dispersion 97 


\section{Declarations}

Except where stated otherwise by reference or acknowledgment, the work presented was generated by myself under the supervision of my advisors during my doctoral studies. All contributions from colleagues are explicitly referenced in the thesis. The material listed below was obtained in the context of collaborative research:

Figure 3-1B: Generation of U2OS and RPE1 USP32 knockout cell lines using CRISPR/Cas9, Verena Bittl (AG Bremm, Institute of Biochemistry II, Goethe University Frankfurt) generated the cell lines and performed the Western blot, I initiated the generation of the cell line

Figure 3-4 B: SILAC-based ubiquitinome analysis to identify USP32 substrates, Florian Bonn (Institute of Biochemistry II, Goethe University Frankfurt) performed the LC-MS/MS analysis and data processing, I performed the experiment (cell culture, diGly IP, sample preparation for MS)

Figure 3-7: Rab7 localization and RILP binding, Florian Steinberg (Center for Biological Systems Analysis, University of Freiburg) performed the immunofluorescence experiment and GST-RILP pulldown, I initiated the collaboration after having technical issues with the same experiment, and prepared the figure

Figure 3-8: Rab7 mediated retrograde Cl-M6PR transport, Florian Steinberg (Center for Biological Systems Analysis, University of Freiburg) performed the immunofluorescence experiment, I initiated the collaboration after having technical issues with the same experiment, and prepared the figure

Figure 3-9 B, C: SILAC-based lysosome proteome analysis, Florian Bonn (Institute of Biochemistry II, Goethe University Frankfurt) performed the LC-MS/MS analysis and data processing, I performed the experiment (cell culture, lysosome enrichment, sample preparation for MS)

Figure 3-10 B: SILAC-based secretome analysis, Florian Bonn (Institute of Biochemistry II, Goethe University Frankfurt) performed the LC-MS/MS analysis and data processing, I performed the experiment (cell culture, enrichment of secreted proteins, sample preparation for MS)

Figure 3-13 A: Label-free approach to analyze LAMTOR1 ubiquitination, Georg Tascher (Institute of Biochemistry II, Goethe University Frankfurt) performed the LC-MS/MS analysis and data processing, I performed the experiment (cell culture, GFP-LAMTOR1 IP, sample preparation for MS)

Figure 3-16: Label-free interactome studies, Georg Tascher (Institute of Biochemistry II, Goethe University Frankfurt) performed the LC-MS/MS analysis and data processing, I performed the experiment (cell culture, GFP-LAMTOR1 IPs, sample preparation for MS) 


\section{Zusammenfassung}

Die Aufrechterhaltung zellulärer Prozesse erfordert einen regulierten gerichteten Transport von Proteinen und Membranen. Eine zentrale Rolle in diesem Vorgang hat das endosomale und lysosomale System mit seinen membrangebundenen Organellen und Vesikeln. Hier findet der größte Teil der Sortierung und des Transports von Proteinen zu ihrem Ziel in der Zelle statt. Damit ist dieses dynamische Netzwerk essentiell für die Regulation von verschiedensten Signalen und metabolischen Prozessen. Das System besteht aus mehreren Kompartimenten mit verschiedenen Charakteristiken und Funktionen: frühe Endosomen, Recycling-Endosomen, späte Endosomen, Lysosomen und im erweiterten Sinne auch Autophagosomen.

Endosomale Kompartimente haben zwar unterschiedliche Eigenschaften, sind aber durch einen Reifeprozess in einem fließenden Übergang mit morphologischen wie auch molekularbiologischen Veränderungen miteinander verbunden.

Eine essentielle Schlüsselrolle für späte Endosomen hat Rab7. Die GTPase bindet in ihrem aktiven GTP-gebundenen Zustand Effektor-Proteine und ist damit in die Reifung von frühen zu späten Endosomen, die koordinierte Verschmelzung von frühen mit späten endosomalen Vesikeln als auch von späten Vesikeln mit Lysosomen oder Autophagosomen, sowie den anterograden und retrograden Transport im endosomalen System involviert. Des Weiteren hilft Rab7 dem Recycling von Sortierungs-Rezeptoren vom endosomalen Kompartiment zurück zum trans-Golgi Netzwerk (TGN). Solche Rezeptoren, wie CI-M6PR, verhelfen der richtigen Sortierung von lysosomalen Proteinen und müssen nach Entlassen ihrer „Fracht" durch retrograden Transport mittels des Retromer-Komplexes wieder verfügbar gemacht werden.

Alle beschrieben Prozessen, in die Rab7 involviert ist, spielen auch eine Rolle für die Bildung und Funktion von Lysosomen. Lysosomen sind oft der Endpunkt vieler Zellprozesse, da sie mit ihrem sauren $\mathrm{pH}$-Wert und enthaltenen Hydrolasen (die Fracht der Rezeptoren) den Abbau von Proteinen übernehmen. Die abzubauenden Proteine werden auf verschiedenen Wegen über z.B. Endozytose oder Autophagie zu den Lysosomen transportiert. Allerdings sind Lysosomen nicht nur die "Müllverwerter" der Zelle, sondern fungieren auch als dynamisches Zentrum für verschiedenste metabolische Signale. Wie ein Messfühler erkennen Lysosomen den Nährstoffe-Status in der Zelle, passen sich diesem an und geben das ermittelte Signal an nachfolgende Komplexe weiter. Einer dieser Komplexe ist mTORC1, einer der wichtigsten Regler biosynthetischer Wege. mTORC1 bindet dynamisch als Reaktion auf verfügbaren Aminosäuren an die lysosomale Oberfläche, wo er als Kinase aktiviert wird. Dieser Prozess besteht aus mehreren komplexen und einhergehenden Schritten in Abhängigkeit von Aminosäure-Verfügbarkeit im und um das Lysosom. Die lysosomalen Aminosäure-Sensoren v-ATPase und SLC38A9 erkennen die Nährstoff-Konzentration und vermitteln dies an den Ragulator-Komplex (bestehend aus LAMTOR1-5). Ragulator aktiviert die nachfolgenden Rag GTPasen. Daraufhin wird mTORC1 aus dem Zytoplasma zur Lysosomen-Membran rekrutiert, wo mTORC1 aktiviert wird. Bei sinkenden Aminosäure-Level wechseln die Komplexe wieder in ihren inaktiven Zustand, sodass inaktiver mTORC1 von der Membran ins Zytoplasma entlassen 
wird. Durch seine Kinaseaktivität inhibiert mTORC1 unter anderem den Prozess der Autophagie.

So wie Ubiquitin-Signale an nahezu allen zellulären Prozessen beteiligt sind, wurde ihre regulatorische Rolle auch für das endosomale-lysosomale System und die mTORC1 Signalübertragung gezeigt. Deubiquitinasen (DUBs) kotrollieren dabei den genauen Ausgang der Ubiquitin-Modifikation, indem sie das Signal anpassen oder gänzlich beenden. Beispielhaft kann hier die Ubiquitinierung des EGF Rezeptors genannt werden, die zur Internalisierung und dem Abbau des Rezeptors im Lysosom führt. Deubiquitinierung des EGF Rezeptors ermöglicht dessen Recycling und Rücktransport zur Zellmembran. Darüberhinaus wird durch Ubiquitin und DUBs auch der Retromer-vermittelte Transport oder die zelluläre Reaktion auf eine Beschädigung der Lysosomen reguliert.

Die Ubiquitin-spezifische Protease 32 (USP32) ist eine Deubiquitinase mit bisher nicht charakterisierter zellulärer Funktion. Die Domänenstruktur von USP32 umfasst neben bekannten USP Domänen auch zwei gänzlich einzigartige Domänen unter allen DUBs, nämlich $\mathrm{N}$-terminale EF Hände (Calcium-Binde-Motiv) und eine C-terminale Prenylierungsstelle für eine mögliche Lipidierung. Zusammen mit wenigen bereits bekannten Daten, die USP32 als mögliches Onkogen in verschiedenen Krebstypen beschreiben, sowie seine Lokalisation am TGN und Interaktion mit einer Komponente des Retromer-Komplexes zeigen, ist USP32 ein interessanter Kandidat für detaillierte Studien.

Im Zuge dieser Arbeit sollte eine erste einfache Betrachtung die molekulare Häufigkeit sowie Lokalisation von endogenem USP32 aufzeigen. Weiterhin war es Aufgabe, geeignete Werkzeuge zu etablieren um die zelluläre Rolle von USP32 ohne krankheitsbezogenen Kontext zu erforschen. In einem unbefangen Massenspektrometrie-Ansatz sollten Substrate von USP32 identifiziert werden und diese in nachfolgenden Experimenten validiert und charakterisiert werden.

Die ersten Experimente dieser Thesis dienten zur Etablierung von geeigneten Werkzeugen für die definierten Ziele der Arbeit. Um negative Effekte und Schwierigkeiten einer kurzweiligen siRNA basierten Depletion von USP32 in verschiedenen Zelltypen zu umgehen, konnte mit der CRISPR/Cas9 Technologie eine stabile polyklonale USP32 Knockout (USP32KO) sowie KontrollZellinie (non-human targeting, NHT) in RPE1 und U2OS Zellen etabliert werden. Trotz vieler Bemühungen war es leider nicht möglich eine robuste Expression von exogenem getaggtem USP32 als weiteres Werkzeug zu erzielen. Daher wurde beschlossen, eine Überexpression nur für wenige ausgewählte Experimente vorzunehmen. Weitere Experimente zeigten, dass USP32 unterschiedliche Expressionslevel in verschiedenen Zelllinien aufweist, wobei die erhöhten Proteinlevel in MCF7 Brustkrebszellen herauszustellen ist. Darüberhinaus konnte die subzelluläre Lokalisation von endogenem USP32 im Zytoplasma als auch in geringerem Maße an Membranen des trans-Golgi Netzwerks und Lysosomen detektiert werden.

Für nachfolgende Experimente zur zellulären Charakterisierung von USP32 ohne Krankheitshintergrund wurden bevorzugt RPE1 Zellen (aufgrund ihrer Eigenschaften ähnlich von Primärzellen) mit USP32KO verwendet. Die Ergebnisse in RPE1 Zellen wurden ggf. in U2OS Zellen reproduziert. Für die Identifizierung von USP32 Substraten wurde ein unvoreingenommener Massenspektrometrie-Ansatz gewählt. Hierbei wurden zur späteren 
semi-quantitativen Analyse Proteine in RPE1 NHT und USP32KO Zellen durch SILAC (stable isotope labeling with amino acids in cell culture) markiert. Nach tryptischem Verdau wurden solche Proteine identifiziert, die in USP32KO Zellen erhöhte Ubiquitinierung aufwiesen. Interessanterweise befanden sich unter diesen Treffern Proteine aus dem endosomalen und lysosomalen System mit folgenden modifizierten Lysin-Resten: Rab7 K191/194 (C-Terminus), LAMTOR1 K20 (N-Terminus), Rab11 K179 (C-Terminus), TMEM192 K249 (C-Terminus). Die Proteinlevel der vier Kandidaten waren sowohl in der Proteom-Analyse der diGly IP als auch in der folgenden Immunoblot-Analyse bei USP32 Depletion unverändert. Dies deutete daraufhin, dass USP32 kein proteolytisches Signal an diesen vier Proteinen schneidet und somit nicht direkt deren Stabilität bzw. proteasomalen Abbau kontrolliert.

Wie vorher bereits beschrieben, hat Rab7 eine essentielle Rolle für Endosomen und Lysosomen. Daher wurde die Modifikation an Rab7 als erstes Substrat in nachfolgenden Studien betrachtet. Rab7 und USP32 co-immunpräzipitierten, was die Interaktion von Substrat und Enzym zeigte. Nach Überexpression von His-getaggtem Ubiquitin und Rab7 mit oder ohne USP32 in HEK293 Zellen wurde eine vermehrte Modifikation von Rab7 ohne gleichzeitiges Vorhandensein von exogenem USP32 festgestellt, was die USP32-sensitive Ubiquitinierung bestätigte. Im weiteren Verlauf der Arbeit konnte keine Veränderung der subzellulären Lokalisation von Rab7 an lysosomalen Strukturen erkannt werden. Allerdings resultierte die vermehrte Ubiquitinierung von Rab7 in RPE1 USP32KO Zellen zu einer stärkeren Bindung von seinem Effektor-Protein. Da RILP an aktives GTP-gebundenes Rab7 bindet, wurde geschlussfolgert, dass die Aktivität von ubiquitiniertem Rab7 oder die Affinität von RILP zu ubiquitiniertem Rab7 erhöht war. Die Konsequenzen dieser erhöhten Aktivität wurden anhand des bekannten, Rab7 abhängigen Modell-Transports von CI-M6PR genauer untersucht. Dazu wurden RPE1 NHT und USP32KO Zellen mit einem Antikörper gegen CI-M6PR inkubiert und die Internalisierung des Antikörpers nach verschiedenen Zeitpunkten mittels Immunfluoreszenz-Mikroskopie beobachtet. Die Co-Lokalisation des Rezeptors mit dem transGolgi Marker TGN46 war nach 15 min in USP32-depletierten Zellen erhöht. Ein ähnliches Bild wurde unter stationären Bedingungen beobachtet, wobei die zelluläre Gesamt-Lokalisation von CI-M6PR an Giantin, einem weiteren TGN Marker, in RPE1 USP32KO Zellen ebenfalls gesteigert war. Daher schien der Rücktransport von Cl-M6PR zum trans-Golgi Netzwerk schneller bzw. effizienter in Abwesenheit von USP32. Als weiteres Indiz für einen begünstigten Rezeptor-Transport wurden angereicherte Mengen an Hydrolasen in isolierten Lysosomen aus USP32-depletierten U2OS Zellen mittels SILAC Massenspektrometrie detektiert. Zur Verifikation wurden ein komplementärer Western Blot durchgeführt und das MassenspektrometrieErgebnis konnte bestätigt werden.

Zusätzlich zu der Untersuchung von Rab7 als USP32 Substrat wurde auch der Einfluss von USP32 auf den Retromer-Komplex, genauer auf dessen trimeren Cargo Recognition Complex (CRC), betrachtet. USP32 co-immunpräzipitierte mit allen drei Komponenten. Der Knockout von USP32 in RPE1 und U2OS Zellen zeigte jedoch keinen Einfluss auf die Proteinlevel oder auf die Komplex-Zusammensetzung.

Zusammenfassend lässt sich schlussfolgern, dass USP32 Rab7 an K191/194 deubiquitiniert und somit dessen Aktivität und Bindung zu Effektoren moduliert. Dies resultiert in angepasstem 
Transport von lysosomalen Hydrolasen mit dem Cl-M6PR ohne direkten Einfluss auf den beteiligten Retromer-Komplex.

Mit den bisher beschriebenen Ergebnissen wurde angenommen, dass USP32 nicht nur eine Rolle im endosomalen System spielt sondern auch auf Lysosomen einwirkt. Damit einhergehend wurde als zweites Substrat LAMTOR1 weiter charakterisiert. LAMTOR1 ist Teil des pentameren Ragulator-Komplexes. Der innere „Kern” aus LAMTOR2-5 wird von LAMTOR1 umwickelt und durch dessen $\mathrm{N}$-terminale Lipidierung an die lysosomale Membran verankert. Wie bereits vorher erklärt, ist der Ragulator-Komplex ein wichtiges Bindeglied zwischen dem initialen Aminosäure-Signal und mTORC1 Rekrutierung und verhilft zur Aktivierung des KinaseKomplexes. Also ist die Steuerung von LAMTOR1 und Ragulator Funktion über posttranslationale Modifikationen wie Ubiquitin von Bedeutung. Ähnlich wie für Rab7 wurde in ersten Experimenten über Co-Immunpräzipitation gezeigt, dass LAMTOR1 und USP32 interagierten. Die diGly IP zeigte eine USP32-abhängige Modifikation von LAMTOR1 am Lysin 20. Dies konnte mittels Massenspektrometrie durch eine GFP-LAMTOR1 Anreicherung aus RPE1 NHT und USP32KO Zellen bestätigt werden. Darüberhinaus waren K31 und K60 in USP32KO Situation auch vermehrt ubiquitiniert, allerdings nicht signifikant. In einem weiteren His-Ubiquitin Pulldown Experiment zeigte sich, dass LAMTOR1 USP32-sensitiv ubiquitiniert ist (mehr modifiziertes LAMTOR1 Wildtyp bei Co-Expression mit katalytisch inaktivem USP32 in HEK Zellen), aber K20 keinen größeren Einfluss auf die gesamte Modifikation von LAMTOR1 hatte bzw. auch andere Lysinreste von USP32 deubiquitiniert werden könnten (gleiches Modifikationsmuster von LAMTOR1 K20 wie Wildtyp mit aktivem oder inaktivem USP32). Des Weiteren wurden ubiquitinierte Proteine aus RPE1 NHT und USP32KO Zellen mit TUBES (tandem ubiquitin binding entities) angereichert. Die Modifikation von LAMTOR1 war erhöht und die Level von unmodifiziertem LAMTOR1 niedriger in USP32KO Situation, was sich nach Aminosäure-Depletion mit EBSS Medium nicht änderte. Natürlich stellte sich damit die Frage, welche Funktion die Ubiquitinierung von K20 hatte. Dieser Frage wurde als Erstes mit Hilfe von Immunfluoreszenz in RPE1 und U2OS Zellen nachgegangen. Die LAMTOR1 Lokalisation an LAMP2-positiven Lysosomen war unabhängig von zellulären Aminosäure-Level erniedrigt in Abwesenheit von USP32. Eine Beeinflussung der LAMTOR1-vermittelten subzellulären Positionierung von Lysosomen konnte aber nicht erkannt werden.

LAMTOR1 und Ragulator interagieren zur Signalübertragung auf mTORC1 auf dynamische Weise mit verschiedenen Proteinen und Komplexen. Um einen möglichen Effekt auf diese Interaktionen zu untersuchen, wurde eine Co-Immunpräzipitation von GFP-LAMTOR1 aus unmarkierten RPE1 NHT und USP32KO Zellen durchgeführt und mittels Massenspektrometrie ein Interaktom erstellt. Die Zusammensetzung des Ragulator Komplexes war unverändert. Aber die reduzierte Interaktion mit Untereinheiten der lysosomal v-ATPase nach USP32 Depletion fiel auf. Diese Interaktion ist entscheidend für die nachfolgende mTORC1 Rekrutierung und Aktivierung. Folgerichtig wurde daher in weiteren Experimenten die Lokalisation und Aktivität von mTORC1 untersucht. Mittels Immunfluoreszenz wurde herausgefunden, dass mTOR (mTORC1 Untereinheit) in RPE1 USP32KO Zellen unabhängig von verfügbaren Nährstoffen weniger an Lysosomen lokalisiert war. Die Aktivität von mTORC1 wurde per Western Blot betrachtet, wobei Antikörper spezifisch gegen die phosphorylierte Form von direkten mTORC1 
Substraten verwendet wurden. RPE1 NHT und USP32KO Zellen wurden nach langer Aminosäure-Depletion mit EBSS Medium wieder in volles Zellmedium gebracht und danach die Re-Phosphorylierung über Zeit ausgewertet. Ohne USP32 war die Phosphorylierung von ULK1, WIPI2 und AMBRA1 nach Reaktivierung niedriger, was darauf schließen lies, dass mTORC1 eine geringere Aktivität in Abwesenheit von USP32 hatte.

Die Ergebnisse für die USP32-sensitive LAMTOR1 Ubiquitinierung wiesen darauf hin, dass USP32 LAMTOR1 an K20 deubiquitiniert. Dadurch wird die verstärkte Interaktion mit der v-ATPase ermöglicht und die mTORC1 Aktivierung moduliert.

Sowohl Rab7 als auch mTORC1 haben eine wichtige Rolle im Prozess der Autophagie, indem sie den Vesikeltransport vermitteln, und die Induktion sowie Transkription von Autophagieassoziierten Proteinen regulieren. Da USP32 in beide Zellwege involviert zu sein schien, wurde in einer abschließenden Untersuchung der Einfluss von USP32 Depletion auf Autophagie betrachtet. Mit Immunfluoreszenz-Mikroskopie und Western Blot wurde die Menge des Autophagie-Markers LC3 detektiert. Ein Anstieg in LC3 Punkten sowie lipidiertem LC3 nach Autophagie Induktion mit EBSS Medium in RPE1 und U2OS Zellen ohne USP32 zeigten an, dass USP32 tatsächlich einen Sekundäreffekt auf den Autophagieprozess hat.

Mit der vorliegenden Arbeit konnten Teile der zellulären Funktion der Deubiquitinase USP32 charakterisiert werden. Zusammen mit einer konkurrierenden Publikation wurde die Regulation von Rab7 durch USP32-sensitive Ubiquitinierung beschrieben. Als erste Studie zeigte die vorliegende Arbeit jedoch die Regulation vom LAMTOR1 und Ragulator durch eine Deubiquitinase. Die erweiterte Rolle von USP32 im endosomalen und lysosomalen System trägt zum Verständnis des Ubiquitin Signals in diesem Zusammenhang bei und kann Ausgangspunkt für nachfolgende Projekte in verschiedenen Richtungen der molekularbiologischen Grundlagenforschung als auch der Erforschung von assoziierten Krankheiten, wie neurodegenerative Krankheiten, sein. 


\section{Summary}

The regulation of essential cellular processes requires tightly controlled and directed transport of proteins and membranes. The highly dynamic endosomal and lysosomal system forms the key network for exchange and trafficking of molecules with its early endosomes, recycling endosomes, late endosomes, lysosomes, and additionally autophagosomes.

In this system, the small GTPase Rab7 has an essential role at the late endosomal stage regulating vesicle transport, tethering, and fusion, and retromer mediated receptor recycling back to the trans-Golgi network (TGN). Thus, Rab7 is also important for autophagosomes and lysosomes.

Lysosomes do not only represent the end point of the degradation pathway with several feeder pathways. But these organelles are also a dynamic signaling hub for a variety of metabolic processes. The ever-important regulator of cellular biosynthetic pathways mTORC1 dynamically associates with lysosomes where it is activated. mTORC1 activation is a complex multi-step process where a series of signaling events converge in dependence of amino acid levels thereby enabling interactions between the lysosomal v-ATPase, Ragulator complex (consisting of LAMTOR1-5), and Rag GTPases.

Ubiquitin signals are involved in almost all cellular processes. With this, their regulatory mechanism is also described for the endosomal-lysosomal system as well as mTORC1 signaling. Deubiquitinases (DUBs) release conjugated ubiquitin from proteins and thereby maintain the dynamic state of the cellular ubiquitinome.

The ubiquitin-specific protease 32 (USP32) is a poorly characterized DUB with only emerging cellular function. However, its predicted domain structure includes two unique domains within the entire DUB family. It has been linked to the development of breast cancer and small cell lung cancer. Furthermore, overexpressed GFP-USP32 was localized the TGN, and a global mass spectrometry-based DUB interactome study suggested an interaction with the retromer complex. Based on these data, USP32 was a very interesting candidate to study its cellular function in this PhD project.

To investigate the function without disease background, a polyclonal USP32 knockout (USP32KO) RPE1 cell line was generated using the CRISPR/Cas9 technology. First experiments revealed different protein expression levels in various cell lines, and a subcellular localization of USP32 at membranes of the Golgi and lysosomal compartments. In a subsequent SILAC-based ubiquitinome analysis potential substrates of USP32 were identified. Interestingly, various proteins of the endosomal-lysosomal system were detected with enriched non-proteolytic ubiquitination upon USP32 depletion.

The further characterization of Rab7 as USP32 substrate confirmed the USP32-sensitive ubiquitination of Rab7 at lysine (K) residues 191 and 194. The ubiquitination in USP32KO cells did not change the subcellular localization of Rab7, but enhanced the interaction with the effector protein RILP. This implied that Rab7 was either more active or RILP had higher affinity to ubiquitinated Rab7. The subsequent results verified this theory. The retromer mediated recycling of CI-M6PR back to the TGN was faster or more efficient in USP32-depleted cells. 
Accompanying this, levels of hydrolases were enriched in lysosomes isolated from USP32KO cells. Notably, USP32 had no direct effect on expression level or assembly of the retromer complex itself.

The observed lysosomal phenotypes connected another identified substrate to the function of USP32 in the endosomal-lysosomal system: LAMTOR1. LAMTOR1 is a component of the Ragulator complex and thus involved in the activation of mTORC1 at the lysosomal surface. Similar as for Rab7, the first experiments to characterize LAMTOR1 as USP32 substrate confirmed the USP32-sensitive ubiquitination at K20 independent of amino acid availability. However, ubiquitination of LAMTOR1 decreased its lysosomal localization in untreated and amino acid starved USP32KO cells. The following label-free interactome study detected a reduced interaction of LAMTOR1 and subunits of the lysosomal V-ATPase upon loss of USP32. This resulted in a shifted subcellular localization of mTOR (subunit of mTORC1) away from lysosomes. Furthermore, direct substrates of mTORC1 were less or slower re-phosphorylated after long amino acid starvation and re-activation of mTORC1 in USP32KO cells indicating a reduced mTORC1 activity.

Both USP32-dependent regulations of Rab7 and LAMTOR1/Ragulator converged in enhanced autophagic processes analyzed by increased LC3 levels upon amino acid starvation and USP32 depletion.

In summary, the presented thesis described the diverse role of USP32 in the endosomal and lysosomal system, and contributes to the understanding of novel ubiquitin signals in this context. 


\section{Introduction}

\subsection{The endosomal-lysosomal system}

The endosomal-lysosomal system is linked to most aspects of cell life by regulating essential cellular processes in signaling, dynamics and metabolic actions like protein degradation and recycling. A series of highly dynamic membrane-enclosed organelles and vesicles forms the network for exchange and trafficking of cargo molecules. Various compartments with different functions and roles have been identified: early endosomes, recycling endosomes, late endosomes, lysosomes, and additionally autophagosomes (Hu et al., 2015).

Endosomal compartments undergo maturation with morphological and biological changes. Transition from early to late endosomes and lysosomes includes decreasing of the luminal $\mathrm{pH}$, altering the phosphatidylinositol lipid composition, and differential recruitment and activation of Rab-family GTPases. The Rab GTPases specify the structural and functional identity of the organelles, and organize membrane subdomains (Elkin et al., 2016; Huotari and Helenius, 2011).

\subsubsection{Early endosomes}

The early endosomes (EEs) serve as a crucial sorting platform for lipid and protein cargo through the endocytic pathway. In the endocytic pathway, material is internalized by several routes (e.g. clathrin-coated vesicles) and delivered to early endosomes, where its fate is decided: endocytosed cargo can be efficiently sorted to late endosomes (LEs) and lysosomes for degradation, sent to the trans-Golgi network (TGN) via retrograde trafficking, or returned to the plasma membrane by direct rapid recycling or slower recycling with recycling endosomes (REs). Coordinate recruitment and assembly of sorting machineries mediates the formation of distinct microdomains within EEs for subsequent segregation. Formation of thin membrane tubules concentrates recycling cargo for the transport to the plasma membrane or TGN and transport carriers bud off the endosomal membrane. Molecules destined for lysosomal degradation cluster in flat luminal invaginations, which are pinched off as free cargo-containing intraluminal vesicles (ILVs). These multivesicular regions detach from the endosomal membrane as multivesicular bodies (MVBs) with a limiting membrane. Afterwards, MVBs mature to late endosomes. The morphological changes of the membrane architecture, the overall membrane organization and protein transport at EEs are mediated by key components such as Rab GTPases and described in the following section (Gruenberg, 2001; Huotari and Helenius, 2011).

\subsubsection{Key regulators at the early endosome}

Rab5 is the predominant small Rab GTPase at early endosomes. In its active GTP-bound form Rab5 regulates the fusion of endocytic vesicles with the EEs, the generation of phosphotidylinositol-3-phosphate (PtdIns(3)P) lipids, the phospholipids enriched at EEs and 
MVBs, and their movement along actin and microtubules tracks (Bucci et al., 1992; Murray et al., 2002; Nielsen et al., 1999).

Rab5 domains are the gateway into the early endosome where proteins destined for degradation are separated from proteins for recycling. Rab4 is the regulator of fast recycling back to the plasma membrane, and present on peripheral early sorting endosomes (van der Sluijs et al., 1992). In contrast, recycling cargo accumulates in more pericentriolar, tubular structures enriched in Rab11: the recycling endosomes. Rab11 acts distal of Rab5, but Rab5 dysfunction impairs REs (Ullrich et al., 1996).

Sorting of cargo, like receptors, for degradation is mediated by sorting signals at their cytosolic domain necessary for recruitment of the sorting machinery. Monoubiquitinated receptors are recognized by endosomal sorting complexes required for transport (ESCRT). ESCRT are the key regulators of $M V B$ formation and have a diverse roles ranging from cargo engaging to lipid bilayer-bending. A well-known example of endosomal sorting in MVBs is the downregulation of activated epidermal growth factor receptor (EGFR) (Henne et al., 2011).

Additionally to their recycling and degradation pathways, early endosomes serve as a connection between endocytic and biosynthetic routes. As described earlier, distinct subdomains and respective proteins regulate sorting into different pathways. The retrograde transport of cargo from endosomes back to the trans-Golgi network is mediated by the retromer complex and membrane tubulation (Bonifacino and Hurley, 2008). Notably, the two endosomal tubulation events for recycling or retrograde transport are defined by the maturation status of endosomes. Sorting into recycling tubular structures precedes the retromer-mediated formation of tubules (Mari et al., 2008).

The retromer complex is a highly conserved multi-subunit complex which associates with the endosomes on their cytosolic face. In mammalian cells, the complex is assembled of a stable trimeric cargo recognition complex (CRC) (consisting of VPS35, VPS26, and VPS29) and the membrane-bending sorting nexin (SNX) components (Seaman, 2012). In a first step, the CRC is recruited to endosomal membranes thereby recognizing and selecting its cargo. Simultaneously, SNX proteins promote the membrane tubulation. Subsequently, the CRC associates with defined SNX proteins and vesicles bud from the endosome. Eventually, the retromer proteins dissociate from the membrane to enable new rounds of cargo sorting. One of the most prominent retromer cargo is the cation-independent mannose-6-phosphate receptor (Cl-M6PR). Cl-M6PR cycles between trans-Golgi network, endocytic compartment and plasma membrane. The retromer complex mediates the return of unoccupied CI-M6PR to the TGN and allows sorting of newly synthesized hydrolases to acidic late endosomes where the cargo, lysosomal hydrolases, dissociates (Seaman, 2004, 2012; Wang and Bellen, 2015). 


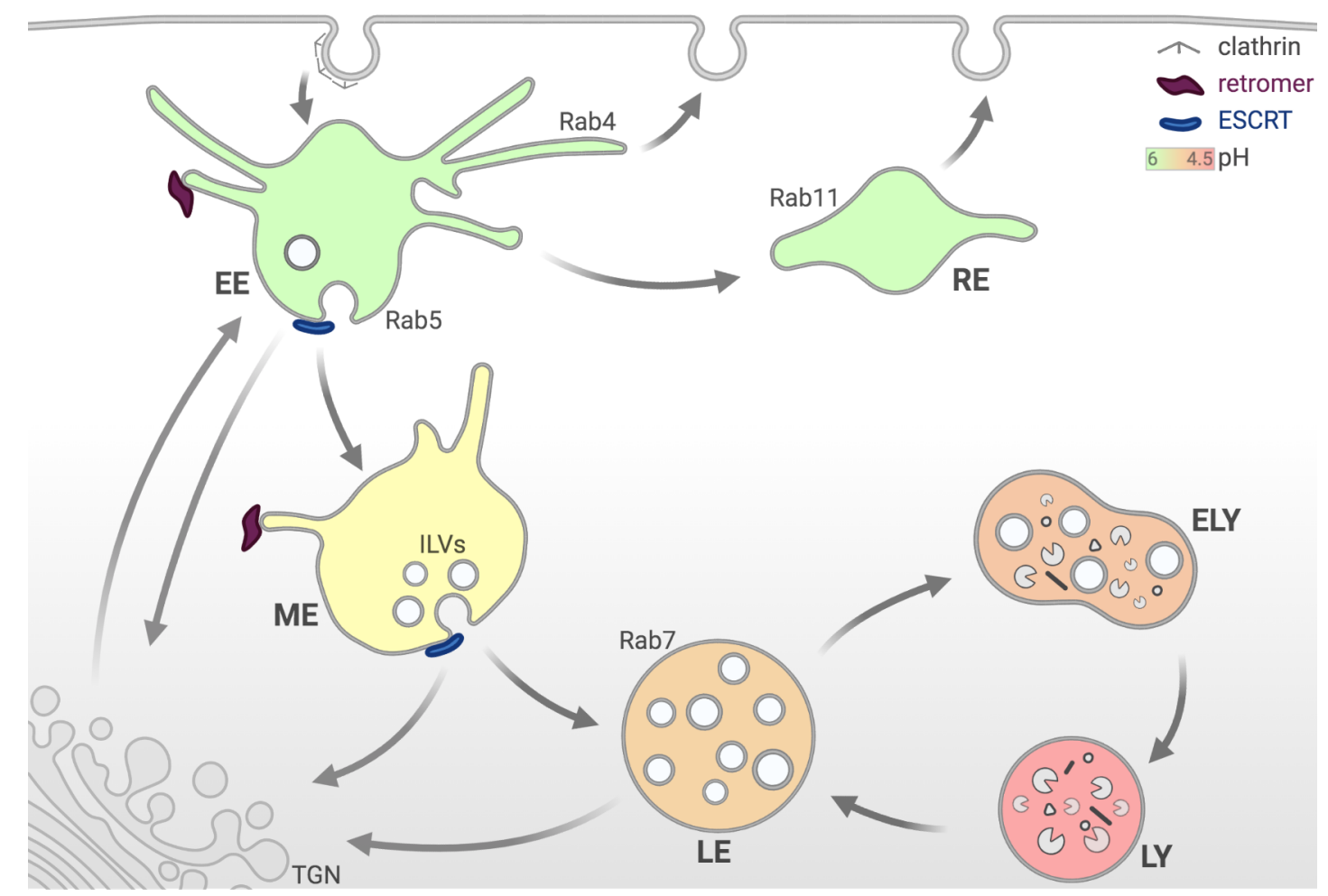

Figure 1-1: Overview of the endosomal-lysosomal system After clathrin-dependent or -independent endocytosis from the plasma membrane, vesicles fuse with early endosomes (EE) decorated with Rab5. Subsequently, cargo is sorted for recycling to the plasma membrane (directly Rab4-dependent, or indirectly in Rab11 positive recycling endosomes (RE)), for retromer-mediated transport back to the trans-Golgi network (TGN), or for degradation in intraluminal vesicles (ILV) via ESCRT. During further maturation to mature endosomes (ME) and late endosomes (LE), more ILVs form and Rab5 is exchanged with Rab7. Eventually, late endosomes fuse with lysosomes (LY) to endolysosomes (ELY) where the cargo is degraded.

\subsubsection{Maturation of endosomes}

The maturation of early to late endosomes is a tightly coordinated and regulated process and involves a multitude of changes including the following:

(I) The phosphoinositide (PI) conversion in endosome maturation plays a crucial role. Early endosomal membranes are enriched in Ptdlns(3)P lipids, whereas late endosomes contain mostly phosphotidylinositol-3,5-bisphosphate (Ptdlns $(3,5) \mathrm{P}(2))$. Since a variety of endosomal complexes and regulators have PI binding domains, Pls control membrane domains, sorting, reformation, and trafficking mediators (Poteryaev et al., 2010; Vicinanza et al., 2008).

(II) Formation of new intraluminal vesicles (ILVs) is not only critical for sorting of cargo for degradation. The process inactivates receptors by disconnecting them from the cytosol. Furthermore, ILV membranes lack protective glycosylated proteins, such as lysosomalassociated membrane proteins (LAMPS). Thus, proteins in ILVs are easily accessible for lysosomal proteases (Huotari and Helenius, 2011; Kobayashi et al., 2002).

(III) The luminal $\mathrm{pH}$ of early and late endosomes is acidic and drops from values above $\mathrm{pH} 6$ to pH 6.0-4.9 in LEs and around pH 4.5 in lysosomes. The membrane-associated v-ATPase pumps cytosolic protons into their lumen and is primarily responsible for the establishment and maintenance of the acidic $\mathrm{pH}$ of endosomal vesicles. The acidification provides an optimal environment for hydrolases, supports membrane trafficking, sorting of cargo and the release of receptor ligands (Huynh and Grinstein, 2007; Maxfield and Yamashiro, 1987; Mellman, 1996). 
(IV) A switch of motility during maturation is induced by the association of endosomes with different motor proteins on microtubules. Early endosomes undergo slow, short-ranged movements in the periphery. Those numerous small EEs are later during maturation subject to fast, long-ranged movements toward the perinuclear region and are eventually replaced by fewer, large, and roundish LEs (Aniento et al., 1993; Ballabio and Bonifacino, 2020; Collinet et al., 2010).

(V) The small GTPases Rab5 and Rab7 and their effectors determine the proper function of late and early endosomes, respectively. The maturation program involves a conversion from Rab5 to Rab7 (Jovic et al., 2010; Méresse et al., 1995; Poteryaev et al., 2010; Ullrich et al., 1994). A model for the essential Rab5-to-Rab7 switch describes Rab5 as Rab7 activator. Subsequently, Rab7 autoactivates itself and suppresses Rab5. Rab5 is removed and Rab7 take-over is initiated (Del Conte-Zerial et al., 2008; Rink et al., 2005).

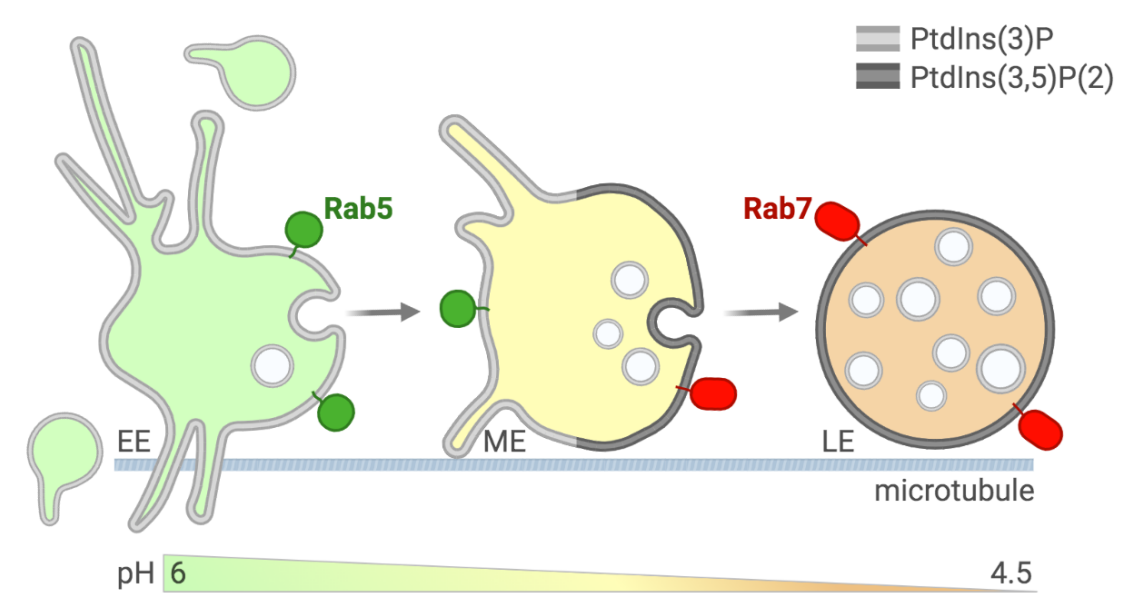

Figure 1-2: Endosome maturation The maturation of early to late endosomes involves a Rab5-to-Rab7 switch, the formation of more ILVs, a PI conversion of PtdIns(3)P to PtdIns $(3,5) \mathrm{P}(2)$, the drop of the intraluminal $\mathrm{pH}$, and transport on microtubules from the peripheral to the perinuclear region.

\subsubsection{Late endosomes and endolysosomes}

The maturing endosomes are formed in the cell periphery as roundish structures with a limiting membrane and low electron density. Another hallmark of maturing and late endosomes is the presence of numerous ILVs. The number of ILVs in those multivesicular bodies increases during maturation (Piper and Luzio, 2001). LEs are decorated with Rab7, which is a regulator of membrane trafficking and interacts with a variety of downstream effectors such as the retromer complex (Hutagalung and Novick, 2011; Rojas et al., 2008).

Along their journey to the perinuclear region, LEs fuse with each other to eventually undergo transient fusion and fission events with lysosomes to form a hybrid endolysosome. This "kissand-run" model explains the blurred definition borders between endosomes and lysosomes given that all lysosomal proteins are also found in LEs (Luzio et al., 2007; Mullock et al., 1998). The limiting membrane contains highly sialylated LAMPs giving rise to protection against the intraluminal hydrolases (Bissig and Gruenberg, 2014). They share an acidic pH between 6.0-4.9 (Maxfield and Yamashiro, 1987). But in contrast, classical lysosomes have a spherical electrondense structure, high hydrolase levels, and lack mannose-6-phosphate receptors (de Duve, 2005; Luzio et al., 2007). 


\subsubsection{Rab7 as key regulator of late endosomes and autophagy}

Rab7 coordinates directly or indirectly almost every event within the course of maturation from early to late endosomes and lysosomes. Belonging to the family of small GTPases and enriched in late endosomes, it is a key regulator of formation, trafficking, and fusion of transport vesicles (BasuRay et al., 2012). Mediated by its C-terminal prenylation Rab7 cycles between a cytosolic and a membrane-attached state closely linked to its nucleotide status (Modica et al., 2017). The GTP-bound active Rab7 is associated to membranes, while upon hydrolysis the GDP-bound Rab7 is inactive and recycles to the cytosol. This switch is stimulated by guanine nucleotide exchange factors (GEFs) and GTPase-activating proteins (GAPs) (Müller and Goody, 2018). Also other reversible post-translational modifications (PTMs) like phosphorylation, palmitoylation, and ubiquitination modulate the ability of Rab7 to orchestrate membrane trafficking at LEs (Modica and Lefrancois, 2020; Modica et al., 2017).

On a cellular level, Rab7 has several important roles in the late endocytic pathway. Figure 1-3 highlights four of them:

(I) During maturation, the conversion from Rab5 to Rab7 is promoted by a feedback loop of Rab7 activation through Rab5 and subsequent Rab5 suppression (Rink et al., 2005).

(II) The effector RILP (Rab7-interacting lysosomal protein) binds downstream complexes like HOPS (homotypic fusion and protein sorting) for vesicle tethering and fusion (van der Kant et al., 2013).

(III) RILP and another effector FYCO1 (FYVE and coiled-coil domain containing protein 1) mediate attachment of late endosomes to dynein and kinesin motors on microtubules for endosome and lysosome motility (Jordens et al., 2001; Pankiv et al., 2010).

(IV) Interaction of Rab7 and the retromer cargo recognition complex is required for recycling of transmembrane cargo like Cl-M6PR from endosomes back to the TGN (Rojas et al., 2008).

Similar to its functions on endosomes, Rab7 is important in docking, fusion, transport, and clustering of autophagosomes during the entire autophagy process (Gutierrez et al., 2004; Jäger et al., 2004). 


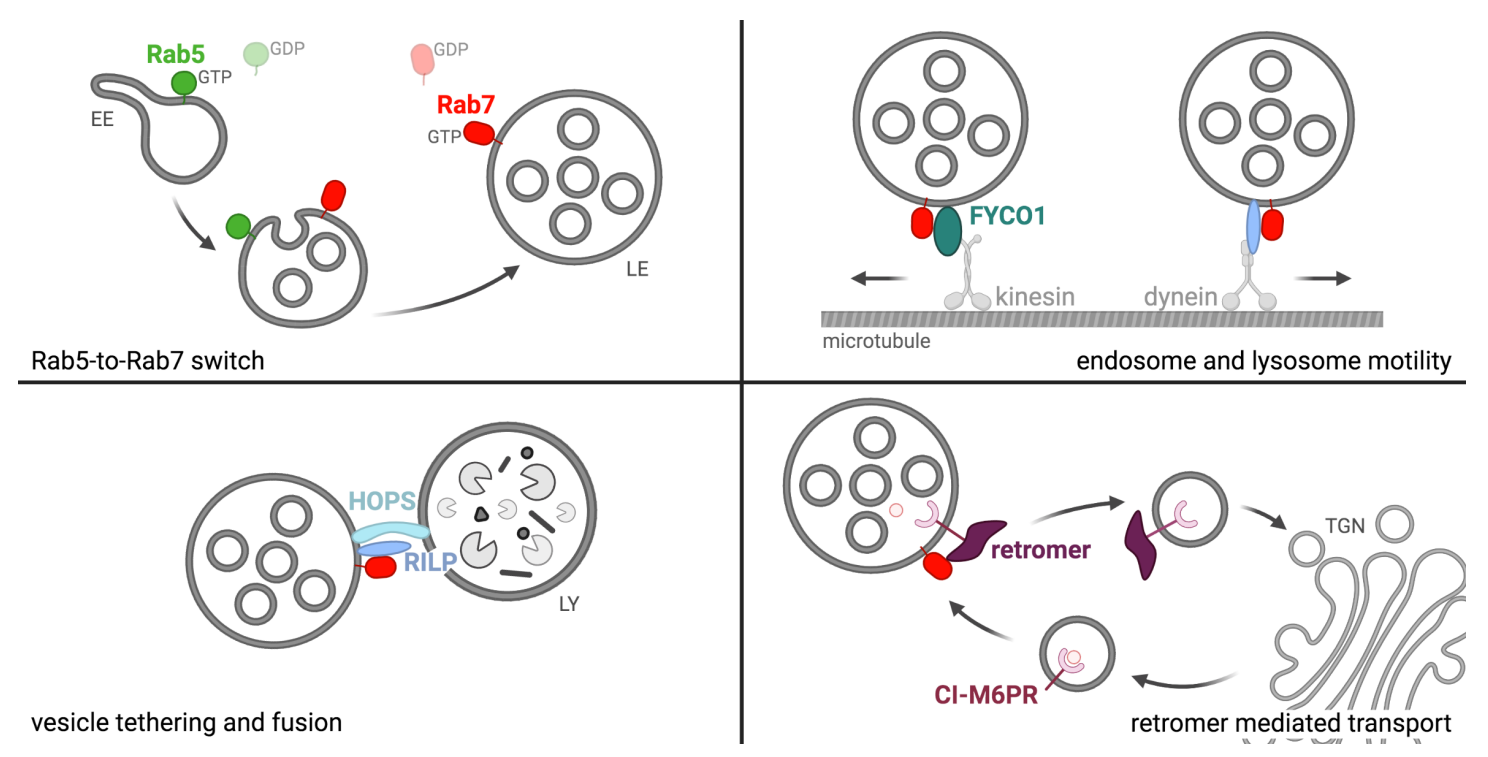

Figure 1-3: Rab7 functions During endosome maturation from early (EE) to late endosomes (LE) Rab7 coordinates the Rab5-to-Rab7 switch. With the help of its effector protein RILP, Rab7 mediates the tethering and fusion of endosomal vesicles like late endosomes and lysosomes. Rab7 interaction with effectors RILP and FYCO1 enables kinesin- and dynein-dependent transport of endosomes along microtubules. Rab7 is involved in the retromer mediated recycling of $\mathrm{Cl-M6PR}$ to the TGN.

\subsection{Lysosomes}

Lysosomes represent the end point of the degradation pathway with several feeder pathways. Besides from the endocytic and secretory pathway, lysosomes receive and degrade macromolecules from autophagy and phagocytosis (Luzio et al., 2007). Degradation in the lysosomal lumen is catalyzed by more than 60 hydrolases. These hydrolases are synthesized, tagged with a mannose-6-phosphate, and recognized by mannose-6-phosphate receptors at the TGN. After transport to endosomes, hydrolases dissociate from their receptors due to the low $\mathrm{pH}$. This allows recycling of the empty receptors back to the TGN and further transport of the hydrolases to the lysosomes via late endosomes (Saftig and Klumperman, 2009; Seaman, 2004). Unlike soluble hydrolases, membrane proteins are delivered from the TGN to the lysosomes either by a direct intracellular route or an indirect route via the plasma membrane (Bonifacino, 2004; Braulke and Bonifacino, 2009).

Though lysosomes cluster in the perinuclear region, they are motile and move bidirectionally along microtubule tracks. Several interactions with different factors, like the endoplasmic reticulum and Golgi, actin filaments, or microtubule motors, contribute to these dynamics. Lysosome positioning and motility is predominantly regulated by nutrient availability leading to redistribution of the organelle towards the perinuclear region upon removal of amino acids (Korolchuk et al., 2011; Pu et al., 2016).

Lysosomes do not only have an essential role in maintaining cellular homeostasis, but are a dynamic signaling hub. The "degradation organelle" senses and controls the metabolic status and signaling, mediates transcription programs like lysosome biogenesis and autophagy, and communicates with other cellular structures via membrane contact sites (Ballabio and Bonifacino, 2020). 


\subsection{1 mTORC1 signaling at lysosomes}

By maintaining cellular catabolism and sensing the nutrition status of the cells, lysosomes can inform the cell of the intra- and extracellular milieu and adapt to those conditions. The everimportant key regulator of cellular biosynthetic pathways mTORC1 (mechanistic target of rapamycin complex 1) dynamically associates with lysosomes (Sancak et al., 2010). In presence of nutrients and amino acids, active mTORC1 phosphorylates numerous effectors to support cell anabolism and growth, while inhibiting catabolic pathways like autophagy (Saxton and Sabatini, 2017; Yu et al., 2010). Regulation of mTORC1 activity is a complex multi-step process where a series of signaling events converge. Amino acid-dependent mTORC1 translocation to the lysosomal surface is mediated by the heterodimeric small GTPases Rag A/B-C/D (Jewell et al., 2013; Kim et al., 2008). The Rags, as anchor for mTORC1 on lysosomes, are regulated by the Ragulator complex which coordinates the Rag GTP loading with its GEF activity in response to amino acid availability. Ragulator GEF activity is regulated by amino acids via its interaction with the lysosomal v-ATPase and the nutrient sensor SLC38A9. Also SLC38A9 shows GEF activity towards the Rags (Rebsamen et al., 2015; Shen and Sabatini, 2018; Zoncu et al., 2011). Under nutrient rich conditions, the Rag heterodimer is in its active RagA/B ${ }^{G T P}-$ RagC/D ${ }^{G D P}$ form and recruits $m$ TORC1, whereas under starvation RagA/ $B^{G D P}-R a g C / D^{G T P}$ is inactive and shows low affinity for mTORC1 (Bar-Peled et al., 2012). Ragulator is a pentameric protein complex consisting of a membrane anchored LAMTOR1 and two roadblock heterodimers LAMTOR2-3 and LAMTOR4-5. Structural analyses of the Ragulator-Rag-mTORC1 complex reveal that: (I) Ragulator is anchored to the lysosomal membrane via the $\mathrm{N}$-terminal myristoylation and palmitoylation of LAMTOR1 (Nada et al., 2009). (II) LAMTOR1 is essential for the assembly of the components since it wraps around the LAMTOR2-5 heterodimers. In addition, it coordinates and stabilizes the binding of the Rags. (III) The Rag heterodimer interacts with Ragulator through their C-terminal domains. (IV) mTORC1 is recruited through the interaction of its Raptor subunit with Rags (de Araujo et al., 2017; Yonehara et al., 2017). After localization of mTORC1 to lysosomes via Rag GTPases, the active GTPase RHEB GTP (Ras homolog enriched in brain) upregulates mTORC1 kinase activity by its interaction with the mTOR subunit (Long et al., 2005).

Though recent biochemical and structural studies have shed light on the molecular basis of amino acid-dependent $\mathrm{mTORC} 1$ regulation, there are still unknown regulatory mechanisms. 


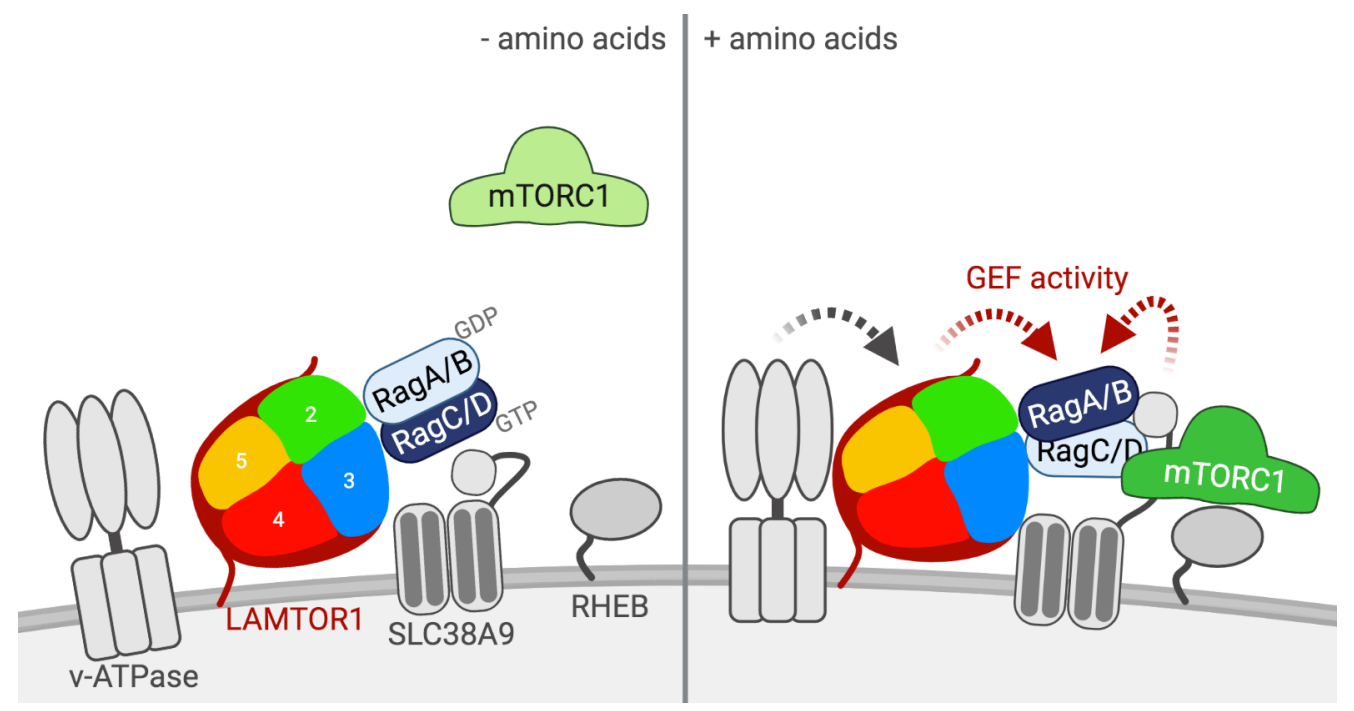

Figure 1-4: mTORC1 acitvation at the lysosomal surface Under amino acid deprived conditions the Rag GTPases remain in their inactive form and show low affinity for mTORC1. Inactive mTORC1 resides in the cytoplasm. High amino acid levels are sensed by the lysosomal V-ATPase and SLC38A9. In turn, Ragulator (consisting of LAMTOR1-5) and SLC38A9 GEF activities are stimulated. The nucleotide switch activates RagA/B-RabC/D. mTORC1 is recruited to the lysosomal surface where it is activated by RHEB.

\subsubsection{Transcriptional regulation of mTORC1 via TFEB}

One lysosomal adaption to cellular stresses like starvation of amino acids is the transcriptional regulation of genes of the endosomal-lysosomal system. Active mTORC1 directly mediates phosphorylation of transcription factor EB (TFEB). Its subcellular localization is nutrientdependent, with TFEB translocating to the nucleus upon starvation. TFEB is the master transcriptional regulator driving expression of autophagy and lysosomal genes (Settembre et al., 2011). An mTORC1-TFEB feedback loop has an important role in this adaption program. Starvation induced TFEB activation drives transcription of Rags. In turn, mTORC1 is activated again and inhibits TFEB through phosphorylation (Di Malta et al., 2017).

\subsubsection{Initiation of autophagy by mTORC1}

Upon nutrient deprivation and mTORC1 suppression, macroautophagy (hereafter autophagy) is one of the key outcomes to maintain cellular homeostasis. Autophagy proceeds through well defined and continuous maturation steps: (I) induction, (II) membrane nucleation, (III) autophagosome elongation, and (IV) autophagosome maturation and fusion with the lysosome (Mizushima, 2007).

mTORC1 is a master regulator of autophagy, which was first shown to inhibit the induction steps but furthermore is involved much beyond the initial step. Direct substrates of mTORC1 determine the function of all steps of autophagy with following examples (Dossou and Basu, 2019; Saxton and Sabatini, 2017): (I) induction - ULK1 (Shang et al., 2011), (II) nucleation AMBRA1 (Nazio et al., 2013), (III) elongation - WIPI2 (Wan et al., 2018), (IV) maturation and fusion - UVRAG (Kim et al., 2015).

As described before, autophagy is not only regulated by phosphorylation via mTORC1, but also on a transcriptional level. TFEB was reported to increase the expression of autophagy genes like UVRAG, WIPI2, LC3, p62, and more (Sardiello et al., 2009; Settembre et al., 2011). 
Notably, autophagosomes fuse with lysosomes and deliver cargo to the late endocytic pathway. Thus, autophagic processes also account for the complex nature of multivesicular bodies and lysosomes.

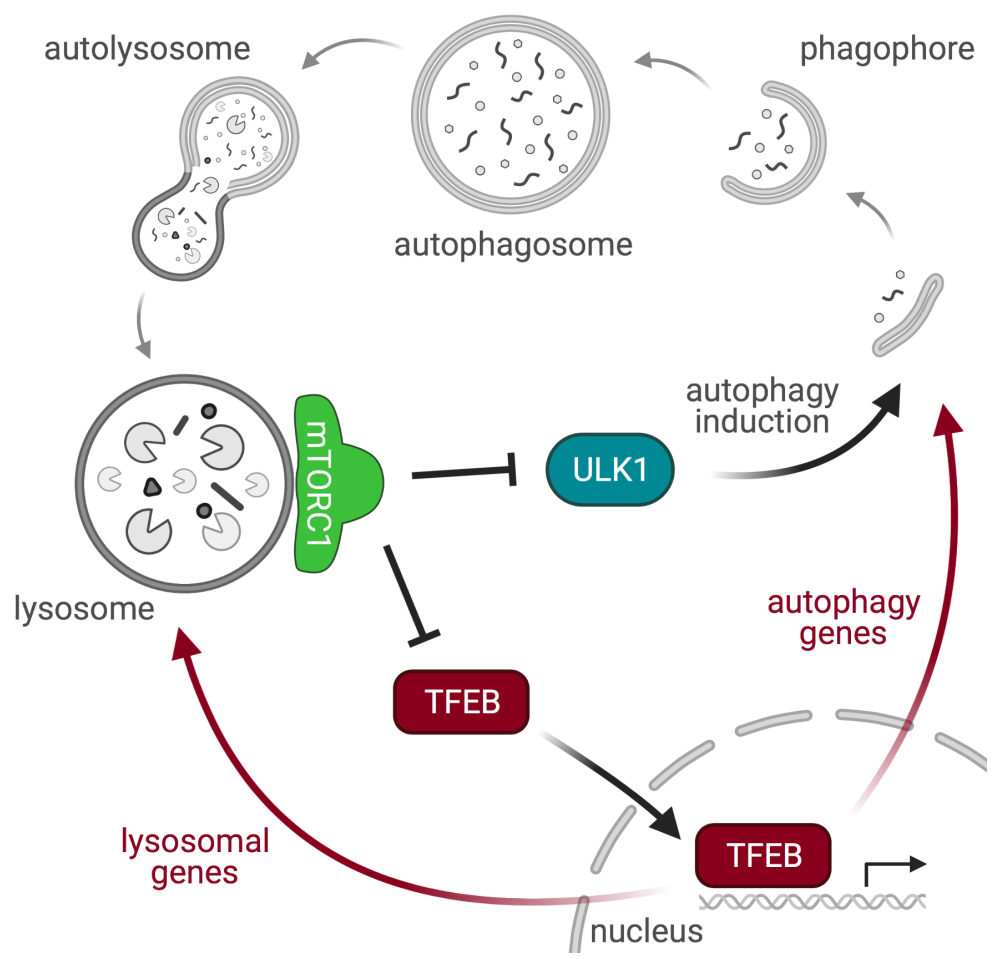

Figure 1-5: mTORC1 regulation of transcription and autophagy Active mTORC1 inhibits transcription and autophagy by phosphorylating TFEB and ULK1. mTORC1 suppression mediates TFEB translocation to the nucleus and transcription of lysosomal and autophagy genes. Furthermore, additional activation of ULK1 induces autophagy.

\subsection{The ubiquitin system}

Post-translational modifications control and specify the majority of the proteome in a cell. One of the most prominent modifiers is the evolutionary conserved small protein ubiquitin. Ubiquitination, the covalent ligation of ubiquitin molecules to substrates, was originally identified as a proteasomal degradation signal, but is now known to serve also protein interactions, activity, localization, and more non-proteolytic events. Overall, the versatile ubiquitin modification is involved in a wide variety of cellular pathways like cell division, intercellular communication, and intracellular signaling and is implicated in pathophysiological states and diseases. The importance and complexity of the system requires a tight regulation by a network of proteins to write, read, edit, or erase the ubiquitin signal (Clague et al., 2015; Grabbe et al., 2011).

\subsubsection{Ubiquitination of substrates}

Ubiquitin consists of 76-amino acid protein and is ubiquitously expressed in eukaryotes. It can be covalently attached to lysines (and to a minority other residues) of substrates via its Cterminal glycine through an enzymatic cascade. The inactive ubiquitin precursor undergoes proteolytic progression to reveal its C-terminal di-glycine motif Gly75-Gly76, which can be used 
to form an isopeptide bond with the substrate's lysines (Hershko and Ciechanover, 1998; Pickart and Rose, 1985; Scheffner et al., 1995).

The subsequent three-step process includes E1 activating enzymes, E2 conjugating enzymes, and E3 ligases, the writers of the ubiquitin code. First, E1 enzymes activate ubiquitin in an ATPdependent manner by the formation of C-terminal high-energy thioester bond between the Cterminal carboxylate of ubiquitin and E1 catalytic cysteine. In humans, two E1 enzymes are identified to activate ubiquitin for the entire array of downstream conjugating enzymes (Schulman and Harper, 2009). Concomitant structural rearrangements increase the affinity for E2 enzymes allowing the next step in the conjugating cascade. Ubiquitin is transferred from the E1 cysteine to the E2 active site cysteine and forms a new thioester bond. The E1 enzyme is subsequently released. There are 35 E2 family members in the human genome, which dictate to ubiquitin conjugation (van Wijk and Timmers, 2010). In the last step, the E3 ligases catalyze the linkage of ubiquitin via its C-terminal glycine onto a lysine of the substrate. This transfer can be either directly from E2 to the substrate (mediated by RING/U-box ligases performing a scaffolding function), or indirectly by formation of an ubiquitin-E3 intermediate (for HECT and RBR ligases) (Clague et al., 2015; Pickart and Rose, 1985). Over 600 genes have been predicted to be members of the E3 ligase families (Zheng and Shabek, 2017). In terms of gene numbers, the cascade has pyramidal organization with $2: 35:>600$ (E1:E2:E3). But the total protein copy numbers present an estimated ratio of 1:3:2 (E1:E2:E3) in HeLa cells (Kulak et al., 2014).

Substrates cannot only be monoubiquitinated on one or more sites, but they can also be sequentially modified with polyubiquitin chains. Ubiquitin contains seven lysine residues (K6, $\mathrm{K} 11, \mathrm{~K} 27, \mathrm{~K} 29, \mathrm{~K} 33, \mathrm{~K} 48$, and $\mathrm{K} 63$ ) that can be used for the transfer of other ubiquitin molecules. This allows the formation of homo-, heterotypic, and branched chains. Furthermore, ubiquitin can also form linear chains via the conjugation to the $\mathrm{N}$-terminal methionine residue (Met1) of another ubiquitin molecule (Kirisako et al., 2006). Adding another layer of complexity, unanchored ubiquitin chains in cells are characterized as active members of the ubiquitin code. All linkages types contribute to the generation of diverse ubiquitin signaling in cells. Chain linkage type and substrate specificity are achieved by the E2 or E3 enzymes during the assembling cascade (Akutsu et al., 2016; Blount et al., 2020; Yau and Rape, 2016).

The diverse information that is encoded by the ubiquitin signal is recognized and translated by the readers of the code. For this, a range of specific proteins contains ubiquitin binding domains with distinct chemical and structural features (Rahighi and Dikic, 2012). 


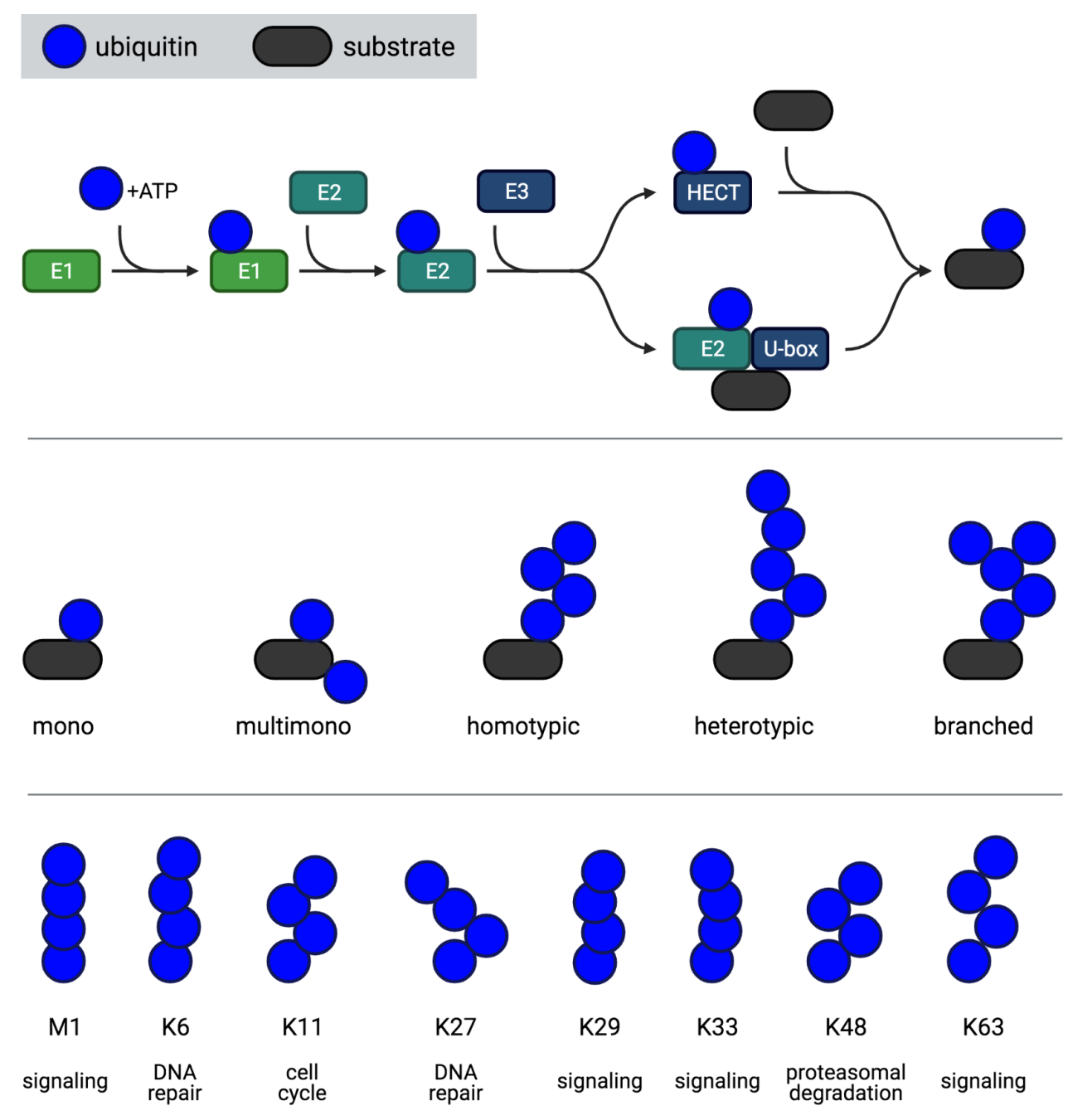

Figure 1-6: Overview of substrate ubiquitination Ubiquitin is conjugated to substrates in a three-step enzymatic cascade involving E1 ubiquitin activation, E2 ubiquitin conjugation, and direct or indirect E3 ubiquitin ligation. A substrate can be decorated with one ubiquitin on one or several residues, or different chains can be formed. Ubiquitin chains encode diverse outcomes depending on their linkage type (Akutsu et al., 2016).

\subsubsection{Deubiquitinating enzymes}

Ubiquitination is a reversible process. The editing and erasing of the signal are mediated by deubiquitinating enzymes (DUBs), which hydrolyze the ubiquitin linkages. Doing so, DUBs process ubiquitin precursors, maintain the levels of free ubiquitin levels and control the dynamics of ubiquitin signaling events (Komander et al., 2009). The human genome encodes around 100 DUBs in seven major classes differentiated by their catalytic mechanism. Six of the seven DUB families are cysteine proteases: ubiquitin specific proteases (USPS), ubiquitin Cterminal hydrolases (UCHs), ovarian tumor proteases (OTUs), Machado-Josephin proteases (MJDs), the motif interacting with ubiquitin (MIU)-containing DUB family (MINDYs), and the latest addition, the Zinc finger-containing ubiquitin peptidase 1 (ZUFSP/ZUP1). The JAB1/MPN/MOV34 proteases (JAMMs) are the only DUBs that are zinc metalloproteases (Abdul Rehman et al., 2016; Komander et al., 2009; Kwasna et al., 2018). 


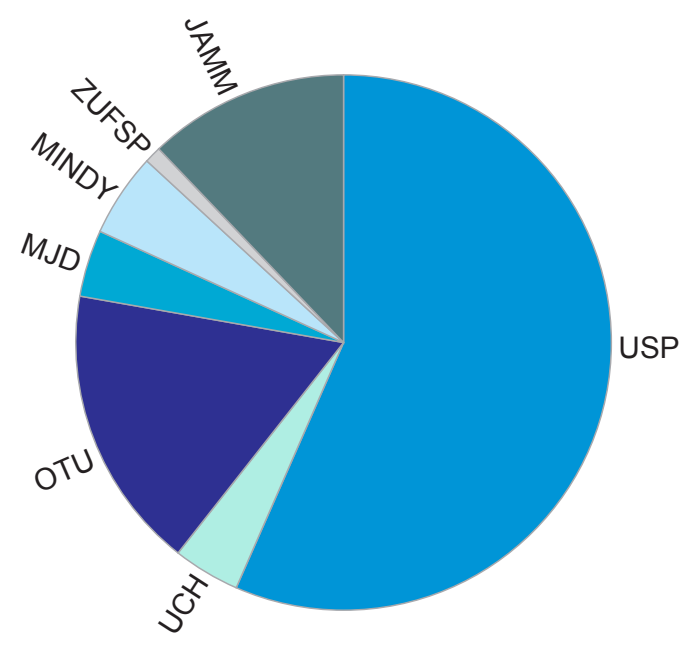

Figure 1-7: DUB families Schematic overview of the seven DUB families and their number of members. USPS are the biggest family (56 members), followed by OTUs (17), JAMMs (12), MINDYs (5), UCHs (4), MJDs (4), and ZUFSP (Clague et al., 2019).

DUB enzymatic activities can show specificity for the chain linkage or the substrate. Aside from the chain linkage type, a DUB can preferentially cleave an ubiquitin moiety at a certain position within the chain (endo- or exo-deubiquitination), a monoubiquitin or the entire chain from a substrate. Linkage specificity requires the recognition and binding of two specifically linked ubiquitins across their active site. Such activity is displayed by the OTU family DUBs with their chain linkage over substrate preferences (Mevissen et al., 2013). By contrast, USPs show low chain type specificity, but interact selectively with their substrates via additional protein-protein interaction domains (Clague et al., 2013; Faesen et al., 2011).

With their essential role in the regulation of ubiquitin-dependent processes, malfunction of DUBs can result in dramatic cellular and physiological consequences. Thus, a tight regulation is crucial for proper function and fine-tuning of deubiquitinating enzymes. Multiple ways are described to manage their abundance, localization, and catalytic activity involving additional interwoven layers of post-translational processing, modification, and binding of proteins or molecules (Sahtoe and Sixma, 2015). 


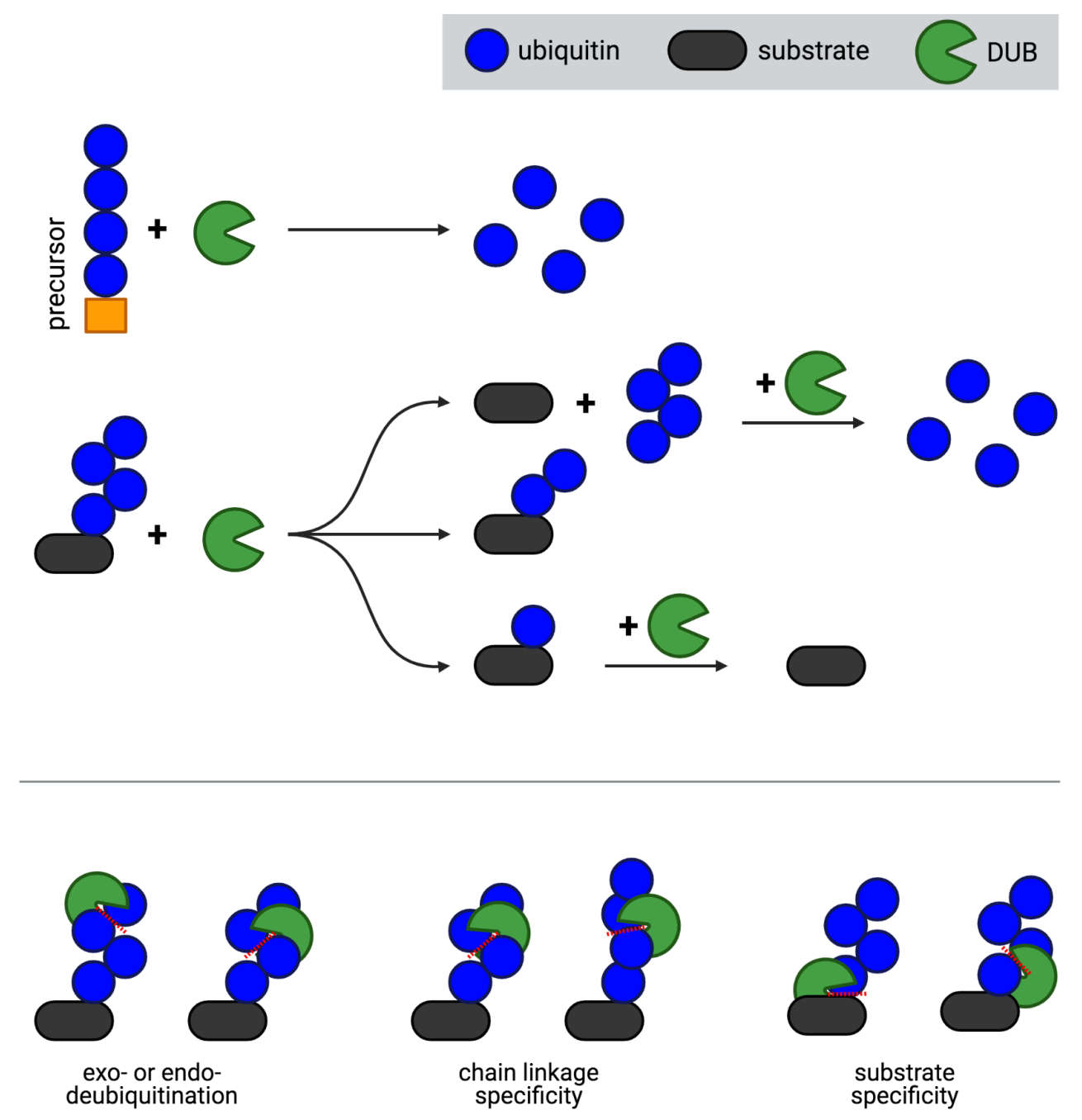

Figure 1-8: Overview of deubiquitination Deubiquitinating enzymes (DUBs) have major roles in the processing of ubiquitin precursors, and modulating or terminating the ubiquitin signal on a substrate. DUBs cleave entire ubiquitin chains from a substrate and disassemble the chain into single moieties. Furthermore, the signal can be edited by truncation to shorter chains or leaving the proximal ubiquitin on the substrate. Additionally, DUBs show different specificities for the position within in the chain, the chain linkage or the substrate.

\subsubsection{Ubiquitin regulation of the endosomal-lysosomal system}

Ubiquitination generates a complex signaling code balanced by the opposing action of DUBs. They serve a plethora of cellular function and are important for cellular activity. One of the highly regulated processes, in which reversible ubiquitination has an impact, is the endosomal sorting and membrane trafficking (Clague and Urbé, 2017).

Ubiquitination can mediate internalization of activated receptors. Adaptor proteins couple to these modified receptors for both clathrin-dependent and -independent endocytosis. The first and best-known ubiquitinated plasma membrane receptor in human is the epidermal growth factor receptor (EGFR). EGFR is ubiquitinated upon ligand activation by the E3 cCbl leading to its internalization in clathrin-coated pits (Goh et al., 2010; Levkowitz et al., 1999). During the further course, the modification is used to sort EGFR in MVBs towards the lysosomal pathway using the ESCRT machinery (Clague et al., 2012; Huang et al., 2006). More than 15 DUBs were identified to impair EGFR turnover, including AMSH (knockdown initiates enhanced degradation, McCullough et al., 2004), USP8 (indirect regulation by controlling ESCRT 
proteins, Row et al., 2006), and USP9X (targeting of endocytic adaptor Eps15, Savio et al., 2016).

Another example of ubiquitin and DUB regulation represents the WASH complex (member of the Wiscott-Aldrich syndrome proteins) and its participation in the retromer mediated recycling by recruitment of F-actin for membrane tubule scission. WASH is positively regulated by ubiquitination through the E3 MAGE-L2-TRIM27. In this context, USP7 deubiquitinates WASH, but also, controversially, stabilizes TRIM27 (Hao et al., 2013; Hao et al., 2015).

Ubiquitin also plays a role in the later endocytic pathway at lysosomes. Lysosomal proteins like LAMP1 and LAMP2 have been shown to be ubiquitinated upon lysosomal damage (Yoshida et al., 2017). Only recently, the E2 enzyme UBE2QL1 was described to be critical for maintaining lysosome integrity and lysophagy, the removal of damaged lysosomes by autophagy (Koerver et al., 2019). Furthermore, also the DUB YOD1 is required for the clearance of damaged lysosomes during the endo-lysosomal damage response (Papadopoulos et al., 2017).

\subsection{The deubiquitinating enzyme USP32}

The ubiquitin specific protease 32 (USP32) is a highly conserved but rather uncharacterized DUB. Its sequence contains 1604 amino acids and, with a predicted molecular weight of $182 \mathrm{kDa}$, is one of the bigger DUBs. USP32 has, like all other USPs, a catalytic USP domain showing catalytic activity but no chain linkage preference (Akhavantabasi et al., 2010; Sapmaz et al., 2019). As already mentioned earlier, USP DUBs often contain other domains and shorter structural motifs, which may regulate activity and govern interactions (Clague et al., 2013). Ubiquitin like (UBL) domains can regulate the catalytic activity of USPs. USP32 and other USPS contain a domain present in ubiquitin specific proteases (DUSP), which is proposed to play a role in protein-protein interaction or substrate recognition (Clague et al., 2013; de Jong et al., 2006). Strikingly, USP32 has two domains that are unique in the whole DUB family. The EF hands, calcium-binding motifs, at its $\mathrm{N}$-terminus function generally in protein regulation and interaction (Lewit-Bentley and Réty, 2000). The second USP32-exclusive domain is a CAAX box for possible prenylation at its $\mathrm{C}$-terminus. Protein prenylation is an irreversible post-translational lipid modification where either farnesyl (15-carbon) or geranylgeranyl (20-carbon) isoprenoids are added to the CAAX box at the $\mathrm{C}$-terminus of intracellular proteins. This process is critical for proper function of many proteins, particularly for anchoring the proteins to membranes (Gao et al., 2009).

The two DUBs USP32 and USP6 originate from the same gene progenitor. They share $98 \%$ identity of their USP domains, but USP32 lacks the TBC domain present in USP6 (Paulding et al., 2003). Despite their similarity and the reported function of USP6 as an oncogene and its involvement in endocytic trafficking (Madan et al., 2016; Martinu et al., 2004; Paulding et al., 2003), the physiological and cellular function of USP32 is poorly characterized. A few studies suggest its oncogenic role in breast, small cell lung, and gastric cancer (Akhavantabasi et al., 2010; Dou et al., 2020; Hu et al., 2017). On a cellular level, USP32 fused to green fluorescent protein (GFP) was reported to localize to the Golgi and VPS35, the retromer CRC component, was identified as a high-confidence candidate interacting protein (Akhavantabasi et al., 2010; 
Sowa et al., 2009). Furthermore, during the course of this study, Rab7 was described as a potential substrate of USP32 (Sapmaz et al., 2019).

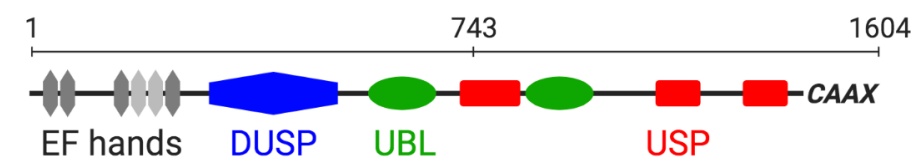

Figure 1-9: Domain structure of USP32 USP32 features N-terminal EF-hands for possible calcium regulation, DUSP and UBL for regulation of interactions and catalytic activity, catalytic active USP with the catalytic cysteine at position 743, and C-terminal CAAX box for possible prenylation. 


\section{Aims}

Ubiquitination of proteins functions as a versatile signal in most cellular pathways. Deubiquitinating enzymes (DUBs) release conjugated ubiquitin from proteins and thereby control the precise outcome of ubiquitin signals. While the cellular and physiological role of many DUBs has already been identified, there are still various DUBs whose cellular function remains elusive. The ubiquitin specific protease 32 (USP32) is one of those poorly characterized enzymes. Only few publications linked the DUB to different types of cancer (Akhavantabasi et al., 2010; Dou et al., 2020; Hu et al., 2017). Furthermore, the localization of overexpressed GFPUSP32 at the trans-Golgi network and the reported interaction with VPS35, the core component of the retromer cargo recognition complex, suggested a role for USP32 in the endosomal system (Akhavantabasi et al., 2010; Sowa et al., 2009).

A small number of DUBs was already shown to regulate the endosomal and lysosomal pathways at various stages such as receptor internalization and recycling from and to the plasma membrane, protein sorting and membrane trafficking in endosomal vesicles, as well as response to lysosomal damage (Clague and Urbé, 2017; Hao et al., 2015; Papadopoulos et al., 2017).

The present study aimed to characterize the cellular role of USP32 with a focus on the endosomal-lysosomal system. Therefore the following objectives were defined:

(I) Establishing tools to investigate the physiological function of USP32 in a normal cellular model (no disease background)

(II) Basic characterization of the cellular abundance and localization of endogenous USP32

(III) Identification of USP32 substrates in an unbiased approach

(IV) Characterization of USP32 substrates including the validation of their USP32-regulated ubiquitination and investigation of the cellular functions of these ubiquitin signals 


\section{$3 \quad$ Results}

\subsection{Abundance of USP32 in different cell lines}

To investigate the cellular function of USP32, tools for cell culture based experiments were established. The abundance and molecular weight of endogenous USP32 (theoretically calculated $182 \mathrm{kDa}$ ) was shown in different cell types, namely the primary epithelial cell line hTERT RPE1 (hereafter RPE1), U2OS osteosarcoma cells, HEK293 embryonal kidney cells, and MCF7 breast adenocarcinoma cells. For short-term depletion of the DUB, transient transfection of small interfering RNA (siRNA) for gene silencing was used. USP32 depletion with one sequence-specific siRNA (siUSP32) was detected by immunoblotting. It could be shown that a specific antibody detects USP32 at $180 \mathrm{kDa}$, and protein levels, according to literature, and siRNA efficacy depend on the cell line (Figure 3-1 A).

Gene silencing using RNA interference only transiently depletes USP32. In addition, transfection efficiency is dependent on the cell line and the procedure could produce stress already. Therefore it was necessary to establish constitutive USP32 knockout (KO) cells with the CRISPR/Cas9 technology in collaboration with Verena Bittl (AG Bremm, Institute of Biochemistry II, Goethe University Frankfurt). In order to study USP32 function without any disease background and due to their respective specific characteristics for different technical approaches, RPE1 and U2OS cells were used to generate USP32KO cell lines. Three different guide RNAs specific for USP32 as well as for non-human targeting (NHT) sequences for control were designed (see 6.2.1.7). For technical reasons, only RPE1 NHT cells were established, and parental wildtype (wt) cells were used for control (ctrl) in U2OS cells. USP32 knockout was verified by immunoblotting using a USP32 specific antibody (Figure 3-1 B). USP32 was efficiently knocked out in RPE1 and U2OS cells and these cells were used as polyclonal cell lines for further experiments. 


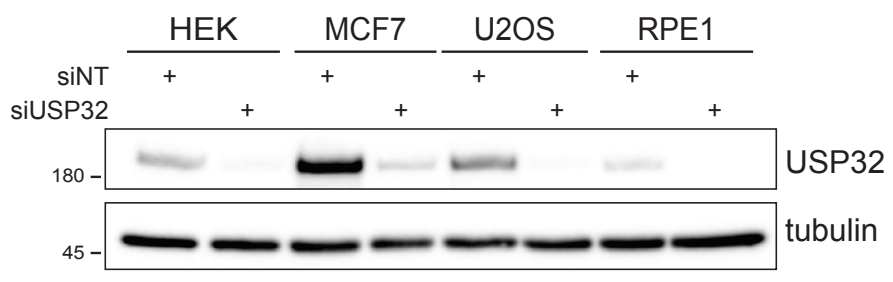

B

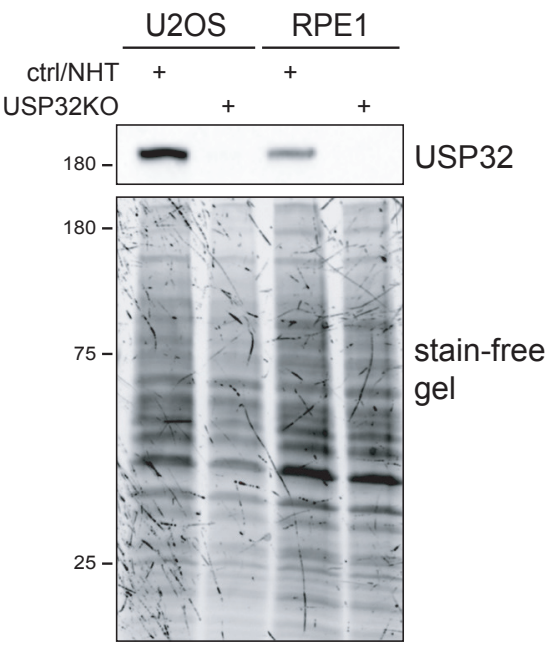

Figure 3-1: Expression levels and depletion of USP32 in different cell types. A Expression levels of USP32 vary in different cell types. HEK293, MCF7, U2OS, and RPE1 cells were transfected with either non-targeting control SiRNA (siNT) or siRNA targeting USP32 (siUSP32). USP32 protein levels were detected by Western blotting. B U2OS and RPE1 USP32 knockout (KO) cells were generated using the CRISPR/Cas9 technology. USP32 protein levels were detected by Western blotting confirming the knockout.

\subsection{Expression of exogenous USP32}

In order to study USP32 function and regulation, plasmid DNA for transient transfection of different USP32 constructs would be useful as a tool for cell culture based experiments. Based on the predicted domain structure of USP32, truncated versions lacking different domains were designed (Figure 3-2 A). The open reading frames (ORFs) were amplified by PCR and subcloned into different plasmid backbones for mammalian expression to express tagged versions of USP32.

First, full length and truncated USP32 was successfully cloned into pEGFPN1 for C-terminal tagging. None of the constructs expressed in HEK293 cells. Since the position of a tag can influence the expression of a protein, next the position of the GFP tag relative to USP32 was swapped to the N-terminus by subcloning the wildtype full length version into pEGFPC1. The expression was tested in several experiments under different conditions. Regardless which condition was tested (Table 3-1), the produced protein was detected on a Western blot with a GFP antibody always at $\sim 135 \mathrm{kDa}$, meaning $70 \mathrm{kDa}$ less than expected.

Table 3-1: Tested conditions for USP32 expression

Cell line

Transfected DNA amount

Transfection reagent

Time before lysis

Lysis buffer

treatment

Co-transfection with 14-3-3 protein YWHAB
HEK293, U2OS, MCF7, RPE1

0.5-2 $\mu \mathrm{g}$

PEl, GeneJuice

$24 \mathrm{~h}, 48 \mathrm{~h}$

RIPA, 2xLDS

BafA1, MG132

$0.5-2 \mu \mathrm{g}$ 
At this stage of the project, two publications presented data with overexpressed USP32 in cell culture experiments indicating that the correct expression itself was possible (Akhavantabasi et al., 2010; Funakoshi et al., 2014). Sylvie Urbé (Institute of Translational Medicine, University of Liverpool) provided a new expression construct containing a longer linker region between GFP tag and USP32. Based on this construct, all truncated USP32 versions as well as the full length (fl) USP32 were generated again. A test expression of USP32 $\mathrm{fl}$ and N-terminal truncated constructs 362/732-1604 in HEK293 cells resulted again in an apparently shorter protein on a GFP immunoblot (Figure 3-2 B). Additional bands on the blot showed the same pattern for all samples.

GFP has $\sim 27 \mathrm{kDa}$ and is a rather big tag, which could negatively affect expression of a protein. Therefore, the USP32 $\mathrm{fl}$ and 362-1604 ORF was subcloned in a pIRES-HA-C1 backbone to have a small C-terminal HA tag. Test expression of pIRES-HA-USP32 $\mathrm{fl}$ in HEK293 cells and immunoblotting with a HA and USP32 specific antibody resulted in bands at following molecular weights (Figure 3-2 C).

A

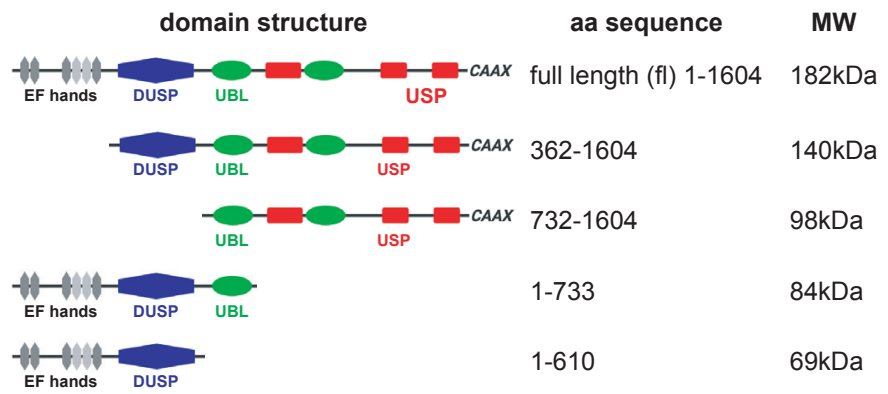

B

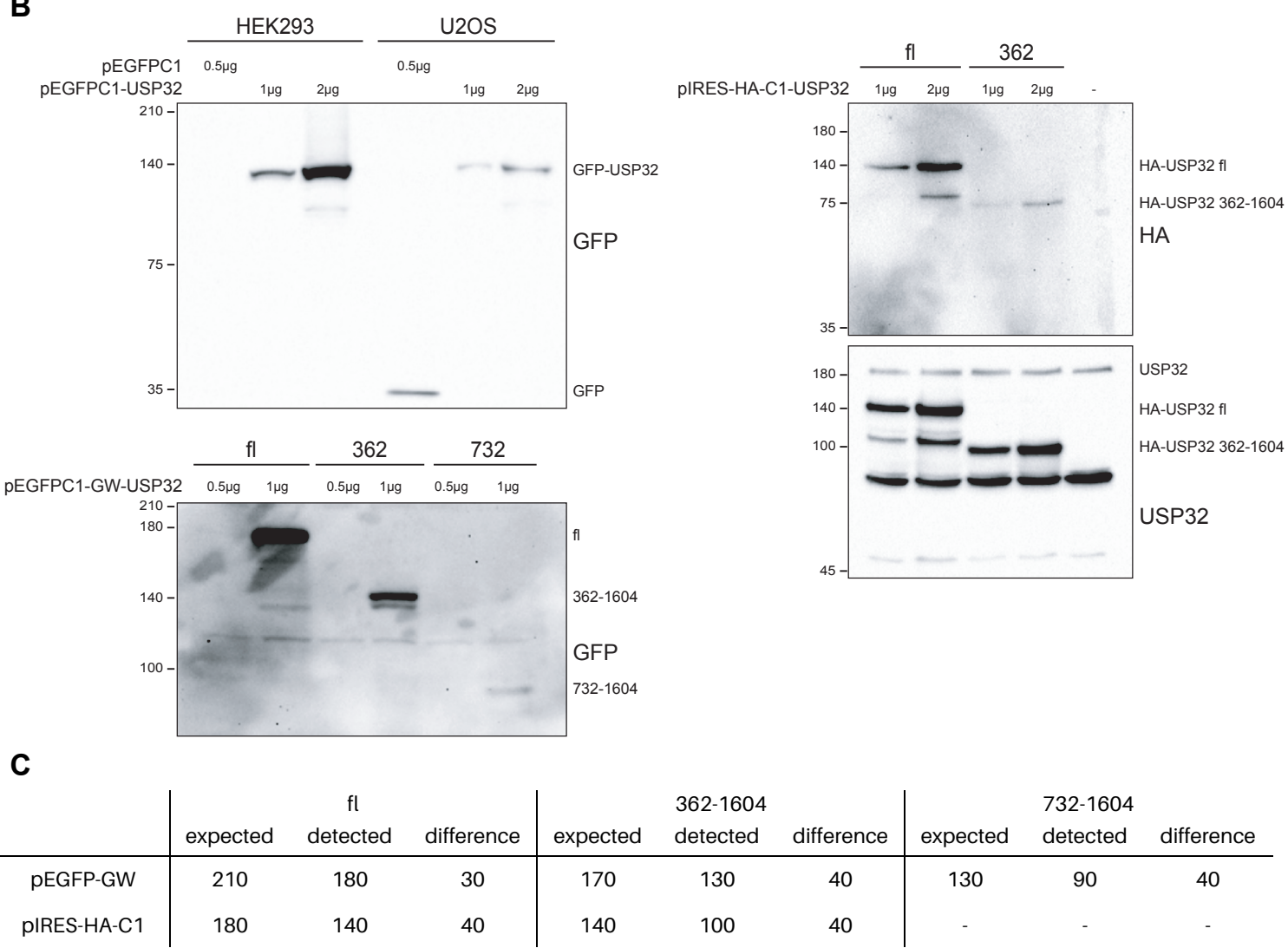


Figure 3-2: Different USP32 constructs were designed and expressed in cells. A USP32 was N- or $\mathrm{C}$-terminally truncated. The cartoon shows the domain structures, amino acid sequence and molecular weight (MW) of designed constructs. B Varying amounts of GFP- or HA tagged USP32 were transiently transfected in HEK293 or U2OS cells and cell lysates were analyzed by immunoblotting. C Overview of detected molecular weights of exogenous USP32 in comparison to the theoretically calculated

Furthermore, a catalytic inactive USP32 version was generated by site-directed mutagenesis of the catalytic cysteine at position 743 to a serine. The mutation to a serine was chosen to avoid possible substrate trapping effects of a cysteine-to-alanine or -arginine mutant (Morrow et al., 2018).

The attempted measures to create a tool for cellular expression of USP32 can be recapitulated as follow: (I) C-terminal tagging of USP32 abolished expression in cell culture at all. (II) C-terminal tagging in pEGFPC1 resulted in expression of a construct with detected size of $\sim 70 \mathrm{kDa}$ less than theoretically calculated, regardless of the additional tested condition. (III) Cloning a longer linker between USP32 and GFP as well as cloning the small HA tag both at the N-terminus of USP32 $\mathrm{fl}$ and truncated versions produced an expressed protein with apparent size of $\sim 35 \mathrm{kDa}$ smaller than expected.

Sanger sequencing verified the sequences of all DNA constructs to be correct. Since the smaller USP32 constructs could be detected with the antibodies specific for the tag, a cleavage from the $\mathrm{C}$-terminus could be possible. But a potential degradation or cleavage site in the sequence would be at the same position regardless of the truncation. Thus, the detected part would have the same size and the "missing part" would vary in size. However, the detected missing part had always $\sim 35 \mathrm{kDa}$. Eventually, it was assumed that the produced USP32 was correct but showed unexpected running behavior on a SDS page due to the tagging.

For this project, it was decided to execute the majority of experiments with CRISPR KO cells and to investigate the effect of USP32 depletion. Overexpression of tagged USP32 was performed only when absolutely necessary with either a GFP or HA tag.

\subsection{Cellular localization of USP32}

The predicted domain structure of USP32 includes a prenylation site for possible lipidation and membrane insertion at its C-terminus. To investigate the localization of USP32, a subcellular fractionation of RPE1, U2OS and MCF7 cells was performed and analyzed by immunoblotting. USP32 was found to be predominantly in the cytoplasmic fraction but also partly in the membrane fraction of all tested cell lines (Figure 3-3 A).

The used antibody against USP32 showed only specific immunofluorescence (IF) staining in MCF7 cells with already elevated protein levels. Immunofluorescence co-staining of USP32 together with the cis-Golgi marker GM130 and the lysosomal marker LAMP1 in MCF7 cells showed partially overlap of these proteins (Figure 3-3 B). The subcellular fractionation and immunofluorescence staining suggested that USP32 was localized in the perinuclear region of cells, at membrane organelles of the Golgi and endosomal-lysosomal network. 


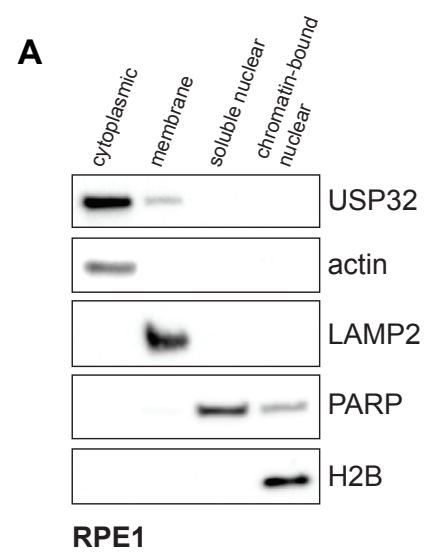

B
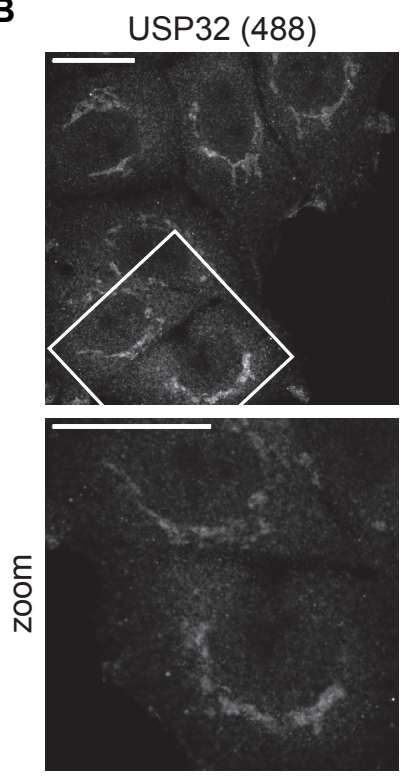

USP32 (488)
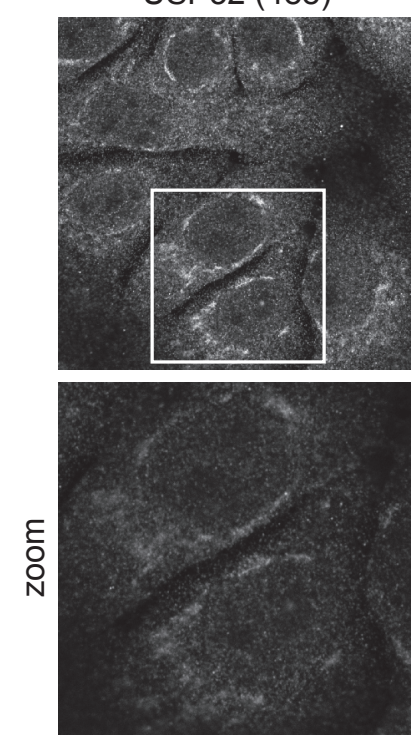

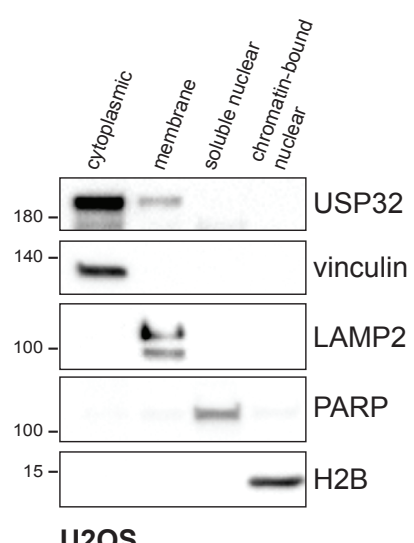

U2OS

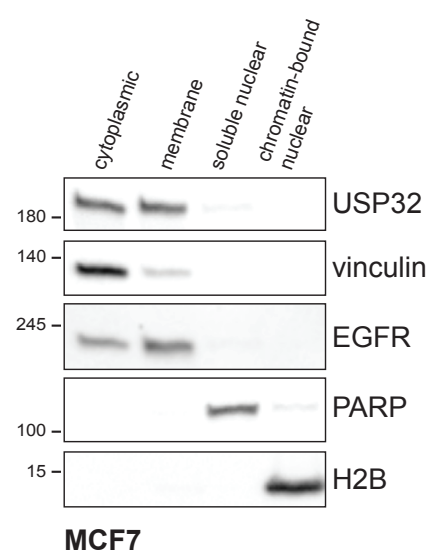

merge + DAPI
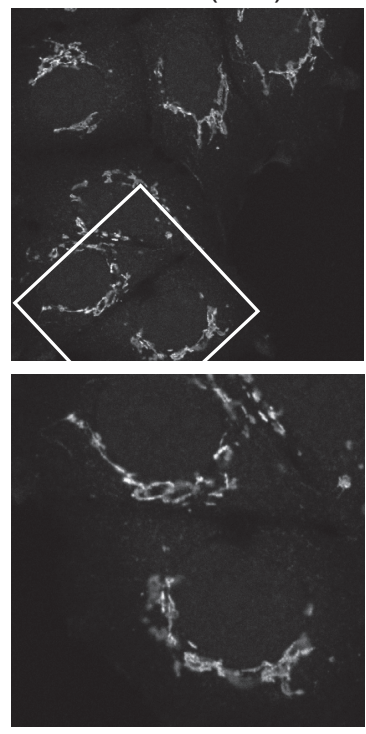

LAMP1 (647)
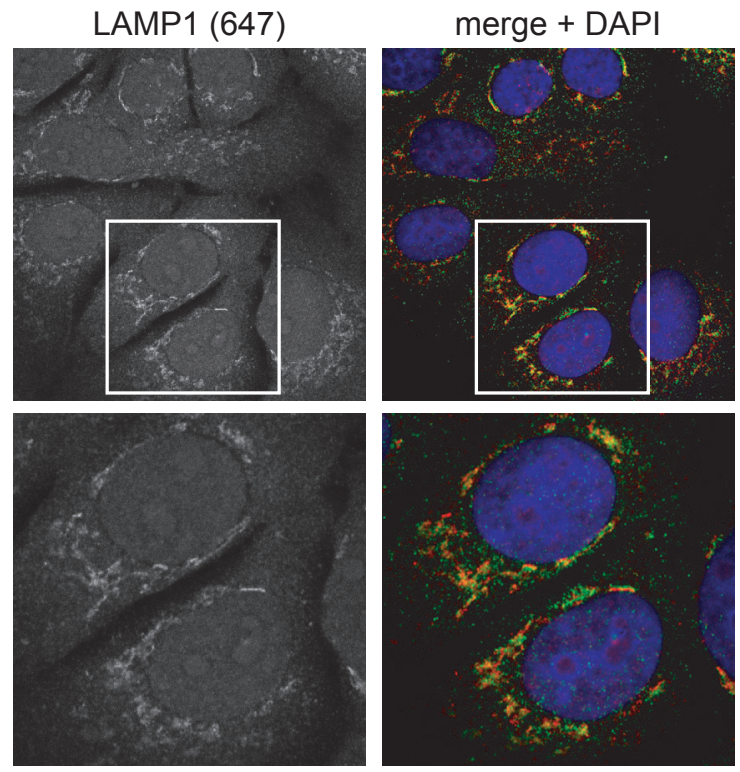

Figure 3-3: USP32 is localized at membranes in the perinuclear region of cells. A USP32 is present in the cytoplasmic and membrane fraction in different cell types. Subcellular fractionation of RPE1, U2OS and MCF7 cells was analyzed by Western blotting. B Endogenous USP32 localizes at the Golgi apparatus and lysosomes. MCF7 cells were co-stained for USP32 and either the Golgi marker GM130 or the lysosomal marker LAMP1. Scale bar $=20 \mu \mathrm{m}$ 


\subsection{Identification of USP32 substrates (diGly IP)}

The physiological function of USP32 is only emerging. To identify potential USP32 substrates and to further the understanding of USP32-regulated cellular processes, a Stable IsotopeLabeling of Amino acids in Cell culture (SILAC)-based quantitative ubiquitinome analyses (diGly remnant profiling) was performed.

The non-radioactively metabolic labeling of lysines and arginines was chosen for relative quantitative comparison. Furthermore, in this approach the lysates of the different conditions were combined after the lysis to minimize differences in sample preparation. The amino acids were incorporated in all proteins and gave a unique spectral signature. The proteins were identified based on the spectra. For relative quantification, the ratios of the according SILAC pairs were used.

The schematic experimental workflow is shown in Figure 3-4 A and described in detail in 6.2.10.1. In brief, RPE1 NHT and USP32KO cells were grown for two weeks in DMEM suitable for SILAC labeling containing either light (KORO) or heavy (K8R10) lysine and arginine (incorporation test $>95 \%$ ). The ubiquitinome of both cell lines was analyzed under basal conditions. The immunoaffinity purification (IAP) for ubiquitinated peptides was performed using antibodies that recognize the remnant diGly motif after a tryptic digest. The samples were subjected to liquid chromatography and mass spectrometry (LC-MS/MS) analysis in collaboration with Florian Bonn (Institute of Biochemistry II, Goethe University Frankfurt). Normalization of the relative abundance of enriched diGly peptides was done against the whole cell proteome of input samples (= whole cell lysate directly after lysis).

The volcano plot in Figure 1-1 B depicts the identified diGly remnant peptides with corresponding fold change (expressed in log2 ratios) and p-values (expressed in -log). Comparison was done between USP32KO (heavy) and NHT (light) samples. Taking into account that a DUB cleaves ubiquitin modifications, which is not happening in knockout cells anymore, only peptides with positive log2 ratio could be direct substrates of USP32. Identified proteins with $\log 2$ ratio $\geq 0.6$ and - $\log p$-value $\geq 2$ were considered as significantly enriched. An accumulation of several proteins associated with the endosomal and lysosomal system was observed. Figure 3-4 C describes the four most interesting hits Rab7, LAMTOR1, Rab11 and TMEM192 with information on their identified modification and cellular function. The schematic cartoon (Figure 3-4 C) highlights their cellular localization and relative positions of the ubiquitination. 
A

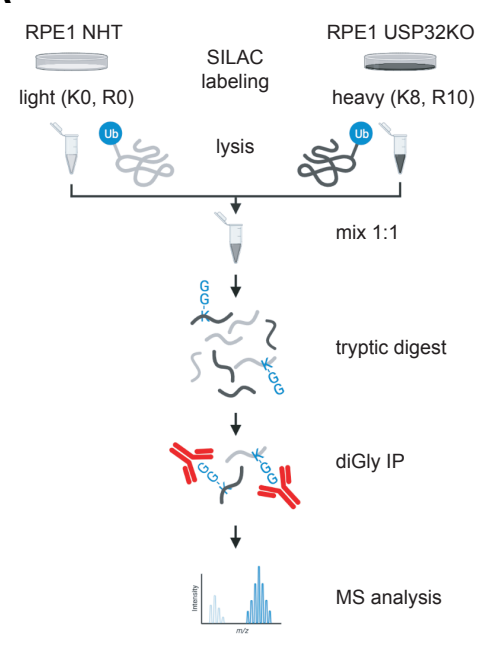

B

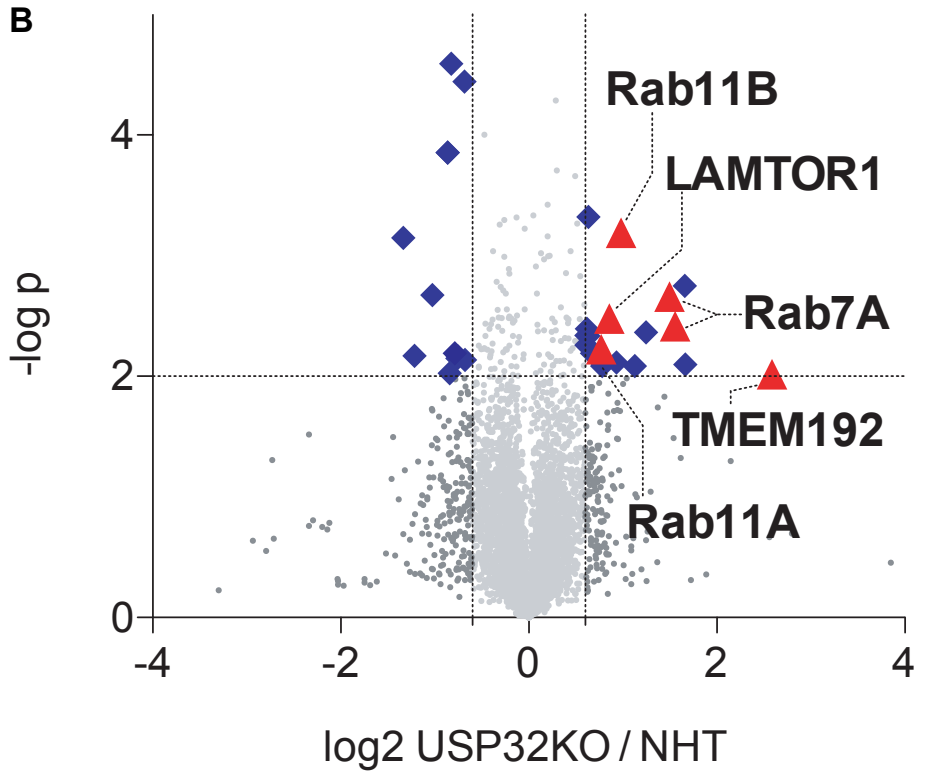

C

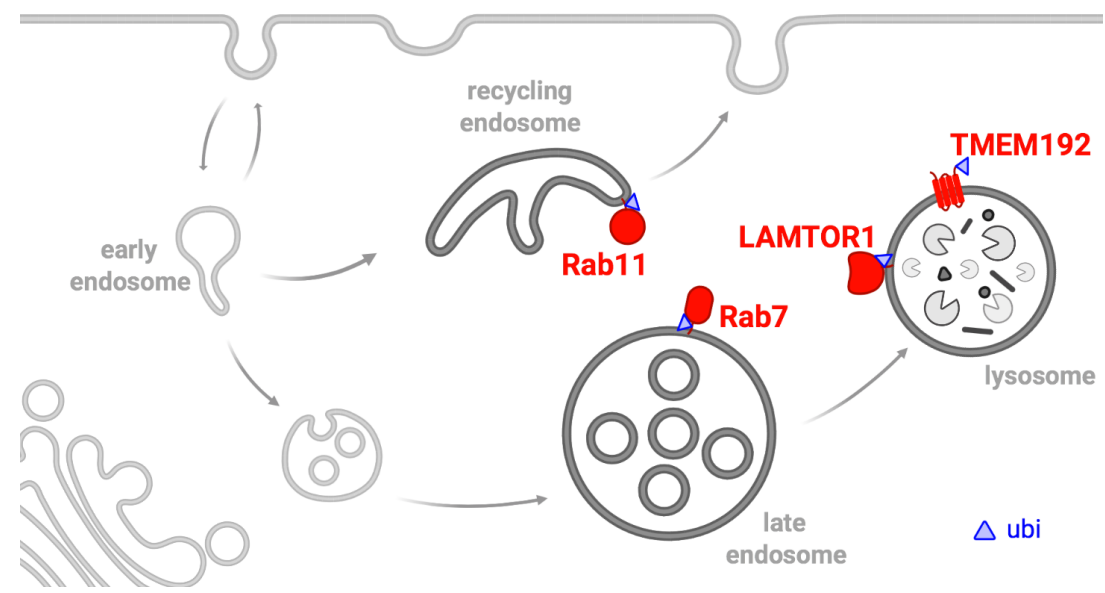

\begin{tabular}{c|ccccc} 
& $\log 2(\mathrm{KO} / \mathrm{NHT}) /$ & & & position & cellular function \\
\hline Rab7 & $1.50 / 2.66$ & $\mathrm{~K} 191$ & unstructured C-terminus, close to lipidation site(s) & key regulator of late endosomal trafficking \\
Rab7 & $1.56 / 2.41$ & $\mathrm{~K} 194$ & unstructured C-terminus, close to lipidation site(s) & key regulator of late endosomal trafficking \\
LAMTOR1 & $0.86 / 2.48$ & $\mathrm{~K} 20$ & unstructured N-terminus, close to lipidation site(s) & component of lysosomal Ragulator complex \\
Rab11A & $0.77 / 2.23$ & K179 & unstructured C-terminus, close to lipidation site(s) & key regulator of recycling endosomes \\
Rab11B & $0.98 / 3.19$ & K179 & unstructured C-terminus, close to lipidation site(s) & key regulator of recycling endosomes \\
TMEM192 & $2.59 / 2.02$ & K246 & unstructured cytosolic C-terminus & uncharacterized lysosomal transmembrane protein
\end{tabular}

Figure 3-4: Ubiquitinome analyses determined potential USP32 substrates involved in endosomal trafficking as well as lysosomal proteins. A Experimental setup of SILAC-based quantitative ubiquitinome analyses in RPE1 cells to determine potential USP32 substrates. B Volcano plot depicting the identified diGly remnant peptides with corresponding fold change (expressed in log2 ratios) and p-values (expressed in -log). Comparison was done between USP32KO (heavy) and NHT (light). Identified proteins with log2 ratio $\geq 0.6$ and -log p-value $\geq 2$ were considered as significantly enriched and labeled in blue. Significantly enriched proteins in the endosomal-lysosomal system interesting for further validation were labeled in red. Data was obtained from one SILAC sample set with three technical replications. C Cartoon and table showing the interesting endosomal-lysosomal proteins, the identified regulated diGly site(s) in USP32KO cells and their relative position within the protein sequence and their main compartment localization.

Ubiquitination has multiple roles including the tagging of proteins with K48 linked chains for proteasomal degradation. It is known that DUBs belonging to the USP family have low ubiquitin chain linkage specificity but rather substrate specificity. To investigate whether USP32 cleaves a proteolytic chain from the interesting hits, levels of all four proteins were detected by 
immunoblotting without and with treatment with MG132 for proteasomal inhibition (Figure 3-5). Protein abundance did not change in USP32KO cells compared to NHT cells. Furthermore, the proteome analysis of the diGly IP (3.4) did not reveal any significant changes in overall protein levels upon USP32 depletion (data not shown). Both Western blot and whole cell proteome analysis of the diGly IP suggested that USP32 does not cleave a proteolytic signal and does not directly regulate protein levels of Rab7, LAMTOR1, TMEM192 and Rab11.

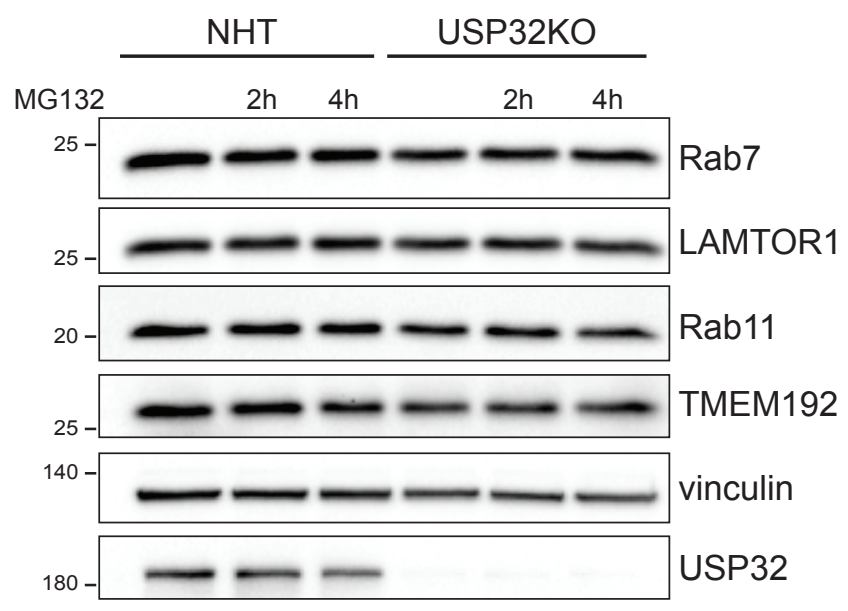

Figure 3-5: USP32 cleaves a non-proteolytic signal from endosomal-lysosomal proteins. RPE1 NHT and USP32KO cells were either untreated or treated for $2 \mathrm{~h}$ and $4 \mathrm{~h}$ with $10 \mu \mathrm{M} \mathrm{MG132}$ and protein levels were detected by Western blotting.

Summarizing the first findings, (I) the deubiquitinase USP32 was localized in the endosomallysosomal system and could be associated with membranes. (II) Potential substrates of the endosomal and lysosomal system were identified. (III) USP32 did not cleave a proteolytic signal on these proteins. Two of the four interesting hits, Rab7 and LAMTOR1, were picked for further studies in this work.

\subsection{Characterization of Rab7 as USP32 substrate}

Rab7 is the key regulator of endosomal and lysosomal trafficking. It has several important functions e.g. vesicle transport along microtubules or vesicle fusion (see introduction). Rab7 was shown to be ubiquitinated on lysine 38 by the E3 ligase Parkin, thereby regulating its protein levels and activity (Song 2016). Though ubiquitination at K191 and K194 was reported before, the function of this modification was not elucidated so far. Neither was a DUB described to deubiquitinate Rab7 when the following investigations were started. This presented Rab7 as the most interesting hit for further validation and characterization.

\subsubsection{Interaction of USP32 and Rab7}

In order to show enzymatic activity towards its substrate, USP32 was supposed to interact with Rab7. The interaction was confirmed by a GFP-trap IP and subsequent immunoblotting (Figure 3-6 A). GFP-Rab7 was co-expressed with HA-USP32 in HEK293 cells and immunoprecipitated. Expression of GFP alone in presence of USP32 was used as negative IP control. USP32 was detected in the IP sample using an antibody against its HA tag. 


\subsubsection{USP32-sensitive Rab7 ubiquitination}

To confirm the results from the mass spectrometry approach that Rab7 is modified in a USP32dependent manner, a His-ubiquitin (His-ubi) pulldown (PD) was performed. His-tagged ubiquitin was co-expressed with Myc-Rab7 in HEK293 cells either in presence or in absence of overexpressed HA-USP32. His-ubi was enriched with Ni-NTA beads and samples were subjected to immunoblotting. The modification of Rab7 was detected with a Myc-specific antibody. In cells also transfected with HA-USP32 the Rab7 modification, visible as higher molecular smear on a Western blot, was significantly decreased (Figure 3-6 B).

A

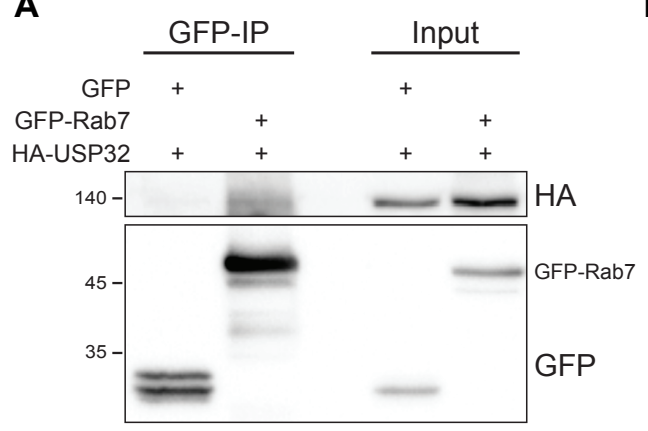

B

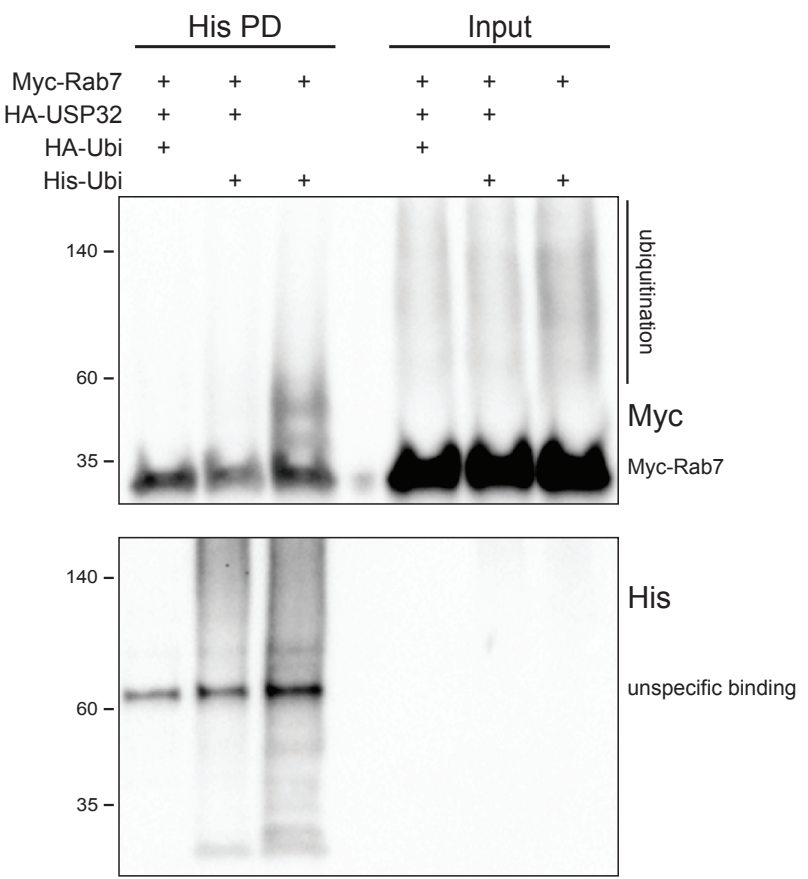

Figure 3-6: Rab7 shows interaction and USP32-sensitive ubiquitination A HA-USP32 coimmunoprecipitated with GFP-Rab7 in a GFP-trap IP and was detected by immunoblotting. B Rab7 ubiquitination was decreased with overexpressed USP32. His-ubiquitin tagged proteins were pulled down from HEK cells co-expressing Myc-Rab7 with or without HA-USP32 and probed against Myc. Myc-Rab7 bands in input samples were overexposed.

\subsubsection{Rab7 localization at late endosomes and lysosomes}

The combination of several post-translational modifications like phosphorylation, lipidation and ubiquitination modulates Rab7 function and its ability to orchestrate trafficking at the late endosomes. After identification and confirmation of the non-proteolytic USP32-sensitive ubiquitination of Rab7 K191/194 the function of this modification was investigated.

Ubiquitination was reported to mediate Rab7 membrane association (Song et al., 2016). Therefore the first experiment addressed the localization of Rab7 at the late endosomal and lysosomal compartment. In collaboration with Florian Steinberg (Center for Biological Systems Analysis, University of Freiburg) immunofluorescence staining with specific antibodies against Rab7 and the lysosomal marker protein LAMP1 was applied to RPE1 NHT and USP32KO cells. Representative images from confocal microscopy are shown in Figure 3-7 A. There was no obvious change of Rab7 localization relative to LAMP1 positive structures upon depletion of USP32 in comparison to the control. 


\subsubsection{Rab7 activity and RILP binding}

Parkin loss of function displayed a decrease of Rab7 K38 modification and effector binding capability. To test the hypothesis that depletion of USP32 and concomitant Rab7 ubiquitination at K191/194 results in altered Rab7 activity and effector binding, GTP-bound active Rab7 was enriched with the help of its effector RILP in collaboration with Florian Steinberg (Center for Biological Systems Analysis, University of Freiburg). Lysates from RPE1 NHT and USP32KO cells were incubated with recombinant GST-RILP. A GST pulldown was performed and bound Rab7 was detected on a Western blot (Figure 3-7 B). Increased binding of Rab7 to RILP in the USP32KO sample hints to a higher activity of Rab7 or higher affinity to RILP in situation without USP32.

A

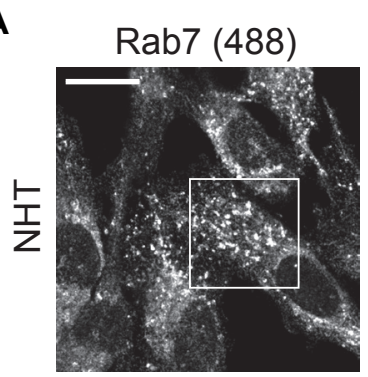

LAMP1 (594)

merge+DAPI

zoom
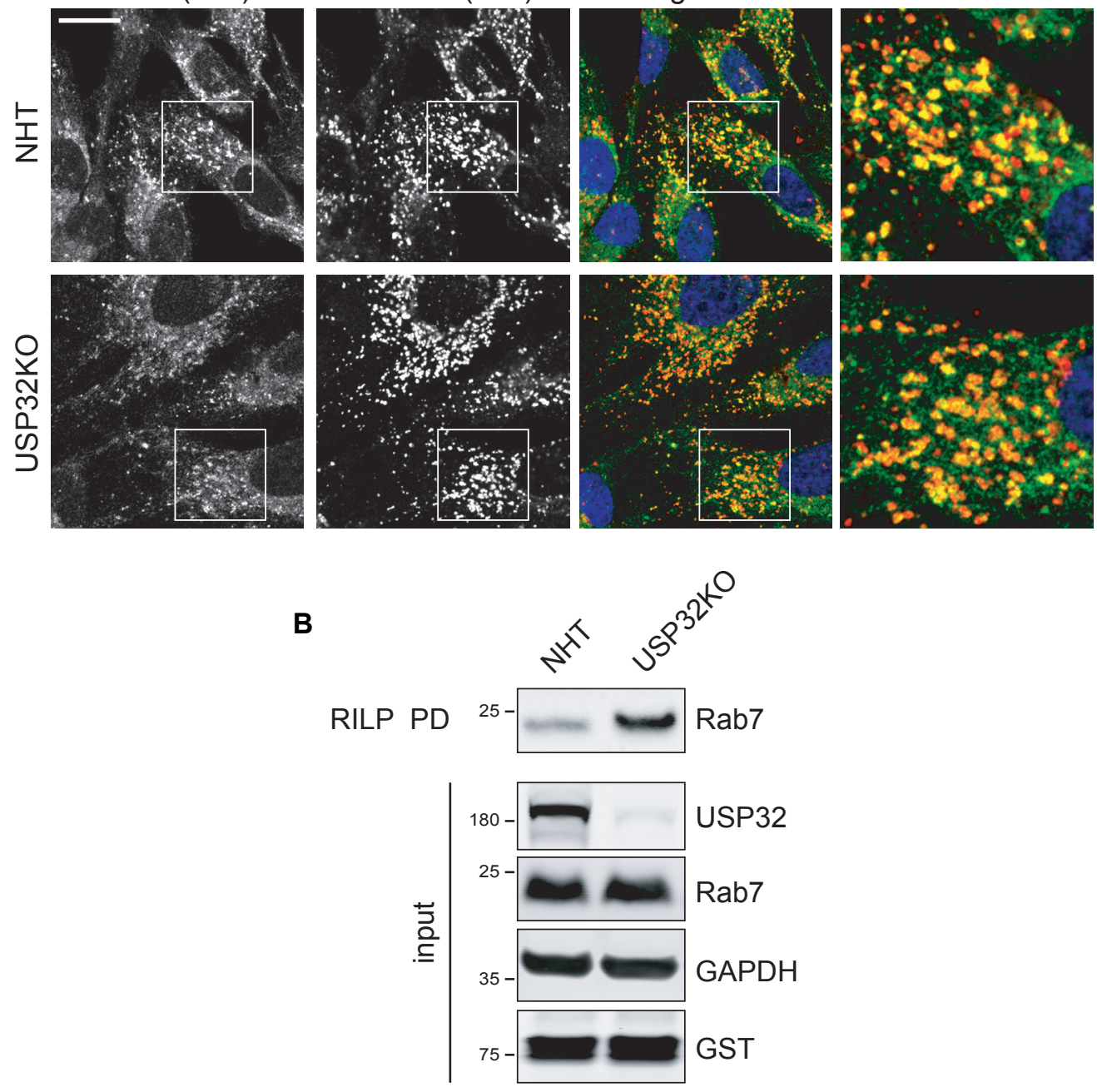

Figure 3-7: USP32-sensitive Rab7 ubiquitination alters Rab7 activity but not its localization. A Endogenous Rab7 did not change its localization relative to LAMP1 stained lysosomes in USP32-deficient RPE1 cells. RPE1 NHT and USP32KO cells were stained for Rab7 and LAMP1. Scale bar $=20 \mu \mathrm{m}$ B RILP preferentially bound to ubiquitinated Rab7. Lysates from RPE1 NHT and USP32KO cells were probed with immobilized GST-RILP protein. GSTRILP bound Rab7 was detected by Western blotting. 


\subsubsection{Rab7 mediated Cl-M6PR trafficking}

A well described trafficking pathway in the endosomal and lysosomal system is the transport of the cation-independent mannose 6-phosphate receptor (CI-M6PR).

Cl-M6PR cycles between trans-Golgi network (TGN), endocytic compartment and plasma membrane. The retromer complex mediates the return of unoccupied CI-M6PR to the TGN and allows sorting of newly synthesized hydrolases to acidic late endosomes where the cargo dissociates (Seaman, 2004). (Rojas et al., 2008) showed that tethering the complex to endosomal membranes requires association with active GTP-bound Rab7. In contrast, dissociation from membranes causes inhibition of Cl-M6PR retrograde transport, and missorting of the acid hydrolase cathepsin D.

To test whether higher activity of Rab7 caused by USP32 depletion affects CI-M6PR trafficking, the retromer-mediated internalization of the receptor over time was investigated with an antibody-feeding assay. Again in collaboration with Florian Steinberg (Center for Biological Systems Analysis, University of Freiburg), RPE1 NHT and USP32KO cells in culture were incubated with a specific antibody against the luminal part of the CI-M6PR. The transport to the perinuclear region was observed by IF co-staining of the internalized antibody and the transGolgi marker TGN46. Figure 3-8 A shows representative microscopy images and the quantification of the Pearson's correlation coefficient between CI-M6PR and TGN46. In cells lacking USP32 the co-localization was increased significantly after $15 \mathrm{~min}$.

Similar effects were observed under steady state conditions when CI-M6PR localization was investigated relative to the Golgi marker Giantin in untreated RPE1 cells by immunofluorescence microscopy. Their co-localization was quantified with the Pearson's correlation coefficient and revealed that USP32KO results in higher overlap of both CI-M6PR and Giantin signals (Figure 3-8 B). 

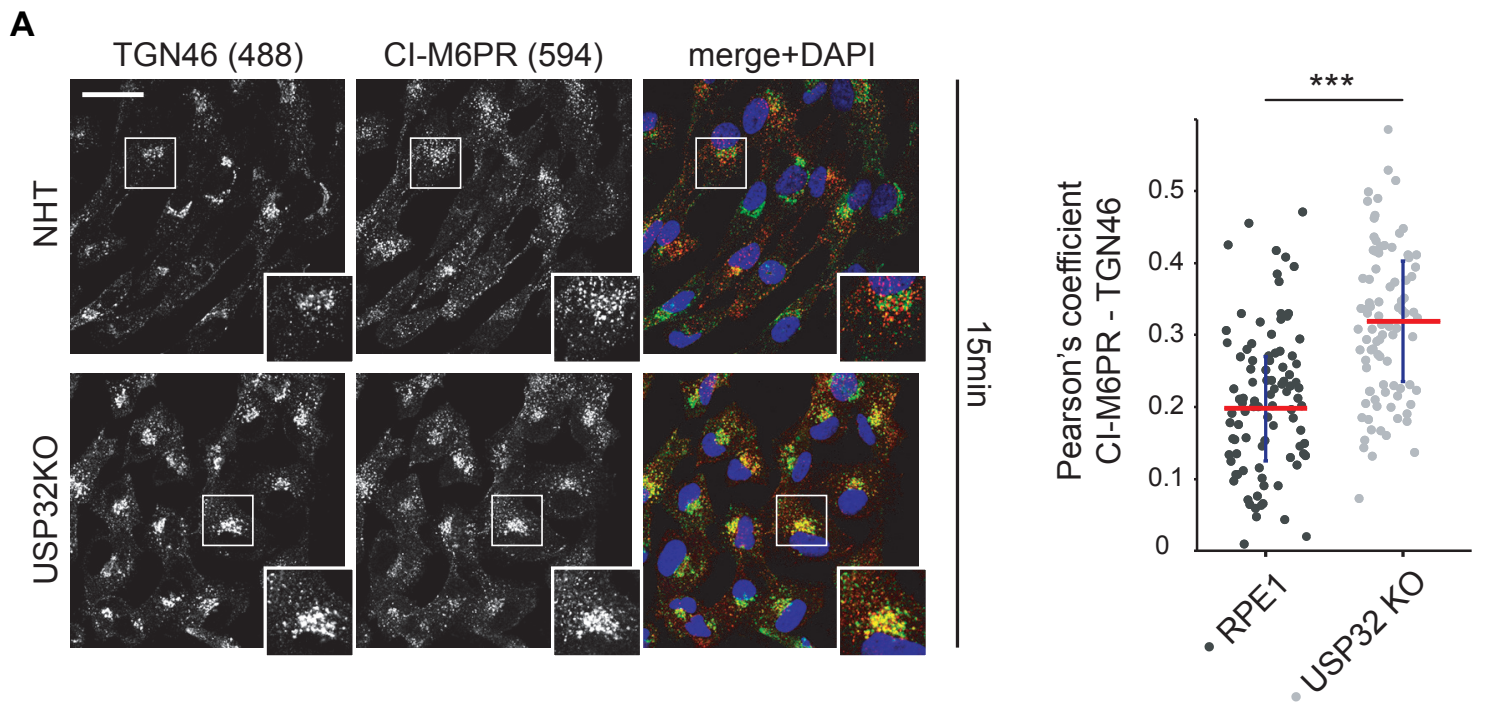

B
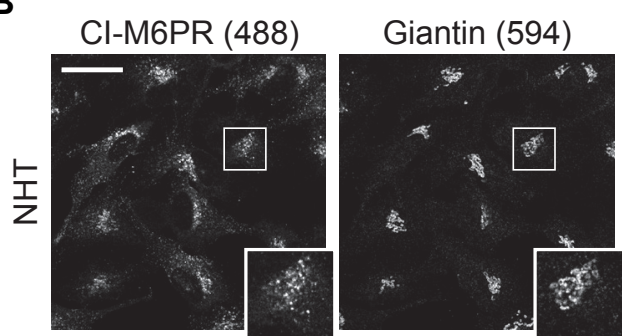

merge+DAPI
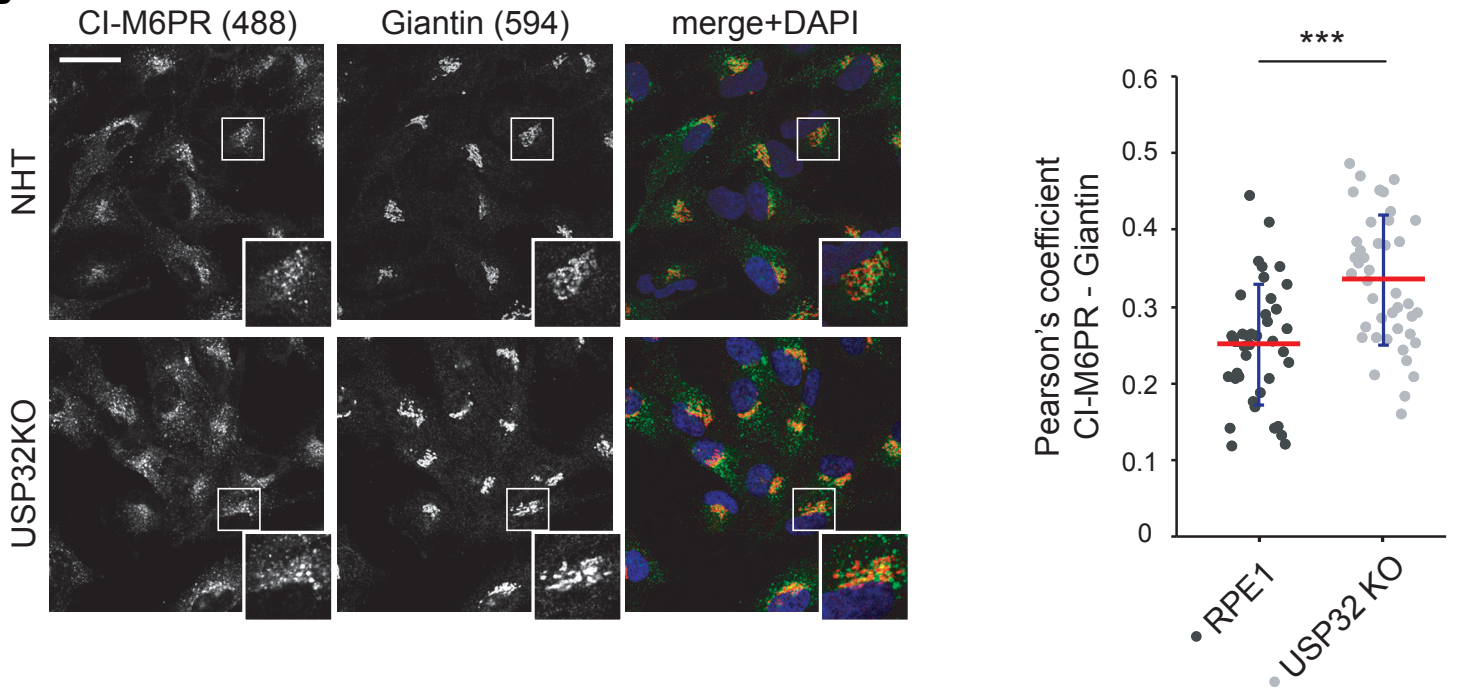

Figure 3-8: Rab7 mediated retrograde trafficking of CI-M6PR is enhanced upon USP32 depletion. A RPE1 NHT and USP32KO cells were incubated with an antibody against the extracellular (luminal) domain of endogenous $\mathrm{Cl}-\mathrm{M} 6 \mathrm{PR}$ for $15 \mathrm{~min}$ at $37^{\circ} \mathrm{C}$, followed by fixation and staining for the internalized antibody and TGN46. Colocalization was quantified by the Pearson's correlation coefficient (per cell) between CI-M6PR and TGN46 across two independent experiments with indicated mean $\pm S D, . ._{\text {Scale bar }}=15 \mu \mathrm{m}$ B Cl-M6PR localization at the TGN was increased in USP32KO cells. RPE1 NHT and USP32KO cells were stained for CI-M6PR and Giantin. Co-localization was quantified by the Pearson's correlation coefficient (per cell) between Cl-M6PR and Giantin with indicated mean \pm SD,. Scale bar $=15 \mu \mathrm{m}, \quad * * * \mathrm{p}<0.0001$ in a two-tailed, unpaired Student's $\mathrm{t}$ test between the indicated conditions

\subsubsection{Hydrolase sorting}

The previous data described an enhancement of Rab7 activity and subsequently retromermediated Cl-M6PR transport upon loss of USP32. This transport process is necessary to maintain the levels of lysosomal hydrolase in the late endosomal and lysosomal compartment. Consequentially, the next experiments examined whether USP32KO in cells alters hydrolase sorting resulting in changes of the lysosomal content. 


\subsubsection{Lysosome proteome}

For this, a SILAC based mass spectrometry approach was chosen to analyze the proteome of purified lysosomes. The schematic experimental workflow is shown in Figure 3-9 A and described in detail in 6.2.8. In brief, U2OS ctrl and USP32KO cells were grown for two weeks in DMEM suitable for SILAC labeling containing either light (KORO) or heavy (K8R10) lysine and arginine (incorporation test $>95 \%$ ). The lysosomes were enriched by density gradient centrifugation and the respective fraction was subjected to in-gel digest. The subsequent LCMS/MS analysis was done in collaboration with Florian Bonn (Institute of Biochemistry II, Goethe University Frankfurt).

The volcano plot in Figure 3-9 B depicts the identified peptides with corresponding fold change (expressed in log2 ratios) and p-values (expressed in -log). Comparison was done between USP32KO (heavy) and ctrl (light) samples. Identified proteins with log2 ratio $\leq-0.6$ or $\geq 0.6$ and $p$-value $\geq 0.05$ were considered as significantly enriched. An accumulation of several acid hydrolases (cathepsins (Cath)) was observed in lysosomes from USP32KO cells. In addition, the lysosomal protein RagC/D and TMEM192 were significantly enriched in USP32KO lysosomes. USP32 itself was detected in the lysosomal fraction with low abundance serving as a confirmation of USP32 lysosomal localization (see Figure 3-3 B). Figure 3-9 C shows all interesting identified proteins and their respective values. The whole cell proteome showed no interesting significant changes in protein levels (data not shown).

\subsubsection{Lysosome content on immunoblot}

The finding of the lysosome proteome could be confirmed on a complementary Western blot. Like for the MS approach, the lysosomal fraction was enriched but from unlabeled RPE1 NHT and USP32KO cells by density gradient centrifugation. The samples with purified lysosomes and whole cell lysate were prepared for SDS-PAGE and analyzed by immunoblotting (Figure 3-9 D). An increase in cathepsin $Z$ levels in lysosomes upon USP32 depletion was detected. Notably, also the overall abundance of cathepsin Z in the lysate was higher in RPE1 USP32KO cells in this experiment. This result was contradictory to the full proteome analysis of both diGly IP (from RPE1) and lysosomal proteome (from U2OS) experiments. 
A

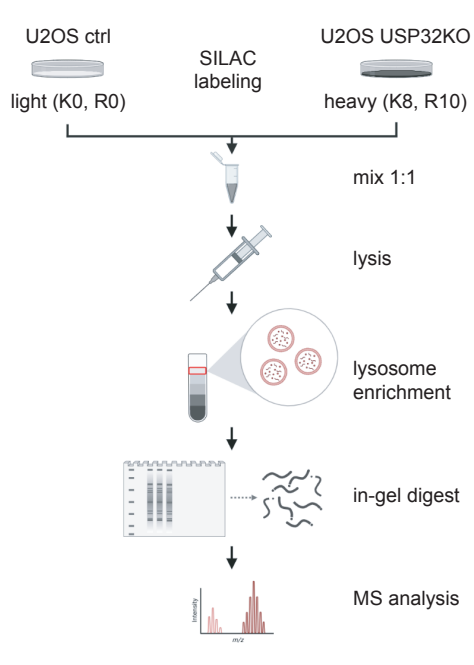

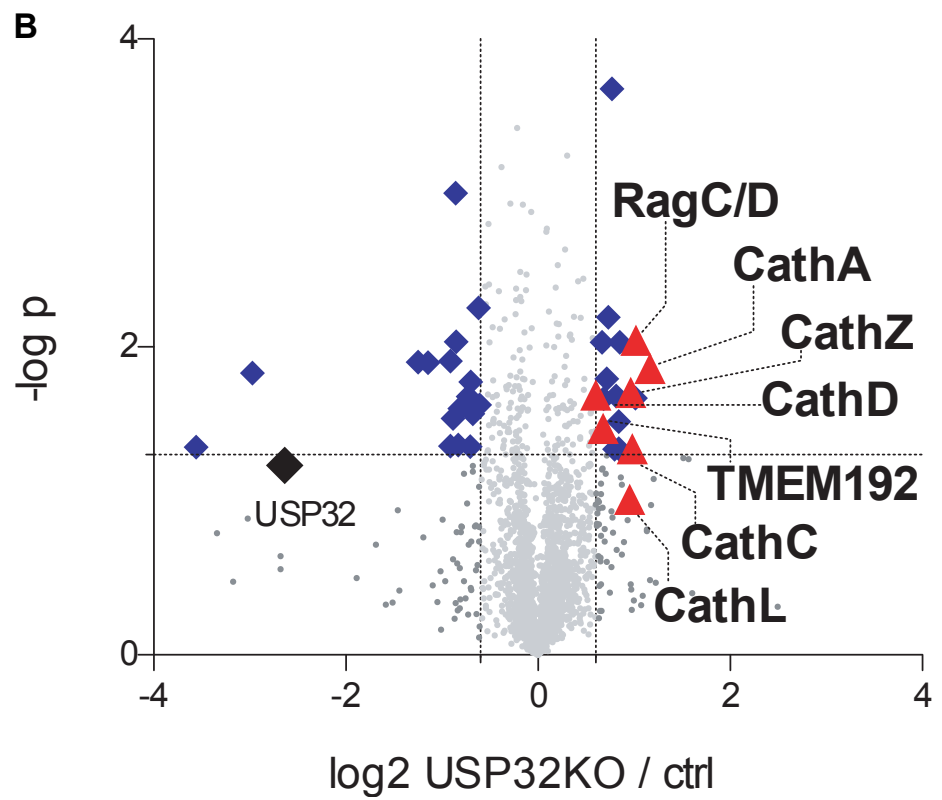

C

lysosome proteome

\begin{tabular}{l|ccc|ccc}
\multicolumn{1}{c|}{ Protein } & $\log 2(\mathrm{KO} / \mathrm{NHT})$ & $-\log (\mathrm{p})$ & & \multicolumn{1}{c}{ Protein } & $\log 2(\mathrm{KO} / \mathrm{NHT})$ & $-\log (\mathrm{p})$ \\
\hline CTSA & $\mathbf{1 , 1 6}$ & $\mathbf{1 , 8 6}$ & & S100A10 & $-0,61$ & 1,62 \\
RAGC/D & $\mathbf{1 , 0 2}$ & $\mathbf{2 , 0 4}$ & & GALK1 & $-0,62$ & 2,25 \\
TPP1 & 1,02 & 1,67 & & SDK2 & $-0,68$ & 1,57 \\
CTSC & 0,98 & 1,34 & & ACTR1B & $-0,70$ & 1,77 \\
CTSZ & 0,96 & 1,70 & & ECE1 & $-0,71$ & 1,35 \\
PI4K2A & 0,85 & 2,03 & & RHOF & $-0,73$ & 1,68 \\
FAM3C & 0,84 & 1,34 & & IGSF3 & $-0,81$ & 1,60 \\
GGH & 0,84 & 1,51 & & AARSD1 & $-0,83$ & 1,36 \\
PLBD2 & 0,81 & 1,68 & & ITGA6 & $-0,85$ & 2,04 \\
GBA & 0,80 & 1,34 & & ITGA5 & $-0,86$ & 3,00 \\
STX10 & 0,77 & 3,68 & & ITGB3 & $-0,88$ & 1,54 \\
CRYAB & 0,73 & 2,19 & & STAM & $-0,91$ & 1,91 \\
ABCD4 & 0,71 & 1,79 & & TBC1D10A & $-0,91$ & 1,36 \\
ARHGAP31 & 0,68 & 1,66 & & CLDN3/4/6/9 & $-1,14$ & 1,90 \\
TMEM192 & 0,68 & 1,47 & & ALYREF & $-1,25$ & 1,90 \\
TMEM106B & 0,67 & 2,03 & & APOB & $-2,97$ & 1,83 \\
CTSD & 0,60 & 1,69 & & TM9SF1 & $-3,56$ & 1,35
\end{tabular}




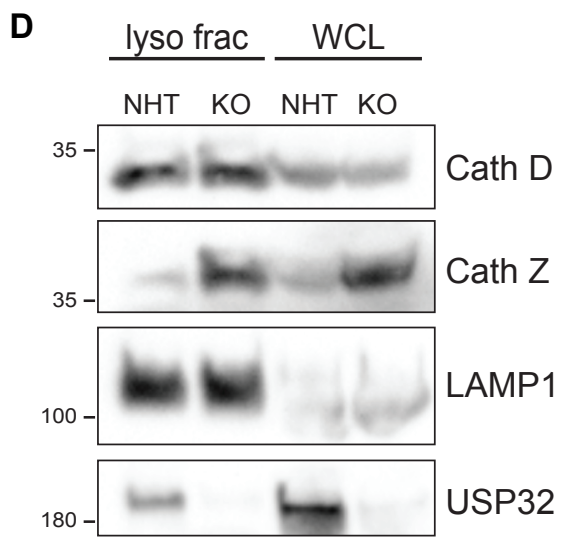

RPE1

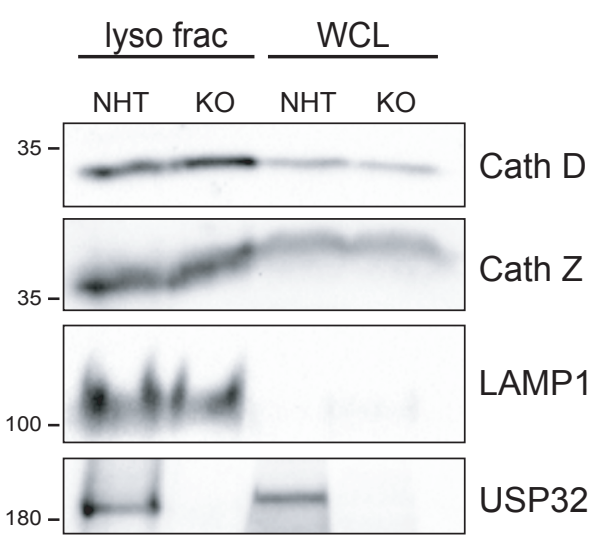

U2OS

Figure 3-9: Sorting of lysosomal enzymes is impaired in USP32-deficient cells. A Experimental setup of SILAC-based quantitative lysosomal proteome analyses in U2OS cells. B Volcano plot depicting the identified peptides with corresponding fold change (expressed in log2 ratios) and p-values (expressed in -log). Comparison was done between USP32KO (heavy) and ctrl (light). Identified proteins with $\log 2$ ratio $\geq 0.6$ and $-\log p$-value $\geq 1.3$ were considered as significantly enriched and labeled in blue. Lysosomal proteins that are interesting in the USP32 context were labeled in red. Data was obtained from one SILAC sample set with three technical replications. C Table listing all significantly up- or downregulated proteins in the lysosomal proteome with their respective $\log 2$ ratios and $-\log (\mathrm{p})$ values. Interesting proteins were marked in bold. Cathepsin = CTS D Lysosomes from RPE1 and U2OS NHT/ctrl or USP32KO cells were enriched by density gradient centrifugation. Proteins levels of the lysosomal fraction as well as the whole cell lysates (WCL) were detected by Western blotting.

\subsubsection{Hydrolase secretion}

Altered Rab7 and retromer function was described to impair the trafficking of lysosomal enzymes resulting in their secretion into the cell culture medium (MacDonald et al., 2014; Modica et al., 2017; Rojas et al., 2008). These publications associated not only Rab7 function itself, but also the post-translational modification palmitoylation and, furthermore, the DUB USP8 to interference with retromer mediated trafficking and sorting.

The presented data from lysosome proteome and complementary Western blot analysis suggested a more efficient sorting of lysosomal hydrolases due to higher Rab7 activity upon USP32 depletion. Would USP32 then also have an effect on hydrolase secretion? To address this question, again a SILAC based mass spectrometry experiment together with complementary immunoblotting was performed.

\subsubsection{Secretome}

A SILAC based MS approach was chosen for the investigation of secreted proteins. The schematic experimental workflow is shown in Figure 3-10 A and described in detail in 6.2.10.2. In brief, RPE1 NHT and USP32KO cells were grown for two weeks in DMEM suitable for SILAC labeling containing either light (KORO) or heavy (K8R10) lysine and arginine (incorporation test $>95 \%$ ). Secreted proteins were enriched with StrataClean beads from overnight conditioned SILAC medium without FBS. Whole cell lysate was prepared for later full proteome analysis. The samples were subjected to in-gel digest. The subsequent LC-MS/MS analysis was done in collaboration with Florian Bonn (Institute of Biochemistry II, Goethe University Frankfurt).

Comparison was done between USP32KO (heavy) over NHT (light) samples. Identified proteins with $\log 2$ ratio $\leq-0.6$ or $\geq 0.6$ and $p$-value $\geq 0.01$ were considered as significantly down- or 
upregulated upon USP32 depletion. Neither in the enriched nor in the decreased fraction were lysosomal hydrolases or any other proteins of interest. Proteins with $\log 2 \geq 0.6$ were mostly mitochondrial proteins and considered as contamination. Proteins with $\log 2 \leq-0.6$ were mostly extra cellular matrix proteins coming from the used FBS (Figure 3-10 B). Since the FBS from SILAC medium is not isotope-labeled the proteins were identified as light peaks and appeared to be decreased in the heavy USP32KO sample.

Interestingly, the full proteome analysis of this experiment revealed, in contrast to the two previous proteome analyses of diGly IP and lysosomal proteome, an enrichment of cathepsin Z (Figure 3-10 B). This finding would go along with the higher cathepsin $Z$ levels in the immunoblot experiment in Figure 3-9 D.

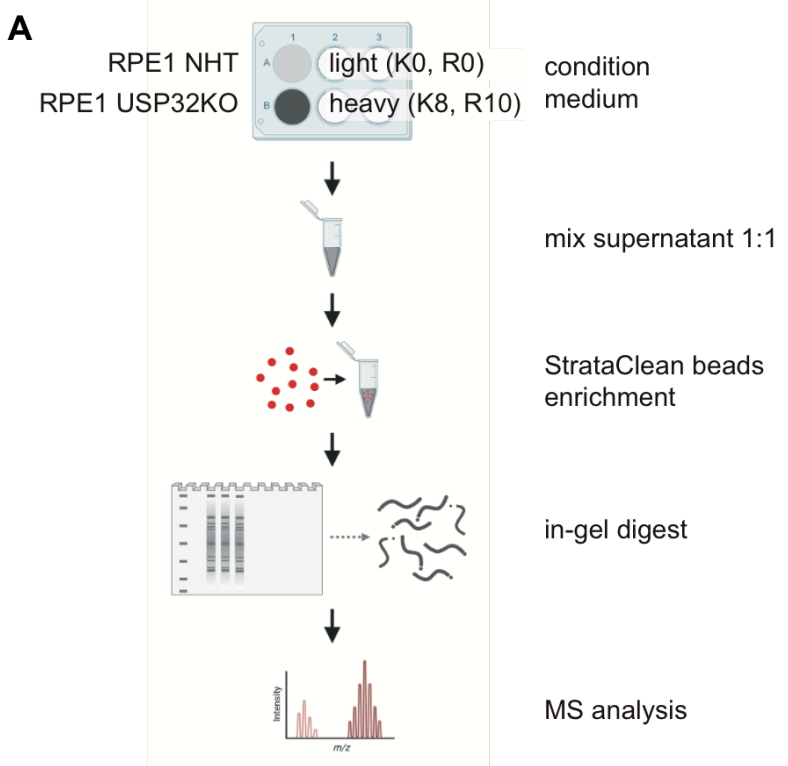

B

\begin{tabular}{c|cc}
\multicolumn{3}{c}{ full proteome of secretome } \\
Protein & $\log 2(\mathrm{KO} / \mathrm{NHT})$ & $-\log (\mathrm{p})$ \\
\hline CTSZ & 1,53 & 1,56 \\
THY1 & 0,72 & 2,53 \\
RNF170 & 0,71 & 1,30 \\
SMARCAD1 & 0,70 & 1,51 \\
KIF3A & 0,68 & 2,34 \\
PGM5 & 0,62 & 1,90 \\
SERPINB2 & 0,61 & 2,87 \\
B4GAT1 & $-0,60$ & 2,20 \\
SLC25A30 & $-0,68$ & 2,22 \\
LYZ & $-0,70$ & 1,38 \\
DCD & $-2,41$ & 1,37 \\
DSC1 & $-2,80$ & 1,85 \\
SHROOM3 & $-3,69$ & 1,55
\end{tabular}

\begin{tabular}{c|cc} 
Protein & $\begin{array}{c}\text { secretome } \\
\text { log2 }(\mathrm{KO} / \mathrm{NHT})\end{array}$ & -log $(\mathrm{p})$ \\
\hline PHB & 1,33 & 2,59 \\
ATP5B & 1,30 & 2,03 \\
ATP5A1 & 1,30 & 2,19 \\
SLC25A3 & 1,24 & 2,16 \\
ATP5O & 1,23 & 2,71 \\
PHB2 & 1,14 & 2,49 \\
ATP5F1 & 1,09 & 2,00 \\
ATP5C1 & 1,02 & 2,01 \\
HSPD1 & 0,97 & 2,80 \\
ACOT1/2 & 0,94 & 2,10 \\
ACAT1 & 0,94 & 3,62 \\
PTPN23 & 0,85 & 2,86 \\
MARS & 0,85 & 2,25 \\
ACO2 & 0,74 & 2,40 \\
TRPV1 & 0,72 & 2,12 \\
HSP90B1 & 0,66 & 2,06 \\
EEA1 & 0,66 & 2,80 \\
EFTUD2 & 0,65 & 2,49 \\
\hline ANGPT1 & $-0,69$ & 2,60
\end{tabular}




\begin{tabular}{c|cc} 
IGSF8 & $-0,70$ & 2,13 \\
NLGN1/2 & $-0,72$ & 2,16 \\
CDH6 & $-0,90$ & 2,34 \\
ISOC1 & $-0,91$ & 2,12 \\
FSTL3 & $-0,93$ & 2,26 \\
BGN & $-1,14$ & 2,26 \\
IL6 & $-1,20$ & 2,45 \\
CGREF1 & $-1,29$ & 2,61 \\
DCD & $-1,69$ & 2,00 \\
APOB & $-2,71$ & 2,39 \\
COL1A1 & $-2,82$ & 2,04 \\
DDX46 & $-3,11$ & 2,25 \\
APOM & $-4,87$ & 2,17 \\
COL2A1 & $-4,95$ & 2,28
\end{tabular}

Figure 3-10: Enhanced secretion of lysosomal hydrolases is not detected. A Experimental setup of SILACbased quantitative secretome analyses in RPE1 cells. B Table listing all significantly up- or downregulated proteins in the full proteome and secretome with their respective $\log 2$ ratios and $-\log (p)$-values. Cathepsin $=$ CTS

\subsubsection{Secreted proteins on immunoblot}

Several experiments were performed to confirm the mass spectrometry data with a complementary Western blot with two different experimental setups to enrich proteins in the conditioned supernatant: (I) concentration with Amicon Ultra centrifugal filters (3 kDa cutoff) or (II) enrichment with StrataClean beads. With both methods enrichment was achieved which was verified by protein concentration measurement. But the Western blot analyses showed either no signal for cathepsin $D$ and cathepsin $Z$ in the supernatant sample or results were inconsistent. No conclusions could be drawn from these experiments.

\subsubsection{Lysosomal degradation efficiency (DQ-BSA assay)}

Acidic hydrolases like cathepsins are the catalytic active components enclosed in lysosomes, one of the main degradative machinery of the cell. Changes in the cathepsin levels in lysosomes upon USP32KO were assumed to result in altered degradative capacity of the hydrolytic machinery. In order to test this assumption, an indirect read-out by fluorescence microscopy was chosen. The fluorogenic substrate for proteases DQ Red BSA serves as molecular probe. BSA is heavily labeled with a BODIPY dye that is strongly self-quenched. The probe is applied to cells in culture and endocytosed. Eventually, upon digestion of the BSA in the lysosomes by proteases the quenching is relieved and bright fluorescent signal can be detected.

The DQ Red BSA was used in U2OS and MCF7 cells with siRNA mediated USP32 depletion as well as in U2OS CRISPR/Cas9 USP32KO cells. In total, five experiments were performed, images taken and the number of dots quantified. But results could not be reproduced and were inconsistent. Therefore it was decided to stop these experiments without outcome.

Eventually, Rab7 could be verified as USP32 substrate and the function of the USP32-sensitive modification was characterized. Following results and conclusions were presented in this section of the study: 
(I) Rab7 co-immunoprecipitated with USP32, indicating their enzyme-substrate interaction (Figure 3-6 A). (II) Rab7 was ubiquitinated in a USP32-sensitive manner (Figure 3-6 B). (III) This modification did not change Rab7 localization at late endosomal structures (Figure 3-7 A). (IV) But USP32 depletion enhanced the binding of the effector protein RILP suggesting a higher affinity or a higher activity and thus GTP-bound form of Rab7 (Figure 3-7 B). (V) The Rab7 mediated trafficking of Cl-M6PR was promoted upon loss of USP32, faster transport of the receptor to the TGN and general clustering of CI-M6PR at the TGN was observed (Figure 3-8) (VI) Increased cathepsin levels in purified lysosomes from USP32KO cells suggested a more efficient CI-M6PR trafficking and hence hydrolase sorting (Figure 3-9). (VII) Though two more approaches to investigate consequences of altered trafficking were undertaken, neither effects on hydrolase secretion nor enhanced lysosomal degradation capacity could be examined due to technical issues (Figure 3-10 and 3.5.6.6).

\subsubsection{USP32 effect on retromer complex}

The earlier mentioned retromer complex is a highly conserved multimeric complex regulating the recycling of numerous proteins from the endosomes to the TGN or plasma membrane. Its function and dysfunction is linked with several neurodegenerative diseases like Alzheimer's or Parkinson's disease. The retromer complex is assembled of a trimeric cargo recognition complex (CRC) and a part containing sorting nexins. VPS26, VPS29 and VPS35 form the stable $\mathrm{CRC}$ and function as the core of the complex. In the first recruitment of the CRC to endosomal membranes various factors like Rab7 are involved. In the subsequent association of the CRC with defined sorting nexin proteins the cargo selection and trafficking route (to TGN or plasma membrane) is determined (Seaman, 2021; Wang and Bellen, 2015).

The here presented data on Rab7 as a USP32 substrate linked the retromer complex to the DUB. In addition, (Sowa et al., 2009) presented a global DUB interactome study, which identified VPS35 as bona fide interactor of USP32.

These two connections presented VPS35 and the CRC as interesting candidate for further studies in the context of USP32.

\subsubsection{CRC protein levels and composition}

The first experiment on retromer CRC focused on the cellular abundance of CRC components VPS26, VPS29 and VPS35. Therefore U2OS and RPE1 ctrl/NHT or USP32KO cells under basal conditions were lysed and analyzed by immunoblotting with specific antibodies. The expression levels of all three proteins did not change upon loss of USP32 suggesting that USP32 does not regulate CRC levels (Figure 3-11 A).

Next, the compositions of the CRC was studied to answer the question whether USP32. Endogenous VPS35 was immunoprecipitated with a specific antibody from RPE1 NHT or USP32KO cells. Levels of co-immunoprecipitated VPS26A and VPS29 were detected by Western blotting (Figure 3-11 B). VPS35 was nicely enriched from both cell lines in comparable amounts. The interacting levels of VPS26 and VPS29 remained stable upon USP32 depletion in comparison to the control. Thus, a regulation of the CRC by USP32 could not be observed. 
In the next section it was examined whether USP32 depletion affects protein levels of the three CRC components, and the composition of the CRC. Furthermore, the interaction of USP32 and CRC components was characterized.

\subsubsection{USP32-CRC interaction}

In the last experiment to study the connection between USP32 and the retromer complex, the interaction of USP32 and all three complex components was analyzed. Despite the issues of overexpression USP32, a co-immunoprecipitation was performed with exogenous tagged proteins. Either GFP alone as negative control or GFP-USP32 fl was transfected in HEK293 cells with HA-VPS26, Myc-VPS29 and/or HA-VPS35 and a GFP trap IP was done. Samples were subjected to immunoblotting. The proteins were detected by their respective tag with specific antibodies against GFP, HA or Myc. Co-immunoprecipitation of all three CRC components expressed together or individually with USP32 was observed suggesting an indirect or direct interaction (Figure 3-11 C). This finding confirmed the data of (Sowa et al., 2009).

A

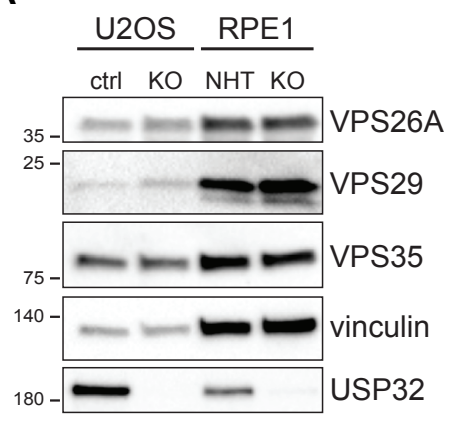

B

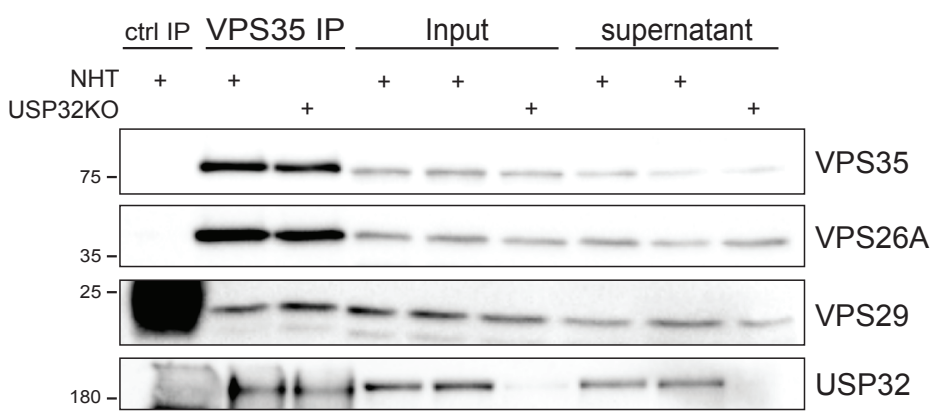

C

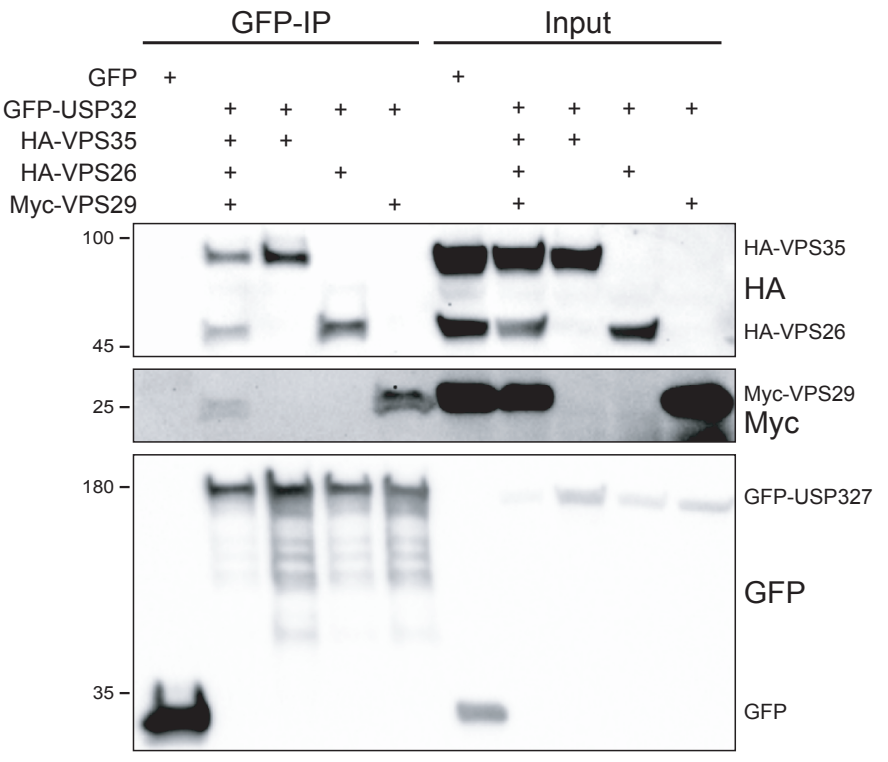

Figure 3-11: USP32 does not directly affect retromer cargo recognition complex. A Abundance of retromer components was stable upon loss of USP32. Lysates of U2OS and RPE1 ctrl/NHT and USP32KO cells were analyzed for retromer components by Western blotting. B Composition of retromer complex did not change in USP32KO cells. Endogenous VPS35 was immunoprecipitated from RPE1 NHT and USP32KO cells with a specific antibody. Protein levels were detected by Western blotting. VPS29 blot shows overexposed signal of control antibody light chain in first lane. C USP32 interacts with all three CRC components. HA-VPS35, HA-VPS26 and Myc-VPS29 coimmunoprecipitated with GFP-USP32 in a GFP-trap IP and were detected by immunoblotting. HA-VPS35, HA-VPS26 and Myc-VPS29 bands in input samples were partially overexposed. 
In summary, the described experiments showed that (I) USP32 did not regulate protein levels of cargo recognition trimer of the retromer complex (Figure 3-11 A). (II) Furthermore, the CRC composition was stable upon USP32 depletion (Figure 3-11 B), and (III) USP32 interaction with VPS35, VPS26, and VPS29 was detected by co-immunoprecipitation (Figure 3-11 C).

\subsection{Characterization of LAMTOR1 as USP32 substrate}

In the previous section the endosomal protein Rab7 was verified as a substrate of USP32 and the function of this deubiquitination was described. The presented data showed not only effects on the endosomal but also on the lysosomal stage unraveling a broader regulation of the dynamic system by USP32. The other identified potential USP32 substrates and the eventual effect on faster or more efficient hydrolase sorting into lysosomes upon depletion of USP32 guided the project to another very interesting hit: LAMTOR1.

The lysosomal Ragulator complex is critical for the regulation of lysosomal metabolic signaling and trafficking. The complex consists of the two tightly packed heterodimers LAMTOR2/LAMTOR3 and LAMTOR4/LAMTOR5, which are wrapped and held together by LAMTOR1. LAMTOR1 tethers the complex to the lysosomal membrane by its $\mathrm{N}$-terminal myristoylation and palmitoylation. Besides a variety of cellular functions, two major functions for Ragulator were described in literature:

The interaction of Ragulator with the heterodimeric RagA/B-RagC/D small GTPases, and the amino acid sensors V-ATPase and SLC38A9 forms a system to transfer the signal to mTORC1. Ragulator was identified as guanine nucleotide exchange factor (GEF) for the Rags (Bar-Peled et al., 2012). Its GEF activity is stimulated by the interaction with the v-ATPase leading to efficient recruitment and activation of $\mathrm{mTORC} 1$ at the lysosomal surface.

Another interaction between Ragulator and BORC regulates anterograde directed movement of lysosomes. This regulation is controlled by growth factor signaling and nutrient availability. Both weakens the interaction of Ragulator and BORC thereby enhancing the outward movement of lysosomes by coupling BORC to kinesin motors (Filipek et al., 2017; Pu et al., 2017).

The next section verifies LAMTOR1 as a USP32 substrate and sheds light on the functions of this modification.

\subsubsection{Interaction of USP32 and LAMTOR1}

Like for Rab7, an interaction of USP32 and its possible substrate LAMTOR1 was suggested. This hypothesis was addressed in the first experiment by performing a co-immunoprecipitation. LAMTOR1-GFP was co-expressed with HA-USP32 in HEK293 cells and immunoprecipitated with GFP-trap beads. Expression of GFP alone in presence of USP32 was used as negative IP control. USP32 binding was examined in the IP sample by using an antibody against its HA tag (Figure 3-12). Though a band for HA-USP32 was detected in the ctrl sample, the intensity of the band in the IP sample was higher (given that precipitated GFP signals were comparable). Thus, interaction of LAMTOR1 and USP32 could be observed. 


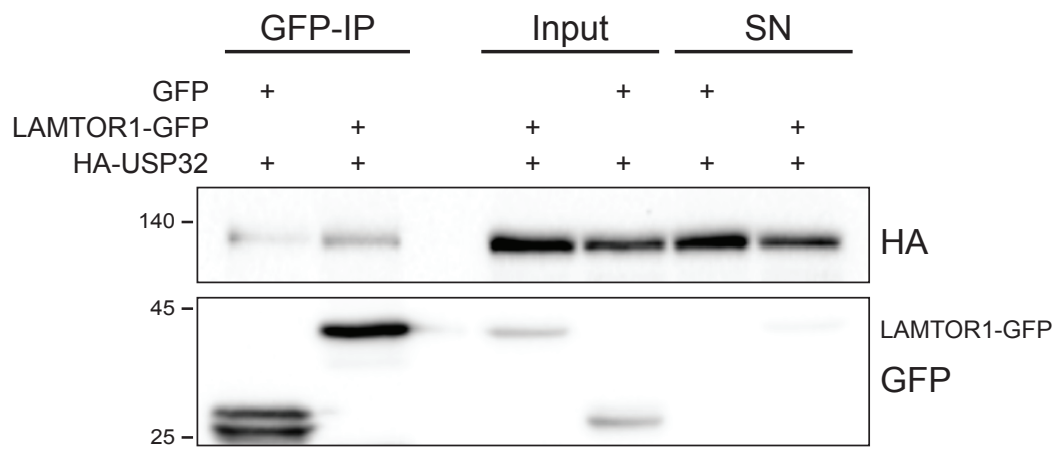

Figure 3-12: USP32 interacts with its possible substrate LAMTOR1. HA-USP32 co-immunoprecipitated with LAMTOR1-GFP in a GFP-trap IP and was detected by immunoblotting.

\subsubsection{USP32-sensitive LAMTOR1 ubiquitination}

Ubiquitination of LAMTOR1 at K20 and K31 has already been reported before, but the function of this modification remained elusive (Wagner et al., 2011). Moreover, a recent study by Sun et al., 2018 described LAMTOR1 ubiquitination by the E3 ligase Ube3a in mice. Ube3a preferentially modified residues $\mathrm{K} 60$ and $\mathrm{K} 103 / 104$ resulting in its proteasomal degradation. Lysosomal localization of LAMTOR1/Ragulator, and mTORC1 activity was increased in the absence of Ube3a.

In the course of this study, the USP32-dependent ubiquitination of LAMTOR1 and its cellular function was investigated. A label free mass spectrometry approach was chosen to verify the identified USP32-sensitive ubiquitination of LAMTOR1 in general and especially at the already detected residue K20 (see 3.4). For this, RPE1 NHT and USP32KO cells were transfected with LAMTOR1-GFP. A GFP-trap IP was performed. The samples were subjected to on-bead insolution digest and subsequent SDB-RPS STAGE tip purification and enrichment. Afterwards the samples were analyzed by LC-MS/MS analysis for the LAMTOR1 ubiquitin modification in collaboration with Georg Tascher (Institute of Biochemistry II, Goethe University Frankfurt) (detailed protocol see 6.2.10.4).

General protein intensity was determined as equal for both RPE1 NHT and USP32KO samples serving as control for comparable enrichment. LAMTOR1 lysine residues at positions 20, 31, 60, and 104 were identified to be ubiquitinated. K20 was the only significantly enriched site upon USP32KO ( $p$-value $<0.05$ ). But K31 and especially K60 showed also increased log2 USP32KO/NHT ratios (Figure 3-13 A). This implied that also ubiquitin signals from other residues than K20 were cleaved by USP32, but to a smaller extend. Notably, the intensity of K60 diGly site was highest for all four identified sites consistent to the data of (Sun et al., 2018).

A complementary verification of LAMTOR1 ubiquitination in an USP32-dependent manner was approached by His-ubi pulldown. His-tagged ubiquitin was co-expressed with different mutants of either LAMTOR1-GFP or HA-USP32 in HEK293 cells. His-ubi was enriched with Ni-NTA beads and samples were subjected to immunoblotting. The modification of LAMTOR1 was detected with a specific antibody against the endogenous protein, and was visible as high molecular smear on a Western blot (Figure 3-13 B). In cells co-expressing wildtype (wt) LAMTOR1 with either wt catalytic active USP32 or catalytic inactive C743S mutant of USP32 the modification of 
LAMTOR1 was increased when USP32 was catalytic inactive. Comparing co-expression of USP32 wt or C743S together with LAMTOR1 K20R mutant produced the same pattern: LAMTOR1 modification was increased in the presence of mutant USP32 (C743S). A myristoylation-deficient mutant of LAMTOR1 (G2A) nearly completely lost its ubiquitination. These findings suggest that (I) LAMTOR1 is modified by USP32. (II) USP32 deubiquitinates LAMTOR1 not exclusively on K20 but also other residues confirming the previous mass spectrometry experiment on LAMTOR1 ubiquitination. (III) Lysosomal localization, dependent on its N-terminal myristoylation, is critical for LAMTOR1 ubiquitination.

The signal of amino acid sensors is transferred to Ragulator in response of amino acid availability. Hence, it was tested if LAMTOR1 is ubiquitinated in accordance with amino acid levels.

Since overexpression of His-ubiquitin could compete with endogenous ubiquitin and result in artefactual ubiquitination patterns, in another experiment to enrich ubiquitinated proteins Tandem Ubiquitin Binding Entities (TUBEs) were used to address this question. TUBEs are polymerized ubiquitin associated domains that bind to poly-ubiquitin chains with high affinity. Conjugation to beads enables efficient and fast purification and enrichment (Hjerpe et al., 2009; Mattern et al., 2019).

Lysates from untreated or starved RPE1 NHT or USP32KO cells were incubated with magnetic TUBE1 agarose beads. The enriched samples were prepared for SDS-PAGE and subsequently immunoblotting was applied. LAMTOR1 modification was detected with an antibody against the endogenous protein (Figure 3-13 C). LAMTOR1 smear was slightly increased in USP32KO samples under basal conditions. Moreover, the abundance of unmodified LAMTOR1 was decreased. Starvation with EBSS for $2 \mathrm{~h}$ showed the same outcome of less unmodified LAMTOR1 and slightly darker LAMTOR1 smear at higher molecular weights upon USP32 depletion. This experiment confirmed again the USP32-sensitive modification of LAMTOR1 and indicated this modification is independent of amino acid availability. 
A
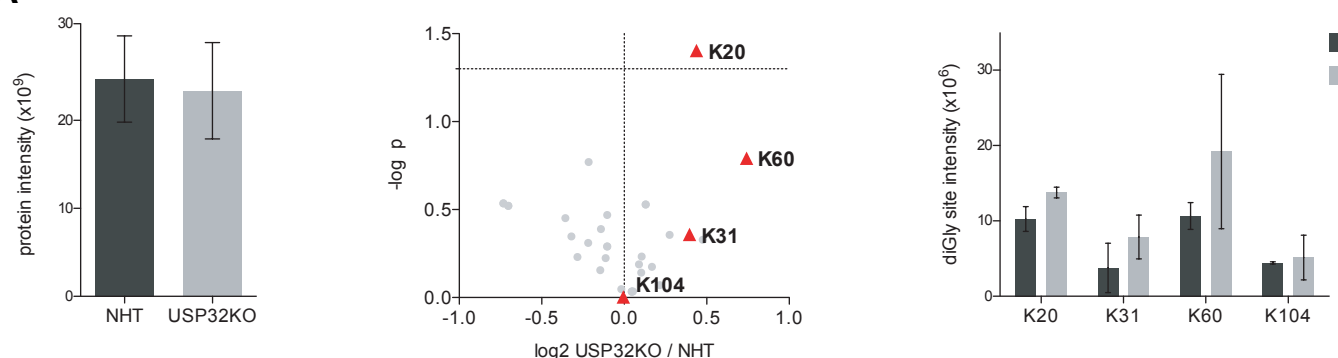

$\mathrm{NHT}$

USP32KO
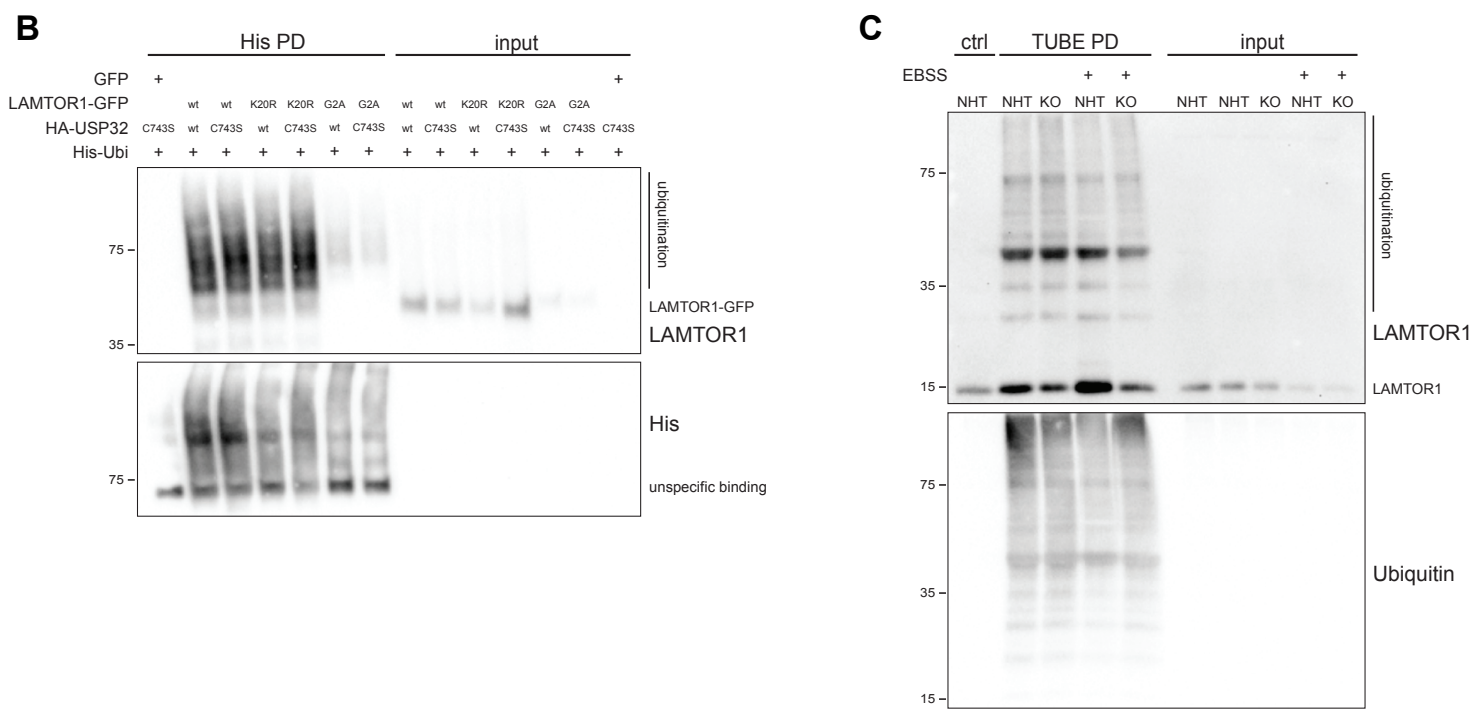

Figure 3-13: LAMTOR1 ubiquitination is USP32-sensitive. A LAMTOR1 ubiquitination at position 20 was significantly enhanced in USP32KO cells. LAMTOR1-GFP was immunoprecipitated from RPE1 NHT or USP32KO cells and ubiquitination status was analyzed by LC-MS/MS. B LAMTOR1 ubiquitination was dependent on the catalytic activity of USP32. His-ubiquitin tagged proteins were pulled down from HEK cells co-expressing LAMTOR1-GFP wt, K20R or G2A mutant with HA-USP32 active wt or inactive C743S mutant and probed against LAMTOR1. C LAMTOR1 ubiquitination was increased in absence of USP32 independent of amino acid availability. Ubiquitinated proteins were enriched from untreated or $2 \mathrm{~h}$ EBSS starved RPE1 NHT and USP32KO cells using TUBEs conjugated to magnetic beads. Modification was detected by Western blotting with a LAMTOR1 specific antibody.

\subsubsection{LAMTOR1 localization at lysosomes}

Sanders et al. 2019 showed that LAMTOR1 localization is mediated by dynamic addition of palmitoyl and myristoyl groups. Not only lipidation but also ubiquitination was reported to regulate LAMTOR1 (and Ragulator) localization to lysosomes (Sun et al., 2018). In line with these data, IF microscopy was used to examine whether increased LAMTOR1 ubiquitination caused by USP32 knockout changes its position at lysosomes.

LAMTOR1 and the lysosomal marker LAMP2 were co-stained in RPE1 as well as U2OS NHT/ctrl and USP32KO cells under basal or amino acid starved conditions. The overall correlation of the two signals was quantified over the acquired confocal microscopy images. The co-localization of LAMTOR1 and LAMP2 was presented by the Pearson's correlation coefficient (Figure 3-14). In untreated and starved cells LAMTOR1 localization at LAMP2 positive structures was decreased upon USP32 depletion suggesting that USP32-sensitive ubiquitination is regulating LAMTOR1 positioning in an amino acid-independent manner. 
A
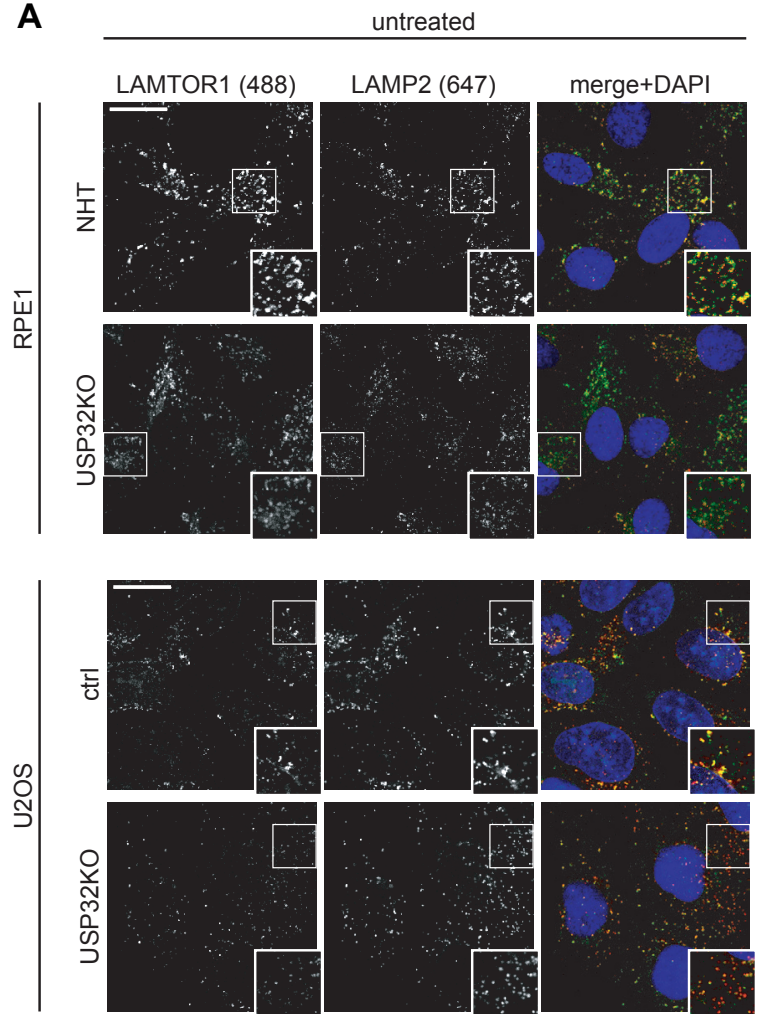

B

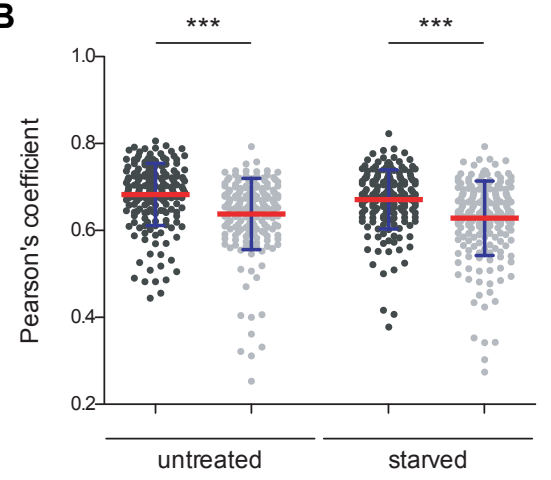

RPE1
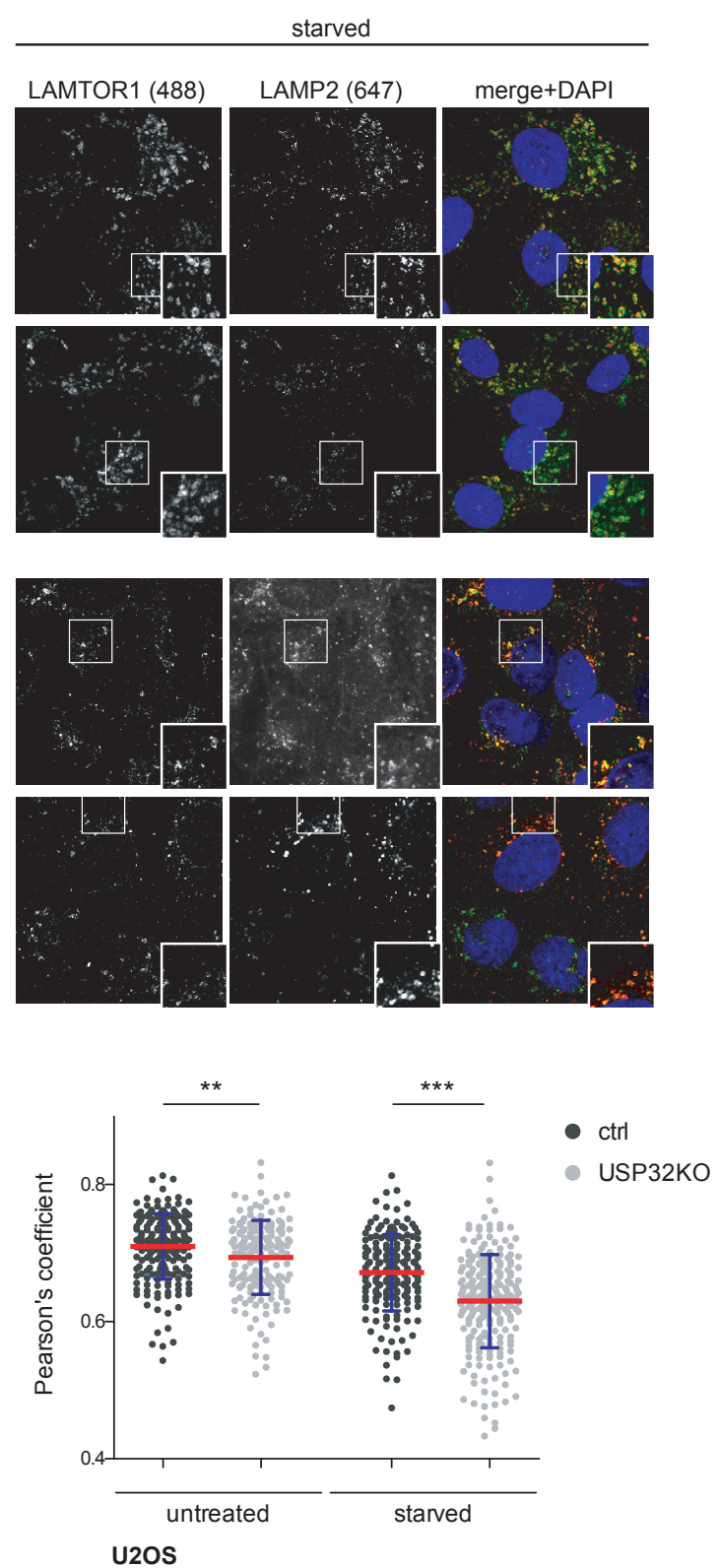

Figure 3-14: Lysosomal LAMTOR1 localization is partially lost in USP32KO cells. A Representative images of LAMTOR1 and LAMP2 co-staining in untreated and $2 \mathrm{~h}$ EBSS starved RPE1 and U2OS NHT/ctrl and USP32KO. Scale bar $=20 \mu \mathrm{m}$. B Quantification of LAMTOR1 co-localization to LAMP2 positive structures shown as LAMTOR1-LAMP2 Pearson's co-localization coefficient (per cell) with indicated mean $\pm \mathrm{SD}, * * \mathrm{p}<0.01, * * * \mathrm{p}<0.0001$ in a two-tailed, unpaired Student's t test between the indicated conditions.

\subsubsection{Lysosome positioning}

Ragulator functions as mediator of lysosomal transport to the periphery of cells under basal conditions. LAMTOR1 knockdown was reported to shift lysosomes toward the cell periphery (Pu et al., 2017). After identification of USP32 regulated LAMTOR1 localization the next question was if the general lysosomal positioning was altered. For this, the LAMP2 images of the previous experiment were used to quantify the distributions of dots in the cell. Every cell was dissected in two bins around the nucleus (DAPI) and the mean intensity within the ring was measured.

In both RPE1 NHT and U2OS ctrl cells, the intensity in bin 1 slightly increased upon starvation describing the lysosome clustering during amino acid deprivation. This tendency was not 
observed in USP32KO cells (Figure 3-15). Nevertheless, all quantified changes were not statistically significant comparing either $\mathrm{NHT} / \mathrm{ctrl}$ to USP32KO or untreated to starved conditions.
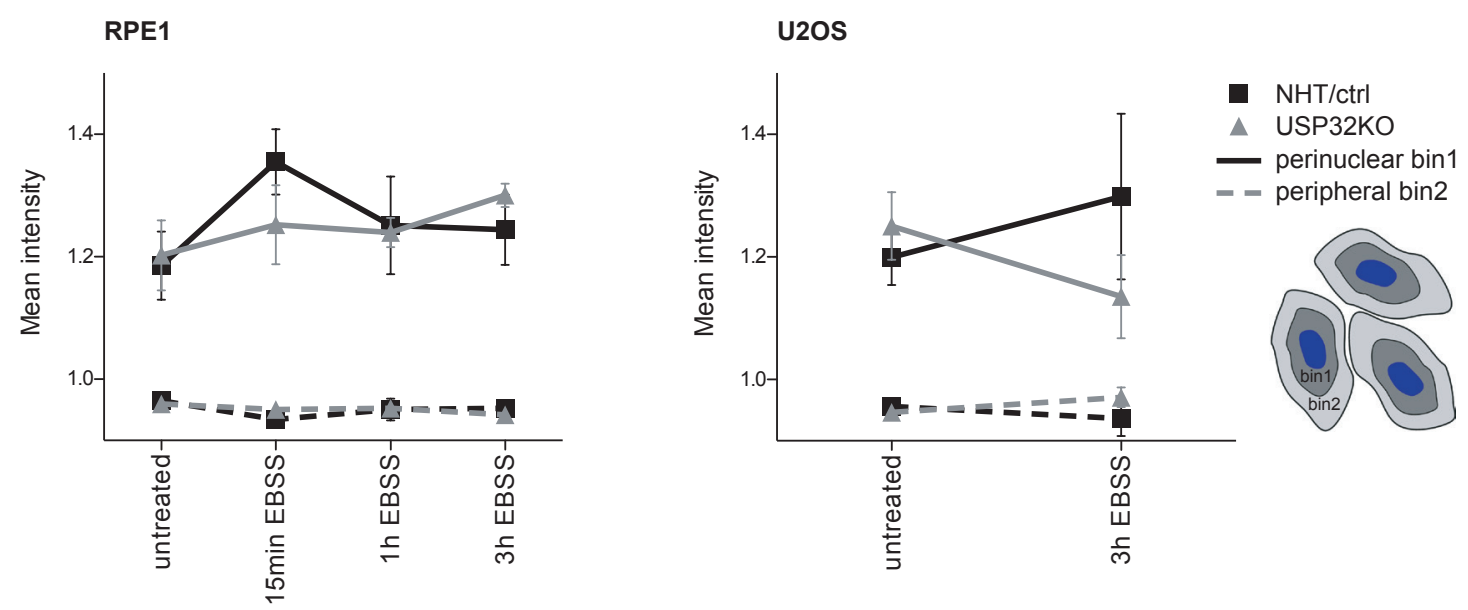

Figure 3-15: USP32 does not regulate lysosome distribution in cells. Quantification of LAMP2 intensity distribution in the perinuclear and peripheral bin of cells presented as mean $\pm S D$. The cartoon describes the segmentation of cells in bin1 (dark grey) and bin2 (light grey) around the nucleus (blue) for quantification.

Taken together, LAMTOR1 localization at lysosomes but not overall lysosome positioning is altered in USP32KO cells.

\subsubsection{LAMTOR1 interactome}

Showing that the lysosomal transport is not regulated by USP32 the project focused in the following part on the second major function of Ragulator: regulation of mTORC1 signaling.

A number of upstream players to relay amino acid signals to mTORC1 were identified. These signaling complexes assemble on the membrane of lysosomes. In brief, the lysosomal v-ATPase senses amino acid levels and stimulates the GEF activity of Ragulator, which scaffolds and activates the Rag GTPases (Bar-Peled et al., 2012). In addition, the amino acid transporter SLC38A9 acts as GEF for Rags, as well (Shen and Sabatini, 2018). The thereby increased affinity of Rags for mTORC1 brings the kinase to the lysosomal surface, the site of its activation by RHEB. GATOR1 and folliculin terminate the signaling by their GAP function for Rags (Bar-Peled et al., 2013; Shen and Sabatini, 2018; Tsun et al., 2013). In summary, following complexes (and their components/subunits) orchestrate mTORC1 activation:

Table 3-2: Proteins and complexes involved in the regulation of mTORC1 activity at lysosomes

\begin{tabular}{l|l}
$\begin{array}{l}\text { Lysosomal v-ATPase } \\
\text { Ragulator } \\
\text { SLC38A9 }\end{array}$ & V0 and V1 subunits \\
Rags & LAMTOR1-5 \\
mTORC1 & RagA/B, RagC/D \\
RHEB & \\
GATOR1 & DEPDC5, Nprl2, and Nprl3 \\
Folliculin & +FNIP2
\end{tabular}


The interaction of the complexes is not only regulated by amino acids but also posttranslational modifications have been reported to coordinate interactions and activity. Sun et al., 2018 described Ube3a-mediated LAMTOR1 ubiquitination affecting the interaction with RagA. Therefore, it was hypothesized that interaction with one or more of the above listed proteins and complexes could be affected by USP32-sensitive LAMTOR1 ubiquitination.

To investigate this assumption a label free mass spectrometry approach investigating the LAMTOR1 interactome was applied. LAMTOR1-GFP was transiently expressed in RPE1 NHT or USP32KO cells. Afterwards, GFP-trap IP was performed. An on-bead in-solution digest and subsequent SDB-RPS STAGE tip purification and enrichment was performed. The samples were analyzed by LC-MS/MS analysis for the co-immunoprecipitated proteins in collaboration with Georg Tascher (Institute of Biochemistry II, Goethe University Frankfurt) (detailed protocol see 6.2.10.4).

The volcano plot for interactome 1 in Figure 3-16 A depicts the identified peptides with corresponding fold change (expressed in log2 ratios) and p-values (expressed in -log). Comparison was done between USP32KO (heavy) and $\mathrm{NHT}$ (light) samples. Identified proteins with log2 ratio $\leq-0.6$ or $\geq 0.6$ and $p$-value $\geq 0.05$ were considered as significantly down- or upregulated upon USP32 depletion.

LAMTOR2-5 as well as RHEB were detected with no changes in their interaction. No proteins of interest involved in $\mathrm{mTORC} 1$ regulation, showed enriched binding with LAMTOR1. But very strikingly, two subunits of the lysosomal V-ATPase, ATP6V0C and ATP6V1A, were identified with decreased interaction with LAMTOR1 when USP32 was knocked out.

In a second mass spectrometry experiment (interactome 2) it was investigated whether the changes of the LAMTOR1 interactome upon loss of USP32 respond to amino acid availability. The same experimental setup was chosen. Besides the untreated samples a short-term starved ( $3 \mathrm{~h}$ EBSS) sample as well as an overnight starved and re-fed sample ( $16 \mathrm{~h}$ EBSS followed by 30 min full DMEM) was included. The LC-MS/MS analysis confirmed the previous result of decreased interaction of LAMTOR1 and two other subunits of the V-ATPase, ATPVOA2 and ATP6AP1 (Figure 3-16). The additional treatment resulted in no other relevant changes of the interactome neither comparing different treatments in the same cell line nor comparing USP32KO and NHT upon the same treatment.

A interactome 1

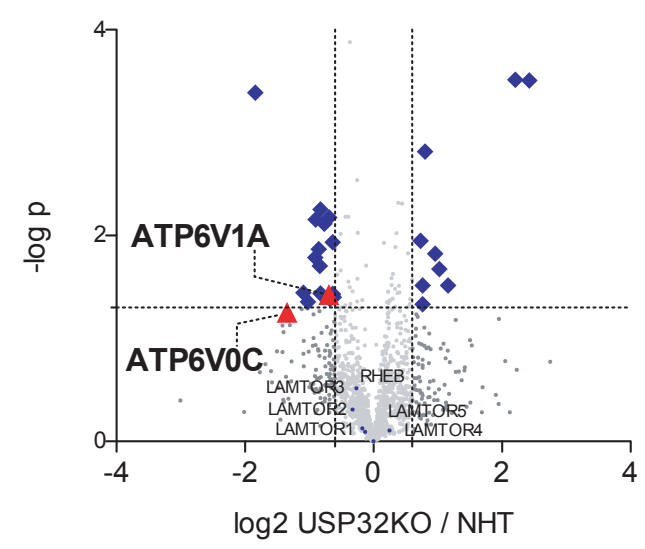

interactome 2

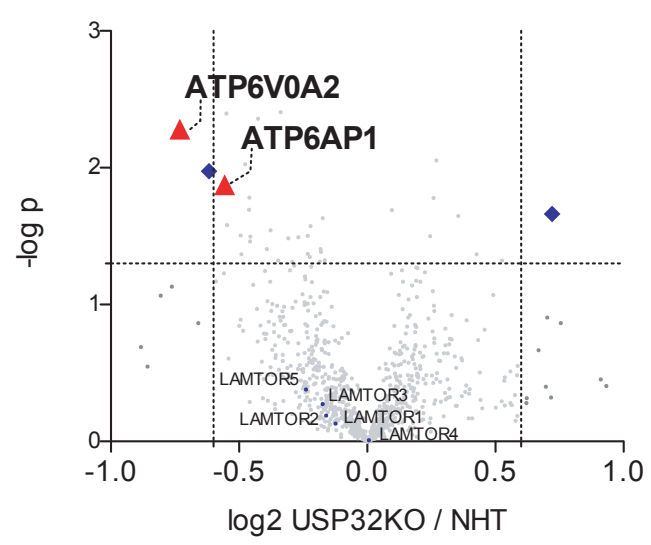


B

\begin{tabular}{l|cc}
\multicolumn{3}{c}{ interactome 1} \\
Protein & log2 (KO/NHT) & $-\log (\mathrm{p})$ \\
\hline ERLIN2 & 2,42 & 3,51 \\
DPM1 & 2,21 & 3,51 \\
ALDH16A1 & 1,16 & 1,52 \\
MRPL13 & 1,03 & 1,68 \\
HK2 & 0,96 & 1,82 \\
HSD17B11 & 0,80 & 2,82 \\
TIMM44 & 0,77 & 1,51 \\
MEMO1 & 0,76 & 1,33 \\
CSK & 0,73 & 1,95 \\
\hline RPS21 & $-0,62$ & 1,40 \\
G3BP1 & $-0,63$ & 1,43 \\
TAGLN & $-0,64$ & 1,93 \\
CALM1/2/3 & $-0,64$ & 1,42 \\
ARHGAP1 & $-0,68$ & 1,38 \\
DUT & $-0,69$ & 1,40 \\
YKT6 & $-0,69$ & 2,18 \\
ATP6V1A & $-0,69$ & 1,43 \\
SNRPA/B & $-0,77$ & 2,11 \\
TPM4 & $-0,82$ & 1,44 \\
RPS28 & $-0,82$ & 2,25 \\
BASP1 & $-0,83$ & 1,71 \\
MAP4 & $-0,85$ & 1,87 \\
CALD1 & $-0,90$ & 2,16 \\
SERBP1 & $-0,91$ & 1,79 \\
RAC1/2/3 & $-1,02$ & 1,36 \\
CAP1 & $-1,09$ & 1,45 \\
ATP6V0C & $-1,34$ & 1,25 \\
HDGF & $-1,84$ & 3,39 \\
& &
\end{tabular}

\begin{tabular}{l|cc}
\multicolumn{3}{c}{ interactome 2} \\
Protein & $\log 2(\mathrm{KO} / \mathrm{NHT})$ & $-\log (\mathrm{p})$ \\
\hline BANF1 & 0,72 & 1,66 \\
\hline ATP6AP1 & $-0,56$ & 1,88 \\
CYC1 & $-0,62$ & 1,97 \\
ATP6V0A2 & $-0,73$ & 2,28
\end{tabular}

Figure 3-16: Interaction of LAMTOR1 and lysosomal V-ATPase subunits is weaker upon USP32 depletion. A Volcano plots of both interactome 1 and interactome 2 depicting the identified peptides with corresponding fold change (expressed in log2 ratios) and p-values (expressed in -log). Comparison was done between USP32KO and NHT. Identified proteins with log2 ratio $\leq-0.6$ or $\geq 0.6$ and $p$-value $\geq 0.05$ were considered as significantly down- or upregulated upon USP32 depletion and labeled in blue. Lysosomal v-ATPase subunits as interesting hits were labeled in red. Each data set was obtained from one GFP-trap IP sample set with three technical replications. B Table listing all significantly up- or downregulated interacting proteins with their respective $\log 2$ ratios and $-\log (p)$-values. Interesting proteins were marked in bold.

\subsubsection{Effect on mTORC1}

Several publications characterize how the amino acid signal regulates the v-ATPase-RagulatorRags-mTORC1 relation on molecular and structural basis (Bar-Peled et al., 2012; de Araujo et al., 2017; Yonehara et al., 2017; Zoncu et al., 2011). Association of the v-ATPase with Ragulator under nutrient-rich conditions stimulates Ragulator's GEF activity. The Rag GTPases switch from their inactive to the active form thereby weakening the interaction to Ragulator and enhancing the affinity for mTORC1. Though it is not completely understood how amino acids regulate the $\mathrm{V}$-ATPase-Ragulator interaction, it is supposed that under starved conditions Ragulator has a lower GEF activity resulting in inactive Rags and inactivation of mTORC1. These 
consecutive interactions and stimulations are crucial for mTORC1 recruitment to the lysosomal surface and activation, and its downstream signaling.

Consequentially, this study investigated the localization of mTORC1 and its activity in dependence of USP32.

\subsubsection{1 mTORC1 localization}

To test whether mTORC1 recruitment and localization to the lysosomal surface was impaired the localization of its component mTOR was examined by immunofluorescence microscopy in RPE1 NHT and USP32KO cells. Amino acid availability was also investigated by additional starvation with EBSS. mTOR was stained together with LAMP2. Their co-localization was quantified over the entire image and presented as Pearson's correlation coefficient. Figure 3-17 shows representative images and the quantification.

Indeed, upon depletion of USP32 the localization of mTOR relative to LAMP2 decreased. The same but no further effect was observed after EBSS treatment. It was proposed that USP32 regulates $\mathrm{mTORC} 1$ lysosomal localization in an amino acid-independent way.

A
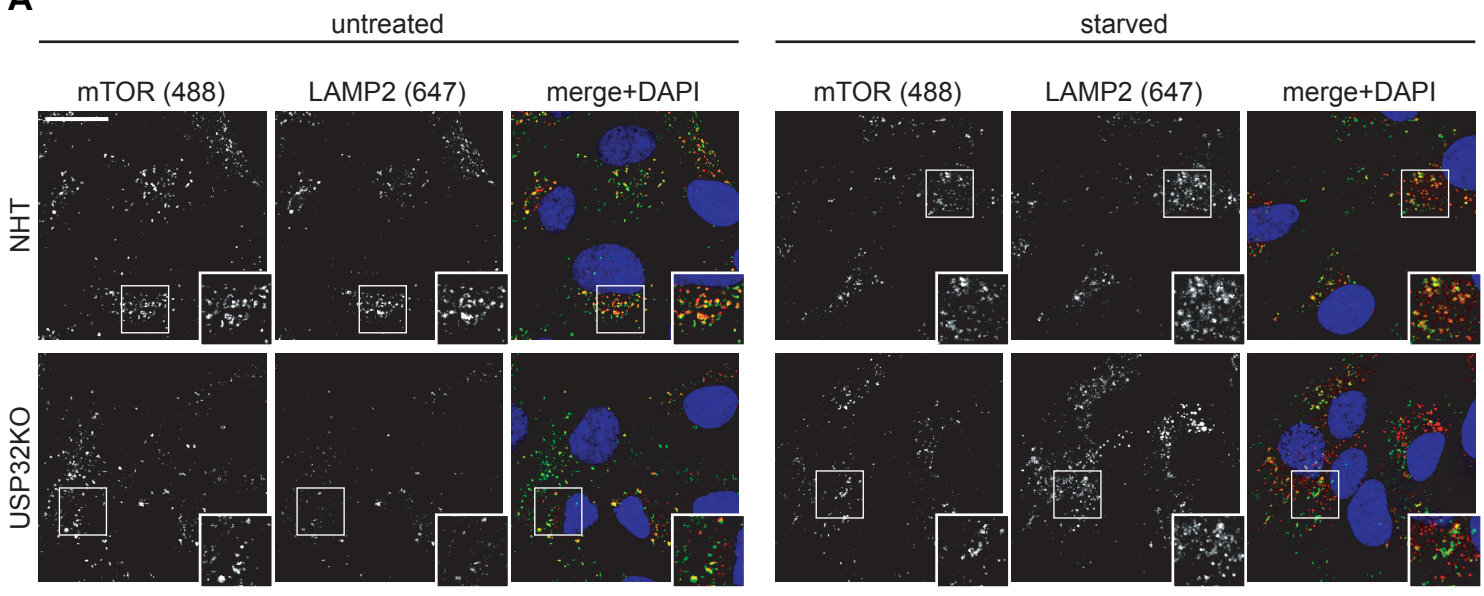

B

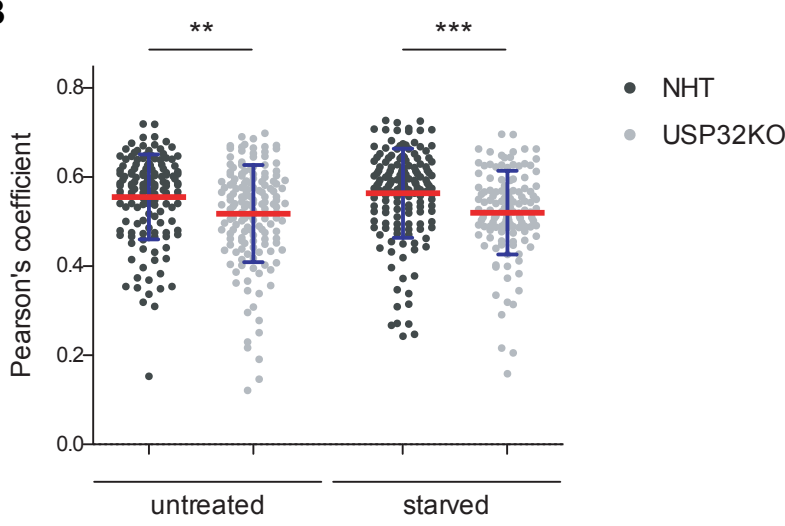

Figure 3-17: Lysosomal mTOR localization is partially lost in USP32KO cells. A Representative images of mTOR and LAMP2 co-staining in untreated and $2 \mathrm{~h}$ EBSS starved RPE1 NHT and USP32KO. Scale bar $=20 \mu \mathrm{m}$. B Quantification of mTOR co-localization to LAMP2 positive structures shown as mTOR-LAMP2 Pearson's colocalization coefficient (per cell) with indicated mean $\pm S D, * * p<0.01, * * * p<0.0001$ in a two-tailed, unpaired Student's $t$ test between the indicated conditions. 


\subsubsection{2 mTORC1 activity}

A consequence of less mTORC1 at the lysosomal membrane is its lack of activation and therefore decreased serine phosphorylation of substrates. The next experiment addressed the question if USP32KO and the altered mTOR localization result in decreased mTORC1 activity. RPE1 NHT and USP32KO cells were starved with EBSS overnight and then re-fed with full medium. The subsequent re-activation of mTORC1 within $1 \mathrm{~h}$ was observed by the phosphorylation of direct downstream substrates. Antibodies specific for (I) ULK1/pULK1 S757, (II) WIPI2/pWIPI2 S413 and (III) AMBRA1/pAMBRA1 S52 were used for immunoblotting (Figure 3-18). The total protein levels of all three substrates were comparable after starvation and re-activation in NHT and USP32KO. But importantly, the levels of phosphorylated proteins were decreased after the indicated time points in full medium. The result of this experiment suggested that re-activation of mTORC1 was impaired upon loss of USP32.
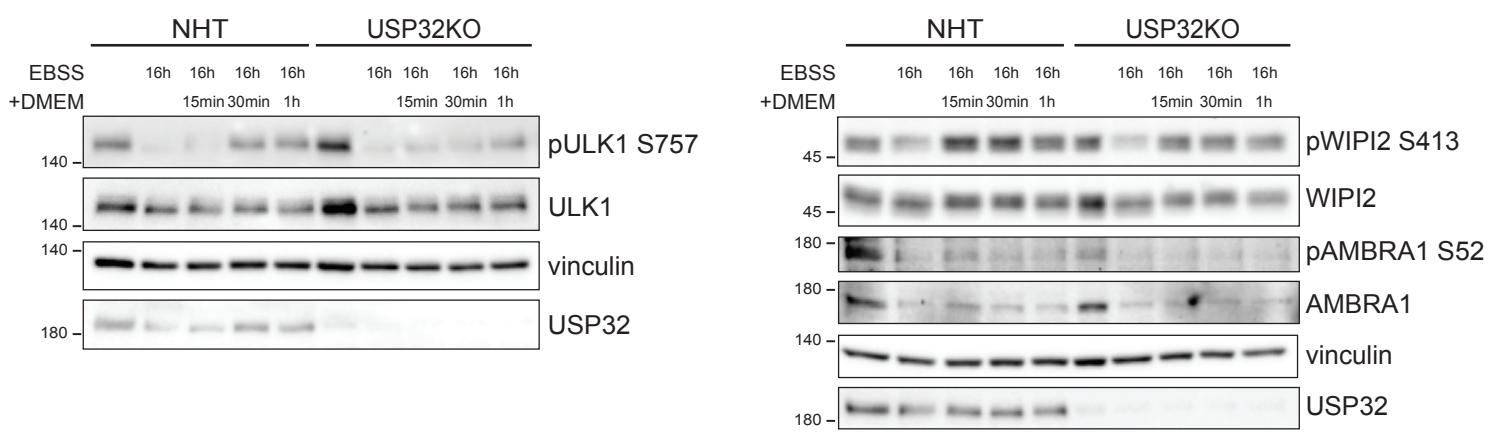

Figure 3-18: Phosphorylation of $\mathrm{mTORC} 1$ substrates is decreased after reactivation in USP32KO cells. RPE1 NHT or USP32KO cells were starved for $16 \mathrm{~h}$ with EBSS and reactivated again with full DMEM for the indicated time points and protein levels were detected by Western blotting using phosphorylation specific antibodies.

Summarizing the third section of this work, LAMTOR1 could be verified as USP32 substrate and the function of the USP32-sensitive modification was characterized. Following results and conclusions were presented:

(I) LAMTOR1 co-immunoprecipitated with USP32 supporting their enzyme-substrate interaction (Figure 3-12). (II) LAMTOR1 was ubiquitinated in an USP32-sensitive but amino acidindependent manner. Though loss of USP32 only resulted in a significant enrichment of ubiquitination at $\mathrm{K} 20$, also other LAMTOR1 residues were expected to be deubiquitinated by USP32 (Figure 3-13). (III) Lysosomal localization of LAMTOR1 was decreased in USP32KO situation independently of amino acid availability (Figure 3-14). (IV) USP32 depletion downregulated the interaction of LAMTOR1 and subunits of the lysosomal V-ATPase under basal conditions (Figure 3-16). (V) As a consequence of wakened LAMTOR1-V-ATPase interaction and possible lower Ragulator GEF activity, mTOR localized less to lysosomal structures upon USP32 loss. Furthermore, mTORC1 was detected with reduced activity in absence of USP32 (Figure 3-17 and Figure 3-18). 


\subsection{Increased autophagy}

During the course of this work Rab7 and LAMTOR1 were characterized as substrates of USP32. Both proteins play an important role in regulation of endosomal-lysosomal trafficking and intracellular signaling.

Besides its role at late endosomes and lysosomes Rab7 was also reported to be essential for proper autophagy progression by regulation of the bidirectional transport of autophagosomes on microtubules, and by mediating the fusion of autophagosomes and lysosomes (1.1.5).

Decreased activity of mTORC1 induces autophagy in different layers, e.g. activation of direct downstream complexes like ULK1 (1.2.3) or enhanced transcription of autophagy genes by TFEB (1.2.2).

Furthermore, many DUBs have been described to be involved in all different steps of autophagy (Magraoui et al., 2015). With the data of this study and the published background, an eventual effect of USP32 depletion on autophagy was examined by immunofluorescence microscopy and immunoblot analysis of the autophagosome marker LC3 and its lipidation.

For microscopy, LC3A/B was stained in RPE1 and U2OS NHT/ctrl and USP32KO cells under basal and starved conditions. When LC3 accumulates in autophagosomes it forms dot structures. The number of dots was quantified as a direct read-out for the autophagy process. Without treatment no significant difference between control and USP32KO cells was quantified. When EBSS starvation was applied for $2 \mathrm{~h}$ an increase in the number of dots per cells appeared upon loss of USP32 (Figure 3-19). 


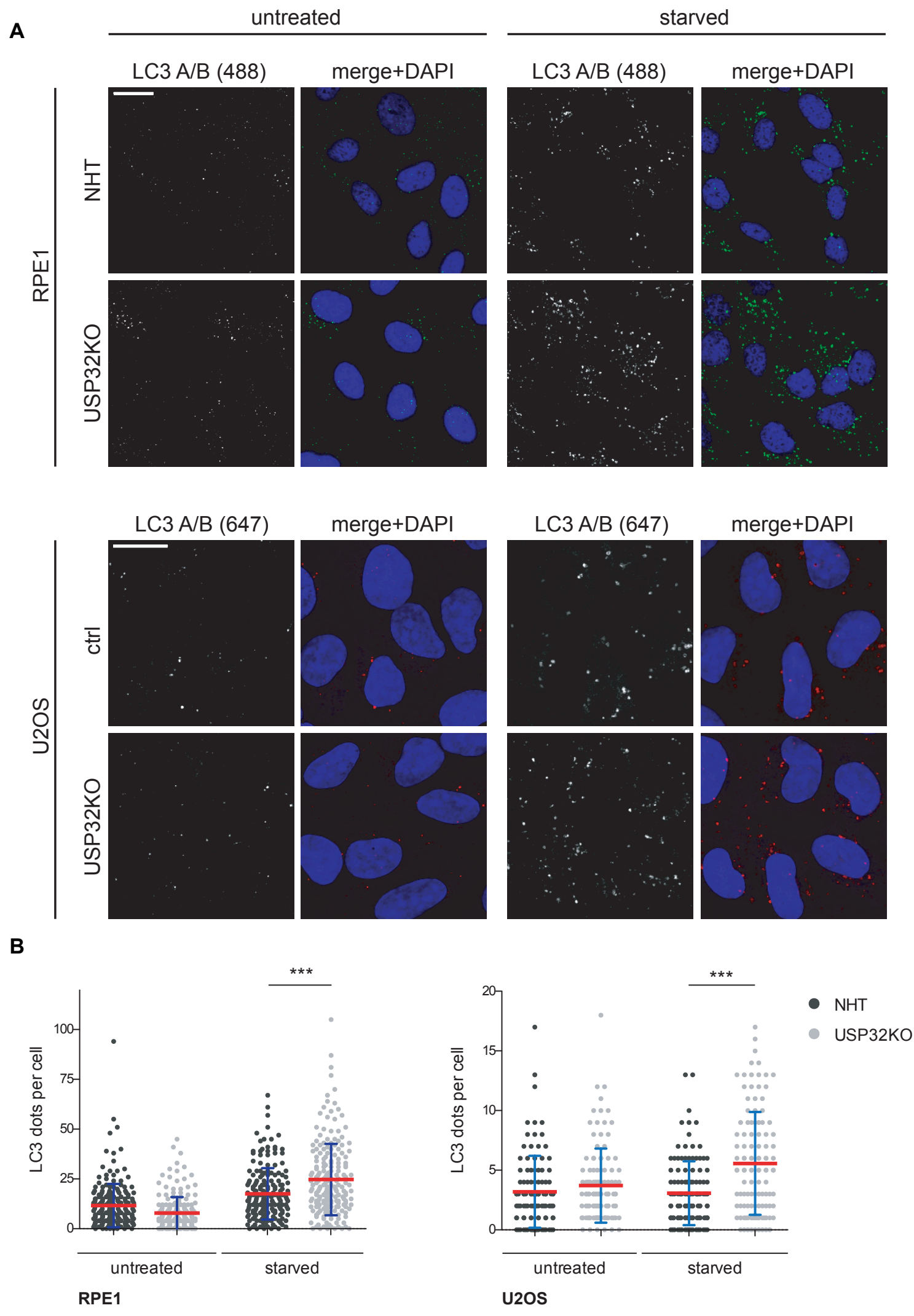

Figure 3-19: USP32-depleted cells show more LC3 puncta upon starvation. A Representative images of LC3A/B staining in untreated and $2 \mathrm{~h}$ EBSS starved RPE1 and U2OS NHT/ctrl and USP32KO cells. Scale bar $=20 \mu \mathrm{m}$. B Quantification of number of LC3 positive dots shown as number of dots per cell with indicated mean \pm SD, $* * * p<0.0001$ in a two-tailed, unpaired Student's $t$ test between the indicated conditions. 
Though LC3 is present in cells in basal levels, upon autophagy induction its transcription is enhanced and after lipidation the protein is conjugated to inner and outer membranes of the growing phagophore. After fusion of autophagosomes and lysosomes LC3 on the inside is degraded along with the cargo, LC3 on the outside dissociates from the membrane (Glick et al., 2010).

The used antibody recognizes both the unlipidated (LC3-I) and lipidated (LC3-II) form of LC3. Thus, an increase in LC3 dot number could correspond to (I) elevated LC3 transcription, (II) enhanced autophagy induction or (III) impaired lysosomal degradation.

To evaluate autophagic flux in more detail, a Western blot analysis approach was chosen. Lipidation of LC3 leads to different running behavior on a SDS-PAGE and can be detected on a subsequent immunoblot at lower molecular weight as the unlipidated species. Autophagic flux and therefore LC3 lipidation can be induced by amino acid starvation. The inhibition of the lysosomal degradation with drugs (Bafilomycin A1 is commonly used) increases the LC3-II levels even further. By observing the LC3-II species upon both treatments alone or in combination it is possible to investigate the effect of protein depletion on the autophagic flux: higher LC3-II levels upon starvation only can correspond to elevated autophagy induction, or lower degradation efficiency. Adding Bafilomycin A1 (BafA1) can resolve the two possibilities. In case LC3-II accumulates even more, a faster flux would be induced by protein depletion. On the other hand, if the LC3-II levels of control and depleted samples are comparable, the late degradation would be impaired.

A complementary immunoblot approach was applied to dissect the described possibilities for increased LC3 in the IF experiment. Autophagy was induced in cells with EBSS starvation. In addition, treatment with BafA1 was used to inhibit the lysosomal degradation. Repeated experiments with CRISPR KO cells showed no differences between ctrl and USP32KO situation (data not shown).

Since cells can adapt over time to a knockout of a gene with compensatory mechanisms, a short-term USP32 depletion with specific siRNA was used in additional experiments. Specific siRNA against USP32 (siUSP32) and non-targeting control siRNA (siNT) were transfected in RPE1 and U2OS cells. $48 \mathrm{~h}$ after transfection the cells were treated and eventually lysed. The lysates were subjected to immunoblotting. LC3-I levels remained comparable between siNT and siUSP32 transfected cells indicating no influence on LC3 transcription. In both cell lines an increase of lipidated LC3A/B was detected upon USP32 knockdown under basal conditions, and after short 15 min as well as longer $2 \mathrm{~h}$ starvation. Addition of BafA1 in RPE1 cells increased LC3 levels slightly, which was not observed in U2OS cells (Figure 3-20). 

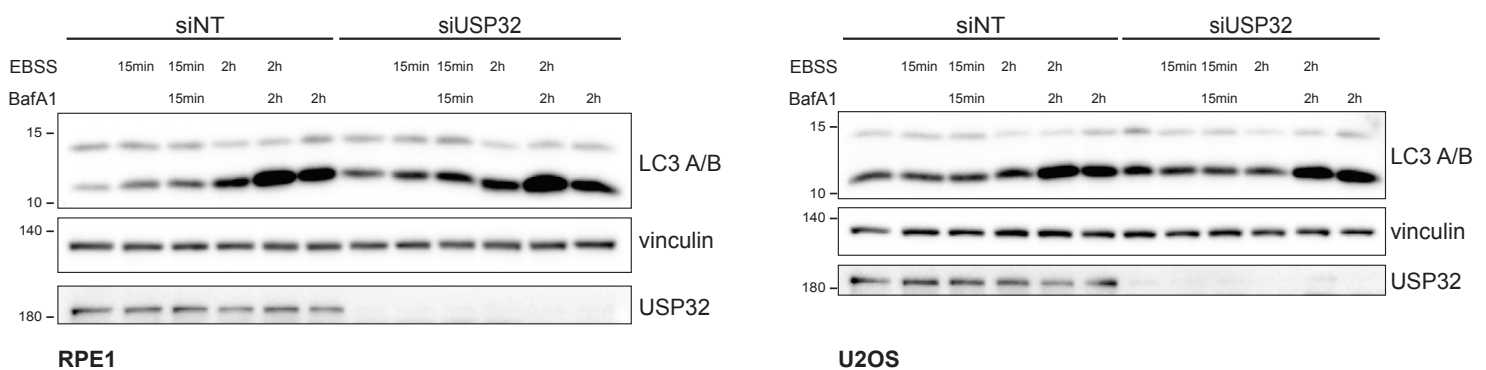

Figure 3-20: USP32 depletion impairs autophagic flux. RPE1 and U2OS cells were transfected with either nontargeting control siRNA (siNT) or siRNA targeting USP32 (siUSP32). After starvation with EBSS and/or treatment with $200 \mathrm{nM}$ Bafilomycin A1 for the indicated time points LC3A/B levels were detected by Western blotting.

Eventually, deubiquitination of Rab7 and LAMTOR1 by USP32 and the resulting trafficking and mTORC1 phenotypes negatively regulated autophagy. Enriched lipidated LC3 levels in RPE1 USP32KO cells upon starvation and inhibition of lysosomal degradation suggested rather induction of autophagy upon USP32 depletion. This phenotype was not observed in U2OS cells leading to the conclusion that USP32 functions at different stages of the endosomal and lysosomal system with an overall influence on early and late autophagy steps. 


\section{Hypothesis model of USP32 function}

After the basic characterization of USP32 and identification of substrates in the endosomal and lysosomal system, two candidates were further characterized. Based on the acquired data presented in the previous section, following models were hypothesized for the cellular function of USP32.

\subsection{USP32 regulates non-proteolytic Rab7 ubiquitination and protein trafficking in the endosomal-lysosomal system}

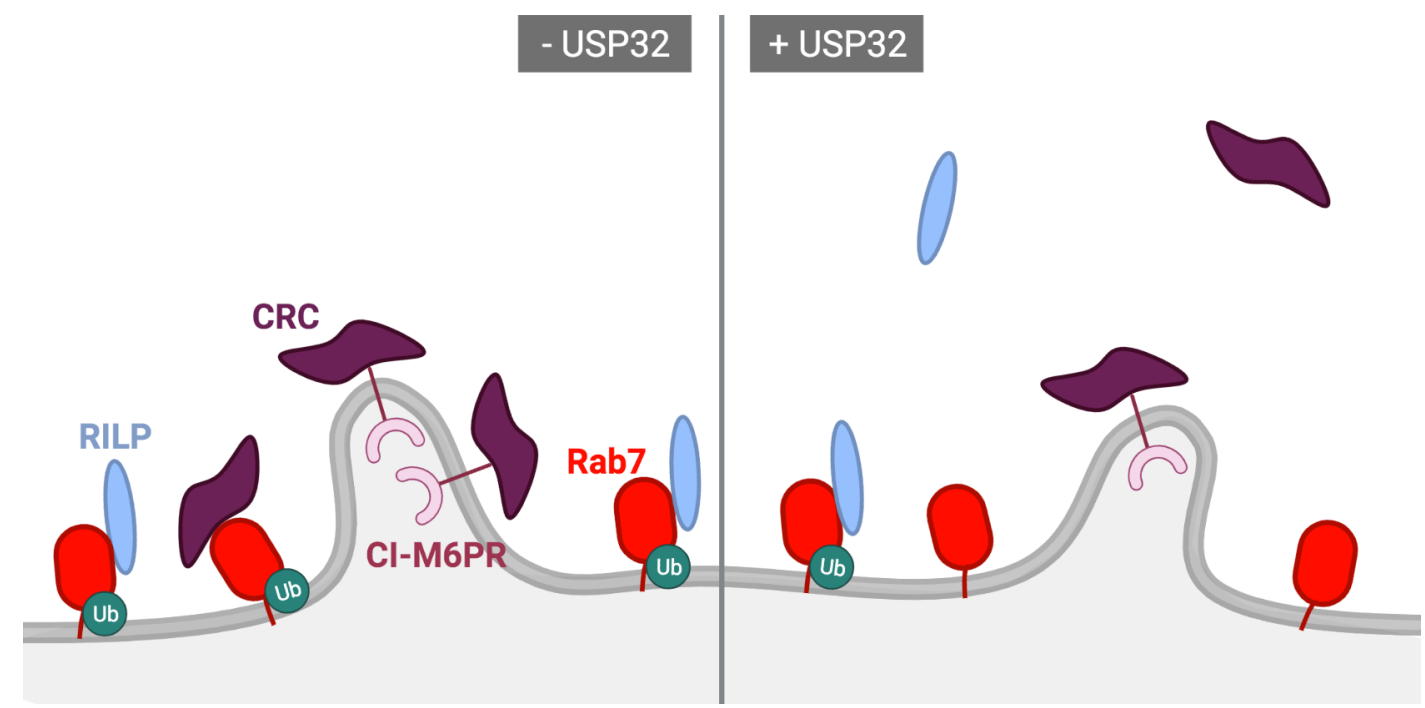

Figure 4-1: Model for the regulation of Rab7 function by USP32 Cartoon showing the hypothesized molecular mechanisms of USP32-sensitive Rab7 ubiquitination at endo-lysosomal membranes. USP32 adjusts the function of Rab7 in vesicle and protein trafficking by deubiquitinating its $\mathrm{C}$-terminus and modulating the interactions of Rab7 and downstream effector protein RILP and retromer CRC.

Besides the interaction and USP32-sensitive ubiquitination of Rab7 at K191/194, the results for Rab7 regulation in absence of USP32 showed that:

(I) Localization of Rab7 at late endosomal structures was not altered.

(II) RILP preferentially bound ubiquitinated Rab7.

(III) Retrograde trafficking of CI-M6PR from the plasma membrane back to the TGN was faster or (IV) more efficient. Overall localization of CI-M6PR at the TGN was enhanced.

(IV) Acidic hydrolases were enriched in lysosomes.

(V) Protein levels as well as composition of the retromer cargo recognition complex (CRC) were stable.

(VI) USP32 co-immunoprecipitated with CRC components.

Based on these findings it was hypothesized that upon depletion of USP32, ubiquitinated Rab7 accumulated. This results in enhanced binding of the effector protein RILP enabling minus-end directed transport of endosomal vesicles on microtubules. Furthermore, recruitment of CRC to its cargo such as Cl-M6PR in sorting tubules is promoted. Thus, CI-M6PR trafficking is more efficient (Figure 4-1 right side "-USP32"). 
This would suggest that USP32 adjusts the function of Rab7 in vesicle and protein trafficking by deubiquitinating its C-terminus. Thereby, the interaction of Rab7 and downstream effector proteins and complexes is modulated (Figure 4-1 left side "+USP32").

\subsection{USP32 regulates non-proteolytic LAMTOR1 ubiquitination and}

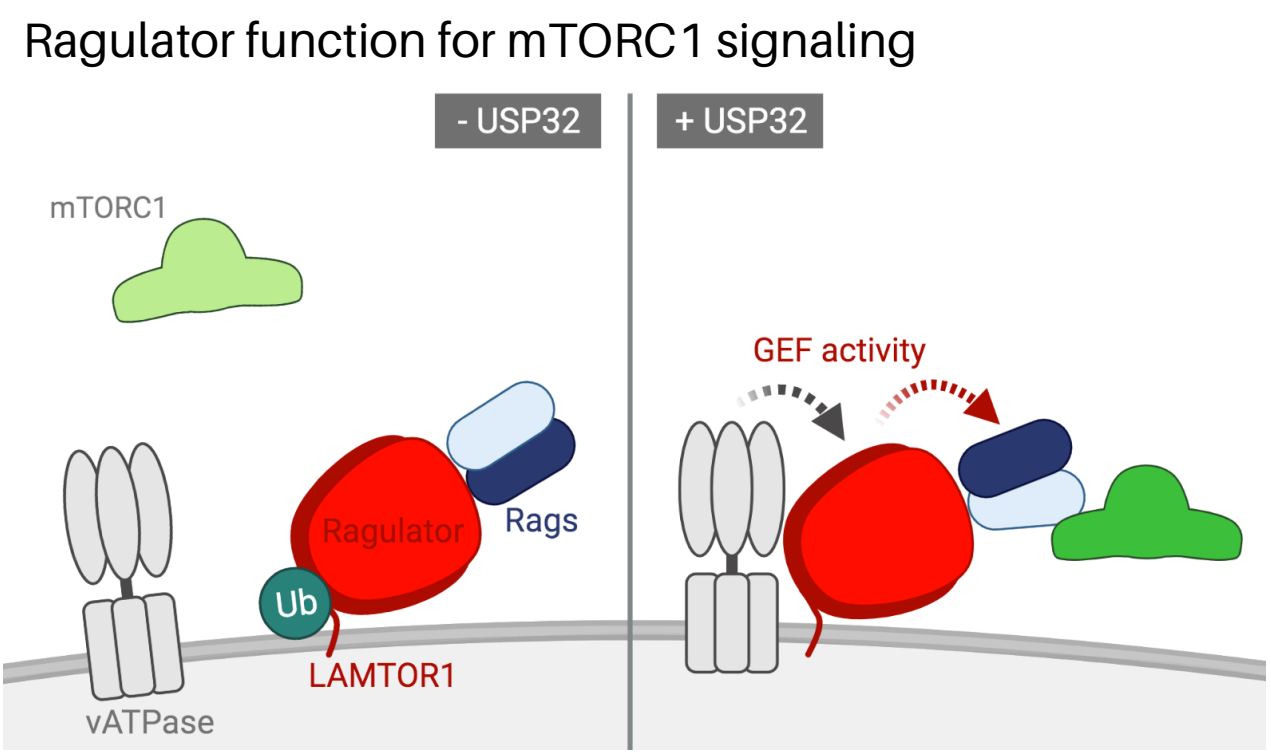

Figure 4-2: Model for the regulation of LAMTOR1 and Ragulator function by USP32 Cartoon showing the hypothesized molecular mechanisms of USP32-sensitive LAMTOR1 ubiquitination at lysosomal membranes. USP32 fine-tunes the interaction of LAMTOR1 and the V-ATPase by deubiquitinating the N-terminus of LAMTOR1. This modulates the recruitment and activation of mTORC1.

Within this work, it could be shown that LAMTOR1 and USP32 co-immunoprecipitated. Furthermore, USP32 significantly deubiquitinated LAMTOR1 at K20, and the USP32-sensitive modification was not dependent on amino acids.

The function of LAMTOR1 ubiquitination upon USP32 depletion was described with following results:

(I) LAMTOR1 localization at lysosomes was decreased independently of amino acid availability. But lysosome positioning was unaffected.

(II) The interaction of LAMTOR1 and subunits of the lysosomal v-ATPase was reduced.

(III) Less mTOR localized to lysosomes independently of amino acid availability.

(IV) mTORC1 activity was lower after starvation and subsequent reactivation.

These results implied that LAMTOR1 is not efficiently deubiquitinated at its $\mathrm{N}$-terminus in the absence of USP32. N-terminal ubiquitination of LAMTOR1 weakens the interaction with the lysosomal v-ATPase. Reduction of this crucial interaction switches mTORC1 in an inactive state in the cytoplasm (Figure 4-2 right side "-USP32").

The presented data suggested that USP32 regulates Ragulator function by fine-tuning the interaction between V-ATPase and LAMTOR1 independently of lysosomal amino acid signals. Unmodified LAMTOR1 interacts more with V-ATPase and stimulates the GEF function of Ragulator. The subsequent full activity of Rag GTPases recruit mTORC1 efficiently to the lysosomal membrane for its activation (Figure 4-2 left side "+USP32").

Both Rab7 and LAMTOR1 regulation eventually converge in the autophagy process by different means. 


\section{Discussion}

\subsection{Establishing tools for the characterization of USP32 function without disease background}

When this project started, Akhavantabasi et al. 2010 presented a basic characterization of the DUB USP32 and a possible involvement of USP32 in breast cancer due to its overexpression. Together with the interactome data of Sowa et al., 2009 and own preliminary data, this study aimed to characterize the cellular function of USP32 without any disease background. Therefore appropriate tools for cell culture based experiments were established. Immortalized hTERT-RPE1 cells were chosen for the majority of experiments since they are non-transformed and diploid in comparison to widely used cancer cell lines. Besides their suitable features for microbiological techniques, transfection of RPE1 cells with lipid-based methods shows low efficiency. In addition, siRNA based gene silencing can have off-target effects. To overcome these two drawbacks, a stable polyclonal cell line with depleted USP32 was generated using the CRISPR/Cas9 technique.

Much effort and time was put in the additional option to overexpress USP32 in cells to make use of the advantage of a tagged or truncated version. This would have overcome the low cellular abundance of USP32 in general and enabled detailed studies of its localization, regulation, and interactions. Despite all made endeavors, the detected exogenous USP32 on an immunoblot ran at lower molecular weight than expected. Though the plasmid DNA sequences were correct, the observations were not clarified so far. It was considered to identify the expressed protein by mass spectrometry. But still the detection could be incomplete if peptides fail to ionize or the ions are unstable and fragmentate (peptides do not "fly" properly).

It was decided to use the CRISPR/Cas9 technique to endogenously tag USP32. In collaboration with Manuel Kaulich (Institute of Biochemistry II, Goethe University Frankfurt), a strategy for a Nterminal RFP- or HA-tagging by homology directed repair was designed, where RPE1 cells would be co-transfected with one plasmid harboring the Cas9 and USP32 gRNA together with a plasmid harboring the USP32 sequence including $5^{\prime}$ and $3^{\prime}$ homology arms of the gene locus. After a long procedure with cloning and transfection issues most probably due to the size of the homology sequence, unfortunately no positive RPE1 cell clone could be obtained. In a possible retry of endogenous USP32 tagging a novel approach with the Cas12 enzyme could be beneficial. Cas12 with its distinct advantages in comparison to Cas9 (e.g. lower mismatch tolerance and greater specificity) was proposed to be more efficient for tagging of mammalian genes (Fueller et al., 2020; Li et al., 2020). 


\subsection{Identification of USP32 substrates in the endosomal-lysosomal system}

Very interestingly, in an unbiased SILAC-based ubiquitinome approach substrates of USP32 in the endosomal and lysosomal system were identified. The resided organelles of these proteins fit perfectly with the cellular localization of USP32 at the perinuclear region at Golgi and lysosomal structures. The detected modified sites of Rab7, Rab11, LAMTOR1 and TMEM192 were reported before (Hornbeck et al., 2015). But the function of the ubiquitin signals was not characterized so far. This study could observe stable protein levels of all four candidates upon USP32 knockout and proteasomal inhibition using LC-MS/MS proteome analysis and immunoblot analysis. This suggested that USP32 did not cleave a proteolytic ubiquitin signal but regulates other signaling functions.

\subsection{Possible ubiquitin regulation of palmitoylation}

Notably, the identified modified residues for Rab7, Rab11 and LAMTOR1 were in the unstructured regions mediating the membrane attachment of the proteins and in close proximity to their lipidation sites. Protein lipidation, in particular prenylation, myristoylation, and palmitoylation, is a post-translational modification (PTM) that regulates many aspects of protein function like localization, stability, trafficking or interactions of several protein families involved in intracellular membrane transport processes. $\mathrm{N}$-terminal myristoylation of a glycine and $\mathrm{C}$ terminal prenylation of a cysteine are irreversible attachments, whereas palmitoylation of a cysteine forms a labile thioester bond and is reversible (Aicart-Ramos et al., 2011; Jiang et al., 2018).

Association of small Rab GTPases with membranes is mediated by the prenylation of the double-cysteine site within their hypervariable C-terminus. With the help of Rab escort proteins (REPs) the Rab geranylgeranyl-transferase (RabGGTase) catalyzes the crucial lipid modification (Andres et al., 1993). Though Wu et al., 2009 present the position of Rab7 K191 in the REPRab7 interaction patch, it appears unlikely that USP32-sensitive Rab7 modification at K191/194 interferes with the prenylation for two reasons: (I) Rabs are modified with a geranylgeranyl moiety briefly after their translation to target them to membranes. USP32 is partially localized at membrane compartments of the endosome-lysosome system concluding that Rab7 and USP32 might meet subsequent to the prenylation process. (II) The C-terminal lipidation of Rab7 is crucial for its membrane attachment. If ubiquitination of Rab7 at lysine 191 or 194 would interfere with its prenylation, a dramatic effect on Rab7 localization would be expected. But in contrast, Rab7 did not change its localization relative to LAMP1 positive structures upon USP32 depletion in RPE1 cells. Noteworthy, Sapmaz et al., 2019 detected increased Rab7 levels in the membrane fraction of cells transfected with siUSP32.

Rab7 palmitoylation at C83/84 independently of its membrane association is required for the interaction with the retromer cargo recognition complex (VPS35, VPS26, VPS19) but not with RILP (Modica et al., 2017). Even though an enhanced retrograde trafficking was observed in USP32 knockout situation, the increased binding of RILP to Rab7 in USP32KO cells and the 
distance between C83/84 and ubiquitinated K191/194 do not directly relate Rab7 palmitoylation and USP32-dependent deubiquitination.

Palmitoyl-proteomic studies supposed that several mTOR pathway components are palmitoylated, including LAMTOR1 (Blanc et al., 2015; Sanders et al., 2015). In addition, LAMTOR1 is co-translationally myristoylated at G2 (presumably by the N-myristoyltransferase-1 (NMT1)), which precedes the dynamic post-translational cysteine palmitoylation (Chen et al., 2020; Farazi et al., 2001). Depletion and inhibition of NMT1 or mutation of G2 abolished colocalization of LAMTOR1 with LAMP1 and induced complete cytoplasmic distribution (Chen et al., 2020; Nada et al., 2009). Results from this study (3.6.2) and from Sun et al., 2018 showed that membrane localization of LAMTOR1 is necessary for its ubiquitination. Thus, it can be excluded that USP32 deubiquitination at K20 of LAMTOR1 regulates myristoylation. But the observed partial loss of LAMTOR1 localization to LAMP2 positive lysosomes is coinciding with the findings of Nada et al. 2009 and Sanders et al. 2019, where the palmitoylation-deficient mutant LAMTOR1 $\mathrm{C} 3 / 4 \mathrm{~S}$ or $\mathrm{C} 3 / 4 \mathrm{~A}$ formed distinct dot structures apart from late endosomes and partially co-localized with the endoplasmic reticulum. Furthermore, mTORC1 signaling in hippocampal neurons was described to be dependent on dynamic LAMTOR1 palmitoylation at C3/4 (Sanders et al., 2019). Comparing the presented data to literature could point to a possible regulation of LAMTOR1 palmitoylation by its USP32-sensitive ubiquitination at K20. In general, regulation of LAMTOR1 palmitoylation processes and the involved enzymes remain elusive till today. Future experiments addressing the palmitoylation status of LAMTOR1 (for example with an acyl biotin exchange assay Thomas et al., 2012) from control and USP32-depleted cells could shed light on this hypothesis and possible novel mechanism.

\subsection{Deubiquitination of Rab7 by USP32}

Rab7 functions are modulated with different post-translational modifications like phosphorylation, lipidation, and also ubiquitination. Global ubiquitinome analysis identified Rab7 lysines at position 38, 191 and 194 as possible ubiquitination sites (Mertins et al., 2013; Wagner et al., 2011). The E3 ligase Parkin was reported to preferentially ubiquitinate Rab7 K38 thereby promoting Rab7 localization at late endosomes and its interaction with RILP (Song et al., 2016). With the detection of the by then uncharacterized USP32-regulated modification of K191 and K194 of Rab7 this project intended to investigate their function in the cellular context. Neither Song et al., 2016 nor this work (or the competing publication by Sapmaz et al., 2019 studied in detail the type of Rab7 ubiquitination or its interaction with the E3 ligase or DUB. Here, interaction of Rab7 and USP32 was shown indirectly by immunoprecipitation from cells with overexpressed tagged proteins (3.5.1). Of course, further biochemical studies could provide insights in the structure and mechanism of ubiquitin chain characteristics, ubiquitination and deubiquitination reactions, and interactions, and thus help understanding the regulation of Rab7 by ubiquitin signals.

Rab7 localization at membranes and binding of effectors is mediated by the C-terminal prenylation and the nucleotide status with various involved proteins. Transition cycles of activation and inactivation by guanine exchange factors (GEFs, only identified GEF for Rab7 
Mon1-Ccz1) and GTPase activating proteins (GAPs like TBC1D2A/B, TBC1D5, TBC1D15), as well as membrane recruitment and extraction by prenylation (RabGGTase and REPs), GDP dissociation inhibitors (GDIs) and GDI dissociation factors (GDFs) tightly control Rab7. The exact mechanism, cellular position and chronological order of all the involved steps is still under investigation and discussion (Kanemitsu-Fujita et al., 2018; Müller and Goody, 2018; Shinde and Maddika, 2016; Stroupe, 2018). As it was shown in the results part (3.5.3), Rab7 did not change its overall localization in USP32 knockout cells, but the interaction with the effector RILP increased. This suggested that the prenylation and membrane recruitment of Rab7 is not impaired by its ubiquitination. Rather the affinity of RILP for Rab7 or the activity of Rab7 regulated by GEFs and GAPs seemed to be controlled by the USP32-sensitive ubiquitin attachment. Structural analysis of the RILP-Rab7 interaction reveals that the ubiquitinated Rab7 residues K38 and K191/194 are either directly in or in close proximity to the interaction areas with RILP (Wu et al., 2005). This data supports the hypothesis that the USP32 deubiquitination of the C-terminus fine-tunes the RILP-Rab7 interaction. It would be interesting for future experiments to test the binding of other effectors like FYCO1.

Up-to-date, there is no study defining the mechanism or structure of the GTP-GDP-switch of Rab7 mediated by GEFs and GAPs. Together with the presented data, the question whether Rab7 activity and the associated nucleotide status is changed by K191/194 modification can't be further discussed and detailed studies would be necessary. However, measuring the bound nucleotide directly is only possible in radioactively labeled in vitro assays or indirectly by expressing constitutively active or inactive Rab7 mutants (GTP bound Q76L, GDP bound T22) for comparison (Gutierrez et al., 2004; McCray et al., 2010). Conclusively, determination of ubiquitinated Rab7 nucleotide status from USP32KO cells could clarify if enhanced Rab7GTP activity or enhanced affinity causes RILP binding.

\subsubsection{Effect of Rab7 ubiquitination on trafficking}

Rab7 function and also K38 ubiquitination was reported to be involved in the retromer mediated recycling from late endosomes (Rojas et al., 2008; Seaman et al., 2009; Song et al., 2016). CI-M6PR, the sorting receptor for lysosomal hydrolases, is a well-known substrate of retromer and its transport was used as model route in this work to investigate the effect of altered Rab7 activity upon USP32-sensitive ubiquitination. Transport of CI-M6PR seemed to be more efficient or faster in USP32-depleted cells resulting in increased cathepsin levels in lysosomes. The described phenotypes follow the results on Rab7 activity/RILP binding. Rab7 has several key roles in trafficking and USP32 deubiquitination could interplay at various stages (see 1.1.5). Coordination of RILP binding could positively control vesicle fusion as well as minus-end directed transport on microtubules. Furthermore, recruitment of the retromer CRC to membranes mediated by Rab7 could benefit from ubiquitination and USP32 could adjust the initiation of recycling. Notably in this context, detected interaction of USP32 and retromer CRC in 3.5.7.2 confirmed the interactome of Sowa et al., 2009. USP32 depletion showed also no impairment or CRC component or complex stability, which goes along with the definition of CRC as a stable trimer (Seaman, 2012). A closer examination of the Rab7 interaction landscape would contribute to the further understanding of USP32 modified Rab7 function. 
In addition to an USP32-dependent Rab7 interactome, detailed investigation of other Rab7 mediated pathways or endosomal morphologies would give interesting insights in regulation by dynamic ubiquitination of Rab7 K191/194. Similar experiments were conducted for other post-translational modifications. K38 ubiquitination by Parkin displayed involvement in endosomal membrane tabulation since a decrease in endosomal tabulation was observed in Parkin-deficient fibroblasts by live cell microscopy (Song et al., 2016). Phosphorylation of Rab7 S72 and Y183 was identified as regulatory PTM for EGFR trafficking and subsequent degradation (Francavilla et al., 2016; Lin et al., 2017; Shinde and Maddika, 2016).

The characterization of Rab7 as a USP32 substrate may also be relevant from a medical perspective. Rab7 and its downstream effector retromer CRC have been implicated in neurological (Charcot-Marie-Tooth disease, Spinosa et al., 2008) and neurodegenerative diseases (Alzheimer's and Parkinson's, Li et al., 2016; McMillan et al., 2017). Alterations of Rab7 and retromer functions due to up- or downregulated protein expression or mutations impaired endosomal membrane dynamics and transport as well as lysosome degradation capacity (Follett et al., 2014; Ginsberg et al., 2011; Li et al., 2016; Williams et al., 2017). Targeting USP32 for modulating the function of Rab7 and subsequently retromer by reversible ubiquitination could offer a novel therapeutic approach.

\subsubsection{Competing data on Rab7 regulation through USP32 deubiquitination}

This thesis studied the cellular function of the back then uncharacterized deubiquitinating enzyme USP32 and identified Rab7 as novel substrate. Though Rab7 ubiquitination by Parkin on K38 and its function was already reported, modification of K191/194 was only reported but not studied in detail (Mertins et al., 2013; Song et al., 2016; Wagner et al., 2011). Furthermore, no DUB was identified as Rab7 deubiquitinating enzyme. Majority of the data on Rab7 regulation was generated during the first part of this PhD project implying that USP32 deubiquitination of K191/194 restricts RILP binding and possibly Rab7 activity in trafficking.

At the beginning of 2019 Sapmaz et al., 2019 published their findings on Rab7 regulation by USP32. The competing work described congruent but also contradictory results to the here presented results. They identified USP32 as an endosomal regulator in a siRNA based DUB screen in human melanoma MelJuSo cells showing altered redistribution of MHC-II to the cell periphery and surface in absence of USP32. The initial findings were verified in HeLa cells using EGF staining upon siRNA transfection. After describing USP32 as a membrane-associated catalytically active DUB, Rab7 was established as USP32 substrate in a SILAC based LC-MS/MS ubiquitinome again with siRNA or overexpressed USP32. In the further experiments, they showed dramatic relocalization of Rab7 to membranes in the periphery upon USP32 knockdown, increased binding of RILP to an ubiquitination-deficient Rab7 mutant, but decreased binding of ubiquitinated Rab7 and VPS35. This paper suggested that USP32 deubiquitination of Rab7 controls membrane dynamics at late endosomes by promoting their intracellular motility as well as enabling efficient Rab7 recycling from these organelles.

Compared to the results of this work, Sapmaz et al., 2019 showed similar data on the characterization of USP32, the identification of Rab7 as a USP32 substrate and the modified residues K191 and K194. Enhanced binding of VPS35 to ubiquitinated Rab7 would also be 
consistent with the here observed faster retrograde transport of Cl-M6PR meaning efficient recruitment and binding of retromer to Rab7 positive recycling structures would enable efficient and fast transport of CI-M6PR back to the TGN.

However, contradictory data was worked out in this study, as well. Rab7 localization was not altered. The distribution of late endosomal cargo containing vesicles (here CI-M6PR, Sapmaz et al., 2019 EGFR) was shifted to the perinuclear region and not to the cell periphery. Additionally in contrast to Sapmaz et al., 2019, RILP precipitated more ubiquitinated Rab7 upon loss of USP32.

These differences could arise from the performance of the experiments in different cell types. Sapmaz et al., 2019 used two cancer cell lines, melanoma MelJuSo and cervical adenocarcinoma HeLa cells. Retinal RPE1 and osteosarcoma U2OS cells were used in this study. Alterations in cancer cell lines could always lead to different outcomes when compared to cells from normal tissue. Nevertheless, most of the experiments in RPE1 cells were successfully repeated in U2OS cells with similar results. Secondly, this study was conducted with a pooled CRISPR/Cas9 knockout cell line on purpose to work with a loss of USP32 on DNA level instead of siRNA mediated depletion on mRNA level. A pooled cell line was chosen to diminish possible single clone off-target effects. Sapmaz et al., 2019 used an siRNA pool for their initial screen but only one single siRNA was used for all following experiments. Of course, one has to keep in mind that both depletion methods siRNA and CRISPSR/Cas9 have advantages and disadvantages over the other.

\subsection{Deubiquitination of LAMTOR1 by USP32}

Since its first report in 2009 (Nada et al., 2009) many studies described LAMTOR1 and Ragulator complex function in amino acid-dependent mTORC1 activation. LAMTOR1 tethers the Ragulator complex to the lysosomal membrane, which is essential for its function (Nada et al., 2009; Sancak et al., 2010; Sun et al., 2018). Most recent publications suggested that Ragulator is a guanine exchange factor for the RagA/B-RagC/D dimer, which recruits mTORC1 to the lysosomal surface. The GEF activity would be stimulated via amino acid sensing of the lysosomal V-ATPase (Bar-Peled et al., 2012). Another amino acid sensor SLC38A9 was discovered to control mTORC1 by its GEF activity toward the Rags (Jung et al., 2015; Rebsamen et al., 2015; Shen and Sabatini, 2018). Structural and mechanistical observations implied that both Ragulator and SLC38A9 are each unique GEFs that collectively push the Rag GTPases toward the active state where Ragulator triggers GTP release from RagC and SLC38A9 converts RagA to its GTP- bound state (Shen and Sabatini, 2018). Furthermore, SLC38A9 was shown to activate GAP function of Folliculin:FNIP2 toward RagC thereby promoting Rag dimer activation upon amino acid stimulation (Fromm et al., 2020).

Besides Ragulator regulation by v-ATPase amino acid sensing, also two PTMs on LAMTOR1 were characterized to be involved in Ragulator and mTORC function. Myristoylation and dynamic palmitoylation of LAMTOR1 N-terminus was necessary for mTORC1 signaling (Chen et al., 2020; Sanders et al., 2019). The E3 ligase Ube3a mediated LAMTOR1 ubiquitination 
preferentially on $\mathrm{K} 60$ and $\mathrm{K} 103 / 104$ in mice leading to its proteasomal degradation and reduction of $\mathrm{mTORC} 1$ activity (Sun et al., 2018).

This PhD thesis identified LAMTOR1 as USP32 substrate and reported the first deubiquitinating enzyme to modify LAMTOR1. USP32 cleaved non-proteolytic ubiquitin signals from LAMTOR1 K20 significantly but also other residues were detected to be modified. A myristoylation-deficient LAMTOR1 G2A mutant revealed that lysosomal localization of LAMTOR1 was required for its ubiquitination, suggesting that USP32 specifically targeted LAMTOR1 at lysosomes. Additionally, the ubiquitination status of an ubiquitination-deficient LAMTOR1 K20R mutant suggested that LAMTOR1 is also efficiently ubiquitinated at additional lysine residues. Congruent with Sun et al., 2018, K60 showed the highest modification intensity. This implied that USP32-sensitive LAMTOR1 ubiquitination at K2O rather adjusts than controls a signal. Similar to the data for Rab7, the detailed biochemical and structural analysis of LAMTOR1 ubiquitination was not attempted neither by this study nor by Sun et al., 2018. The interaction of LAMTOR1 and USP32 was again shown indirectly by immunoprecipitation from cells with overexpressed tagged proteins. Future identification of the structure and mechanism of ubiquitin chain characteristics, ubiquitination and deubiquitination reactions, and direct interactions, could contribute to the understanding of LAMTOR1 ubiquitin signals.

\subsubsection{Effect of USP32-sensitive ubiquitination on LAMTOR1}

Ubiquitinated LAMTOR1 localization at LAMP2-positive lysosomes was decreased upon USP32 depletion independently of amino acid availability. Sun et al., 2018 observed a similar phenotype with increased LAMP2-LAMTOR1 co-localization in neurons from Ube3a-deficient mice with Angelman syndrome (AS). However, Ube3a mediated the proteasomal degradation of LAMTOR1. Thus, stabilization of LAMTOR1 in AS mice could explain its elevated levels at lysosomes. In contrast, USP32 did not regulate a proteolytic ubiquitin signal on LAMTOR1. LAMTOR1 localization at lysosomal membranes is achieved by its $\mathrm{N}$-terminal lipidation, namely glycine myristoylation at position 2 and cysteine palmitoylation at position 3 and 4 (Nada et al., 2009; Sanders et al., 2019). The here presented result for LAMTOR1 localization hinted that the USP32-sensitive ubiquitination at K20 could interfere with the lipidation of LAMTOR1. As discussed before (5.3), the obtained data rather suggested a possible control of palmitoylation than myristoylation.

Ragulator is important for the positioning of lysosomes within the cell via its interaction with BORC. Under basal, amino acid rich conditions, Ragulator frees BORC on lysosomes to promote the coupling to kinesins for plus end directed movement. Under amino acid-depleted conditions, Ragulator inhibits BORC. Lysosomes become uncoupled from kinesins and cluster at the perinuclear region (Filipek et al., 2017; Pu et al., 2017). To test whether USP32 and LAMTOR1 ubiquitination could influence lysosomal positioning, the radial distribution of LAMP2 dots in untreated and starved cells lacking USP32 was measured. USP32 knockout did not induce significant changes in lysosome distribution. Notably, the quantification did not indicate a complete clustering in the perinuclear region upon starvation, as the LAMP2 intensity in this region did not increase significantly. Further experiments to clarify if USP32 affects lysosomal positioning would need an optimized setup considering following aspects: 
quantification of the distance to the nucleus or microtubule-organizing center (Pu et al., 2017) instead of radial distribution to avoid effects of polarization toward one side of the cell, different cell type since RPE1 cells have little cell bodies and might not be suitable for proper dissection, other time-points or live cell imaging for dynamic processes (compare (Filipek et al., 2017; Pu et al., 2017).

\subsubsection{USP32-dependent LAMTOR1 interactome studies}

In comparison to the ubiquitinome analysis, the interactome was performed in a label-free manner. The low sample complexity enabled a fast and uncomplicated preparation with comparable LAMTOR1-GFP enrichment from NHT and USP32KO cells. The identification of two subunits of the lysosomal v-ATPase with decreased interaction to LAMTOR1 in absence of USP32 linked the function of K20 ubiquitination and deubiquitination to Ragulator's role in mTORC1 signaling. Besides its action as proton pump for lysosomal acidification, the v-ATPase was reported to interact with Ragulator and to link the amino acid-generated signal to the activation of Ragulators GEF activity (Bar-Peled et al., 2012). A few studies proposed models how and under which conditions Ragulator and V-ATPase interact stronger or weaker, respectively (Chung et al., 2019; Zoncu et al., 2011). These findings will be discussed later in the context of mTORC 1 activation.

The first interactome under basal conditions revealed 28 proteins with significantly changed interaction to LAMTOR1 in absence of USP32. The second interactome including starvation and reactivation detected only four proteins in untreated samples as significantly changed. Similar numbers $(\leq 10)$ for significantly up- or downregulated interactions were resolved when comparing between same treatment, or same cell line. This implied that the quality of the experiment was lower than for the first experiment. Though mass spectrometry quality measures (digestion efficiency, retention time reproducibility, normalized protein intensities) were evaluated as good in both experiments, the number of identified proteins was $34 \%$ less in the second experiment (experiment 1 2250, experiment 2 1476). Also the number of significantly enriched proteins from LAMTOR1-GFP IPs in comparison to the GFP was reduced. This implied that issues might have arisen from the sample preparation rather than from the LCMS/MS analysis. Here, sample labeling would facilitate the handling of complex experiment such as for the second interactome with treatments.

As described in the introduction (1.2.1) and results part (3.6.5), a number of protein complexes assemble at the lysosomal membrane to mediate amino acid signals to mTORC1. The interactome studies intended to identify interactions of LAMTOR1 with these proteins. However, only the four other LAMTOR (2-5) proteins and RHEB were detected besides the vATPase subunits. No other peptides of known interactors appeared. All interactions of the mTORC1 activation process for subsequent recruitment, activation, and release are tightly regulated and have to be very transient and short-termed (Lawrence et al., 2018). Thus, they are not trivial to investigate. Earlier studies applied optimized protocols including special buffers for immunoprecipitations, tandem-affinity-purifications, cross-linking or proximity-labeling before precipitation (Jia et al., 2018; Jung et al., 2015; Rebsamen et al., 2015; Sancak et al., 2008; Zoncu et al., 2011). The immunoprecipitations within this work were executed under standard 
lysis conditions without prior cross-linking or labeling. Few changes in the IP protocol were already adapted to verify the USP32-dependent interaction of LAMTOR1 and V-ATPase subunits, but so far with unsuccessful outcome.

\subsubsection{Effect of USP32-sensitive ubiquitination of LAMTOR1 on mTORC1}

The observed decreased interaction of ubiquitinated LAMTOR1 with V-ATPase subunits suggested a subsequent impairment of the Ragulator-Rags-mTORC1 relation. Indeed, upon depletion of USP32 the localization of mTOR relative to LAMP2 decreased independently of amino acid availability, and activity of mTORC1 was diminished. It was hypothesized that a consequence of weakened LAMTOR1-V-ATPase interaction (by USP32-sensitive ubiquitination of K20) was a lower Ragulator GEF activity for the Rag GTPases. Hence, Rags would remain in their inactive RagA/B ${ }^{G D P}-$ RagC/D ${ }^{\text {GTP }}$ status in absence of USP32. mTOR would not be recruited to the lysosomal surface and would stay inactive in the cytoplasm. Conclusively, USP32 would adjust mTORC1 activation independently of amino acid availability by enabling the interaction of v-ATPase and Ragulator, and subsequent activation of Rags.

This project could produce data for the interaction of LAMTOR1 and V-ATPase as well as for the mTOR localization and activation. Nevertheless, data on Rag GTPases are missing.

Literature proposed following models for the interactions at the lysosomal membrane for starved and fed conditions as well as with two v-ATPase inhibitors SalA and EN6 (Bar-Peled et al., 2012; Chung et al., 2019; Zoncu et al., 2011):

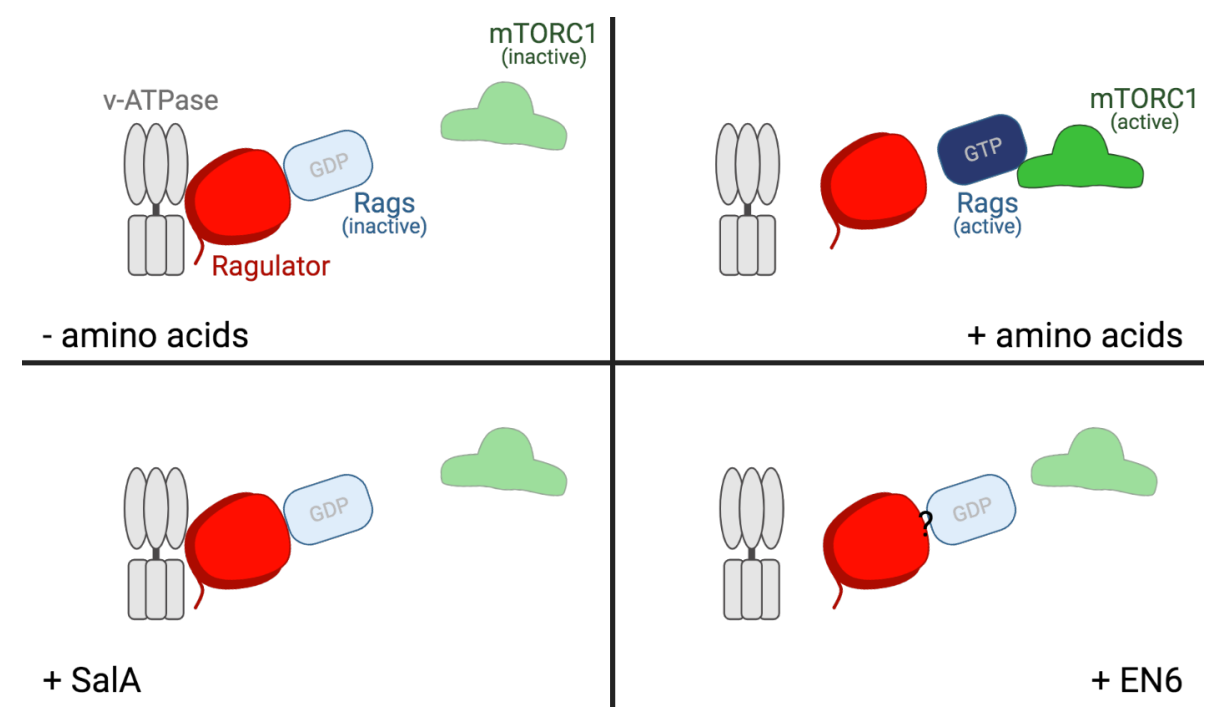

Figure 5-1: Models of V-ATPase-Ragulator-Rag GTPases-mTORC1 interactions In amino acid deprived conditions Ragulator showed enhanced interaction with v-ATPase and inactive GDP-bound Rags, mTORC1-Rag interaction was low. In amino acid rich conditions Ragulator showed reduced interaction with v-ATPase and active GTPbound Rags, mTORC1-Rag interaction was enhanced. Addition of V-ATPase inhibitor SalA mimicked starved conditions. In presence of v-ATPase inhibitor EN6 the interaction of Ragulator and v-ATPase was decreased, mTORC1 was not recruited to lysosomes.

According to Chung et al., 2019 showing a reduced v-ATPase interaction of LAMTOR1 and decreased mTORC1 activity in presence of EN6, the data of this PhD thesis would hint to inactive GDP-bound Rag GTPases and therefore higher affinity of Ragulator toward the Rags. 
Notably, enriched RagC/D levels in purified lysosomes from USP32KO cells were detected in the SILAC proteome, what would fit the hypothesized theory of more inactive Rag GTPases.

To fully understand the function of LAMTOR1 deubiquitination by USP32 and the regulatory mechanism resulting in adjusted mTORC1 signaling, future studies have to focus on this missing link between LAMTOR1/Ragulator and mTORC1. It would be of high interest to examine the LAMTOR1/Ragulator binding to Rags and the nucleotide status of Rags. Both aspects would require advanced experimental techniques and workarounds due to their transient character. As already discussed in the context of v-ATPase, detecting the interaction of LAMTOR1 and Rag GTPases might benefit from specialized protocols like proximity labeling. Tests with modified buffer conditions for immunoprecipitations of either endogenous or overexpressed proteins were not robust until now. The direct measurement of the bound nucleotide to RagA/B or RagC/D would not be possible form cells. Previous publications used controlled GTP/GDP loading with radioactively labeled xanthosine in vitro or expression of constitutively active or inactive Rag mutants (e.g. inactive RagB T54N, active RagB Q99L) (BarPeled et al., 2012; Lawrence et al., 2018).

The key regulator of anabolic processes in cells mTORC 1 coordinates the balance between cell growth and cell death and is frequently activated in cancer. Mutations in mTORC1 itself or components of its signaling pathways have been implicated in cancer, as well. Therefor it is no surprise that mTORC1 and its (de-)regulation are well studied targets for cancer treatments (Kim 2019). Even more interesting from a medical point of view in the context of USP32 are the recent publications describing the role of LAMTOR1-dependent $\mathrm{mTORC} 1$ regulation in neurons and neuronal disease. In a system biology approach to discover novel biomarkers for Alzheimer's disease (AD), LAMTOR1 was identified as a novel biomarker and proposed to be related to AD (Soleimani Zakeri et al., 2020). Furthermore, LAMTOR1 was found to be overexpressed on gene and protein levels in subependymal giant cell astrocytomas (SEGAs), which are characteristic for tuberous sclerosis complex in the central nervous system (Bongaarts et al., 2020). Most related to the findings of this thesis is the ubiquitin-dependent regulation of LAMTOR1 by Ube3a in the Angelman syndrome (AS). Sun et al., 2018 presented mTORC1 signaling downstream of LAMTOR1 in AS mice as critical for typical synaptic plasticity, dendritic spine development, and learning and memory.

The here described adjustment of LAMTOR1 (and Ragulator) function in mTORC1 signaling by USP32 could lead to new approaches in the medical research on neurological disorders.

\subsection{Autophagy}

Autophagy, the process of self-degradation, plays an important physiological role in response to various cellular stresses and enables the cell to survive such starvation conditions. Autophagy and the endocytosis do not just both deliver cargo to lysosomal degradation and provide nutrients and macromolecules for the cell. Both pathways are in close cooperation as they overlap at different stages. Membranes of endocytic vesicles and recycling endosomes can contribute to phagophore formation (Longatti et al., 2012; Ravikumar et al., 2010). Furthermore, common molecular motors transport endosomes and autophagosomes along 
microtubules (Maday et al., 2012). The lysosomal compartment is the central hub for sensing the cellular nutrient status and controls the switch between anabolism and catabolism. So autophagy is initiated and terminated at lysosomes.

The here characterized substrates of USP32, Rab7 and LAMTOR1, are already described to play a role in autophagy. Rab7 is important in docking, fusion, transport, and clustering of autophagosomes during the entire autophagy process (Gutierrez et al., 2004; Jäger et al., 2004). LAMTOR 1 and the Ragulator complex are required to transfer lysosomal nutrient signals to $\mathrm{mTORC} 1$ as the master regulator of autophagy. TFEB is a direct mTORC1 substrate and functions on transcription of autophagy genes in response to starvation (Settembre et al., 2011). Additional substrates of mTORC1 determine autophagy induction, nucleation, elongation, and maturation and fusion (Dossou and Basu, 2019). Besides the USP32 substrates themselves, also related proteins and complexes were reported to be involved in autophagic processes. Retromer, lysosomal V-ATPase, and hydrolases contribute eventually to proper function and high degradation capacity of lysosomes unrelated to autophagy genes (Inami et al., 2011; Yim and Mizushima, 2020). Taken together, Rab7- and mTORC1-dependent pathways converge in autophagy. Therefor it was just consequent in this thesis to proof that USP32 impaired autophagy by regulating the function of Rab7 and LAMTOR1. Though Sapmaz et al., 2019 partially described the cellular role of USP32 in the context of Rab7, its involvement in autophagy was presented in this study for the first time. Enhanced LC3-positive autophagosomes in USP32KO cells suggested USP32 as negative regulator of autophagy. Nevertheless, a more diverse function at different stages of the endosomal and lysosomal system with an overall influence on early and late autophagy steps could not be excluded. A FACS based experimental approach using a tandem fluorescent GFP-LC3-RFP could be used to dissect the mechanism in more detail. This probe enables the quantitative evaluation of the autophagic flux in a pool of cells (Kaizuka et al., 2016).

Furthermore, it would be interesting to examine the effect of USP32 on TFEB. An inhibition of mTORC1 would imply the nuclear translocation of TFEB. Preliminary microscopy experiments in USP32-depleted cells used either exogenous or stably expressed GFP-TFEB as well as staining of the endogenous TFEB. However, the results were inconsistent so far and further optimization of the experimental set-up is necessary. Notably, lysosomes isolated from RPE1 USP32KO cells showed enriched cathepsin Z levels both by Western blotting (3.5.6.2) as well as in the proteome analysis of the secretome (3.5.6.4). This would hint that the expression of cathepsin Z could be induced by TFEB, which would support the hypothesized decreased mTORC1 activity.

\subsection{Rab11 and TMEM192 as USP32 substrates}

Besides Rab7 and LAMTOR1 two other proteins of the endosomal and lysosomal system were identified as possible USP32 substrates: Rab11 and TMEM192.

Rab11 is the key regulator of recycling endosomes (RE), which mediate the slow transport back to the plasma membrane in a tubovesicular compartment (Jovic et al., 2010). Like other members of the Rab GTPase family, Rab11 is prenylated at its C-terminus enabling its 
membrane attachment (Gromov and Celis, 1998). Its functions were reported to be important for recycling endosomes in PtdIns(3)P turnover at early endosomes, subsequent tabulation and release of recycling cargo from early endosomes, transport of REs along microtubules to the plasma membrane, and eventually exocytosis of cargo (Campa et al., 2018; Takahashi et al., 2012; Wilcke et al., 2000). Furthermore, Rab11 mediated processes are involved in various disease such as different types of cancer, neurodegenerative disease like Alzheimer's and Huntington's, as well as infections by pathogens (Bhuin and Roy, 2015; Guichard et al., 2014).

It seems likely that regulation of Rab11 by USP32-sensitive ubiquitination might also contribute to the described observations in trafficking and autophagy. The characterization of Rab11 as a USP32 substrate in the course of a new project would further shed light on the regulation of Rab GTPases and trafficking by ubiquitin signals.

TMEM192 was discovered in 2010 as novel lysosomal transmembrane protein but its physiological role is still unknown. Its depletion in normal mouse tissue had no observable effect whereas in HepG2 hepatoma cells apoptosis and autophagy was induced (Liu et al., 2012; Nguyen et al., 2017). Recent studies identified TMEM192 to be ubiquitinated upon lysosomal damage (Yoshida et al., 2017). Interestingly, peptide-tagged TMEM192 is used as a tool to purify lysosomes from cells (Singh et al., 2020). The characterization of the still novel TMEM192 is just starting. Investigating its ubiquitination and deubiquitination by USP32, maybe in the context of lysosomal damage, would generate data on how PTMs regulate TMEM192.

\subsection{Regulation of USP32}

This work and others could shed light on the cellular function of USP32. In addition, it was shown to be an active DUB with low linkage type specificity (Akhavantabasi et al., 2010; Sapmaz et al., 2019). Nevertheless, it is still unknown how USP32 itself is regulated. The domain structure of USP32 contains two very interesting domains that are unique in the entire DUB family. The N-terminal EF hands are promising for a possible calcium regulation. Endosomes and lysosomes have a central role in intracellular $\mathrm{Ca}^{2+}$ signaling since they are important cellular $\mathrm{Ca}^{2+}$ storage compartments. Lysosomal calcium signaling regulates the phosphatase calcineurin and TFEB, and thus autophagy (Medina et al., 2015). Showing the link of US32 localization at endosomal-lysosomal compartments and calcium regulation via its EF hands could add a new layer of DUB regulation in general and characterization of USP32.

Even more intriguingly in the context of endosomal-lysosomal system and the identified substrates is the C-terminal prenylation site of USP32. As described already, Rab GTPases as well as numerous proteins in the mTORC1 signaling pathway are decorated with prenyl, myristoyl, and palmitoyl groups. In contrast to USP32, Rab GTPases lack the CAAX motif but exhibit a variety of prenylation motifs at their C-termini. Substrate recognition by the Rab geranylgeranyl transferase requires binding to Rab escort proteins before Rabs are modified by geranylgeranyl groups (Anant et al., 1998).

Besides the Rab geranylgeranyl transferase, two other prenyl transferases are known. The protein farnesyl transferase (PFT) and the protein geranylgeranyl transferase-type I (PGGTI) 
define the functional class of CAAX prenyl transferases by recognizing a CAAX ( $C=$ cysteine, $A=$ aliphatic residue, $\mathrm{X}=$ any amino acid) tetrapeptide prenylation motif at the $\mathrm{C}$-terminus of their substrates. The residue at position $X$ is sufficient to determine which enzyme will modify which protein. When $X$ is a methionine or serine the protein is farnesylated by PFT. When $X$ is a leucine, then the protein is geranylgeranylated by PGGTI (Casey and Seabra, 1996; Pereira-Leal et al., 2001). However, USP32 contains a glutamine as last amino acid. Thus, a predication which prenyl transferase might modify USP32 is not possible. Due to its reported localization at membranes, it is assumed that USP32 is indeed prenylated. Novel investigations to confirm USP32 prenylation in general and which prenyl transferase mediates the modification are needed. This would open a complete new field since no other DUB was described to be prenylated so far.

This project could identify substrates of USP32 in the endosomal and lysosomal system. Further analysis of the USP32-sensitive ubiquitination on Rab7 and LAMTOR1 presented a role for USP32 in protein and membrane trafficking as well as in mTORC1 signaling. Both pathways converge by affecting autophagy as secondary effect. Though the cellular function of USP32 could partially be unrevealed, many questions remain open. Further investigation will shed light on Rab7 and LAMTOR1 regulation by USP32 deubiquitination in more detail. New insights not just in its substrates but also in the regulation and possible modification of USP32 itself will be very interesting in the field of DUBs and intracellular trafficking. Future results might then serve for new therapeutical approaches in various diseases such as different types of cancer as well as neurodegenerative diseases. This thesis is a starting point for many more projects. 


\section{$6 \quad$ Materials and methods}

\subsection{Materials}

\subsubsection{Devices}

Name

Aqualine AL5 water bath

Avanti J-26 XP + Rotor JLA 10.500

BioSpectrometer basic + $\mu$ cuvette G1.0

Centrifuge 5425R

ChemiDoc MP imaging system

Concentrator plus speed-vac

EASY-nL 1200 System

Ecotron incubator/shaker

Heracell 150i CO2 incubator

Herasafe laminar flow hood

Heratherm copact bacterial incubator

LSM 780 confocal laser scanning microscope

MacBook Pro

Magnetic rack 16-Tube SureBead

Magnetic stirrer+heating

Mastercycler Nexus X2

Megafuge 16R + Rotor TX-400

MicroStar $17(\mathrm{R})$

Microwave

Mini Trans-Blot Cells

Mini-Protean Tetra Cells

Company

Mini-Sub Cell GT Cell

Lauda

Beckman Coulter

Eppendorf

Eppendorf

Bio-Rad

Eppendorf

Thermo Scientific

Infors HT

Thermo Scientific

Thermo Scientific

Thermo Scientific

Zeiss

Apple

Bio-Rad

VWR

Eppendorf

VWR

VWR

Siemens

Bio-Rad

Bio-Rad

Bio-Rad

nanoFlex ion source

Thermo Scientific

Optima XE

Beckman Coulter

$\mathrm{pH}$ meter

PowerPac Basic Power Supplies

Mettler-Toledo

PowerPac HC High-Current Power Supply

Bio-Rad

Bio-Rad

$\mathrm{Q}$ exacive $\mathrm{HF}$

Scale M-Pact AX623

Thermo Scientific

Sartorius

See-saw rocker SSL4

Stuart equipment

Sonicator ultrasonic processor

Qsonica

Sprout mini centrifuge

Synergy $\mathrm{H} 1$ microplate reader

Heathrow Scientific

TCS SP8 confocal microscope

BioTek

Thermomix F1.5

Leica

Tube roller

Vac-Man ${ }^{\circledR}$ Laboratory Vacuum Manifold

Eppendorf

Vacusip aspirator

Vacuum pump VP820

Vortex Genie 2

Stuart equipment

Promega

Integra Biosciences

VWR

Scientific Industries 


\subsubsection{Software and online tools}

\begin{tabular}{ll} 
Name & Company \\
\hline Adobe Illustrator CS5 & Adobe Systems \\
CellProfiler 3.1.9 & \\
Image Lab 5.2.1 & Broad Institute (Carpenter et al., 2006) \\
ImageJ 1.52q & Bio-Rad \\
In-Fusion molar ratio calculator & National Institute Health (Schneider et al., 2012) \\
LAS X & Takara Bio \\
MaxQuant 1.5.1, 1.6.14 & Leica \\
Office for Mac 2011 & Max-Planck-Institute of Biochemistry (Cox and Mann, \\
Papers ${ }^{3}$ for Mac 3.4.25 & 2008) \\
Perseus 1.6.7 & Microsoft \\
Prism 5.0b & Digital Science \& Research Solutions \\
QuikChange Primer Design & Max-Planck-Institute of Biochemistry (Tyanova et al., \\
Program & 2016b) \\
Snapgene 2.8.3 & GraphPad Software \\
Zen & Agilent \\
& GSL Biotech
\end{tabular}

\subsubsection{Chemicals, reagents, labware}

\begin{tabular}{lll} 
& & Catalogue \\
Name & Company & number \\
\hline 2-Propanol AnalaR & VWR Chemicals & 20842 \\
Acetic acid & Thermo Fisher Scientific & BP1185 \\
Acetone & Carl Roth & 7328 \\
Acetonetrile & Carl Roth & AE70 \\
Acrylamide solution (40 \%) & Applichem & A4989 \\
Albumin Fraction V, protease-free (BSA) & Carl Roth & T844 \\
Amersham ECL Prime Western Blotting & GE Healthcare Life Sciences & RPN2232 \\
Detection Reagent & & \\
Amicon Ultra-0.5 mL centrifugal filters (3 kDa) & Merck & UFC5003 \\
Ammonia solution 25\% & Merck & 105432 \\
Ammonium bicarbonate (ABC) & Sigma-Aldrich & 285099 \\
Ammonium peroxydisulphate (APS) & Carl Roth & 9592 \\
Aprotinin & Applichem & A2132 \\
Benzonase (25,000U) & Merck & $71205-3$ \\
BradfordUltra & Expedeon & BFU1L \\
Bromphenolblue & Applichem & A2331 \\
Chloroacetamide (CAA) & Sigma-Aldrich & 22790 \\
cOmplete EDTA-free protease inhibitor tablets & Roche & 4693132001 \\
Control magnetic beads & LifeSensors & UM400M \\
Dithiotreitol (DTT) & Carl Roth & 6908 \\
DQ Red BSA & Thermo Fisher / Invitrogen & D12051 \\
Econo-Column Chromatography Column & Bio-Rad & 7372522 \\
EDTA for molecular biology & Applichem & A5097 \\
Ethanol gradient grade for liquid & Merck Millipore & 111727 \\
& & \\
& &
\end{tabular}


chromatography LiChrosolv

Formic acid

Merck

111670

GFP-Trap beads

Glutathion red. F. Bioch.

Chromotek

gta

Sigma-Aldrich

G4251

Glutathion-Agarose 4B

Machery-Nagel

745500

Glycerol

Glycine for molecular biology

Applichem

A2926

Applichem

A1067

Guanidine hydrochloride

Carl Roth

6069

Hydrochloric acid $(\mathrm{HCl})$ fuming $37 \%$

Carl Roth

X942

InstantBlue-Protein Stain

Expedeon

ISB01L

IPTG (Isopropyl $\beta$-d-1-thiogalactopyranoside)

Sigma-Aldrich

15502

LDS Sample Buffer NuPAGE (4x)

Leupeptin Hemisulfat

Thermo Fisher Scientific

NP0007

Applichem

A2183

Lysozym

Applichem

A4972

Lysyl endopeptidase (LysC)

Methanol gradient grade for HPLC

Wako Chemicals

125-05061

VWR Chemicals

20864

Mini-PROTEAN TGX'm Precast Gels

$\mathrm{N}$-Ethylmaleimide (NEM)

Bio-Rad

4561085

Sigma-Aldrich

E3876

$\mathrm{NaCl}$

$\mathrm{NaN}_{3}$

Ni-NTA Magnetic Agarose Beads

Nonidet P40 (NP-40) BioChemica

Paraformaldehyde solution $4 \%$ in PBS

PBS Dulbecco w/o Ca/Mg 10x

PBS Dulbecco with $\mathrm{Ca} / \mathrm{Mg}$

Phenylmethyl sulphonyl fluoride (PMSF)

PhosSTOP Inhibitor Tablets

Sigma-Aldrich

31434

Applichem

A1430

Qiagen

36111

Applichem

A1694

Santa Cruz Biotechnology

sc-281692

Sigma-Aldrich

D1408

Sigma-Aldrich

D8662

Carl Roth

6367

Roche

4906845001

Thermo Fisher / Invitrogen

P36966

\section{DAPI}

Protein Marker VI (10-245) prestained

PVDF membrane Immobilon-FL, $0.45 \mu \mathrm{m}$

ReproSil pur $\mathrm{C}_{18}$ particles $1.9 \mu \mathrm{m}$

Applichem

A8889

MerckMillipore

IPFL00010

Dr. Maisch

LI-COR

926-11010

Applichem

A0675

SDS solution $20 \%$ for molecular biology

Sep-Pak $\mathrm{C}_{18}$ Cartridge

Waters

Skim Milk Powder

SnakeSkin Dialysis Tubing, 3.5 K MWCO

Sodium deoxycholate (Na-deoxycholate)

Sigma-Aldrich

70166

Thermo Fisher Scientific

88242

Applichem

A1531

Sodium dihydrogen phosphate monohydrate

$\left(\mathrm{NaH}_{2} \mathrm{PO}_{4}\right)$

Sodium Fluoride (NaF)

Carl Roth

K300

Applichem

A3904

Sodium hydroxide $(\mathrm{NaOH})$

Carl Roth

KK71

Spritzenfilter FiltropurS PES 33/0.45 $\mu \mathrm{m}$

Sarstedt

C2211

StrataClean Resin

Agilent

400714

Bio-Rad

1614023

Sigma-Aldrich

302031

Trifluoroacetic acid (TFA)

Carl Roth

AE15

Triton X-100

Carl Roth

3051 
Trypsin, sequencing grade modified

TUBE1 magnetic beads

Tween 20 for molecular biology

Urea

$\beta$-Mercaptoethanol
Promega

V5111

LifeSensors

Applichem

Applichem

Carl Roth
UM401M

A4974

A1049

4227

\subsubsection{Commercial kits}

\begin{tabular}{lll} 
Name & Company & $\begin{array}{l}\text { Catalogue } \\
\text { number }\end{array}$ \\
\hline In-Fusion HD Cloning Plus CE Kit & TaKaRa/Clontech & 638917 \\
Lysosome Enrichment Kit for Tissues and Cultured Cells & Thermo Scientific & 89839 \\
NucleoSpin Gel and PCR Clean-up Mini kit & Macherey-Nagel & 740609,5 \\
Plasmid Miniprep Kit I, peqGOLD & VWR PeqLab & $12-6942-02$ \\
PTMScan Ubiquitin Remnant Motif (K-E-GG) Kit & Cell Signaling & 5562 \\
PureYield Plasmid Maxiprep System & Promega & A2392 \\
Subcellular Protein Fractionation Kit for Cultured Cells & Thermo Scientific & 78840 \\
Venor GeM 100T \#VGM-100 & Minerva Biolabs & $11-1100$
\end{tabular}

\subsubsection{Cloning material}

\begin{tabular}{lll} 
Name & Company & $\begin{array}{l}\text { Catalogue } \\
\text { number }\end{array}$ \\
\hline Agarose LE GeneticPure & Biozym Scientific & 850070 \\
Ampicillin sodium salt & Carl Roth & K029.1 \\
Antarctic phosphatase & NEB & M0289 \\
BamHI & NEB & R3136 \\
BsmbI & NEB & R0739 \\
CutSmart buffer & NEB & \\
Deoxynucleotide Set, 100 mM & Sigma-Aldrich & DNTP100-1KT \\
Dimethyl sulfoxide (DMSO) & Applichem & A3006 \\
DNA Ladder 1 kb & New England Biolabs & N3232 \\
DpnI & NEB & R0176S \\
HindIII & NEB & R3104 \\
Kanamycin sulphate & Carl Roth & T832 \\
LB Agar (Luria/Miller) & Carl Roth & X969 \\
LB Broth (Luria/Miller) & Carl Roth & X968 \\
Notl & NEB & R3189 \\
Phusion High-Fidelity DNA polymerase incl. & NEB & M0530S \\
Phusion buffer & Carl Roth & 3865 \\
RotiSafe GelStain & NEB & M0202 \\
T4 DNA ligase & Thermo Fisher / & 10977035 \\
UltraPure DNase/RNase-Free distilled water & Invitrogen & \\
VELOCITY DNA Polymerase incl. VELOCITY buffer & bioline & BIO-21098 \\
Xbal & NEB & R0145 \\
Xhol & NEB & R0146
\end{tabular}




\subsubsection{Cell culture material}

\begin{tabular}{|c|c|c|}
\hline Name & Company & $\begin{array}{l}\text { Catalogue } \\
\text { number }\end{array}$ \\
\hline Bafilomycin A1 & LC Laboratories & B-1080 \\
\hline Dimethyl sulfoxide (DMSO) for cell culture & Applichem & A3672 \\
\hline DMEM for SILAC & $\begin{array}{l}\text { Life Technologies/ } \\
\text { Invitrogen }\end{array}$ & A33822 \\
\hline DMEM, high glucose, GlutaMAX Supplement & $\begin{array}{l}\text { Thermo Fisher Scientific / } \\
\text { Gibco }\end{array}$ & 61965 \\
\hline DMEM/F-12, GlutaMAX Supplement & $\begin{array}{l}\text { Thermo Fisher Scientific / } \\
\text { Gibco }\end{array}$ & 31331 \\
\hline EBSS, calcium, magnesium, phenol red & $\begin{array}{l}\text { Thermo Fisher Scientific / } \\
\text { Gibco }\end{array}$ & 24010 \\
\hline FBS South American & $\begin{array}{l}\text { Thermo Fisher Scientific / } \\
\text { Gibco }\end{array}$ & 10270106 \\
\hline Fetal Bovine Serum (FBS), dialyzed & $\begin{array}{l}\text { Thermo Fisher Scientific / } \\
\text { Gibco }\end{array}$ & 26400044 \\
\hline GeneJuice transfection reagent & Sigma-Aldrich & 70967 \\
\hline Hexadimethrine bromide (polybrene) & Sigma-Aldrich & H9268 \\
\hline Hygromycin B (50 mg/mL) & $\begin{array}{l}\text { Thermo Fisher Scientific / } \\
\text { Gibco }\end{array}$ & $10687-010$ \\
\hline INTERFERin siRNA transfection reagent & Polyplus Transfection & $409-10$ \\
\hline L-Arginin (R0) & Sigma-Aldrich & A8094 \\
\hline $\begin{array}{l}\text { L-Arginine hydrochloride (13C6, } 99 \% ; 15 N 4 \text {, } \\
99 \% \text { ) (R10) }\end{array}$ & $\begin{array}{l}\text { Cambridge Isotope } \\
\text { Laboratories }\end{array}$ & CNLM-539 \\
\hline L-Lysin (K0) & Sigma-Aldrich & L8662 \\
\hline $\begin{array}{l}\text { L-Lysine dihydrochloride (13C6, } 99 \% ; 15 N 2, \\
99 \% \text { ) (K8) }\end{array}$ & $\begin{array}{l}\text { Cambridge Isotope } \\
\text { Laboratories }\end{array}$ & CNLM-291 \\
\hline Lipofectamine 2000 & Invitrogen & 11668019 \\
\hline Lipofectamine RNAiMAX & Invitrogen & 13778075 \\
\hline MG132 (Z-Leu-Leu-Leu-CHO) & Sigma-Aldrich & $\mathrm{C} 2211$ \\
\hline Mr. Frosty freezing container & Thermo Fisher Scientific & $5100-0001$ \\
\hline Neubauer improved counting chamber & marienfeld superior & 640010 \\
\hline Non-Essential Amino Acids Solution (100x) & $\begin{array}{l}\text { Thermo Fisher Scientific / } \\
\text { Gibco }\end{array}$ & 11140050 \\
\hline Opti-MEM I Reduced Serum Medium & $\begin{array}{l}\text { Thermo Fisher Scientific / } \\
\text { Gibco }\end{array}$ & 31985 \\
\hline PBS Dulbecco w/o Ca/Mg & $\begin{array}{l}\text { Thermo Fisher Scientific / } \\
\text { Gibco }\end{array}$ & 14190 \\
\hline Penicillin-Streptomycin solution & Sigma-Aldrich & P4333 \\
\hline Polyethylenimine, linear & Polysciences Europe & $23966-2$ \\
\hline Puromycin dihydrochloride & Sigma-Aldrich & P8833 \\
\hline Sodium Pyruvate (100 mM) & $\begin{array}{l}\text { Thermo Fisher Scientific / } \\
\text { Gibco }\end{array}$ & 11360 \\
\hline Trypsin-EDTA (0.05 \%), phenol red & $\begin{array}{l}\text { Thermo Fisher Scientific / } \\
\text { Gibco }\end{array}$ & 25300 \\
\hline
\end{tabular}

\subsubsection{Mammalian cell lines and bacteria}

\begin{tabular}{|c|c|c|}
\hline Name & Cell line & Source \\
\hline HEK293 & human embryonic kidney & $\begin{array}{l}\text { German Collection of Microorganisms and Cell } \\
\text { Culture (DSMZ) }\end{array}$ \\
\hline HEK293T & & $\begin{array}{l}\text { kind gift from Ernst Stelzer (Institute for Cell } \\
\text { Biology and Neuroscience, Goethe University } \\
\text { Frankfurt) }\end{array}$ \\
\hline MCF7 & $\begin{array}{l}\text { breast cancer, Michigan } \\
\text { Cancer Foundation-7 }\end{array}$ & kind gift from Ernst Stelzer \\
\hline
\end{tabular}




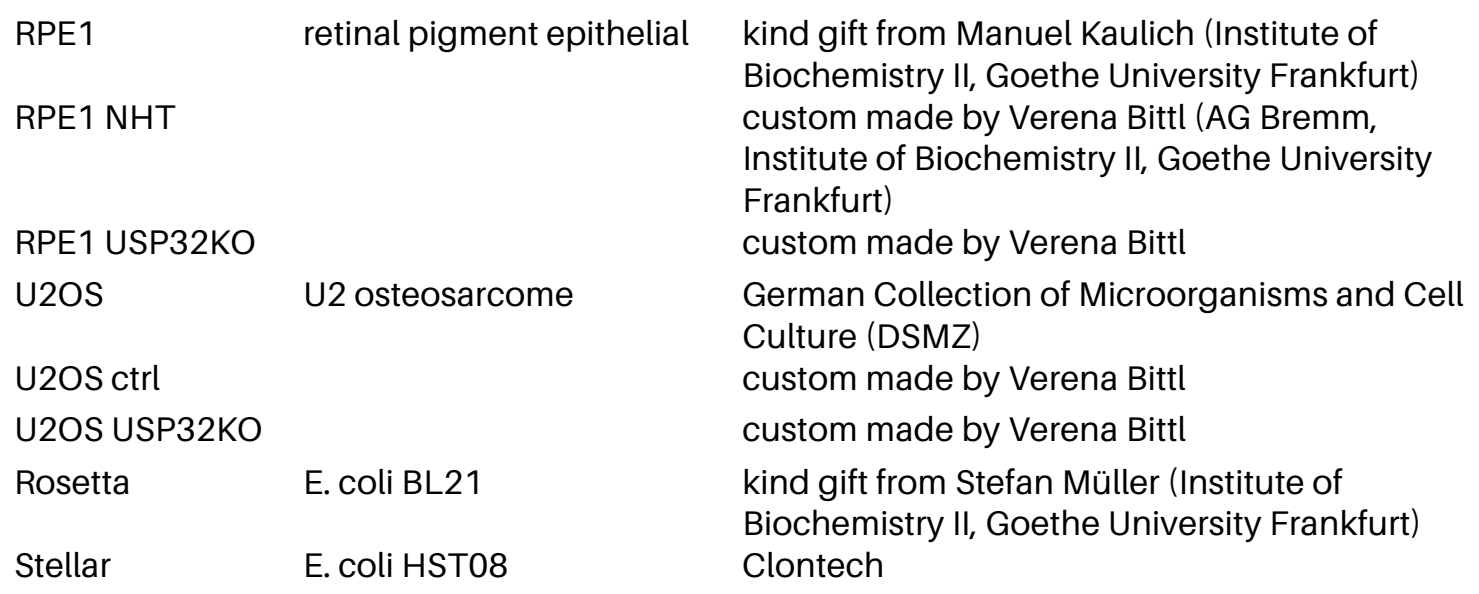

\subsubsection{Plasmids}

\begin{tabular}{l} 
Name \\
\hline pEGFPN1-USP32 (wt) \\
pEGFPN1-USP32 (362-1604) \\
pEGFPN1-USP32 (732-1604) \\
pEGFPN1-USP32 (1-610) \\
pEGFPN1-USP32 (1-733) \\
pEGFPC1-USP32 (wt) \\
pEGFPC1-GW-USP32 (wt) \\
pEGFPC1-GW-USP32 (732-1604) \\
pEGFPC1-GW-USP32 (1-610) \\
pEGFPC1-GW-USP32 (1-733) \\
pEGFPC1-GW-USP32 (362-1604) \\
pIRES-HA-USP32 (wt) \\
pIRES-HA-USP32 C743S \\
pAcGFP-C1-Rab7A \\
pCDNA3-Myc3-Rab7A
\end{tabular}

pGEX-RILP

PDEST-FLAG-HA-VPS26a

PDEST-Myc-VPS29

PDEST-FLAG-HA-VPS35

pEGFPC1

pEGFPN1

pEGFPN1-LAMTOR1

PEGFPN1-LAMTOR1 K20R
Source

USP32 cDNA as PCR template, subcloned in PEGFPN1 with BamHI restriction site

USP32 cDNA as PCR template, subcloned in PEGFPN1 with BamHI restriction site

USP32 cDNA as PCR template, subcloned in pEGFPN1 with BamHI restriction site

USP32 cDNA as PCR template, subcloned in PEGFPN1 with BamHI restriction site

USP32 cDNA as PCR template, subcloned in PEGFPN1 with BamHI restriction site

pEGFPN1-USP32 (wt) as PCR template, subcloned in PEGFPC1 with Xhol restriction site

pEGFPC1-USP32 (wt) as PCR template, subcloned in PEGFP-GW-JJ (linearized by PCR without USP32 ORF) pEGFPC1-USP32 (wt) as PCR template, subcloned in pEGFP-GW-JJ (linearized by PCR without USP32 ORF) pEGFPC1-USP32 (wt) as PCR template, subcloned in pEGFP-GW-JJ (linearized by PCR without USP32 ORF) pEGFPC1-USP32 (wt) as PCR template, subcloned in PEGFP-GW-JJ (linearized by PCR without USP32 ORF) pEGFPC1-USP32 (wt) as PCR template, subcloned in PEGFP-GW-JJ (linearized by PCR without USP32 ORF) pEGFP-GW-USP32 (wt) as PCR template, subcloned in pIRES-HA-C1 with Notl restriction site

site directed mutagenesis with pIRES-HA-USP32 (wt) as PCR template purchased from Addgene \#61803

PAcGFP-C1-Rab7A as PCR template, subcloned in pcDNAMyc3-Nrf2 (Addgene \#21555) by exchanging the Nrf2 ORF with $\mathrm{BamHI}$ and $\mathrm{Xbal}$ restriction site

kind gift from Ivan Dikic (Institute of Biochemistry II, Goethe University Frankfurt)

kind gift from Ivan Dikic

kind gift from Ivan Dikic

kind gift from Ivan Dikic

kind gift from Ivan Dikic

kind gift from Ivan Dikic

purchased from Addgene \#42334

site directed mutagenesis with pEGFPN1-LAMTOR1 as 


\author{
pEGFPN1-LAMTOR1 G2A \\ BSSK-8xHA-ubiquitin \\ BSSK-8xHis-ubiquitin \\ USP32 cDNA clone (ID: 6502630) \\ pEGFP-GW-JJ-USP32 \\ pIRES-HA-C1 \\ pcDNA3-HA-YWHAB \\ pLentiCRISPRv2
}

PCR template

pRK5-p18G2A-HA (Addgene \#42338) as PCR template, subcloned in pEGFPN1 with HindIII restriction site kind gift from Stefan Müller (Institute of Biochemistry II, Goethe University Frankfurt) kind gift from Stefan Müller

kind gift from Sylvie Urbe (Institute of Translational Medicine, University of Liverpool) introduction of HA ORF in pIRES-C1 by PCR with overlapping primers purchased from Addgene \#13270 kind gift from Feng Zhang, Addgene \#52961

\subsection{9 siRNA, gRNA, oligos}

\begin{tabular}{llll} 
Name & Company/application & $\begin{array}{l}\text { Catalogue } \\
\text { number }\end{array}$ & Sequence \\
\hline ON-TARGETplus USP32 & GE Healthcare/ & $\begin{array}{l}\text { J-006080- } \\
07-0005\end{array}$ & ggagauauccuguggguua \\
Dharmacon & 1027281 & \\
AllStars Negative & QIAGEN & \\
Uontrol siRNA & (gRNA for CRISPR KO) & caccggaatgcacatgacaccacaa \\
USP32_KO-1-F & (gRNA for CRISPR KO) & aaactgtggtgtcatgtgcattcc \\
USP32_KO-2-F & (gRNA for CRISPR KO) & caccgccacgtggagcattcttcc \\
USP32_KO-2-R & (gRNA for CRISPR KO) & aaacggaaagaatgctccacgtggc \\
USP32_KO-3-F & (gRNA for CRISPR KO) & caccgcagttacgtgaatactacag \\
USP32_KO-3-R & (gRNA for CRISPR KO) & aaacctgtagtattcacgtaactgc \\
USP32 seq1 & (sequencing USP32) & & gcttcaaggtatttgatgttgacc \\
USP32 seq2 & (sequencing USP32) & ggctacatcattaacactagaagg \\
USP32 seq3 & (sequencing USP32) & & cccattaaagcttcggtggac \\
USP32 seq4 & (sequencing USP32) & ccctttacaggttacatcattgc
\end{tabular}

\subsubsection{Primary antibodies}

\begin{tabular}{llll} 
Name & \multicolumn{3}{c}{ Catalogue } \\
number & application (dilution) \\
\hline AMBRA1 (G-6) & Santa Cruz & sc-398204 & WB $(1: 1,000)$ \\
Cathepsin D & Cell Signaling & 2284 & WB $(1: 500)$ \\
Cathepsin X/Z/P & R\&D Systems & AF934-SP & WB $(1: 2,000)$ \\
EGF Receptor & Cell Signaling & 2232 & WB $(1: 10,000)$ \\
GFP (B-2) & Santa Cruz & sc-9996 & WB $(1: 2,500)$ \\
GM130 & BD Transduction Lab & 610822 & IF $(1: 100)$ \\
HA-Tag (6E2) & Cell Signaling & 2367 & WB $(1: 2,000)$ \\
HIS-Probe & Santa Cruz & sc-53073 & WB $(1: 1,000)$ \\
Histone H2B (D2H6) & Cell Signaling & 12364 & WB $(1: 1,000)$ \\
Lamp-1 & DSHB & H4A3-c & WB $(1: 1,000)$, IF $(1: 200)$ \\
Lamp-2 & DSHB & H4B4-c & IF $(1: 200)$ \\
LAMTOR1 (D11H6) & Cell Signaling & 8975 & WB $(1: 1,000)$, IF $(1: 100)$ \\
LC3A/B (D3U4C) XP & Cell Signaling & 12741 & WB $(1: 1,500)$, IF $(1: 200)$ \\
M6PR, cation & Abcam & ab124767 & IF $(1: 200)$ \\
independent & & &
\end{tabular}




\begin{tabular}{|c|c|c|c|}
\hline mTOR (7C10) & Cell Signaling & 2983 & IF $(1: 200)$ \\
\hline Myc-Tag (9B11) & Cell Signaling & 2276 & WB $(1: 1,500)$ \\
\hline Normal Rabbit IgG & Cell Signaling & 2729 & endogenous IP \\
\hline p-Ulk1 (S757) & Cell Signaling & 6888 & WB $(1: 1,000)$ \\
\hline PARP & Cell Signaling & 9542 & WB $(1: 1,000)$ \\
\hline phospho-AMBRA1 Ser52 & Merck-Millipore & ABC80 & WB (1:500) \\
\hline Phospho-WIPI2 Ser413 & Cell Signaling & 13571 & WB $(1: 1,000)$ \\
\hline Rab11 (D4F5) & Cell Signaling & 5589 & WB $(1: 2,000)$ \\
\hline Rab7 (D95F2) XP & Cell Signaling & 9367 & WB $(1: 1,000)$, IF (1:100) \\
\hline TGN46 & Sigma & T7576 & IF (1:400) \\
\hline TMEM192 & custom made & $\begin{array}{l}\text { kind gift from } \\
\text { Paul Saftig } \\
\text { (Christian- } \\
\text { Albrechts- } \\
\text { University Kiel) }\end{array}$ & WB $(1: 2,000)$ \\
\hline Tubulin & Cell Signaling & 2125 & WB $(1: 2,000)$ \\
\hline Ubiquitin & Cell Signaling & 3933 & WB $(1: 1,000)$ \\
\hline ULK1 (D8H5) & Cell Signaling & 8054 & WB $(1: 1,000)$ \\
\hline USP32 & Sigma & HPA044365 & WB $(1: 1,500)$, IF $(1: 200)$ \\
\hline Vinculin & Sigma & V4505 & WB $(1: 10,000)$ \\
\hline VPS26A & Sigma & AMAb90967 & WB $(1: 1,500)$ \\
\hline VPS29 & Atlas Antibodies & HPA039748 & WB $(1: 1,500)$ \\
\hline VPS35 & Abcam & ab10099 & WB $(1: 1,000)$ \\
\hline WIPI2 (2A2) & Abcam & ab105459 & WB $(1: 1,000)$ \\
\hline
\end{tabular}

\subsubsection{Secondary antibodies}

\begin{tabular}{llll} 
& \multicolumn{3}{c}{ Catalogue } \\
Name & Company & number & application (dilution) \\
\hline anti-mouse IgG $(\mathrm{H}+\mathrm{L})$, Alexa Fluor 647 & Life Technologies & A-21235 & IF (1:200) \\
anti-rabbit IgG $(\mathrm{H}+\mathrm{L})$, Alexa Fluor 488 & Life Technologies & A-21206 & IF (1:200) \\
Anti-rabbit IgG, HRP-linked Antibody & Cell Signaling & $7074 S$ & WB (1:5000) \\
Anti-mouse IgG, HRP-linked Antibody & Cell Signaling & $7076 \mathrm{~S}$ & WB (1:5000)
\end{tabular}

\subsubsection{Buffers}

\begin{tabular}{|c|c|}
\hline Buffer & Ingredients \\
\hline BSA-PBS-T & $2.5 \%$ BSA, $0.05 \%$ Tween 20 in PBS \\
\hline DNA loading dye $6 x$ & $\begin{array}{l}10 \text { mM Tris-HCl (pH 7.6), } 0.03 \text { \% Bromphenol blue, } 60 \text { \% } \\
\text { Glycerol, } 60 \text { mM EDTA }\end{array}$ \\
\hline Lower Tris buffer $4 \mathrm{x}$ & 1.5 M Tris, $0.4 \%$ SDS (w/v), pH 8.8 \\
\hline PBS-T & $0.05 \%$ Tween 20 in PBS \\
\hline Running buffer $10 x$ & 250 mM Tris, 1.92 M Glycine, 1 \% SDS, pH 8.3 \\
\hline TEA buffer $10 x$ & 400 mM Tris, 200 mM Acetic acid, 10 mM EDTA, pH 8.4 \\
\hline TBS 10x & $500 \mathrm{mM}$ Tris, $1.5 \mathrm{M} \mathrm{NaCl}, \mathrm{pH} 7.5$ \\
\hline TBS-T & $0.1 \%$ Tween 20 in TBS \\
\hline Transfer buffer 10x & 250 mM Tris, 1.92 M Glycine \\
\hline Upper Tris buffer $4 \mathrm{x}$ & 0.5 M Tris, $0.4 \%$ SDS, pH 6.8 \\
\hline Antibody solution & $2 \% \mathrm{BSA}, 0.05 \% \mathrm{NaN}_{3}$ in TBS-T \\
\hline
\end{tabular}


RIPA

GFP-trap IP lysis buffer GFP-trap IP dilution buffer endogenous IP lysis buffer His pulldown lysis buffer His pulldown wash buffer $A$ His pulldown wash buffer $B$ TUBE pulldown lysis buffer TUBE pulldown wash buffer GST-RILP PD lysis buffer GST-RILP PD wash buffer TE buffer inhibitors for all lysis buffers or

Bacteria lysis buffer GSTRILP purification Low salt TBS High salt TBS GST-RILP dialysis buffer GST-RILP elution buffer

STAGE tip loading buffer

Solution A

Solution B

Urea buffer

Gel loading buffer

modified RIPA

SDC elution buffer

SDS separating gel

SDS stacking gel
$50 \mathrm{mM}$ Tris, $150 \mathrm{mM} \mathrm{NaCl}, 1 \%$ NP-40, 0.5 \% Na-deoxycholate, $0.1 \%$ SDS, $25 \cup$ Benzonase

$20 \mathrm{mM}$ Tris, $150 \mathrm{mM} \mathrm{NaCl}, 0.5 \%$ Triton X-100

$20 \mathrm{mM}$ Tris, $150 \mathrm{mM} \mathrm{NaCl}$

$20 \mathrm{mM}$ Tris, $150 \mathrm{mM} \mathrm{NaCl}, 0.5 \%$ Triton X-100

$6 \mathrm{M}$ Guanidin- $\mathrm{HCl}, 0.1 \mathrm{M} \mathrm{NaH}_{2} \mathrm{PO}_{4}, 0.05 \%$ Tween 20, $0.1 \mathrm{M}$ Tris, $\mathrm{pH} 8$

$8 \mathrm{M}$ Urea, $0.1 \mathrm{M} \mathrm{NaH}_{2} \mathrm{PO}_{4}, 0.05 \%$ Tween $20,0.01 \mathrm{M}$ Tris, $\mathrm{pH} 8$

$8 \mathrm{M}$ Urea, $0.1 \mathrm{M} \mathrm{NaH}_{2} \mathrm{PO}_{4}, 0.05 \%$ Tween 20, $0.01 \mathrm{M}$ Tris, pH 6.4

$50 \mathrm{mM}$ Tris, $150 \mathrm{mM} \mathrm{NaCl}, 1 \mathrm{mM}$ EDTA, $1 \%$ NP-40, 10\% Glycerol $20 \mathrm{mM}$ Tris, $150 \mathrm{mM} \mathrm{NaCl}, 0.1 \%$ Tween 20

$20 \mathrm{mM}$ Tris, $150 \mathrm{mM} \mathrm{NaCl}, 0.5 \%$ Triton X-100, 1 mM MgCl 2

$20 \mathrm{mM}$ Tris, $150 \mathrm{mM} \mathrm{NaCl}$

$50 \mathrm{mM}$ Tris, $10 \mathrm{mM}$ EDTA, pH 7.0

$1.5 \mu \mathrm{M}$ Aprotinin, $100 \mu \mathrm{M}$ Leupeptin, $1 \mathrm{mM}$ PMSF, $10 \mathrm{mM} \mathrm{NaF}$, $20 \mathrm{mM}$ NEM

1x cOmplete EDTA-free protease inhibitors, 1x PhosStop phosphatase inhibitors, 20mM NEM

$270 \mathrm{mM}$ Sucrose, $50 \mathrm{mM}$ Tris, $\mathrm{pH}$ 8, sterile filtered

25 mM Tris, 200 mM NaCl, pH 8, 5 mM DTT

$25 \mathrm{mM}$ Tris, $500 \mathrm{mM} \mathrm{NaCl}, \mathrm{pH}$ 8, $5 \mathrm{mM}$ DTT

$50 \mathrm{mM}$ Tris, $150 \mathrm{mM} \mathrm{NaCl}, 5 \mathrm{mM}$ EDTA, $0.1 \%$ NP-40

$30 \mathrm{mM} / 50 \mathrm{mM}$ GSH in low salt TBS

$1 \%$ TFA in $5 \%$ ACN

$0.1 \%$ formic acid in ACN in water

$0.1 \%$ formic acid in $95 \% \mathrm{ACN}$

$6 \mathrm{M}$ Urea, $10 \mathrm{mM}$ Tris

$125 \mathrm{mM}$ Tris, $20 \%$ Glycerol, $4 \%$ SDS, $3.75 \%$ betamercaptoethanol, $20 \mathrm{mM}$ DTT, $0.04 \%$ Bromphenol blue $50 \mathrm{mM}$ Tris, $150 \mathrm{mM} \mathrm{NaCl}, 1 \mathrm{mM}$ EDTA, 1 \% NP-40, $0.1 \%$ Na-deoxycholate, $25 \mathrm{U}$ benzonase 2\%, Na-deoxycholate, $1 \mathrm{mM}$ TCEP, 4mM CAA, 50 mM Tris, pH 8.5

7-15\% acrylamide, $1 \times$ lower Tris buffer, $0.07 \%$ APS, $0.07 \%$ TEMED

$5 \%$ acrylamide, $1 x$ upper Tris buffer, $0.1 \%$ APS, $0.1 \%$ TEMED

\subsection{Methods}

\subsubsection{Molecular Cloning}

\subsubsection{Cloning procedure}

Subcloning of open reading frames (ORFs) into expression vectors was done using the InFusion cloning system (In-Fusion HD Cloning Kit, Clontech) according to manufacturer's 
instructions. Oligonucleotide primers were designed with the Snapgene software's build-in InFusion Cloning tool.

Site-directed mutagenesis (SDM) was performed using the QuickChange Method. Mutagenesis primers were generated with the online QuickChange Primer Design Tool from Agilent. All oligonucleotides were purchased from Sigma-Aldrich.

\subsubsection{Polymerase chain reaction}

Polymerase chain reaction (PCR) was used either for subcloning to amplify short DNA fragments as inserts and to generate a linearized vector backbone or for side-directed mutagenesis. PCR amplification mix was prepared according to the manufacturer's protocol with Phusion High-Fidelity or Velocity DNA Polymerase. Per reaction, $50 \mu \mathrm{l} \mathrm{mix} \mathrm{were} \mathrm{prepared} \mathrm{in}$ DNAse/RNAse free water with 10-50 ng template DNA, $0.5 \mu \mathrm{M}$ forward and reverse primer, 1 mM dNTPs, 2-5\% DMSO, 1 x polymerase buffer and 1 unit polymerase. The mix was subjected to PCR cycle as stated below. The elongation time was calculated with $1 \mathrm{~min} / \mathrm{kb}$ (kilobase) of PCR product for subcloning, or set to $5: 30 \mathrm{~min}$ or $10 \mathrm{~min}$ for SDM. The annealing step was performed as a temperature ramp with $+0.4^{\circ} \mathrm{C}$ increase per step to end with the annealing temperature of the primer oligo.

Example PCR cycle for subcloning

\begin{tabular}{llll}
$98^{\circ} \mathrm{C}$ & $2 \mathrm{~min}$ & & \\
\hline $98^{\circ} \mathrm{C}$ & $30 \mathrm{sec}$ & & \\
$56^{\circ} \mathrm{C}$ & $30 \mathrm{sec}$ & $35 \mathrm{x}$ & $+0.4^{\circ} \mathrm{C} /$ cycle \\
$72^{\circ} \mathrm{C}$ & $15 \mathrm{sec}$ & & \\
\hline $72^{\circ} \mathrm{C}$ & $5 \mathrm{~min}$ & \\
$4^{\circ} \mathrm{C}$ & $\infty$
\end{tabular}

Example PCR cycle for SDM

\begin{tabular}{llll}
$98^{\circ} \mathrm{C}$ & $2 \mathrm{~min}$ & & \\
\hline $98^{\circ} \mathrm{C}$ & $30 \mathrm{sec}$ & & \\
$64^{\circ} \mathrm{C}$ & $30 \mathrm{sec} \quad 20 \mathrm{x}$ & $+0.4^{\circ} \mathrm{C} /$ cycle \\
$72^{\circ} \mathrm{C}$ & $5: 30 \mathrm{~min}$ & & \\
\hline $72^{\circ} \mathrm{C}$ & $5 \mathrm{~min}$ & \\
$4^{\circ} \mathrm{C}$ & $\infty$
\end{tabular}

\subsubsection{Restriction digest}

For vector linearization, DNA was digested with restriction enzymes in $50 \mu$ reactions containing $2 \mu \mathrm{g}$ vector, $1 \times$ CutSmart buffer and 2 units restriction enzyme. After $1 \mathrm{~h}$ incubation at $37^{\circ} \mathrm{C}, 1$ unit Antarctic Phosphatase was added, and incubated again for $10 \mathrm{~min}$ at $37^{\circ} \mathrm{C}$.

For removal of methylated template DNA in SDM reactions, 2 units Dpnl were added after the $\mathrm{PCR}$ to the SDM mix and incubated for $2 \mathrm{~h}$ at $37^{\circ} \mathrm{C}$.

\subsubsection{Agarose gel electrophoresis}

PCR products as well as linearized vectors were separated by agarose gel electrophoresis (GE) in tris acetate EDTA (TAE) buffer system. GeneRuler $1 \mathrm{~kb}$ DNA ladder and samples were mixed with loading dye and loaded onto a $0.8 \%$ agarose gel. Gel electrophoresis was performed at $100 \mathrm{~V}$ until the dye front reached the end of the gel. The DNA bands were visualized and excised under UV light. DNA was extracted from the gel using the NucleoSpin Gel and PCR Clean-up Kit according to the manufacturer's protocol. For SDM, the reaction was purified 
without agarose GE with the same kit according to the manufacturer's protocol. The obtained DNA concentration was determined with the UV-VIS BioSpectrometer basic at $260 \mathrm{~nm} / 280 \mathrm{~nm}$.

\subsubsection{In-Fusion reaction}

Subcloning of ORFs in expression vectors was performed using the In-Fusion ${ }^{\circledR}$ HD Cloning Plus System according to the manufacturer's protocol. Molar ratios of insert:vector were calculated using the online In-Fusion molar ratio calculator from Takara Bio. Each cloning reaction was filled up to $5 \mu \mathrm{l}$ or $10 \mu \mathrm{l}$, and incubated for $15 \mathrm{~min}$ at $50^{\circ} \mathrm{C}$.

\subsubsection{Transformation, amplification, purification and sequence verification}

For amplification, Stellar competent bacteria were transformed with appropriate amounts of DNA according to the manufacturer's protocol. After transformation with subcloning or SDM products, bacteria were plated on selective Luria broth (LB) agar plates and incubated over night at $37^{\circ} \mathrm{C}$. Clones were picked to inoculate a culture for small scale purification (MiniPrep).

For amplification of DNA plasmids, transformed bacteria were used directly to inoculate a culture for large scale purification (MaxiPrep). The bacterial cultures containing the selective antibiotics were incubated shaking over night at $37^{\circ} \mathrm{C}$. The following day, Mini or MaxiPreps were done using the Plasmid Miniprep Kit from Qiagen or the PureYield Plasmid Maxiprep System from Promega according to the manufacturer's protocol. The obtained DNA concentration was determined with the UV-VIS BioSpectrometer basic at $260 \mathrm{~nm} / 280 \mathrm{~nm}$. Sequence verification of purified DNA was performed by Sanger sequencing from Microsynth Seqlab.

\subsubsection{Guide RNA design and CRISPR/Cas9 plasmid generation}

The design of guide RNA (gRNA) and generation of plasmids for CRISPR/Cas9 knockout cell lines was done by Verena Bittl (AG Bremm, Institute of Biochemistry II, Goethe University Frankfurt). USP32 knockout RPE1 cells were generated using the CRISPR/Cas9 technology. Guide RNA sequences targeting spCas9 to the genomic locus of USP32 (ID Ensembl ENSG00000170832) were designed according to (Doench et al., 2016). Specific overhangs for subsequent ligation into pLentiCRISPRv2 (gift from Feng Zhang, Addgene plasmid \#52961) were added to each guide (underlined):

USP32_KO-1-F: CACCGgaatgcacatgacaccacaa

USP32_KO-1-R: AAACttgtggtgtcatgtgcattc $\underline{C}$

USP32_KO-2-F: CACCGccacgtggagcattctttcC

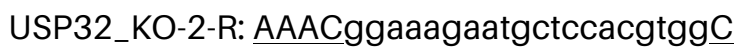

USP32_KO-3-F: CACCGcagttacgtgaatactacag

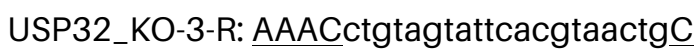

Complementary oligonucleotides were annealed for $5 \mathrm{~min}$ at $95^{\circ} \mathrm{C}$, and subsequently cooled down for $15 \mathrm{~min}$ at room temperature. Annealed primers were diluted to $0.5 \mu \mathrm{M}$ in nuclease free water and cloned into pLentiCRISPRv2 via BsmBI restriction enzyme digest and subsequent ligation with T4 DNA ligase. Stellar ${ }^{\text {rm }}$ competent bacteria were transformed with the ligation 
reaction and correct clones were identified by Sanger sequencing (Microsynth Seqlab) using the U6 primer.

\subsubsection{Mammalian cell culture}

\subsubsection{Cell maintenance}

hTERT RPE1 cells were cultured in DMEM/F-12 (Dulbecco's Modified Eagle Medium: Nutrient Micture F-12), GlutaMAX ${ }^{\mathrm{TM}}$ medium supplemented with $10 \%(\mathrm{v} / \mathrm{v})$ fetal bovine serum (FBS) $200 \mu \mathrm{g} / \mathrm{ml}$ Hygromycin B, $50 \mathrm{U} / \mathrm{ml}$ penicillin and $50 \mu \mathrm{g} / \mathrm{ml}$ streptomycin. U2OS, HEK293, and HEK293T cells were cultured in DMEM GlutaMAX ${ }^{\mathrm{TM}}$ medium supplemented with $10 \%(\mathrm{v} / \mathrm{v}) \mathrm{FBS}$ and $50 \mathrm{U} / \mathrm{ml}$ penicillin and $50 \mu \mathrm{g} / \mathrm{ml}$ streptomycin. MCF7 cells were cultured in DMEM GlutaMAX ${ }^{\mathrm{TM}}$ medium supplemented with $10 \%(\mathrm{v} / \mathrm{v})$ FBS, $50 \mathrm{U} / \mathrm{ml}$ penicillin and $50 \mu \mathrm{g} / \mathrm{ml}$ streptomycin, $1 \mathrm{mM}$ sodium pyruvate and $0.1 \mathrm{mM}$ non-essential amino acids. All cells were grown at $37{ }^{\circ} \mathrm{C}$ and $5 \% \mathrm{CO}_{2}$. The cells were subcultured at $80 \%$ confluency by washing with PBS, adding $0.05 \%$ Trypsin-EDTA to detach the cells and collecting in fresh medium. For regular maintenance, cells were seeded in a $10 \mathrm{~cm}$ dish in an appropriate estimated splitting ratio. For experiments, exact cell numbers were determined with a Neubauer counting chamber and cells were seeded in 6 well, $10 \mathrm{~cm}$ or $15 \mathrm{~cm}$ dishes.

PCR-based Mycoplasma contamination tests were regularly performed using the Venor ${ }^{\circledR} \mathrm{GeM}$ Classic kit.

\subsubsection{Cryopreserving and thawing cells}

For cryopreserving, detached cells were collected by centrifugation and resuspended in full cell culture medium containing $10 \%$ DMSO (dimethyl sulfoxide). The cryotubes were placed in isopropanol isolation container and frozen at $-80^{\circ} \mathrm{C}$. Ultimately, the cells were transferred to $150^{\circ} \mathrm{C}$ for long-term storage.

Cells were thawed at $37^{\circ} \mathrm{C}$ and resuspended full cell culture medium. To remove the DMSO, cells were collected by centrifugation, the medium was aspirated and cells were seeded in fresh medium to a dish.

\subsubsection{Transfection}

For DNA transfection, cells were seeded to achieve 30-40\% confluency the following day and transfected with PEI (polyethyleneimine, $25 \mathrm{kDa}$, linear, $1 \mathrm{mg} / \mathrm{ml}$, Polysciences Europe). Transfection mix was prepared with a DNA:PEI ratio 1:3 $(\mathrm{w} / \mathrm{w})$ for HEK293 cells and 1:5 for RPE1 cells in prewarmed Opti-MEM and incubated for $10 \mathrm{~min}$ at room temperature before adding to cells. RPE1 cells were transfected in medium without supplements, medium was exchanged with full medium after $4 \mathrm{~h}$. After transfection, cells were cultured for 18-24 $\mathrm{h}$ prior to lysis.

For siRNA transfection, U2OS, HEK293 and MCF7 cells were seeded to achieve 30-40\% confluency the following day and transfected with a final siRNA concentration of $30 \mathrm{nM}$ using INTERFERin according to the manufacturer's protocol. RPE1 cells were transfected with a final siRNA concentration of $20 \mathrm{nM}$ using Lipofectamine RNAiMAX following the manufacturer's 
reverse transfection protocol. The medium was exchanged after $24 \mathrm{~h}$ after transfection and cultured for additional $24 \mathrm{~h}$ prior to further treatment and/or lysis.

\subsubsection{Treatments}

Cells were starved for amino acids using EBSS (Earle's Balanced Salt Solution) medium for the indicated time points. Proteasomal inhibition was induced with $10 \mu \mathrm{M}$ MG132. Inhibition of lysosomal degradation was induced with $200 \mathrm{nM}$ Bafilomycin A1 (BafA1).

\subsubsection{Generation of high-titer lentivirus and transduction of cells}

The generation of high-titer lentivirus for CRISPR/Cas9 knockout cell lines was done by Verena Bittl (AG Bremm, Goethe University Frankfurt). $7.5 \times 10^{5}$ HEK293T cells were seeded into a 6well dish and cultivated in DMEM medium without antibiotics $24 \mathrm{~h}$ prior to transfection. Cells were transfected with Lipofectamine 2000 by mixing the reagent with $200 \mu$ Opti-MEM and $3.3 \mu \mathrm{g}$ transfer vector containing the gRNAs (pLentiCRISPRv2), $2.7 \mu \mathrm{g}$ PAX2 and $1 \mu \mathrm{g}$ pMD2.G. The transfection mix was incubated for $30 \mathrm{~min}$ at room temperature and added dropwise to HEK293T cells. Medium was replaced with fresh full DMEM $12 \mathrm{~h}$ after transfection. Supernatant containing lentiviral particles was collected after $24 \mathrm{~h}$ and $48 \mathrm{~h}$. Supernatants were pooled, aliquoted and frozen at $-80^{\circ} \mathrm{C}$.

For viral transduction, supernatants were thawed at room temperature, sterile filtered through $0.45 \mu \mathrm{m}$ filters and mixed with $10 \mu \mathrm{g}$ Polybrene (Sigma-Aldrich) to infect $1 \times 10^{6}$ RPE1 cells. Stable transduced cells were selected with puromycin and efficiency of USP32 knockout was confirmed by immunoblotting.

\subsubsection{Immunofluorescence microscopy}

\subsubsection{Staining}

For immunofluorescence microscopy, cells were grown on uncoated glass coverslips in 6 well plates (Greiner). Cells were fixed with cold $4 \%$ paraformaldehyde (PFA) solution in phosphatebuffered saline (PBS) for 15 min, washed twice with PBS and permeabilized with $1 \%$ Triton X100 in PBS for 15 min followed by a final wash with PBS. For LC3 staining, cells were fixed and permeabilized with ice-cold methanol $(\mathrm{MeOH})$ for $15 \mathrm{~min}$ at $-20^{\circ} \mathrm{C}$. Samples were blocked with $2.5 \%$ bovine serum albumin (BSA) in PBS with $0.05 \%$ Tween 20 (BSA-PBS-T) for 30 min. Primary antibodies were applied as indicated in the described dilution (6.1.10) in BSA-PBS-T for $1 \mathrm{~h}$ at room temperature (RT). Afterwards samples were washed three times for $5 \mathrm{~min}$ with PBS to remove residual antibody and incubated with corresponding fluorescently labeled secondary antibody in BSA-PBS-T for $1 \mathrm{~h}$ at RT. After additional washing with PBS (three time for $5 \mathrm{~min}$ ) cells were mounted using ProLong Diamond Antifade Reagent mounting medium with DAPI.

\subsubsection{CI-M6PR antibody-feeding assay}

Cl-M6PR internalization was observed using an antibody feeding assay in collaboration with Florian Steinberg (Center for Biological Systems Analysis, University of Freiburg). 
RPE1 cells on coverslips were washed once with cold PBS, incubated with cold full medium for 15 min at $4{ }^{\circ} \mathrm{C}$, and washed again with cold PBS. Afterwards, cells were incubated with CI-M6PR antibody in DMEM (dilution 1:100) for $1 \mathrm{~h}$ at $4^{\circ} \mathrm{C}$ followed by another wash with cold PBS. For internalization of the antibody, coverslips were transferred to prewarmed full medium and incubated at $37^{\circ} \mathrm{C}$ in the cell culture incubator for the indicated time points.

Subsequently, cells were fixed with PFA in PBS, stained with a specific primary antibody against TGN46 and corresponding fluorescently labeled secondary antibodies against the TGN46 and internalized CI-M6PR antibody as described in 6.2.3.1.

\subsubsection{DQ Red BSA assay}

U2OS or MCF7 cells were grown on uncoated glass coverslips in 6 well dishes. $10 \mu \mathrm{g} / \mathrm{ml} \mathrm{DQ}$ Red BSA was added for $1 \mathrm{~h}$. Cells were once washed with DMEM and incubated for $1 \mathrm{~h}$ or $2 \mathrm{~h}$ with full DMEM or EBSS. Afterwards, the cells were fixed with cold $4 \%$ PFA solution in PBS for 15 min, washed three times with PBS and mounted onto microscopy slides using ProLong Diamond Antifade Reagent mounting medium with DAPI.

\subsubsection{Imaging and Quantification}

Images were acquired on either a confocal Zeiss LSM780 or Leica SP8 LSM with a $63 \times$ oilimmersion objective in $512 \times 512$ or $1024 \times 1024$ scanning format using the standard Zeiss Zen or Leica LaX software. Color channels were saved separated per image in .tif file format for postcollection processing.

Quantification was performed with the open-source cell image analysis software CellProfiler (Carpenter et al., 2006). In brief, the pipeline for dot counting was build with the following sequential set of image analysis modules:

Table 6-1: CellProfiler pipeline for dot counting

\begin{tabular}{|c|c|}
\hline $\begin{array}{l}\text { Segmentation of nuclei in } \\
\text { DAPI channel image }\end{array}$ & $\begin{array}{ll}\text { - } & \text { Illumination correction (calculate and apply) } \\
\cdot & \text { smooth with Gaussian filter } \\
\text { - identify nuclei as primary object with Otsu thresholding } \\
\text { method }\end{array}$ \\
\hline $\begin{array}{l}\text { Segmentation of } \\
\text { cytoplasm in red channel } \\
\text { image }\end{array}$ & $\begin{array}{ll}\text {. } & \text { suppress features } \\
\text { - } & \text { smooth with Gaussian filter } \\
\text { identify cytoplasm as secondary objects with Otsu } \\
\text { thresholding method and nuclei as input object }\end{array}$ \\
\hline $\begin{array}{l}\text { Segmentation of dots in } \\
\text { green channel image }\end{array}$ & $\begin{array}{l}\text { enhance features } \\
\text { identify dots as primary objects with Otsu thresholding } \\
\text { method }\end{array}$ \\
\hline \multicolumn{2}{|c|}{ Export selected data to spreadsheet } \\
\hline
\end{tabular}

The pipeline for co-localization and dot dispersion was build with the following sequential set of image analysis modules:

Table 6-2: CellProfiler pipeline for co-localization and dot dispersion

\begin{tabular}{lll}
\hline Segmentation of nuclei in & $\cdot$ & $\begin{array}{l}\text { smooth with Gaussian filter } \\
\text { DAPI channel image }\end{array}$ \\
\hline
\end{tabular}


- identify nuclei as primary object with Otsu thresholding method

Segmentation of cytoplasm in red channel image

Segmentation of dots in red channel image

Segmentation of dots in green channel image
- suppress features

- smooth with Gaussian filter

- identify cytoplasm as secondary objects with Otsu thresholding method and nuclei as input object

- identify dots as primary objects with Otsu thresholding method

- identify dots as primary objects with Otsu thresholding method

Relate red dots to respective cytoplasm

Relate green dots to respective cytoplasm

Measure co-localization across entire red image and green image

Measure object intensity distributions within cytoplasm using nucleus as center across red (LAMP2) image

Export selected data to spreadsheet

Brightness and contrast were increased for all channels and conditions uniformly across the entire image using ImageJ (Schneider et al., 2012) where necessary for better visibility in the final figure.

\subsubsection{Statistics}

Results from repeat experiments were analyzed for statistical significance by a two-tailed, unpaired Student's t test between the indicated conditions using GraphPad Prism.

\subsubsection{Cell culture experiments}

\subsubsection{Cell lysis}

Unless described differently for specific experimental procedures, standard cell lysis was performed from a 6 well dish with $100 \mu$ RIPA buffer (containing phosphatase and protease inhibitors and nuclease) on ice for $10 \mathrm{~min}$. Lysates were transferred to a $1.5 \mathrm{ml}$ eppendorf tube. Soluble fractions were isolated by centrifugation for $10 \mathrm{~min}$ at $13,000 \mathrm{rpm}$ at $4{ }^{\circ} \mathrm{C}$. Cleared lysates were mixed with 4xLDS sample buffer supplemented with $20 \mathrm{mM}$ dithiothreitol (DTT) and boiled at $95^{\circ} \mathrm{C}$ for denaturation. Samples were subjected to SDS-PAGE and Western blot analysis.

\subsubsection{SDS-PAGE and immunoblotting}

Protein levels were analyzed by SDS (sodium dodecyl sulfate) polyacrylamide gel electrophoresis (PAGE) and Western blotting (WB) using the Mini-PROTEAN and Mini TransBlot system from Bio-Rad.

For SDS-PAGE, either self-made gels with acrylamide concentrations ranging from $7-15 \%$ or commercial precasted gels with a $4-15 \%$ gradient from Bio-Rad were used. Samples were loaded onto gels and separation was performed under constant voltage in Running buffer. 
Subsequently, proteins were transferred onto a methanol-activated polyvinylidene fluoride (PVDF) membrane (Immobilon FL, $0.45 \mu \mathrm{m}$ pore size) at constant $200 \mathrm{~mA}$ for $100 \mathrm{~min}$ in Transfer buffer. The membrane was blocked in $5 \%$ skim milk in TBS-T (tris-buffered salinetween 20) for $1 \mathrm{~h}$ at RT and afterwards washed with TBS-T to remove residual milk. The blot was incubated with primary antibody diluted in antibody solution over night at $4{ }^{\circ} \mathrm{C}$. After three times washing for $5 \mathrm{~min}$ with TBS-T at RT, the horse radish peroxidase (HRP)-coupled secondary antibody diluted in $5 \%$ skim milk in TBS-T was applied for $1 \mathrm{~h}$ at RT.

In every experiment (except immunoprecipitations and pulldowns), a house keeping protein like vinculin was blotted as loading control to ensure relative comparison between different conditions.

For chemiluminescence visualization, blots were incubated with ECL Prime Western Blot Detection Reagent and signal was detected with ChemiDocTM Imaging System (Biorad).

The acquired images were saved as original scn file. Adjustment of brightness and contrast of the image was done using the Image Lab software where necessary for better visibility in the final figure. Images were exported in .tif file format.

\subsubsection{Co-Immunoprecipitations}

\subsubsection{GFP-trap IP}

GFP-tagged proteins were immunoprecipitated (IP) from transfected cells using GFP-Trap Agarose beads from ChromoTek. $24 \mathrm{~h}$ after transfection, cells were lysed for $20 \mathrm{~min}$ at $4{ }^{\circ} \mathrm{C}$ with GFP IP lysis buffer (including PhosStop phosphatase inhibitors, cOmplete protease inhibitors and $20 \mathrm{mM}$ NEM (N-ethylmaleimide)). Depending on the used cell culture dish size, between $100 \mu \mathrm{l}$ and $1 \mathrm{ml}$ lysis buffer was used. Lysates were cleared by high-speed centrifugation at $13,000 \mathrm{rpm}$ for $10 \mathrm{~min}$ at $4{ }^{\circ} \mathrm{C}$ and transferred to a fresh tube. $10 \%$ of lysate was removed for the input sample and prepared for immunoblotting. Depending on the used cell culture dish size, 5$10 \mu \mathrm{l} \mathrm{GFP-Trap} \mathrm{beads} \mathrm{were} \mathrm{used} \mathrm{per} \mathrm{sample.} \mathrm{Beads} \mathrm{were} \mathrm{washed} \mathrm{twice} \mathrm{with} \mathrm{dilution} \mathrm{buffer} \mathrm{and}$ added to the lysates. Samples were incubated rotating for $1-3 \mathrm{~h}$ at $4{ }^{\circ} \mathrm{C}$. Beads were spun down at $3,500 \mathrm{xg}$ for $2 \mathrm{~min}$ at $4^{\circ} \mathrm{C}$ and washed three times with $500 \mu \mathrm{l}$ wash buffer. For later immunoblotting, the beads were resuspended in $40 \mu \mathrm{L} 2 \mathrm{xDS}$ (lithium dodecyl sulfate) sample buffer containing $20 \mathrm{mM}$ DTT and boiled for $10 \mathrm{~min}$ at $95^{\circ} \mathrm{C}$.

\subsubsection{IP of endogenous proteins}

Endogenous proteins were co-immunoprecipitated from cells using specific antibodies. Cells from a $10 \mathrm{~cm}$ dish were lysed for $20 \mathrm{~min}$ at $4{ }^{\circ} \mathrm{C}$ with $600 \mu$ lysis buffer (containing PhosStop phosphatase inhibitors and cOmplete protease inhibitors). Lysates were cleared by high-speed centrifugation at $13,000 \mathrm{rpm}$ for $10 \mathrm{~min}$ at $4{ }^{\circ} \mathrm{C}$ and transferred to a fresh tube. $10 \%$ of lysate was removed for the input sample and prepared for immunoblotting. SureBeads Protein $G$ Magnetic Beads (Bio-Rad) were washed three times with $500 \mu$ lysis buffer. A pre-clearing step of the lysates was performed with $15-20 \mu \mathrm{l}$ washed unloaded beads rotating for $1 \mathrm{~h}$ at $4{ }^{\circ} \mathrm{C}$. Afterwards, the samples were magnetized and the supernatant was transferred to a fresh tube. $5 \mu \mathrm{l} \mathrm{IgG}$ control or $10 \mu \mathrm{g}$ specific antibody were added to the pre-cleared lysates. Samples were 
incubated rotating at $4^{\circ} \mathrm{C}$ for $1 \mathrm{~h}$. 50-100 $\mu$ washed SureBeads were added and the IP samples were incubated additionally for $1 \mathrm{~h}$ at $4^{\circ} \mathrm{C}$. Subsequently, the beads were magnetized and washed three times with $500 \mu$ lysis buffer. Samples were eluted with $45 \mu$ l 2xLDS sample buffer for $10 \mathrm{~min}$ at $95^{\circ} \mathrm{C}$ and the supernatant was transferred to a fresh tube. Afterwards, $20 \mathrm{mM}$ DTT was added and boiled again for protein denaturation. Samples were subjected to immunoblotting.

\subsubsection{Pulldowns}

\subsubsection{His-ubiquitin pulldown}

Ubiquitination of proteins was assessed by co-expression with His- or HA-tagged (for control) ubiquitin in HEK293 cells. $48 \mathrm{~h}$ after transfection, cells were lysed for $15 \mathrm{~min}$ at $99^{\circ} \mathrm{C}$ with lysis buffer. Lysates were cleared by high-speed centrifugation at $13,000 \mathrm{rpm}$ for $10 \mathrm{~min}$ and transferred to a fresh tube. $10 \%$ of lysate was removed for input sample and prepared for immunoblotting by protein precipitation.

$25 \mu \mathrm{l} /$ sample Ni-NTA Magnetic Agarose Beads (Qiagen) were washed twice with $1 \mathrm{ml}$ lysis buffer and added to the cleared lysates. Pulldown (PD) was incubated overnight at RT while rotating. The next day, the beads were magnetized and washed three times with $750 \mu \mathrm{l}$ wash buffer A, then washed twice with $750 \mu \mathrm{l}$ wash buffer $B$. The last washing step was performed using $750 \mu \mathrm{l}$ PBS. The beads were resuspended in $80 \mu \mathrm{l} 2$ xLDS sample buffer containing $20 \mathrm{mM}$ DTT. Samples were boiled for $10 \mathrm{~min}$ at $95^{\circ} \mathrm{C}$ and subjected to immunoblotting.

The proteins in the input sample were precipitated with $10 \%$ trichloroacetic acid (TCA) and pelleted by high-speed centrifugation at $13,000 \mathrm{rpm}$ for $15 \mathrm{~min}$. The supernatant was removed and the protein pellet was resuspended in $200 \mu \mathrm{l} 100 \%$ ethanol (EtOH). Samples were centrifuged again for $20 \mathrm{~min}$ at $13,000 \mathrm{rpm}$. EtOH was removed and the pellet was dried by evaporation over night at RT. The input pellet was dissolved in $200 \mu \mathrm{L} 2$ xLDS sample buffer containing $20 \mathrm{mM}$ DTT. Samples were boiled for $10 \mathrm{~min}$ at $95^{\circ} \mathrm{C}$ and subjected to immunoblotting.

\subsubsection{TUBE pulldown}

Ubiquitinated proteins were enriched by using Tandem Ubiquitin Binding Entities (TUBEs). RPE1 cells were lysed for $20 \mathrm{~min}$ at $4{ }^{\circ} \mathrm{C}$ with TUBE PD lysis buffer (including Phosstop phosphatase inhibitors, cOmplete protease inhibitors and $20 \mathrm{mM} \mathrm{NEM}$ ). Lysates were cleared by highspeed centrifugation at $13,000 \mathrm{rpm}$ for $10 \mathrm{~min}$ at $4{ }^{\circ} \mathrm{C}$ and transferred to a fresh tube. $5 \%$ of lysate was removed for the input sample and prepared for immunoblotting. $40 \mu$ LUBE1 magnetic beads (LifeSensors) or Control magnetic beads (LifeSensors) were washed twice with $1 \mathrm{ml}$ wash buffer and added to the lysates. Pulldown (PD was incubated rotating overnight at $4^{\circ} \mathrm{C}$. The next day, beads were magnetized and the supernatant was removed. The samples were washed three times with $500 \mu \mathrm{l}$ cold wash buffer and afterwards resuspended in $40 \mu \mathrm{l}$ 2xLDS sample buffer containing $20 \mathrm{mM}$ DTT. Samples were boiled for $10 \mathrm{~min}$ at $95^{\circ} \mathrm{C}$ and subjected to immunoblotting. 


\subsubsection{GST-RILP pulldown}

Activity of Rab7 was investigated by its binding to the effector protein RILP. Therefore recombinant GST-RILP was purified and used to enrich Rab7 from RPE1 NHT and USP32KO cells. The experiment was performed in collaboration with Florian Steinberg (Center for Biological Systems Analysis, University of Freiburg).

\section{Expression and purification of recombinant GST-RILP}

Rosetta bacteria were transformed with pGEX-RILP and used to inoculate $100 \mathrm{ml}$ overnight culture with selection. The next morning, $2 x 1$ L LB medium with selection were inoculated with the overnight culture to have a starting OD600 of 0.1 . Cultures were grown shaking at $37^{\circ} \mathrm{C}$ until OD600 $=0.6$. The cultures were cooled down for $30 \mathrm{~min}$ at $4{ }^{\circ} \mathrm{C}$. Afterwards, protein production was induced with $250 \mu \mathrm{M}$ Isopropyl $\beta$-d-1-thiogalactopyranoside (IPTG) and cultures were grown overnight at $20^{\circ} \mathrm{C}$ shaking at $120 \mathrm{rpm}$. Bacteria were pelleted for $30 \mathrm{~min}$ at $4,000 \mathrm{rpm}$ at $4^{\circ} \mathrm{C}$. Following protein purification was performed on ice with cold buffers. Bacteria were lysed with $40 \mathrm{ml}$ lysis buffer containing a small amount of lysozyme (spatula's tip full), $1 \mathrm{mg} / \mathrm{ml}$ DNAse and 1 tablet complete protease inhibitors. The solution was sonicated briefly ( $3 \times 30 \mathrm{sec}$ with $5 \mathrm{sec}$ on $-5 \mathrm{sec}$ off at $40 \%$ amplitude) and bacteria were lysed in two passages using an Emulsiflex homogenisor (French press) until the lysate looked clear. Cell debris was pelleted for $30 \mathrm{~min}$ at $20,000 \mathrm{xg}$ at $4{ }^{\circ} \mathrm{C}$ and the suspension was filtered through a $0.45 \mu \mathrm{m}$ filter. For batch purification, $1 \mathrm{ml}$ glutathione (GSH) agarose beads slurry was pipetted into a $50 \mathrm{ml}$ glass chromatography column, washed twice with $2 \mathrm{ml}$ water and twice with $2 \mathrm{ml}$ lysis buffer (without inhibitors). Filtered supernatant was applied to the column and incubated on a roller for $2 \mathrm{~h}$ at $4{ }^{\circ} \mathrm{C}$. Afterwards, beads were washed with $10 \mathrm{ml}$ lysis buffer, $1 \mathrm{~L}$ high salt TBS, and $1 \mathrm{~L}$ low salt TBS. For elution of GST-RILP, beads were incubated $2 \mathrm{x}$ with $2 \mathrm{ml}$ followed by incubation with $2 \times 1 \mathrm{ml}$ elution buffer containing $30 \mathrm{mM} \mathrm{GSH}$. For the following last elution step, $1 \mathrm{ml}$ elution buffer with $50 \mathrm{mM}$ GSH was used and beads were incubated for $10 \mathrm{~min}$ on ice. The fractions with GST-RILP were collected.

Subsequent buffer exchange was achieved by dialysis. The eluted GST-RILP was filled in a SnakeSkin diaylsis tube according to manufacturer's protocol, put in $4 \mathrm{~L}$ dialysis buffer, and stirred overnight at $4^{\circ} \mathrm{C}$. After dialysis, proteins were concentrated using Amicon Ultra centrifugal filter units ( $3 \mathrm{kDa}$ cutoff) to have 1-2 $\mathrm{ml}$ end volume. Snap freeze aliquoted protein in liquid nitrogen and freeze at $-80^{\circ} \mathrm{C}$.

During the purification process, samples from bacterial pellet, supernatant after cell lysis and centrifugation, supernatant after incubation with beads, beads after washing, beads after elution, and from each elution fraction were taken for later process examination by SDS-PAGE.

\section{GST-RILP pulldown}

$30 \mu \mathrm{l}$ glutathione agarose beads were washed twice with $1 \mathrm{ml}$ wash buffer and loaded with $75 \mu \mathrm{g}$ GST-RILP for $2 \mathrm{~h}$ rotating at $4{ }^{\circ} \mathrm{C}$. RPE1 cells were lysed for $20 \mathrm{~min}$ at $4{ }^{\circ} \mathrm{C}$ with $500 \mu \mathrm{l} \mathrm{GST}$ RILP PD lysis buffer (including PhosStop phosphatase inhibitors, cOmplete protease inhibitors). Lysates were cleared by highspeed centrifugation at $13,000 \mathrm{rpm}$ for $10 \mathrm{~min}$ at $4{ }^{\circ} \mathrm{C}$ and transferred to a fresh tube. $10 \%$ of lysate were removed for the input sample and prepared for immunoblotting. Loaded GST-RILP beads were added to the lysates. Pulldown was incubated rotating for $2 \mathrm{~h}$ at $4{ }^{\circ} \mathrm{C}$. Beads were spun down at $300 \mathrm{xg}$ for $5 \mathrm{~min}$ at $4{ }^{\circ} \mathrm{C}$ and washed three 
times with $500 \mu \mathrm{l}$ wash buffer. For later immunoblotting, the beads were resuspended in $40 \mu \mathrm{l}$ 2xLDS sample buffer containing $20 \mathrm{mM}$ DTT and boiled for $10 \mathrm{~min}$ at $95^{\circ} \mathrm{C}$.

\subsubsection{Subcellular fractionation}

Subcellular fractionation of RPE1, U2OS and MCF7 cells was performed using the Subcellular Protein Fractionation Kit for Cultured Cells (Thermo Fisher Scientific) according to manufacturer's protocol by extraction with different buffers and subsequent centrifugation steps.

In brief, $1 \times 10^{6}$ cells were lysed using $100 \mu$ CEB buffer on ice. The cytoplasmic fraction was extracted by centrifugation at $500 \mathrm{xg}$. Extraction of the membrane fraction was performed with $100 \mu \mathrm{l}$ MEB buffer and centrifugation at 3,000 xg. The soluble nuclear fraction was obtained by adding $50 \mu \mathrm{l}$ NEB buffer, short vortexing, incubation on ice, and centrifugation at 5,000 xg. $50 \mu \mathrm{l}$ of the same buffer supplemented with $\mathrm{CaCl}_{2}$ and micrococcal nuclease were used for extraction of the chromatin bound fraction followed by high-speed centrifugation at 16,000 xg. Supernatants with the respective fraction were mixed with LDS running buffer and $20 \mathrm{mM}$ DTT and prepared for later SDS-PAGE and Western blot analysis.

\subsubsection{Lysosome purification}

Lysosomes were enriched with the Lysosome Enrichment Kit from Thermo Scientific according to the manufacturer's protocol with adjustments.

In brief, 50-200 mg cells were lysed with $800 \mu$ lysosome enrichment reagent A in a controlled way by shearing through a $27 \mathrm{G}$ needle. Lysis efficiency was monitored by spotting a small sample onto a glass slide and comparing with a whole cell control. $10 \%$ of the whole cell lysate were removed for the input sample and prepared for immunoblotting. The lysate was cleared by centrifugation and applied on a discontinuous OptiPrep density gradient in ultracentrifugation tubes. Ultracentrifugation was performed at $14,5000 \times \mathrm{xg}$ at $4{ }^{\circ} \mathrm{C}$ for $2 \mathrm{~h}$ with maximum acceleration and deceleration. The upper $2 \mathrm{ml}$ band containing the lysosome fraction was transferred to fresh tube and diluted with three volumes PBS. After pelleting the lysosome for $30 \mathrm{~min}$ with $18,000 \mathrm{rpm}$ at $4{ }^{\circ} \mathrm{C}$, the supernatant was aspirated. The lysosomal pellet was washed and dissolved in $40 \mu \mathrm{L} 2 \times \mathrm{LDS}$ with $20 \mathrm{mM}$ DTT. Samples were boiled for $3 \mathrm{~min}$ at $95^{\circ} \mathrm{C}$ and subjected to SDS-PAGE and Western Blot or LC-MS/MS (liquid chromatography-mass spectrometry/mass spectrometry) analysis.

\subsubsection{Enrichment of secreted proteins}

Secreted proteins were enriched form the cell culture supernatant with two different approaches. For both methods, U2OS or RPE1 cells were grown in 6 well or $10 \mathrm{~cm}$ dishes. Medium was exchanged with serum-free Opti-MEM and conditioned for $24 \mathrm{~h}$. The Opti-MEM was transferred to a fresh tube. Residual cells were pelleted by centrifugation for 6 min at $1,000 \mathrm{xg}$. For the input sample, the cells in the dish were lysed directly in $2 x$ LDS containing $20 \mathrm{mM}$ DTT. 
Enrichment with Amicon Ultra centrifugal filter units was performed as follows: an Amicon ${ }^{\circledast}$ Ultra-0.5 Ultracel-3 membrane ( $3 \mathrm{kDa}$ cutoff) was equilibrated with $500 \mu \mathrm{l}$ Opti-MEM for $3 \mathrm{~min}$ at $14,000 \mathrm{xg}$. The supernatant was loaded into Amicon unit in $500 \mu \mathrm{l}$ steps and centrifuged for 5$7 \mathrm{~min}$ at $14,000 \mathrm{xg}$. In between, the sample was mixed carefully by pipetting up and down. The concentration of the protein solution was finished when a final volume of $100 \mu \mathrm{l}$ was reached. The sample was recovered by placing the Amicon filter unit upside down in a clean tube and spinning for $2 \mathrm{~min}$ at $1,000 \mathrm{xg}$. Protein concentration was measured using a Bradford assay. The recovered concentrate was mixed with LDS running buffer and $20 \mathrm{mM}$ DTT and prepared for later SDS-PAGE and Western blot analysis.

Enrichment with StrataClean beads was performed according to Bonn 2014 as follows: $20 \mu \mathrm{l} / \mathrm{sample}$ StrataClean resin (Agilent) were pelleted for $2 \mathrm{~min}$ at 6,000 $\mathrm{xg}$ and washed twice with $500 \mu \mathrm{l}$ TE buffer. For cleaning and hydrolyzing all impurities which may be loaded on the beads $200 \mu \mathrm{l}$ concentrated $\mathrm{HCl}$ was added and incubated overnight at $100^{\circ} \mathrm{C}$ under the fume hood. Beads were sedimented for $2 \mathrm{~min}$ at $6,000 \mathrm{xg}$, washed twice with $1 \mathrm{ml}$ tris-EDTA (TE) buffer and added to the cleared supernatants. Samples were incubated rotating overnight at $4{ }^{\circ} \mathrm{C}$. The loaded beads were pelleted by centrifugation for 20 min with $10,000 \times g$ at $4{ }^{\circ} \mathrm{C}$. Supernatant was discarded. Beads were washed twice with TE buffer and dissolved in $40 \mu \mathrm{l}$ $2 \times$ LDS with $20 \mathrm{mM}$ DTT. Samples were boiled for $3 \mathrm{~min}$ at $95^{\circ} \mathrm{C}$ and subjected to SDS-PAGE and Western Blot.

\subsubsection{Mass spectrometry experiments}

\subsubsection{1 diGly IP}

The ubiquitinome analysis was done with a SILAC based diGly remnant profiling approach. RPE1 NHT and USP32KO cells were grown for two weeks in DMEM suitable for SILAC containing either light (KORO) or heavy (K8R10) lysine and arginine.

The diGly remnant profiling protocol was established by the lab of Petra Beli (Institute of Molecular Biology, Mainz).

\section{Protein precipitation}

Cells were lysed for 15 min on ice with modified RIPA lysis buffer (including PhosStop phosphatase inhibitors cOmplete protease inhibitors, and $20 \mathrm{mM} \mathrm{NEM}$ ). $5 \mathrm{M} \mathrm{NaCl}$ ( $10 \%$ volume of the lysate volume) was added, and lysates were sonicated and cleared by centrifugation. Protein concentration was measured using Bradford and lysates from heavy and light labeled samples were combined in a 1:1 ratio. $50 \mu \mathrm{g}$ protein of the pooled sample were removed for later full proteome analysis for normalization. Proteins were precipitated in $80 \%$ ice-cold acetone at $-20^{\circ} \mathrm{C}$ over night.

\section{In-solution digest}

The next day, the precipitated proteins were pelleted by centrifugation, and washed with $70 \%$ $\mathrm{EtOH}$. The dried pellets were dissolved in denaturation buffer and subjected to in-solution digest. Proteins were reduced with $1 \mathrm{mM}$ DTT for $45 \mathrm{~min}$ at RT and alkylated with $5 \mathrm{mM}$ chloroacetamide (CAA) for $30 \mathrm{~min}$ at RT in the dark. Samples were digested with $1 \mu \mathrm{g} / \mu \mathrm{L} \mathrm{LySC}$ 
for every $200 \mu \mathrm{g}$ protein for $4 \mathrm{~h}$ and subsequently with $1 \mu \mathrm{g} / 200 \mu \mathrm{g}$ protein trypsin over night at RT. The digest was stopped with $0.5 \%$ trifluoroacetic acid (TFA).

\section{Sep-Pak purification of peptides}

Peptides were purified on $\mathrm{C}_{18}$ Sep-Pak columns. The columns were washed once with $100 \%$ acetonitrile (ACN) and then three times with $0.1 \%$ TFA. Peptides were loaded on the columns and washed three times with water. The peptides were eluted with $50 \% \mathrm{ACN}$.

\section{diGly remnant immunoaffinity purification}

The immunoaffinity purification (IAP) for ubiquitinated peptides was performed using the PTMScan Ubiquitin Remnant Motif (K-e-GG) Kit from Cell Signaling. 10x IAP buffer was added to the purified peptides and samples were concentrated to $1 \mathrm{ml}$ by SpeedVac vacuum centrifugation.

PTMScan Ubiquitin Remnant Motif (K-e-GG) Antibody beads were washed three times with IAP buffer. Peptides were added and incubated rotating for $4 \mathrm{~h}$ at $4{ }^{\circ} \mathrm{C}$. The beads were spun down and washed two times with IAP buffer containing $150 \mathrm{mM} \mathrm{NaCl}$ and $0.5 \% \mathrm{NP}-40$, two times with IAP buffer only and two times with water. Peptides were eluted from beads in a four step procedure using $0.15 \%$ TFA. Supernatants were combined into a fresh tube.

\section{Micro SCX fractionation}

The IAP eluents were fractionated using SCX STAGE tips and elution in six fractions with different $\mathrm{pH}$ steps. The STAGE tips were washed once with $\mathrm{MeOH}$, once with the lowest and the highest $\mathrm{pH}$ buffer and then with water. After loading the peptides onto SCX STAGE tips, samples were washed with $0.1 \%$ TFA in $40 \% \mathrm{ACN}$ and eluted with increasing $\mathrm{pH}$ of elution buffer ( $\mathrm{pH}$ steps 4.0, 5.0, 6.0, 7.0, 8.5, 11.0). Samples were concentrated by SpeedVac vacuum centrifugation and acidified with STAGE tip loading buffer.

\section{In-gel digest of proteome samples}

For the full proteome analysis, $50 \mu \mathrm{g}$ protein sample were mixed with LDS and $10 \mathrm{mM}$ DTT and heated for $10 \mathrm{~min}$ at $70^{\circ} \mathrm{C} .55 \mathrm{mM} \mathrm{CAA}$ was added and samples were separated on a $10 \%$ SDSPAGE followed by Coomassie protein staining with InstantBlue (Expedeon). Gel lanes were cut into equal pieces and subjected to in-gel digestion. Gel pieces were destained three times for 15 min at RT with $40 \% \mathrm{EtOH}$ in $50 \mathrm{mM}$ ammonium bicarbonate $(\mathrm{ABC})$, and subsequently dehydrated in $100 \%$ EtOH two times for $5 \mathrm{~min}$ at RT. The supernatant was removed and the gel pieces were dried by SpeedVac vacuum centrifugation. The proteins were reduced with $10 \mathrm{mM}$ DTT for $45 \mathrm{~min}$ at $60^{\circ} \mathrm{C}$ and alkylated with $55 \mathrm{mM}$ CAA for $30 \mathrm{~min}$ at RT in the dark. The gel pieces were again washed and destained with $40 \% \mathrm{EtOH}$ in $50 \mathrm{mM} \mathrm{ABC}$, and subsequently dehydrated in $100 \% \mathrm{EtOH}$. The supernatant was removed and the gel pieces were dried by SpeedVac vacuum centrifugation. The samples were rehydrated with digestion buffer containing $5 \mathrm{ng} / \mathrm{\mu l}$ Trypsin and incubated over night at $37^{\circ} \mathrm{C}$. Elution of peptides was performed using an increasing ACN concentration from $30 \%$ to $100 \%$. The supernatants were combined and concentrated by SpeedVac vacuum centrifugation. Samples were acidified with STAGE tip loading buffer.

\section{$\mathrm{C}_{18}$ STAGE tip purification}

Purification and concentration for proteome and IAP samples was performed using $\mathrm{C}_{18}$ STAGE tips. The in-house assembled $\mathrm{C}_{18}$ STAGE tips were activated with $\mathrm{MeOH}$ and subsequently 
equilibrated with STAGE tip loading buffer. Peptides in STAGE tip loading buffer were loaded on the STAGE tips and washed with $0.5 \%$ acetic acid (AA). Elution was performed with $80 \%$ $A C N$ in $0.5 \% A A$ and peptides were dried afterwards by SpeedVac vacuum centrifugation. For LC-MS/MS analysis, peptides were rehydrated in $0.1 \%$ TFA and $2 \%$ ACN.

\section{LC-MS/MS analysis}

The analysis by liquid chromatography and mass spectrometry was done by Florian Bonn (Institute of Biochemistry II, Goethe University Frankfurt)

Peptides were loaded with an easy nLC1200 onto a self-made $15 \mathrm{~cm}$ column filled with $1.7 \mu \mathrm{m}$ $\mathrm{C}_{18}$ particles. For the analysis of peptides obtained by diGly enrichment, the peptides were separated with a 58 minutes gradient from 10 to $38 \%$ B (B being $0.1 \%$ formic acid in $95 \%$ acetonitrile and $A$ being $0.1 \%$ formic acid in acetonitrile in water.). The eluting peptides were directly injected into a $\mathrm{Q}$ Exactive HF operating in DDA mode. In brief, after a survey scan 60,000 resolution the 10 most abundant precursor ions were fragmented by HCD and the resulting fragments analyzed with 30,000 resolution. Only precursors with charge states 3-7 were taken into account for fragmentation and afterwards dynamically excluded for $20 \mathrm{sec}$. After the gradient, the column was washed with $100 \% \mathrm{~B}$ and reequilibrated for a total analysis time of 75 minutes. For analysis of peptides prepared by the gel-based approach, the analysis was the same, but the number of precursor ions chosen for fragmentation (15) and that also precursor ions with a charge of 2 were subjected to further analysis.

\section{Mass spectrometry data processing}

Data analysis was done with MaxQuant 1.5.1 against the Uniprot Human Reference Proteome database with standard settings and activated SILAC quantification (K8). For analysis of the samples from the diGly approach, diGly modification of lysines were set as an additional variable modification. Ratios for peptides with diGly modification were adjusted to total protein level by correction with the data from the total protein experiment. Differentially abundant peptides harboring a diGly motif $(p<0.01)$ were detected with a One-sided T-Test with Perseus.

\subsubsection{Lysosome proteome}

The proteome analysis of purified lysosomes was done with a SILAC based approach. U2OS ctrl and USP32KO cells were grown for two weeks in DMEM suitable for SILAC containing either light (KORO) or heavy (K8R10) lysine and arginine.

The lysosome fraction was enriched as described in 6.2.8 (including whole cell lysate for full proteome analysis). Proteins were separated on a $10 \%$ SDS-PAGE followed by Coomassie protein staining with InstantBlue (Expedeon) and subjected to an in-gel digest.

In-gel digest

Gel lanes were cut into equal pieces and subjected to in-gel digestion. Gel pieces were destained three times for $15 \mathrm{~min}$ at $\mathrm{RT}$ with $40 \% \mathrm{EtOH}$ in $50 \mathrm{mM} \mathrm{ABC}$, and subsequently dehydrated in $100 \% \mathrm{EtOH}$ two times for $5 \mathrm{~min}$ at RT. The supernatant was removed and the gel pieces were dried by SpeedVac vacuum centrifugation. The proteins were reduced with $10 \mathrm{mM}$ DTT for $45 \mathrm{~min}$ at $60^{\circ} \mathrm{C}$ and alkylated with $55 \mathrm{mM}$ CAA for $30 \mathrm{~min}$ at RT in the dark. The gel pieces were again washed and destained with $40 \% \mathrm{EtOH}$ in $50 \mathrm{mM} \mathrm{ABC}$, and subsequently dehydrated in $100 \% \mathrm{EtOH}$. The supernatant was removed and the gel pieces were dried by 
SpeedVac vacuum centrifugation. The samples were rehydrated with digestion buffer containing $5 \mathrm{ng} / \mu \mathrm{l}$ Trypsin and incubated over night at $37^{\circ} \mathrm{C}$. Elution of peptides was performed using an increasing acetonitrile concentration from $30 \%$ to $100 \%$. The supernatants were combined and concentrated by SpeedVac vacuum centrifugation. Samples were acidified with STAGE tip loading buffer.

\section{$\mathrm{C}_{18}$ STAGE tip purification}

Purification and concentration for lysosome proteome and full proteome samples was performed using $\mathrm{C}_{18}$ STAGE tips as described before for the diGly IP (6.2.10.1).

\section{LC-MS/MS analysis}

The analysis by liquid chromatography and mass spectrometry was done by Florian Bonn (Institute of Biochemistry II, Goethe University Frankfurt).

The LC-MS analysis was performed as described above for the proteome samples, but with a shorter gradient of $23 \mathrm{~min}$ for a total run time of $35 \mathrm{~min}$.

Data analysis was done with MaxQuant 1.5.1 against the Uniprot Human Reference Proteome database with standard settings and activated SILAC quantification (K8, R10). Differentially abundant proteins $(p<0.05)$ were detected with a One-sided T-Test with perseus.

\subsubsection{Secretome}

The secretome analysis was done with a SILAC based approach. RPE1 NHT and USP32KO cells were grown in 6 well dishes for two weeks in DMEM suitable for SILAC containing either light (KORO) or heavy (K8R10) lysine and arginine.

Secreted proteins in the supernatant were enriched with StrataClean beads as described in 6.2.9. Cells were lysed for full proteome analysis with $100 \mu \mathrm{l}$ Urea buffer and sonicated. Lysates were cleared by highspeed centrifugation at $13,000 \mathrm{rpm}$ for $15 \mathrm{~min}$ at $4{ }^{\circ} \mathrm{C}$ and transferred to a fresh tube. Both bead samples and whole cell lysates were mixed with gel loading buffer and boiled for $5 \mathrm{~min}$ at $95^{\circ} \mathrm{C}$. Proteins were separated on a $12 \%$ SDS-PAGE followed by Coomassie protein staining with InstantBlue (Expedeon) and subjected to an in-gel digest and LC-MS/MS analysis in collaboration with Florian Bonn (Institute of Biochemistry II, Goethe University Frankfurt) as described for the lysosome proteome in 6.2.10.2

\subsubsection{LAMTOR1 ubiquitination sites and interactome}

The interactome analysis of LAMTOR1 was performed in a label-free manner. LAMTOR1-GFP was immunoprecipitated from transfected RPE1 cells using GFP-Trap Agarose beads as described in 6.2.5.1.

\section{On-bead in-solution digest and SDB-RPS STAGE tip purification}

The samples were washed six times with dilution buffer to remove all detergents. After the final IP wash, the SDC (sodium deoxycholate) elution buffer was added directly to the beads and samples were boiled for $10 \mathrm{~min}$ at $60^{\circ} \mathrm{C}$. The cooled down samples were incubated with $500 \mathrm{ng}$ LysC/trypsin in $50 \mathrm{mM}$ Tris $\mathrm{pH} 8.5$ over night at $37^{\circ} \mathrm{C}$. Digested samples were mixed with $1 \%$ TFA in isopropanol to stop the digestion and directly loaded on in-house assembled SDB-RPS STAGE tips. Following two wash steps with $1 \%$ TFA in isopropanol and $0.2 \%$ TFA in 
water, peptides were eluted with $1.25 \%$ ammoniumhydroxide in $80 \% \mathrm{ACN}$ and dried by SpeedVac vacuum centrifugation for storage at $-20^{\circ} \mathrm{C}$.

\section{LC-MS/MS analysis}

The analysis by liquid chromatography and mass spectrometry was done by Georg Tascher (Institute of Biochemistry II, Goethe University Frankfurt).

Dried peptides were reconstituted in 2\% ACN, 0.1\% TFA and analysed on a Q Exactive HF mass spectrometer coupled to an easy nLC 1200 using a $35 \mathrm{~cm}$ long, $75 \mu \mathrm{m}$ ID fused-silica column packed in house with $1.9 \mu \mathrm{m} \mathrm{C}_{18}$ particles, and kept at $50{ }^{\circ} \mathrm{C}$ using an integrated column oven. Peptides were eluted by a non-linear gradient from 4-28\% acetonitrile over $45 \mathrm{~min}$ and directly sprayed into the mass-spectrometer equipped with a nanoFlex ion source. Full scan MS spectra $(300-1650 \mathrm{~m} / \mathrm{z})$ were acquired in profile mode at a resolution of 60,000 at $\mathrm{m} / \mathrm{z} 200$, a maximum injection time of $20 \mathrm{~ms}$ and an AGC target value of $3 \times 10^{6}$ charges. Up to 15 most intense peptides per full scan were isolated using a 1.4 The window and fragmented using higher energy collisional dissociation (normalised collision energy of 28). MS/MS spectra were acquired in centroid mode with a resolution of 30,000, a maximum injection time of $45 \mathrm{~ms}$ and an AGC target value of $1 \times 10^{5}$. Single charged ions, ions with a charge state above 4 and ions with unassigned charge states were not considered for fragmentation and dynamic exclusion was set to 20 s to minimize the acquisition of fragment spectra of already acquired precursors.

\section{Mass spectrometry data processing}

MS raw data was processed with MaxQuant (v 1.6.14.0) applying default parameters. Acquired spectra were searched against the human "one sequence per gene" database (Taxonomy ID 9606) downloaded from UniProt (2020-03-12; 20531 sequences), and a collection of 244 common contaminants ("contaminants.fasta" provided with MaxQuant) using the Andromeda search engine integrated in MaxQuant (Cox et al., 2011; Tyanova et al., 2016a). Searches were performed using default parameters but adding the GlyGly-remnant on Lysines as a variable modification. Identifications were filtered to obtain false discovery rates (FDR) below $1 \%$ for GlyGly-sites, peptide spectrum matches (PSM; minimum length of 7 amino acids) and proteins using a target-decoy strategy (Elias and Gygi, 2007). Protein quantification and data normalization relied on the MaxLFQ algorithm implemented in MaxQuant (Cox et al., 2014).

The MaxQuant output ("proteinGroups.txt", "GlyGly(K)sites.txt") was processed in Perseus (v. 1.6.7.0, (Tyanova et al., 2016b)). First, proteins only identified by a single modified peptide ("only identified by site") or matching to the reversed or contaminants databases were removed. Only proteins with three valid values in at least one of the groups were kept for statistical analysis following the imputation of missing values from a normal distribution (width: 0.3, down-shift: 1.8). For the analysis of the GlyGly-sites, only sites quantified in at least 2 replicates in each condition (NHT, USP32KO) were considered and missing values were not imputed. Significantly changing proteins and GlyGly-sites were defined by a Student's t-test ( $p$ value $<0.05)$ adding an additional minimum fold-change cut-off $(>1.5)$. 


\section{References}

Abdul Rehman, S.A., Kristariyanto, Y.A., Choi, S.-Y., Nkosi, P.J., Weidlich, S., Labib, K., Hofmann, K., and Kulathu, Y. (2016). MINDY-1 Is a Member of an Evolutionarily Conserved and Structurally Distinct New Family of Deubiquitinating Enzymes. In Mol Cell, pp. 146-155.

Aicart-Ramos, C., Valero, R.A., and Rodriguez-Crespo, I. (2011). Protein palmitoylation and subcellular trafficking. In Biochim Biophys Acta, pp. 2981-2994.

Akhavantabasi, S., Akman, H.B., Sapmaz, A., Keller, J., Petty, E.M., and Erson, A.E. (2010). USP32 is an active, membrane-bound ubiquitin protease overexpressed in breast cancers. In Mamm Genome (Springer-Verlag), pp. 388-397.

Akutsu, M., Dikic, I., and Bremm, A. (2016). Ubiquitin chain diversity at a glance. In J Cell Sci (The Company of Biologists Ltd), pp. 875-880.

Anant, J.S., Desnoyers, L., Machius, M., Demeler, B., Hansen, J.C., Westover, K.D., Deisenhofer, J., and Seabra, M.C. (1998). Mechanism of Rab geranylgeranylation: formation of the catalytic ternary complex. In Biochemistry (American Chemical Society), pp. 12559-12568.

Andres, D.A., Goldstein, J.L., Ho, Y.K., and Brown, M.S. (1993). Mutational analysis of alphasubunit of protein farnesyltransferase. Evidence for a catalytic role. The Journal of biological chemistry 268, 1383-1390.

Aniento, F., Emans, N., Griffiths, G., and Gruenberg, J. (1993). Cytoplasmic dynein-dependent vesicular transport from early to late endosomes. In The Journal of cell biology, pp. 1373-1387.

Ballabio, A., and Bonifacino, J.S. (2020). Lysosomes as dynamic regulators of cell and organismal homeostasis. In Nature Reviews Molecular Cell Biology (Nature Publishing Group), pp. 101-118.

Bar-Peled, L., Chantranupong, L., Cherniack, A.D., Chen, W.W., Ottina, K.A., Grabiner, B.C., Spear, E.D., Carter, S.L., Meyerson, M., and Sabatini, D.M. (2013). A Tumor suppressor complex with GAP activity for the Rag GTPases that signal amino acid sufficiency to mTORC1. In Science (American Association for the Advancement of Science), pp. 1100-1106.

Bar-Peled, L., Schweitzer, L.D., Zoncu, R., and Sabatini, D.M. (2012). Ragulator is a GEF for the rag GTPases that signal amino acid levels to mTORC1. In Cell, pp. 1196-1208.

BasuRay, S., Agola, J.O., Jim, P.A., Seaman, M.N., and Wandinger-Ness, A. (2012). Rab7a in Endocytosis and Signaling. In Encyclopedia of Signaling Molecules (Springer New York), pp. 1536-1547.

Bhuin, T., and Roy, J.K. (2015). Rab11 in disease progression. In Int J Mol Cell Med (Babol University of Medical Sciences), pp. 1-8.

Bissig, C., and Gruenberg, J. (2014). ALIX and the multivesicular endosome: ALIX in Wonderland. In Trends in Cell Biology, pp. 19-25.

Blanc, M., David, F., Abrami, L., Migliozzi, D., Armand, F., Bürgi, J., and van der Goot, F.G. (2015). SwissPalm: Protein Palmitoylation database. In F1000Res (F1000 Research Limited), pp. 261.

Blount, J.R., Johnson, S.L., and Todi, S.V. (2020). Unanchored Ubiquitin Chains, Revisited. In Front Cell Dev Biol (Frontiers), pp. 582361.

Bongaarts, A., van Scheppingen, J., Korotkov, A., Mijnsbergen, C., Anink, J.J., Jansen, F.E., Spliet, W.G.M., den Dunnen, W.F.A., Gruber, V.E., Scholl, T., et al. (2020). The coding and noncoding transcriptional landscape of subependymal giant cell astrocytomas. In Brain, pp. 131149.

Bonifacino, J.S. (2004). The GGA proteins: adaptors on the move. In Nature Reviews Molecular Cell Biology (Nature Publishing Group), pp. 23-32.

Bonifacino, J.S., and Hurley, J.H. (2008). Retromer. In Current Opinion in Cell Biology, pp. 427436. 
Braulke, T., and Bonifacino, J.S. (2009). Sorting of lysosomal proteins. In Biochim Biophys Acta, pp. 605-614.

Bucci, C., Parton, R.G., Mather, I.H., Stunnenberg, H., Simons, K., Hoflack, B., and Zerial, M. (1992). The small GTPase rab5 functions as a regulatory factor in the early endocytic pathway. In Cell, pp. 715-728.

Campa, C.C., Margaria, J.P., Derle, A., Del Giudice, M., De Santis, M.C., Gozzelino, L., Copperi, F., Bosia, C., and Hirsch, E. (2018). Rab11 activity and PtdIns(3)P turnover removes recycling cargo from endosomes. In Nat Chem Biol (Nature Publishing Group), pp. 801-810.

Carpenter, A.E., Jones, T.R., Lamprecht, M.R., Clarke, C., Kang, I.H., Friman, O., Guertin, D.A., Chang, J.H., Lindquist, R.A., Moffat, J., et al. (2006). CellProfiler: image analysis software for identifying and quantifying cell phenotypes. In Genome Biol (BioMed Central), pp. R100.

Casey, P.J., and Seabra, M.C. (1996). Protein prenyltransferases. In J Biol Chem, pp. 5289-5292.

Chen, Y.-C., Navarrete, M.S., Wang, Y., McClintock, N.C., Sakurai, R., Wang, F., Chen, K.T., Chou, T.-F., Rehan, V.K., Lee, D.J., et al. (2020). N-myristoyltransferase-1 is necessary for lysosomal degradation and mTORC1 activation in cancer cells. In Sci Rep (Nature Publishing Group), pp. $11952-11918$.

Chung, C.Y.-S., Shin, H.R., Berdan, C.A., Ford, B., Ward, C.C., Olzmann, J.A., Zoncu, R., and Nomura, D.K. (2019). Covalent targeting of the vacuolar H+-ATPase activates autophagy via mTORC1 inhibition. In Nat Chem Biol (Nature Publishing Group), pp. 776-785.

Clague, M.J., Barsukov, I., Coulson, J.M., Liu, H., Rigden, D.J., and Urbé, S. (2013). Deubiquitylases from genes to organism. In Physiol Rev (American Physiological Society Bethesda, MD), pp. 1289-1315.

Clague, M.J., Heride, C., and Urbé, S. (2015). The demographics of the ubiquitin system. In Trends in Cell Biology, pp. 417-426.

Clague, M.J., Liu, H., and Urbé, S. (2012). Governance of endocytic trafficking and signaling by reversible ubiquitylation. In Dev Cell, pp. 457-467.

Clague, M.J., and Urbé, S. (2017). Integration of cellular ubiquitin and membrane traffic systems: focus on deubiquitylases. In The FEBS Journal.

Clague, M.J., Urbé, S., and Komander, D. (2019). Breaking the chains: deubiquitylating enzyme specificity begets function. In Nature Reviews Molecular Cell Biology (Nature Publishing Group), pp. 338-352.

Collinet, C., Stöter, M., Bradshaw, C.R., Samusik, N., Rink, J.C., Kenski, D., Habermann, B., Buchholz, F., Henschel, R., Mueller, M.S., et al. (2010). Systems survey of endocytosis by multiparametric image analysis. In Nature (Nature Publishing Group), pp. 243-249.

Cox, J., Hein, M.Y., Luber, C.A., Paron, I., Nagaraj, N., and Mann, M. (2014). Accurate proteomewide label-free quantification by delayed normalization and maximal peptide ratio extraction, termed MaxLFQ. In Mol Cell Proteomics, pp. 2513-2526.

Cox, J., and Mann, M. (2008). MaxQuant enables high peptide identification rates, individualized p.p.b.-range mass accuracies and proteome-wide protein quantification. Nature Biotechnology 26, 1367-1372.

Cox, J., Neuhauser, N., Michalski, A., Scheltema, R.A., Olsen, J.V., and Mann, M. (2011). Andromeda: a peptide search engine integrated into the MaxQuant environment. In J Proteome Res (American Chemical Society), pp. 1794-1805.

de Araujo, M.E.G., Naschberger, A., Fürnrohr, B.G., Stasyk, T., Dunzendorfer-Matt, T., Lechner, S., Welti, S., Kremser, L., Shivalingaiah, G., Offterdinger, M., et al. (2017). Crystal structure of the human lysosomal mTORC1 scaffold complex and its impact on signaling. In Science (American Association for the Advancement of Science), pp. 377-381.

de Duve, C. (2005). The lysosome turns fifty. In Nat Cell Biol (Nature Publishing Group), pp. 847849.

de Jong, R.N., Ab, E., Diercks, T., Truffault, V., Daniëls, M., Kaptein, R., and Folkers, G.E. (2006). Solution structure of the human ubiquitin-specific protease 15 DUSP domain. In J Biol Chem, pp. 5026-5031. 
Del Conte-Zerial, P., Brusch, L., Rink, J.C., Collinet, C., Kalaidzidis, Y., Zerial, M., and Deutsch, A. (2008). Membrane identity and GTPase cascades regulated by toggle and cut-out switches. In Mol Syst Biol (John Wiley \& Sons, Ltd), pp. 206.

Di Malta, C., Siciliano, D., Calcagni, A., Monfregola, J., Punzi, S., Pastore, N., Eastes, A.N., Davis, O., De Cegli, R., Zampelli, A., et al. (2017). Transcriptional activation of RagD GTPase controls mTORC1 and promotes cancer growth. In Science (American Association for the Advancement of Science), pp. 1188-1192.

Doench, J.G., Fusi, N., Sullender, M., Hegde, M., Vaimberg, E.W., Donovan, K.F., Smith, I., Tothova, Z., Wilen, C., Orchard, R., et al. (2016). Optimized sgRNA design to maximize activity and minimize off-target effects of CRISPR-Cas9. In Nat Biotechnol (Nature Publishing Group), pp. 184-191.

Dossou, A.S., and Basu, A. (2019). The Emerging Roles of mTORC1 in Macromanaging Autophagy. In Cancers (Basel) (Multidisciplinary Digital Publishing Institute), pp. 1422.

Dou, N., Hu, Q., Li, L., Wu, Q., Li, Y., and Gao, Y. (2020). USP32 promotes tumorigenesis and chemoresistance in gastric carcinoma via upregulation of SMAD2. In Int J Biol Sci, pp. 16481657.

Elias, J.E., and Gygi, S.P. (2007). Target-decoy search strategy for increased confidence in largescale protein identifications by mass spectrometry. In Nat Methods (Nature Publishing Group), pp. 207-214.

Elkin, S.R., Lakoduk, A.M., and Schmid, S.L. (2016). Endocytic pathways and endosomal trafficking: a primer. In Wien Med Wochenschr (Springer Vienna), pp. 196-204.

Faesen, A.C., Luna-Vargas, M.P.A., Geurink, P.P., Clerici, M., Merkx, R., van Dijk, W.J., Hameed, D.S., El Oualid, F., Ovaa, H., and Sixma, T.K. (2011). The differential modulation of USP activity by internal regulatory domains, interactors and eight ubiquitin chain types. In Chem Biol, pp. 15501561.

Farazi, T.A., Waksman, G., and Gordon, J.I. (2001). The biology and enzymology of protein Nmyristoylation. In J Biol Chem, pp. 39501-39504.

Filipek, P.A., de Araujo, M.E.G., Vogel, G.F., De Smet, C.H., Eberharter, D., Rebsamen, M., Rudashevskaya, E.L., Kremser, L., Yordanov, T., Tschaikner, P., et al. (2017). LAMTOR/Ragulator is a negative regulator of Arl8b-and BORC-dependent late endosomal positioning. The Journal of cell biology 216, 4199-4215.

Follett, J., Norwood, S.J., Hamilton, N.A., Mohan, M., Kovtun, O., Tay, S., Zhe, Y., Wood, S.A., Mellick, G.D., Silburn, P.A., et al. (2014). The Vps35 D620N mutation linked to Parkinson\&apos;s disease disrupts the cargo sorting function of retromer. In Traffic (John Wiley \& Sons, Ltd), pp. 230-244.

Francavilla, C., Papetti, M., Rigbolt, K.T.G., Pedersen, A.-K., Sigurdsson, J.O., Cazzamali, G., Karemore, G., Blagoev, B., and Olsen, J.V. (2016). Multilayered proteomics reveals molecular switches dictating ligand-dependent EGFR trafficking. In Nat Struct Mol Biol (Nature Publishing Group), pp. 608-618.

Fromm, S.A., Lawrence, R.E., and Hurley, J.H. (2020). Structural mechanism for amino aciddependent Rag GTPase nucleotide state switching by SLC38A9. In Nat Struct Mol Biol (Nature Publishing Group), pp. 1017-1023.

Fueller, J., Herbst, K., Meurer, M., Gubicza, K., Kurtulmus, B., Knopf, J.D., Kirrmaier, D., Buchmuller, B.C., Pereira, G., Lemberg, M.K., et al. (2020). CRISPR-Cas12a-assisted PCR tagging of mammalian genes. In The Journal of cell biology.

Funakoshi, Y., Chou, M.M., Kanaho, Y., and Donaldson, J.G. (2014). TRE17/USP6 regulates ubiquitylation and trafficking of cargo proteins that enter cells by clathrin-independent endocytosis. In J Cell Sci (The Company of Biologists Ltd), pp. 4750-4761.

Gao, J., Liao, J., and Yang, G.-Y. (2009). CAAX-box protein, prenylation process and carcinogenesis. In Am J Transl Res (e-Century Publishing Corporation), pp. 312-325. 
Ginsberg, S.D., Mufson, E.J., Alldred, M.J., Counts, S.E., Wuu, J., Nixon, R.A., and Che, S. (2011). Upregulation of select rab GTPases in cholinergic basal forebrain neurons in mild cognitive impairment and Alzheimergapos;s disease. In J Chem Neuroanat, pp. 102-110.

Glick, D., Barth, S., and Macleod, K.F. (2010). Autophagy: cellular and molecular mechanisms. In J Pathol (John Wiley \& Sons, Ltd), pp. 3-12.

Goh, L.K., Huang, F., Kim, W., Gygi, S., and Sorkin, A. (2010). Multiple mechanisms collectively regulate clathrin-mediated endocytosis of the epidermal growth factor receptor. In The Journal of cell biology, pp. 871-883.

Grabbe, C., Husnjak, K., and Dikic, I. (2011). The spatial and temporal organization of ubiquitin networks. In Nature Reviews Molecular Cell Biology (Nature Publishing Group), pp. 295-307.

Gromov, P., and Celis, J.E. (1998). Rab11a is modified in vivo by isoprenoid geranylgeranyl. In Electrophoresis (John Wiley \& Sons, Ltd), pp. 1803-1807.

Gruenberg, J. (2001). The endocytic pathway: a mosaic of domains. In Nature Reviews Molecular Cell Biology (Nature Publishing Group), pp. 721-730.

Guichard, A., Nizet, V., and Bier, E. (2014). RAB11-mediated trafficking in host-pathogen interactions. In Nat Rev Microbiol (Nature Publishing Group), pp. 624-634.

Gutierrez, M.G., Munafó, D.B., Berón, W., and Colombo, M.I. (2004). Rab7 is required for the normal progression of the autophagic pathway in mammalian cells. In J Cell Sci (The Company of Biologists Ltd), pp. 2687-2697.

Hao, Y.-H., Doyle, J.M., Ramanathan, S., Gomez, T.S., Jia, D., Xu, M., Chen, Z.J., Billadeau, D.D., Rosen, M.K., and Potts, P.R. (2013). Regulation of WASH-dependent actin polymerization and protein trafficking by ubiquitination. In Cell, pp. 1051-1064.

Hao, Y.-H., Fountain, M.D., Fon Tacer, K., Xia, F., Bi, W., Kang, S.-H.L., Patel, A., Rosenfeld, J.A., Le Caignec, C., Isidor, B., et al. (2015). USP7 Acts as a Molecular Rheostat to Promote WASHDependent Endosomal Protein Recycling and Is Mutated in a Human Neurodevelopmental Disorder. In Mol Cell, pp. 956-969.

Henne, W.M., Buchkovich, N.J., and Emr, S.D. (2011). The ESCRT pathway. In Dev Cell, pp. 7791.

Hershko, A., and Ciechanover, A. (1998). The ubiquitin system. In Annu Rev Biochem ( Annual Reviews 4139 El Camino Way, P.O. Box 10139, Palo Alto, CA 94303-0139, USA), pp. 425-479.

Hjerpe, R., Aillet, F., Lopitz-Otsoa, F., Lang, V., England, P., and Rodriguez, M.S. (2009). Efficient protection and isolation of ubiquitylated proteins using tandem ubiquitin-binding entities. EMBO reports 10, 1250-1258.

Hornbeck, P.V., Zhang, B., Murray, B., Kornhauser, J.M., Latham, V., and Skrzypek, E. (2015). PhosphoSitePlus, 2014: mutations, PTMs and recalibrations. In Nucleic Acids Res, pp. D512520.

Hu, W., Wei, H., Li, K., Li, P., Lin, J., and Feng, R. (2017). Downregulation of USP32 inhibits cell proliferation, migration and invasion in human small cell lung cancer. In Cell Prolif, pp. e12343.

Hu, Y.-B., Dammer, E.B., Ren, R.-J., and Wang, G. (2015). The endosomal-lysosomal system: from acidification and cargo sorting to neurodegeneration. In Transl Neurodegener (BioMed Central), pp. 18-10.

Huang, F., Kirkpatrick, D., Jiang, X., Gygi, S., and Sorkin, A. (2006). Differential regulation of EGF receptor internalization and degradation by multiubiquitination within the kinase domain. In Mol Cell, pp. 737-748.

Huotari, J., and Helenius, A. (2011). Endosome maturation. In EMBO J (John Wiley \& Sons, Ltd), pp. 3481-3500.

Hutagalung, A.H., and Novick, P.J. (2011). Role of Rab GTPases in membrane traffic and cell physiology. In Physiol Rev (American Physiological Society Bethesda, MD), pp. 119-149.

Huynh, K.K., and Grinstein, S. (2007). Regulation of vacuolar pH and its modulation by some microbial species. In Microbiol Mol Biol Rev (American Society for Microbiology), pp. 452-462. 
Inami, Y., Yamashina, S., Izumi, K., Ueno, T., Tanida, I., Ikejima, K., and Watanabe, S. (2011). Hepatic steatosis inhibits autophagic proteolysis via impairment of autophagosomal acidification and cathepsin expression. In Biochem Biophys Res Commun, pp. 618-625.

Jäger, S., Bucci, C., Tanida, I., Ueno, T., Kominami, E., Saftig, P., and Eskelinen, E.-L. (2004). Role for Rab7 in maturation of late autophagic vacuoles. In $\mathrm{J}$ Cell Sci (The Company of Biologists Ltd), pp. 4837-4848.

Jewell, J.L., Russell, R.C., and Guan, K.-L. (2013). Amino acid signalling upstream of mTOR. In Nature Reviews Molecular Cell Biology (Nature Publishing Group), pp. 133-139.

Jia, J., Abudu, Y.P., Claude-Taupin, A., Gu, Y., Kumar, S., Choi, S.W., Peters, R., Mudd, M.H., Allers, L., Salemi, M., et al. (2018). Galectins Control mTOR in Response to Endomembrane Damage. In Mol Cell, pp. 120-135.e128.

Jiang, H., Zhang, X., Chen, X., Aramsangtienchai, P., Tong, Z., and Lin, H. (2018). Protein Lipidation: Occurrence, Mechanisms, Biological Functions, and Enabling Technologies. In Chem Rev (American Chemical Society), pp. 919-988.

Jordens, I., Fernandez-Borja, M., Marsman, M., Dusseljee, S., Janssen, L., Calafat, J., Janssen, H., Wubbolts, R., and Neefjes, J. (2001). The Rab7 effector protein RILP controls lysosomal transport by inducing the recruitment of dynein-dynactin motors. In Curr Biol, pp. 1680-1685.

Jovic, M., Sharma, M., Rahajeng, J., and Caplan, S. (2010). The early endosome: a busy sorting station for proteins at the crossroads. In Histol Histopathol, pp. 99-112.

Jung, J., Genau, H.M., and Behrends, C. (2015). Amino Acid-Dependent mTORC1 Regulation by the Lysosomal Membrane Protein SLC38A9. In Mol Cell Biol (American Society for Microbiology), pp. 2479-2494.

Kaizuka, T., Morishita, H., Hama, Y., Tsukamoto, S., Matsui, T., Toyota, Y., Kodama, A., Ishihara, T., Mizushima, T., and Mizushima, N. (2016). An Autophagic Flux Probe that Releases an Internal Control. In Mol Cell, pp. 835-849.

Kanemitsu-Fujita, A., Morishita, S., Kjaer, S., Fukuda, M., Schiavo, G., and Nakamura, T. (2018). Comparable affinity of RabGDl $\alpha$ to GTP- and GDP-bound forms of Rab7 supports a four-state transition model for Rab7 subcellular localization. In bioRxiv, pp. 287516.

Kim, E., Goraksha-Hicks, P., Li, L., Neufeld, T.P., and Guan, K.-L. (2008). Regulation of TORC1 by Rag GTPases in nutrient response. In Nat Cell Biol (Nature Publishing Group), pp. 935-945.

Kim, Y.-M., Jung, C.H., Seo, M., Kim, E.K., Park, J.-M., Bae, S.S., and Kim, D.-H. (2015). mTORC1 phosphorylates UVRAG to negatively regulate autophagosome and endosome maturation. In Mol Cell, pp. 207-218.

Kirisako, T., Kamei, K., Murata, S., Kato, M., Fukumoto, H., Kanie, M., Sano, S., Tokunaga, F., Tanaka, K., and Iwai, K. (2006). A ubiquitin ligase complex assembles linear polyubiquitin chains. In EMBO J (John Wiley \& Sons, Ltd), pp. 4877-4887.

Kobayashi, T., Beuchat, M.-H., Chevallier, J., Makino, A., Mayran, N., Escola, J.-M., Lebrand, C., Cosson, P., Kobayashi, T., and Gruenberg, J. (2002). Separation and characterization of late endosomal membrane domains. In J Biol Chem, pp. 32157-32164.

Koerver, L., Papadopoulos, C., Liu, B., Kravic, B., Rota, G., Brecht, L., Veenendaal, T., Polajnar, M., Bluemke, A., Ehrmann, M., et al. (2019). The ubiquitin-conjugating enzyme UBE2QL1 coordinates lysophagy in response to endolysosomal damage. In EMBO reports (John Wiley \& Sons, Ltd), pp. e48014.

Komander, D., Clague, M.J., and Urbé, S. (2009). Breaking the chains: structure and function of the deubiquitinases. In Nature Reviews Molecular Cell Biology (Nature Publishing Group), pp. 550-563.

Korolchuk, V.I, Saiki, S., Lichtenberg, M., Siddiqi, F.H., Roberts, E.A., Imarisio, S., Jahreiss, L., Sarkar, S., Futter, M., Menzies, F.M., et al. (2011). Lysosomal positioning coordinates cellular nutrient responses. In Nat Cell Biol (Nature Publishing Group), pp. 453-460.

Kulak, N.A., Pichler, G., Paron, I., Nagaraj, N., and Mann, M. (2014). Minimal, encapsulated proteomic-sample processing applied to copy-number estimation in eukaryotic cells. In Nat Methods (Nature Publishing Group), pp. 319-324. 
Kwasna, D., Abdul Rehman, S.A., Natarajan, J., Matthews, S., Madden, R., De Cesare, V., Weidlich, S., Virdee, S., Ahel, I., Gibbs-Seymour, I., et al. (2018). Discovery and Characterization of ZUFSP/ZUP1, a Distinct Deubiquitinase Class Important for Genome Stability. In Mol Cell, pp. 150-164.e156.

Lawrence, R.E., Cho, K.F., Rappold, R., Thrun, A., Tofaute, M., Kim, D.J., Moldavski, O., Hurley, J.H., and Zoncu, R. (2018). A nutrient-induced affinity switch controls mTORC1 activation by its Rag GTPase-Ragulator lysosomal scaffold. In Nat Cell Biol (Nature Publishing Group), pp. 10521063.

Levkowitz, G., Waterman, H., Ettenberg, S.A., Katz, M., Tsygankov, A.Y., Alroy, I., Lavi, S., Iwai, K., Reiss, Y., Ciechanover, A., et al. (1999). Ubiquitin ligase activity and tyrosine phosphorylation underlie suppression of growth factor signaling by c-Cbl/Sli-1. In Mol Cell, pp. 1029-1040.

Lewit-Bentley, A., and Réty, S. (2000). EF-hand calcium-binding proteins. In Current Opinion in Structural Biology, pp. 637-643.

Li, C., Shah, S.Z.A., Zhao, D., and Yang, L. (2016). Role of the Retromer Complex in Neurodegenerative Diseases. In Front Aging Neurosci (Frontiers), pp. 42.

Li, P., Zhang, L., Li, Z., Xu, C., Du, X., and Wu, S. (2020). Cas12a mediates efficient and precise endogenous gene tagging via MITI: microhomology-dependent targeted integrations. In Cell Mol Life Sci (Springer International Publishing), pp. 3875-3884.

Lin, X., Zhang, J., Chen, L., Chen, Y., Xu, X., Hong, W., and Wang, T. (2017). Tyrosine phosphorylation of Rab7 by Src kinase. In Cell Signal, pp. 84-94.

Liu, Z., Lv, Y.J., Song, Y.P., Li, X.H., Du, Y.N., Wang, C.H., and Hu, L.K. (2012). Lysosomal membrane protein TMEM192 deficiency triggers crosstalk between autophagy and apoptosis in HepG2 hepatoma cells. In Oncol Rep (Spandidos Publications), pp. 985-991.

Long, X., Lin, Y., Ortiz-Vega, S., Yonezawa, K., and Avruch, J. (2005). Rheb binds and regulates the mTOR kinase. In Curr Biol, pp. 702-713.

Longatti, A., Lamb, C.A., Razi, M., Yoshimura, S.-i., Barr, F.A., and Tooze, S.A. (2012). TBC1D14 regulates autophagosome formation via Rab11- and ULK1-positive recycling endosomes. In The Journal of cell biology, pp. 659-675.

Luzio, J.P., Pryor, P.R., and Bright, N.A. (2007). Lysosomes: fusion and function. In Nature Reviews Molecular Cell Biology (Nature Publishing Group), pp. 622-632.

MacDonald, E., Urbé, S., and Clague, M.J. (2014). USP8 controls the trafficking and sorting of lysosomal enzymes. In Traffic (John Wiley \& Sons A/S), pp. 879-888.

Madan, B., Walker, M.P., Young, R., Quick, L., Orgel, K.A., Ryan, M., Gupta, P., Henrich, I.C., Ferrer, M., Marine, S., et al. (2016). USP6 oncogene promotes Wnt signaling by deubiquitylating Frizzleds. In Proc Natl Acad Sci USA (National Academy of Sciences), pp. E2945-2954.

Maday, S., Wallace, K.E., and Holzbaur, E.L.F. (2012). Autophagosomes initiate distally and mature during transport toward the cell soma in primary neurons. In The Journal of cell biology, pp. 407-417.

Magraoui, F.E., Reidick, C., Meyer, H.E., and Platta, H.W. (2015). Autophagy-Related Deubiquitinating Enzymes Involved in Health and Disease. Cells 4, 596-621.

Mari, M., Bujny, M.V., Zeuschner, D., Geerts, W.J.C., Griffith, J., Petersen, C.M., Cullen, P.J., Klumperman, J., and Geuze, H.J. (2008). SNX1 defines an early endosomal recycling exit for sortilin and mannose 6-phosphate receptors. In Traffic (John Wiley \& Sons, Ltd), pp. 380-393.

Martinu, L., Masuda-Robens, J.M., Robertson, S.E., Santy, L.C., Casanova, J.E., and Chou, M.M. (2004). The TBC (Tre-2/Bub2/Cdc16) domain protein TRE17 regulates plasma membraneendosomal trafficking through activation of Arf6. In Mol Cell Biol (American Society for Microbiology Journals), pp. 9752-9762.

Mattern, M., Sutherland, J., Kadimisetty, K., Barrio, R., and Rodriguez, M.S. (2019). Using Ubiquitin Binders to Decipher the Ubiquitin Code. Trends in biochemical sciences 44, 599-615.

Maxfield, F.R., and Yamashiro, D.J. (1987). Endosome acidification and the pathways of receptor-mediated endocytosis. In Adv Exp Med Biol (Springer, Boston, MA), pp. 189-198. 
McCray, B.A., Skordalakes, E., and Taylor, J.P. (2010). Disease mutations in Rab7 result in unregulated nucleotide exchange and inappropriate activation. In Hum Mol Genet, pp. 10331047.

McCullough, J., Clague, M.J., and Urbé, S. (2004). AMSH is an endosome-associated ubiquitin isopeptidase. In The Journal of cell biology, pp. 487-492.

McMillan, K.J., Korswagen, H.C., and Cullen, P.J. (2017). The emerging role of retromer in neuroprotection. In Current Opinion in Cell Biology, pp. 72-82.

Medina, D.L., Di Paola, S., Peluso, I., Armani, A., De Stefani, D., Venditti, R., Montefusco, S., ScottoRosato, A., Prezioso, C., Forrester, A., et al. (2015). Lysosomal calcium signalling regulates autophagy through calcineurin and TFEB. In Nat Cell Biol (Nature Research), pp. 288-299.

Mellman, I. (1996). Endocytosis and molecular sorting. In Annu Rev Cell Dev Biol ( Annual Reviews 4139 El Camino Way, P.O. Box 10139, Palo Alto, CA 94303-0139, USA), pp. 575-625.

Méresse, S., Gorvel, J.P., and Chavrier, P. (1995). The rab7 GTPase resides on a vesicular compartment connected to lysosomes. In J Cell Sci (J Cell Sci), pp. 3349-3358.

Mertins, P., Qiao, J.W., Patel, J., Udeshi, N.D., Clauser, K.R., Mani, D.R., Burgess, M.W., Gillette, M.A., Jaffe, J.D., and Carr, S.A. (2013). Integrated proteomic analysis of post-translational modifications by serial enrichment. In Nat Methods (Nature Publishing Group), pp. 634-637.

Mevissen, T.E.T., Hospenthal, M.K., Geurink, P.P., Elliott, P.R., Akutsu, M., Arnaudo, N., Ekkebus, R., Kulathu, Y., Wauer, T., El Oualid, F., et al. (2013). OTU deubiquitinases reveal mechanisms of linkage specificity and enable ubiquitin chain restriction analysis. In Cell, pp. 169-184.

Mizushima, N. (2007). Autophagy: process and function. In Genes Dev (Cold Spring Harbor Lab), pp. 2861-2873.

Modica, G., and Lefrancois, S. (2020). Post-translational modifications: How to modulate Rab7 functions. In Small GTPases (Taylor \& Francis), pp. 167-173.

Modica, G., Skorobogata, O., Sauvageau, E., Vissa, A., Yip, C.M., Kim, P.K., Wurtele, H., and Lefrancois, S. (2017). Rab7 palmitoylation is required for efficient endosome-to-TGN trafficking. In J Cell Sci (The Company of Biologists Ltd), pp. 2579-2590.

Morrow, M.E., Morgan, M.T., Clerici, M., Growkova, K., Yan, M., Komander, D., Sixma, T.K., Simicek, M., and Wolberger, C. (2018). Active site alanine mutations convert deubiquitinases into high-affinity ubiquitin-binding proteins. EMBO reports 19.

Müller, M.P., and Goody, R.S. (2018). Molecular control of Rab activity by GEFs, GAPs and GDI. In Small GTPases (Taylor \& Francis), pp. 5-21.

Mullock, B.M., Bright, N.A., Fearon, C.W., Gray, S.R., and Luzio, J.P. (1998). Fusion of lysosomes with late endosomes produces a hybrid organelle of intermediate density and is NSF dependent. In The Journal of cell biology, pp. 591-601.

Murray, J.T., Panaretou, C., Stenmark, H., Miaczynska, M., and Backer, J.M. (2002). Role of Rab5 in the recruitment of hVps34/p150 to the early endosome. In Traffic (John Wiley \& Sons, Ltd), pp. 416-427.

Nada, S., Hondo, A., Kasai, A., Koike, M., Saito, K., Uchiyama, Y., and Okada, M. (2009). The novel lipid raft adaptor p18 controls endosome dynamics by anchoring the MEK-ERK pathway to late endosomes. In EMBO J (John Wiley \& Sons, Ltd), pp. 477-489.

Nazio, F., Strappazzon, F., Antonioli, M., Bielli, P., Cianfanelli, V., Bordi, M., Gretzmeier, C., Dengjel, J., Piacentini, M., Fimia, G.M., et al. (2013). mTOR inhibits autophagy by controlling ULK1 ubiquitylation, self-association and function through AMBRA1 and TRAF6. In Nat Cell Biol (Nature Publishing Group), pp. 406-416.

Nguyen, T.L., Schneppenheim, J., Rudnik, S., Lüllmann-Rauch, R., Bernreuther, C., HermansBorgmeyer, I., Glatzel, M., Saftig, P., and Schröder, B. (2017). Functional characterization of the lysosomal membrane protein TMEM192 in mice. In Oncotarget (Impact Journals), pp. 4363543652.

Nielsen, E., Severin, F., Backer, J.M., Hyman, A.A., and Zerial, M. (1999). Rab5 regulates motility of early endosomes on microtubules. In Nat Cell Biol (Nature Publishing Group), pp. 376-382. 
Pankiv, S., Alemu, E.A., Brech, A., Bruun, J.-A., Lamark, T., Overvatn, A., Bjørkøy, G., and Johansen, T. (2010). FYCO1 is a Rab7 effector that binds to LC3 and PI3P to mediate microtubule plus end-directed vesicle transport. In The Journal of cell biology, pp. 253-269.

Papadopoulos, C., Kirchner, P., Bug, M., Grum, D., Koerver, L., Schulze, N., Poehler, R., Dressler, A., Fengler, S., Arhzaouy, K., et al. (2017). VCP/p97 cooperates with YOD1, UBXD1 and PLAA to drive clearance of ruptured lysosomes by autophagy. In EMBO J, pp. 135-150.

Paulding, C.A., Ruvolo, M., and Haber, D.A. (2003). The Tre2 (USP6) oncogene is a hominoidspecific gene. In Proceedings of the National Academy of Sciences (National Academy of Sciences), pp. 2507-2511.

Pereira-Leal, J.B., Hume, A.N., and Seabra, M.C. (2001). Prenylation of Rab GTPases: molecular mechanisms and involvement in genetic disease. In FEBS Letters (John Wiley \& Sons, Ltd), pp. 197-200.

Pickart, C.M., and Rose, I.A. (1985). Ubiquitin carboxyl-terminal hydrolase acts on ubiquitin carboxyl-terminal amides. In J Biol Chem (J Biol Chem), pp. 7903-7910.

Piper, R.C., and Luzio, J.P. (2001). Late endosomes: sorting and partitioning in multivesicular bodies. In Traffic (John Wiley \& Sons, Ltd), pp. 612-621.

Poteryaev, D., Datta, S., Ackema, K., Zerial, M., and Spang, A. (2010). Identification of the switch in early-to-late endosome transition. In Cell, pp. 497-508.

Pu, J., Guardia, C.M., Keren-Kaplan, T., and Bonifacino, J.S. (2016). Mechanisms and functions of lysosome positioning. In J Cell Sci (The Company of Biologists Ltd), pp. 4329-4339.

$\mathrm{Pu}$, J., Keren-Kaplan, T., and Bonifacino, J.S. (2017). A Ragulator-BORC interaction controls lysosome positioning in response to amino acid availability. In The Journal of cell biology, pp. 4183-4197.

Rahighi, S., and Dikic, I. (2012). Selectivity of the ubiquitin-binding modules. In FEBS Letters (John Wiley \& Sons, Ltd), pp. 2705-2710.

Ravikumar, B., Moreau, K., Jahreiss, L., Puri, C., and Rubinsztein, D.C. (2010). Plasma membrane contributes to the formation of pre-autophagosomal structures. In Nat Cell Biol (Nature Publishing Group), pp. 747-757.

Rebsamen, M., Pochini, L., Stasyk, T., de Araujo, M.E.G., Galluccio, M., Kandasamy, R.K., Snijder, B., Fauster, A., Rudashevskaya, E.L., Bruckner, M., et al. (2015). SLC38A9 is a component of the lysosomal amino acid sensing machinery that controls mTORC1. In Nature (Nature Publishing Group), pp. 477-481.

Rink, J., Ghigo, E., Kalaidzidis, Y., and Zerial, M. (2005). Rab conversion as a mechanism of progression from early to late endosomes. In Cell, pp. 735-749.

Rojas, R., van Vlijmen, T., Mardones, G.A., Prabhu, Y., Rojas, A.L., Mohammed, S., Heck, A.J.R., Raposo, G., van der Sluijs, P., and Bonifacino, J.S. (2008). Regulation of retromer recruitment to endosomes by sequential action of Rab5 and Rab7. In The Journal of cell biology, pp. 513-526.

Row, P.E., Prior, I.A., McCullough, J., Clague, M.J., and Urbé, S. (2006). The ubiquitin isopeptidase UBPY regulates endosomal ubiquitin dynamics and is essential for receptor downregulation. In J Biol Chem, pp. 12618-12624.

Saftig, P., and Klumperman, J. (2009). Lysosome biogenesis and lysosomal membrane proteins: trafficking meets function. In Nature Reviews Molecular Cell Biology (Nature Publishing Group), pp. 623-635.

Sahtoe, D.D., and Sixma, T.K. (2015). Layers of DUB regulation. In Trends Biochem Sci, pp. 456 467.

Sancak, Y., Bar-Peled, L., Zoncu, R., Markhard, A.L., Nada, S., and Sabatini, D.M. (2010). Ragulator-Rag complex targets mTORC1 to the lysosomal surface and is necessary for its activation by amino acids. In Cell, pp. 290-303.

Sancak, Y., Peterson, T.R., Shaul, Y.D., Lindquist, R.A., Thoreen, C.C., Bar-Peled, L., and Sabatini, D.M. (2008). The Rag GTPases bind raptor and mediate amino acid signaling to mTORC1. In Science (American Association for the Advancement of Science), pp. 1496-1501. 
Sanders, S.S., De Simone, F.I., and Thomas, G.M. (2019). mTORC1 Signaling Is PalmitoylationDependent in Hippocampal Neurons and Non-neuronal Cells and Involves Dynamic Palmitoylation of LAMTOR1 and mTOR. In Front Cell Neurosci (Frontiers), pp. 115.

Sanders, S.S., Martin, D.D.O., Butland, S.L., Lavallée-Adam, M., Calzolari, D., Kay, C., Yates, J.R., and Hayden, M.R. (2015). Curation of the Mammalian Palmitoylome Indicates a Pivotal Role for Palmitoylation in Diseases and Disorders of the Nervous System and Cancers. In PLoS Comput Biol (Public Library of Science), pp. e1004405.

Sapmaz, A., Berlin, I., Bos, E., Wijdeven, R.H., Janssen, H., Konietzny, R., Akkermans, J.J., ErsonBensan, A.E., Koning, R.I., Kessler, B.M., et al. (2019). USP32 regulates late endosomal transport and recycling through deubiquitylation of Rab7. In Nat Commun (Nature Publishing Group), pp. 1454-1418.

Sardiello, M., Palmieri, M., di Ronza, A., Medina, D.L., Valenza, M., Gennarino, V.A., Di Malta, C., Donaudy, F., Embrione, V., Polishchuk, R.S., et al. (2009). A gene network regulating lysosomal biogenesis and function. In Science (American Association for the Advancement of Science), pp. 473-477.

Savio, M.G., Wollscheid, N., Cavallaro, E., Algisi, V., Di Fiore, P.P., Sigismund, S., Maspero, E., and Polo, S. (2016). USP9X Controls EGFR Fate by Deubiquitinating the Endocytic Adaptor Eps15. In Curr Biol, pp. 173-183.

Saxton, R.A., and Sabatini, D.M. (2017). mTOR Signaling in Growth, Metabolism, and Disease. In Cell, pp. 960-976.

Scheffner, M., Nuber, U., and Huibregtse, J.M. (1995). Protein ubiquitination involving an E1-E2E3 enzyme ubiquitin thioester cascade. In Nature (Nature Publishing Group), pp. 81-83.

Schneider, C.A., Rasband, W.S., and Eliceiri, K.W. (2012). NIH Image to ImageJ: 25 years of image analysis. In Nat Methods (Nature Publishing Group), pp. 671-675.

Schulman, B.A., and Harper, J.W. (2009). Ubiquitin-like protein activation by E1 enzymes: the apex for downstream signalling pathways. In Nature Reviews Molecular Cell Biology (Nature Publishing Group), pp. 319-331.

Seaman, M.N.J. (2004). Cargo-selective endosomal sorting for retrieval to the Golgi requires retromer. In The Journal of cell biology (Rockefeller University Press), pp. 111-122.

Seaman, M.N.J. (2012). The retromer complex - endosomal protein recycling and beyond. In J Cell Sci (The Company of Biologists Ltd), pp. 4693-4702.

Seaman, M.N.J. (2021). The Retromer Complex: From Genesis to Revelations. Trends in biochemical sciences.

Seaman, M.N.J., Harbour, M.E., Tattersall, D., Read, E., and Bright, N. (2009). Membrane recruitment of the cargo-selective retromer subcomplex is catalysed by the small GTPase Rab7 and inhibited by the Rab-GAP TBC1D5. In J Cell Sci (The Company of Biologists Ltd), pp. 23712382.

Settembre, C., Di Malta, C., Polito, V.A., Garcia Arencibia, M., Vetrini, F., Erdin, S., Erdin, S.U., Huynh, T., Medina, D., Colella, P., et al. (2011). TFEB links autophagy to lysosomal biogenesis. In Science (American Association for the Advancement of Science), pp. 1429-1433.

Shang, L., Chen, S., Du, F., Li, S., Zhao, L., and Wang, X. (2011). Nutrient starvation elicits an acute autophagic response mediated by Ulk1 dephosphorylation and its subsequent dissociation from AMPK. In Proc Natl Acad Sci USA (National Academy of Sciences), pp. 47884793.

Shen, K., and Sabatini, D.M. (2018). Ragulator and SLC38A9 activate the Rag GTPases through noncanonical GEF mechanisms. In Proc Natl Acad Sci USA (National Academy of Sciences), pp. 9545-9550.

Shinde, S.R., and Maddika, S. (2016). PTEN modulates EGFR late endocytic trafficking and degradation by dephosphorylating Rab7. In Nat Commun (Nature Publishing Group), pp. 10689-10611. 
Singh, J., Kaade, E., Muntel, J., Bruderer, R., Reiter, L., Thelen, M., and Winter, D. (2020). Systematic Comparison of Strategies for the Enrichment of Lysosomes by Data Independent Acquisition. Journal of Proteome Research 19, 371-381.

Soleimani Zakeri, N.S., Pashazadeh, S., and MotieGhader, H. (2020). Gene biomarker discovery at different stages of Alzheimer using gene co-expression network approach. In Sci Rep (Nature Publishing Group), pp. 12210-12213.

Song, P., Trajkovic, K., Tsunemi, T., and Krainc, D. (2016). Parkin Modulates Endosomal Organization and Function of the Endo-Lysosomal Pathway. In $\mathrm{J}$ Neurosci (Society for Neuroscience), pp. 2425-2437.

Sowa, M.E., Bennett, E.J., Gygi, S.P., and Harper, J.W. (2009). Defining the human deubiquitinating enzyme interaction landscape. Cell 138, 389-403.

Spinosa, M.R., Progida, C., De Luca, A., Colucci, A.M.R., Alifano, P., and Bucci, C. (2008). Functional characterization of Rab7 mutant proteins associated with Charcot-Marie-Tooth type 2B disease. In J Neurosci (Society for Neuroscience), pp. 1640-1648.

Stroupe, C. (2018). This Is the End: Regulation of Rab7 Nucleotide Binding in Endolysosomal Trafficking and Autophagy. In Front Cell Dev Biol (Frontiers), pp. 129.

Sun, J., Liu, Y., Jia, Y., Hao, X., Lin, W.J., Tran, J., Lynch, G., Baudry, M., and Bi, X. (2018). UBE3Amediated p18/LAMTOR1 ubiquitination and degradation regulate mTORC1 activity and synaptic plasticity. In Elife (eLife Sciences Publications Limited), pp. 1196.

Takahashi, S., Kubo, K., Waguri, S., Yabashi, A., Shin, H.-W., Katoh, Y., and Nakayama, K. (2012). Rab11 regulates exocytosis of recycling vesicles at the plasma membrane. In J Cell Sci, pp. 4049-4057.

Thomas, G.M., Hayashi, T., Chiu, S.-L., Chen, C.-M., and Huganir, R.L. (2012). Palmitoylation by DHHC5/8 targets GRIP1 to dendritic endosomes to regulate AMPA-R trafficking. In Neuron, pp. 482-496.

Tsun, Z.-Y., Bar-Peled, L., Chantranupong, L., Zoncu, R., Wang, T., Kim, C., Spooner, E., and Sabatini, D.M. (2013). The folliculin tumor suppressor is a GAP for the RagC/D GTPases that signal amino acid levels to mTORC1. In Mol Cell, pp. 495-505.

Tyanova, S., Temu, T., and Cox, J. (2016a). The MaxQuant computational platform for mass spectrometry-based shotgun proteomics. In Nat Protoc (Nature Publishing Group), pp. 23012319.

Tyanova, S., Temu, T., Sinitcyn, P., Carlson, A., Hein, M.Y., Geiger, T., Mann, M., and Cox, J. (2016b). The Perseus computational platform for comprehensive analysis of (prote)omics data. In Nat Methods (Nature Publishing Group), pp. 731-740.

Ullrich, O., Horiuchi, H., Bucci, C., and Zerial, M. (1994). Membrane association of Rab5 mediated by GDP-dissociation inhibitor and accompanied by GDP/GTP exchange. In Nature (Nature Publishing Group), pp. 157-160.

Ullrich, O., Reinsch, S., Urbé, S., Zerial, M., and Parton, R.G. (1996). Rab11 regulates recycling through the pericentriolar recycling endosome. In The Journal of cell biology, pp. 913-924.

van der Kant, R., Fish, A., Janssen, L., Janssen, H., Krom, S., Ho, N., Brummelkamp, T., Carette, J., Rocha, N., and Neefjes, J. (2013). Late endosomal transport and tethering are coupled processes controlled by RILP and the cholesterol sensor ORP1L. In J Cell Sci (The Company of Biologists Ltd), pp. 3462-3474.

van der Sluijs, P., Hull, M., Webster, P., Mâle, P., Goud, B., and Mellman, I. (1992). The small GTPbinding protein rab4 controls an early sorting event on the endocytic pathway. In Cell, pp. 729740.

van Wijk, S.J.L., and Timmers, H.T.M. (2010). The family of ubiquitin-conjugating enzymes (E2s): deciding between life and death of proteins. In FASEB J (John Wiley \& Sons, Ltd), pp. 981-993.

Vicinanza, M., Dapos, A., Giovanni, Di Campli, A., and De Matteis, M.A. (2008). Function and dysfunction of the PI system in membrane trafficking. In EMBO J (John Wiley \& Sons, Ltd), pp. 2457-2470. 
Wagner, S.A., Beli, P., Weinert, B.T., Nielsen, M.L., Cox, J., Mann, M., and Choudhary, C. (2011). A proteome-wide, quantitative survey of in vivo ubiquitylation sites reveals widespread regulatory roles. In Mol Cell Proteomics, pp. M111.013284.

Wan, W., You, Z., Zhou, L., Xu, Y., Peng, C., Zhou, T., Yi, C., Shi, Y., and Liu, W. (2018). mTORC1Regulated and HUWE1-Mediated WIPI2 Degradation Controls Autophagy Flux. In Mol Cell, pp. 303-315.e306.

Wang, S., and Bellen, H.J. (2015). The retromer complex in development and disease. Development (Cambridge, England) 142, 2392-2396.

Wilcke, M., Johannes, L., Galli, T., Mayau, V., Goud, B., and Salamero, J. (2000). Rab11 regulates the compartmentalization of early endosomes required for efficient transport from early endosomes to the trans-golgi network. In The Journal of cell biology, pp. 1207-1220.

Williams, E.T., Chen, X., and Moore, D.J. (2017). VPS35, the Retromer Complex and Parkinson\&apos;s Disease. In J Parkinsons Dis (IOS Press), pp. 219-233.

Wu, M., Wang, T., Loh, E., Hong, W., and Song, H. (2005). Structural basis for recruitment of RILP by small GTPase Rab7. In EMBO J (John Wiley \& Sons, Ltd), pp. 1491-1501.

Wu, Y.-W., Goody, R.S., Abagyan, R., and Alexandrov, K. (2009). Structure of the disordered C terminus of Rab7 GTPase induced by binding to the Rab geranylgeranyl transferase catalytic complex reveals the mechanism of Rab prenylation. In J Biol Chem, pp. 13185-13192.

Yau, R., and Rape, M. (2016). The increasing complexity of the ubiquitin code. In Nat Cell Biol (Nature Publishing Group), pp. 579-586.

Yim, W.W.-Y., and Mizushima, N. (2020). Lysosome biology in autophagy. In Cell Discov (Nature Publishing Group), pp. 6-12.

Yonehara, R., Nada, S., Nakai, T., Nakai, M., Kitamura, A., Ogawa, A., Nakatsumi, H., Nakayama, K.I., Li, S., Standley, D.M., et al. (2017). Structural basis for the assembly of the Ragulator-Rag GTPase complex. In Nat Commun (Nature Publishing Group), pp. 1625-1611.

Yoshida, Y., Yasuda, S., Fujita, T., Hamasaki, M., Murakami, A., Kawawaki, J., Iwai, K., Saeki, Y., Yoshimori, T., Matsuda, N., et al. (2017). Ubiquitination of exposed glycoproteins by SCFFBXO27 directs damaged lysosomes for autophagy. In Proc Natl Acad Sci USA (National Academy of Sciences), pp. 8574-8579.

Yu, L., McPhee, C.K., Zheng, L., Mardones, G.A., Rong, Y., Peng, J., Mi, N., Zhao, Y., Liu, Z., Wan, F., et al. (2010). Termination of autophagy and reformation of lysosomes regulated by mTOR. In Nature (Nature Publishing Group), pp. 942-946.

Zheng, N., and Shabek, N. (2017). Ubiquitin Ligases: Structure, Function, and Regulation. In Annu Rev Biochem ( Annual Reviews), pp. 129-157.

Zoncu, R., Bar-Peled, L., Efeyan, A., Wang, S., Sancak, Y., and Sabatini, D.M. (2011). mTORC1 senses lysosomal amino acids through an inside-out mechanism that requires the vacuolar $\mathrm{H}(+)$-ATPase. In Science (American Association for the Advancement of Science), pp. 678-683. 


\section{Acknowledgments}

First and foremost, I would like to express my deepest gratitude to my supervisor Dr. Anja Bremm. Thank you for your continuous support and motivation! I was your first master student and will end the first generation of PhD students in the lab. During these years, you showed me how to shape ideas, tackle problems, and simply do research. This long journey shaped me both as person and scientist. I'm proud that we together managed to finish this project successfully, with three children and a pandemic.

My thanks go also to Prof. Dr. Martin Grininger who agreed to be my official supervisor, as well as to the other members of the thesis committee.

I want to acknowledge my collaboration partners for their contributions to this project: special thanks to the "mass spec guys" Florian Bonn and Georg Tascher. Florian Steinberg supported us with data for the Rab7 part. This work wouldn't have been possible without their expertise.

During the time of my Master's thesis in 2015, I was glad to attend the last IBCII retreat in Neckarsulm where I got a note with a quote from Theodor Fontane: "Das Beste aber, dem du begegnen wirst, werden die Menschen sein". This note staid with me the entire six years since it is just true. I'm deeply grateful for the people who I met.

Without my beloved colleagues Julia, Mila, and Verena, PhD life wouldn't have been the same. We did not just share lab and bench space, but also private time and life. I'm happy that I know you, even though our paths diverged.

Special thanks here to all members of IBCII for the discussions, advises, and support, physically with material and mentally with fun times. I enjoy every moment working in this scientific and collegial community. In this manner, I want to appreciate the extra support of Marcel and Jan for proofreading parts of this thesis.

The last years were the most exciting, life-changing, and challenging time in my life. Last but most important, I want to thank my family:

Liebe "Muddä", lieber "Vaddä", ich kann euch nicht genug danken, dass ihr immer für mich da seid und mich unterstützt, wenn auch nicht immer vor Ort, aber immer in meinem Herzen. Ihr seid die besten und wahren Helden für mich!

Lieber Tobi, du hast wahrscheinlich den Grundstein für meine naturwissenschaftliche Karriere gelegt, nachdem ich als kleine Schwester dir auch in der Wahl des Bio LKs nacheifern wollte. Bester großer Bruder, danke dir!

Liebster Hendrik, nach 15 gemeinsamen Jahren gibt es gar nicht genug Worte, um dir zu danken. Du bist immer für mich da und machst mich zu dem Menschen, der ich bin. Mit dir gehe ich den wohl schönsten, aufregendsten und anstrengendsten Weg des Lebens als Eltern. Franz, die Zukunft gehört dir! 


\section{VIII.Curriculum Vitae}

Alexandra Hertel (née Kalb)

Adress:

Phone:

Email:

Born:

\section{Education:}

Since Mar./2016

Oct./2013 - Dec./ 2015

Oct./2009 - Sept./2013

Aug./2000 - June/2009

Professional Experience:

Since Jan./2016
Seebachstraße 39, 65929 Frankfurt +491776540900

alexandrakalb.nbg@gmail.com, 24/September/1989 in Nürnberg

GOETHE UNIVERSITY, Frankfurt/Main

Biochemistry, PhD

Degree expected in 2021: Dr. phil. nat.

GOETHE UNIVERSITY, Frankfurt/Main

Biochemistry, master degree course

Master of Science, grade: 1.8 (good)

TECHNISCHE HOCHSCHULE GEORG SIMON OHM, Nürnberg Applied Chemistry (specialization Biochemistry), bachelor degree course Bachelor of Science, grade: 1.6 (good)

NEUES GYMNASIUM, Nürnberg

Abitur (general qualification for university entrance), grade: 1.7 (good)

\footnotetext{
Apr./2015 - Dec./2015

Jan./2015 - Feb./2015

Sept./2014 - Dec./2014

Oct./2012 - Aug./2013

Mar./2011 - Aug./2011

BUCHMANN INSTITUTE FOR MOLECULAR LIFE SCIENCES,

Frankfurt/Main

Master's thesis, Group Anja Bremm

"Characterizing the role of USP32 in retromer-mediated endosomal protein sorting and its impact on autophagosome formation"

GEORG-SPEYER-HAUS, Frankfurt/Main

Internship, Group Martin Zörnig

BUCHMANN INSTITUTE FOR MOLECULAR LIFE SCIENCES,

Frankfurt/Main

Internship/student assistant, Group Anja Bremm

ABBVIE DEUTSCHLAND GMBH \& CO. KG, Ludwigshafen

Bachelor's thesis/Internship, Pre \& Early NBE Development Sciences,

„Entwicklung eines High-Throughput-Screenings für feste und flüssige Antikörper-Formulierungen in 96 well Mikrotiterplatten"

SCHWAN-STABILO COSMETICS GMBH \& Co. KG, Heroldsberg

Internship, Process Development
} 
Associations:

Since Sept./2017

Research Unit FOR2625 “Mechanisms of Lysosomal Homeostasis"

Since Aug./2016

Goethe Research Academy for Early Career Researchers (GRADE)

Since Jan./2016

Integrative Research Graduate Program (IRTG) of SFB1177 on Autophagy

\section{Publications:}

Mila Basic, Alexandra Hertel, Justyna Cichon, Florian Bonn, Mariana Tellechea, Alexandra Stolz, Andreas Kern, Christian Behl, and Anja Bremm

"The deubiquitinase USP11 is a versatile and conserved regulator of autophagy"

Manuscript under revision in Journal of Biological Chemistry (JBC/2021/018306)

Alexandra Hertel, Florian Bonn, Georg Tascher, Ivan Dikic, Florian Steinberg, Anja Bremm "USP32 regulates non-proteolytic LAMTOR1 ubiquitination and Ragulator function" Manuscript in preparation

\section{Contributions at conferences:}

Talk at CSHL UBIQUITINS, AUTOPHAGY, \& DISEASES 2021 VIRTUAL MEETING "USP32 regulates non-proteolytic LAMTOR1 ubiquitination and Ragulator function", Alexandra Hertel, Florian Bonn, Georg Tascher, Ivan Dikic, Florian Steinberg, Anja Bremm Poster at 2nd Frankfurt Conference on Quality Control in Life Processes 2018, Frankfurt/Main "The deubiquitylase USP32 is a novel player in the endosomal-lysosomal system",

Alexandra Kalb, Florian Bonn, Ivan Dikic, Anja Bremm

Poster at EMBO Autophagy Conference 2017, Cavtat, Croatia

"USP11 - a novel regulator of autophagy", Mila Basic, Alexandra Kalb, Anja Bremm

Poster at Frankfurt Conference on Quality Control 2016, Frankfurt/Main

"USP11 - a novel regulator of autophagy", Mila Basic, Alexandra Kalb, Anja Bremm

\section{Language skills:}

German (mother tongue), English (fluent), Spanish (fundamentals), Latin (high qualification) 